\title{
Transportation Routing Analysis Geographic Information System (TRAGIS) User's Manual
}

Revision 0

June 2003

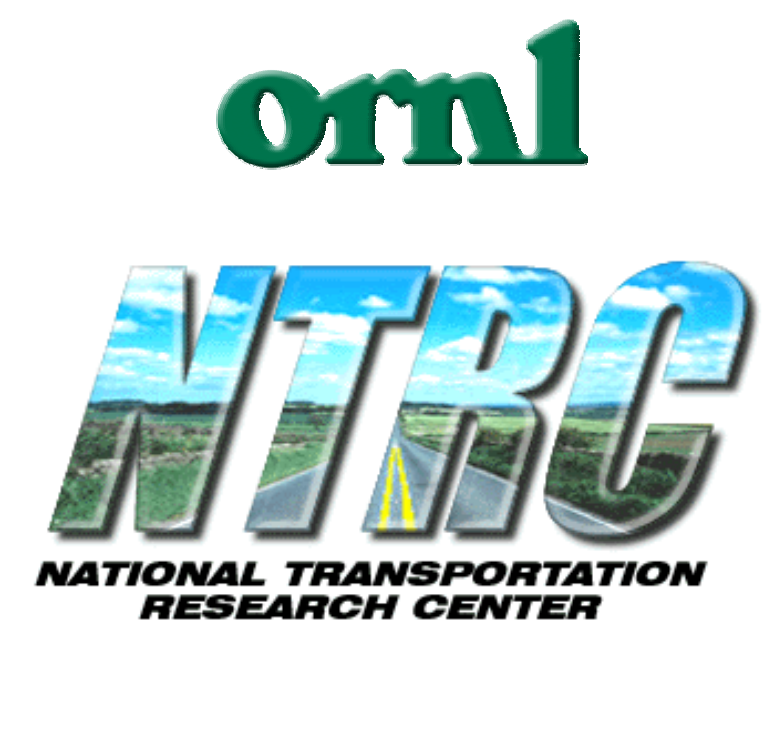




\title{
DOCUMENT AVAILABILITY
}

Reports produced after January 1, 1996, are generally available free via the U.S. Department of Energy (DOE) Information Bridge:

Web site: http://www.osti.gov/bridge

Reports produced before January 1, 1996, may be purchased by members of the public from the following source:

\author{
National Technical Information Service \\ 5285 Port Royal Road \\ Springfield, VA 22161 \\ Telephone: 703-605-6000 (1-800-553-6847) \\ TDD: $703-487-4639$ \\ Fax: 703-605-6900 \\ E-mail: info@ntis.fedworld.gov \\ Web site: http://www.ntis.gov/support/ordernowabout.htm
}

Reports are available to DOE employees, DOE contractors, Energy Technology Data Exchange (ETDE) representatives, and International Nuclear Information System (INIS) representatives from the following source:

Office of Scientific and Technical Information

P.O. Box 62

Oak Ridge, TN 37831

Telephone: 865-576-8401

Fax: 865-576-5728

E-mail: reports@adonis.osti.gov

Web site: http://www.osti.gov/contact.html

This report was prepared as an account of work sponsored by an agency of the United States Government. Neither the United States government nor any agency thereof, nor any of their employees, makes any warranty, express or implied, or assumes any legal liability or responsibility for the accuracy, completeness, or usefulness of any information, apparatus, product, or process disclosed, or represents that its use would not infringe privately owned rights. Reference herein to any specific commercial product, process, or service by trade name, trademark, manufacturer, or otherwise, does not necessarily constitute or imply its endorsement, recommendation, or favoring by the United States Government or any agency thereof. The views and opinions of authors expressed herein do not necessarily state or reflect those of the United States Government or any agency thereof. 


\title{
TRANSPORTATION ROUTING ANALYSIS GEOGRAPHIC INFORMATION SYSTEM (TRAGIS) USER'S MANUAL
}

\author{
P. E. Johnson \\ Computational Sciences and Engineering Division \\ R. D. Michelhaugh \\ Nuclear Science and Technology Division
}

Date Published: June 2003

Prepared for

National Transportation Program

U.S. Department of Energy

Albuquerque, New Mexico 87185

\author{
Prepared by \\ OAK RIDGE NATIONAL LABORATORY \\ P.O. Box 2008 \\ Oak Ridge, Tennessee 37831-6285 \\ managed by \\ UT-Battelle, LLC \\ for the \\ U.S. DEPARTMENT OF ENERGY \\ under contract DE-AC05-00OR22725
}




\section{Revision History}

\begin{tabular}{|c|c|l|}
\hline Revision & Date & Description \\
\hline 0 & $6 / 30 / 2003$ & Initial version. \\
\hline & & \\
\hline & & \\
\hline & & \\
\hline & & \\
\hline & & \\
\hline & & \\
\hline
\end{tabular}




\section{CONTENTS}

$\begin{aligned} \text { Page } & \end{aligned}$

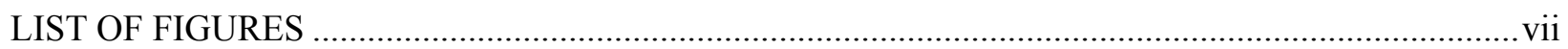

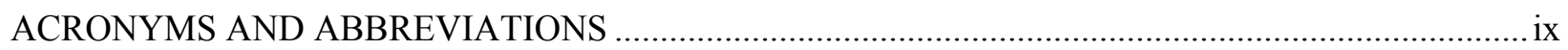

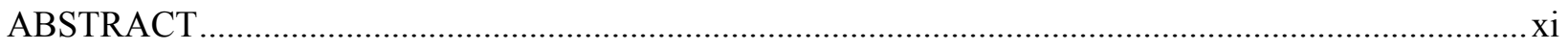

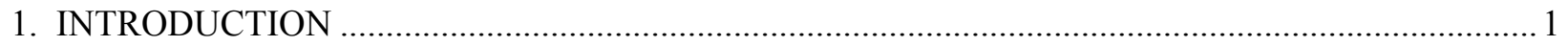

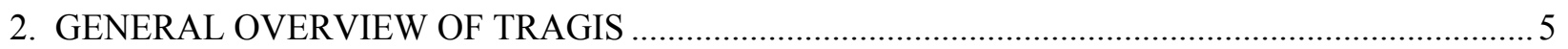

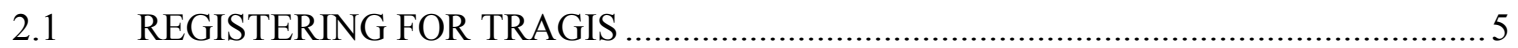

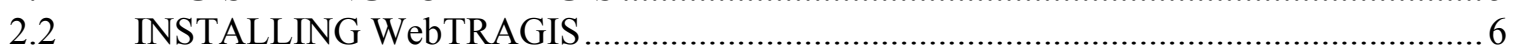

2.3 STARTING WebTRAGIS .................................................................. 9

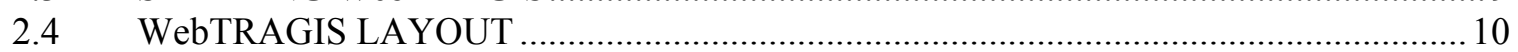

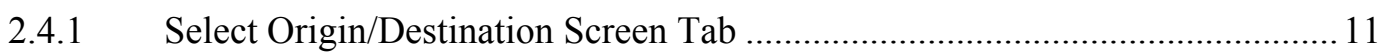

2.4.2 Optional Highway Routing Parameters Screen Tab ....................................... 12

2.4.3 Optional Rail/Water Routing Parameters Screen Tab .................................... 12

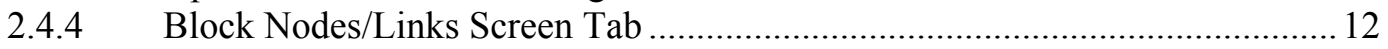

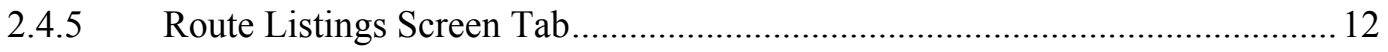

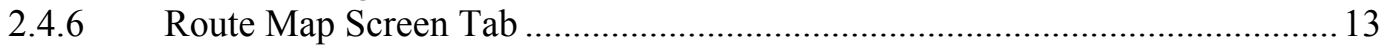

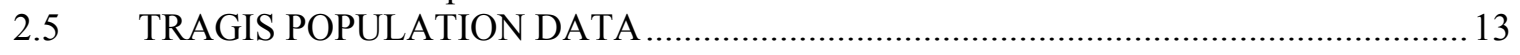

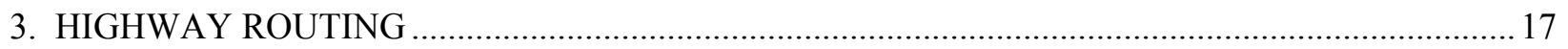

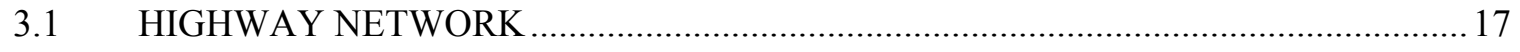

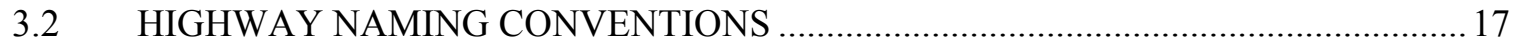

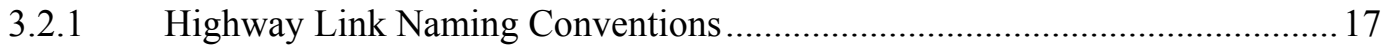

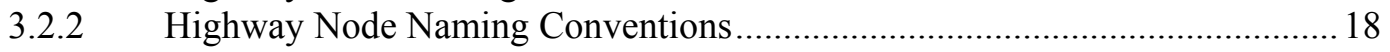

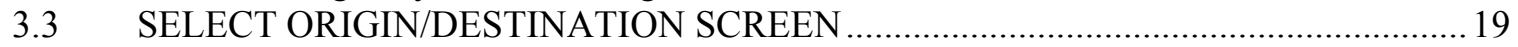

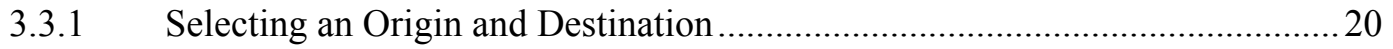

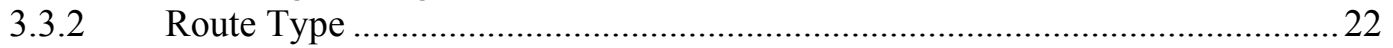

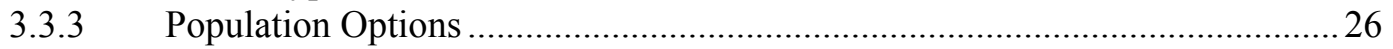

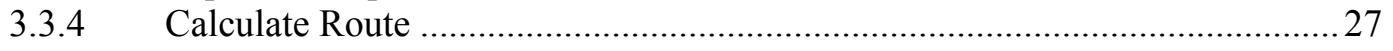

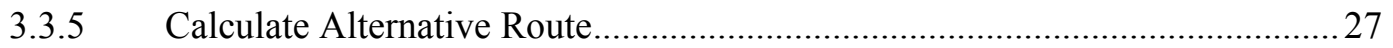

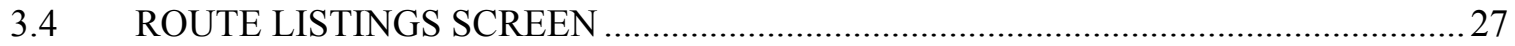

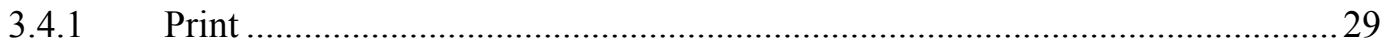

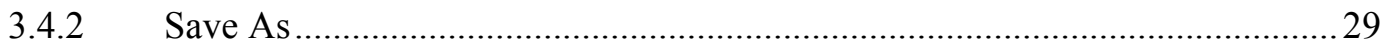

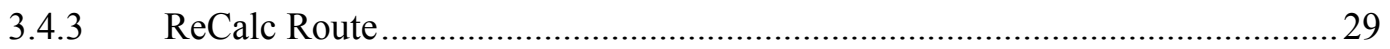

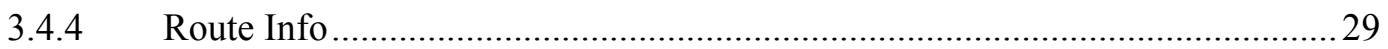

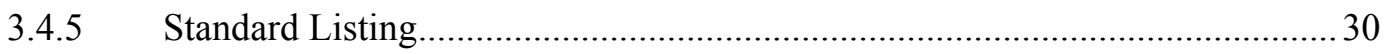

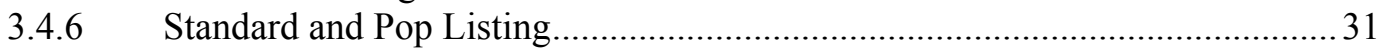

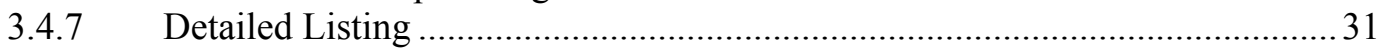

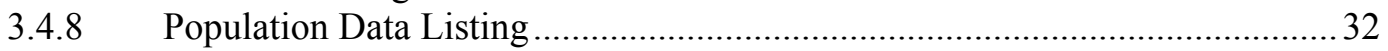

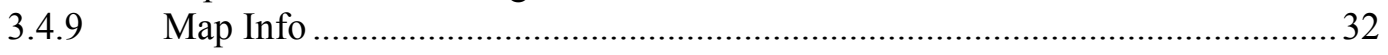

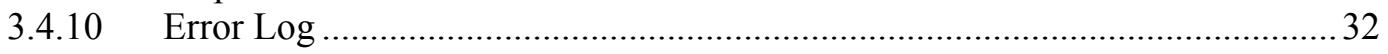

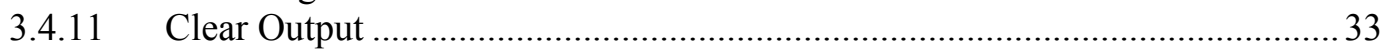

3.5 OPTIONAL HIGHWAY ROUTING PARAMETERS SCREEN …................................ 33 


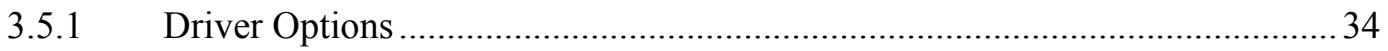

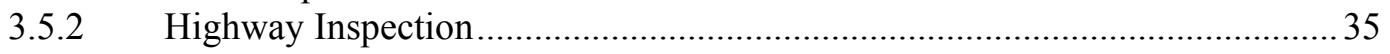

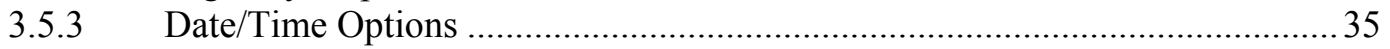

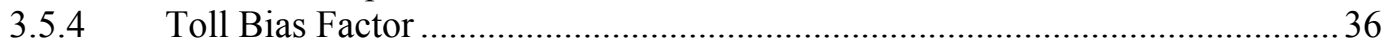

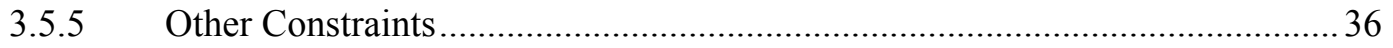

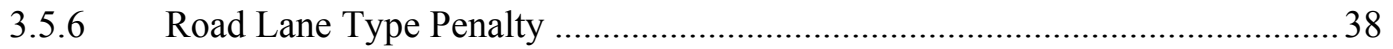

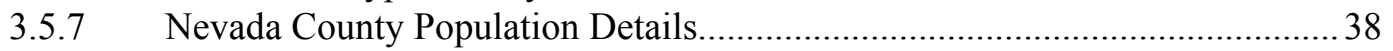

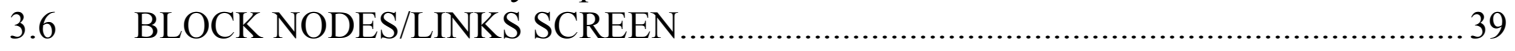

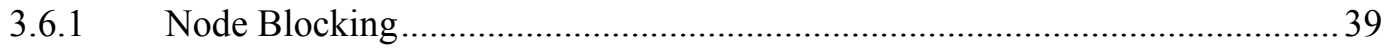

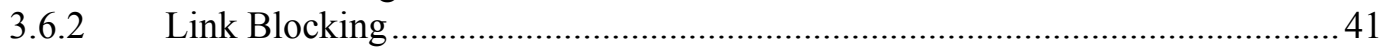

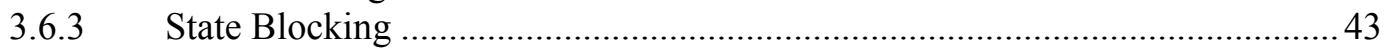

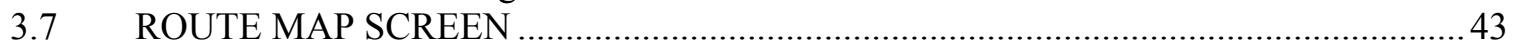

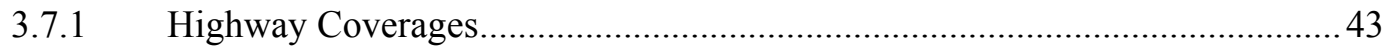

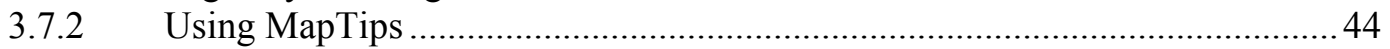

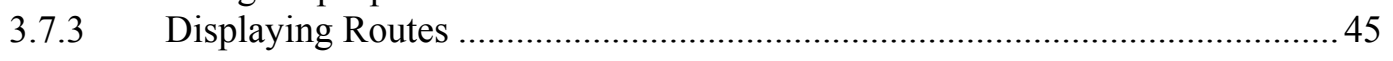

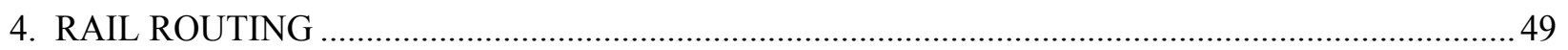

4.1 OPERATIONAL CHARACTERICS OF THE U.S. RAILROAD SYSTEM ..................49

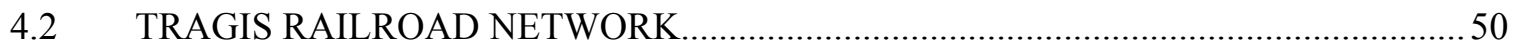

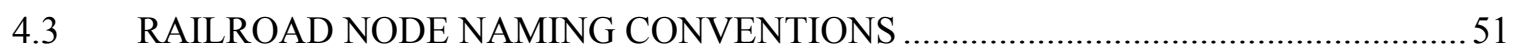

4.4 SELECT ORIGIN/DESTINATION SCREEN ................................................... 52

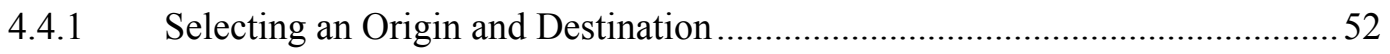

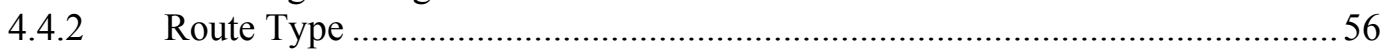

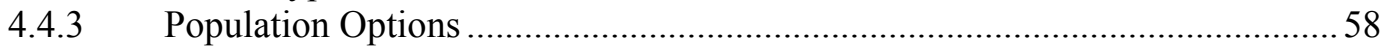

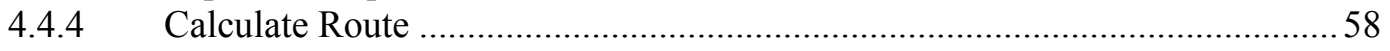

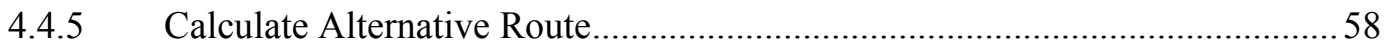

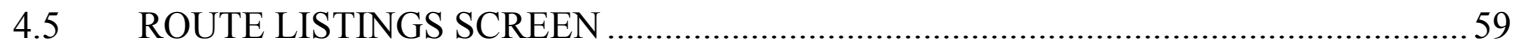

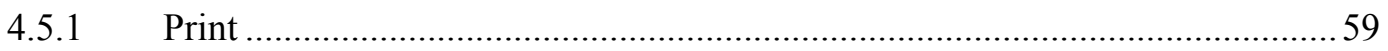

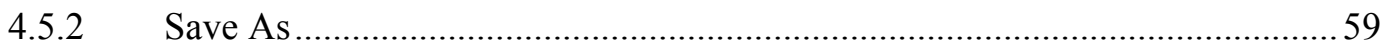

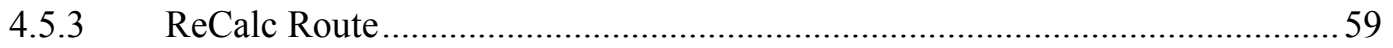

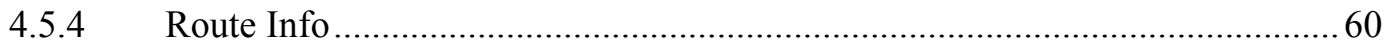

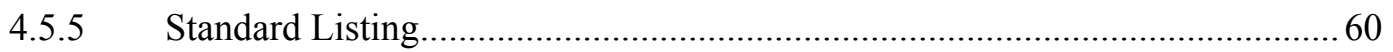

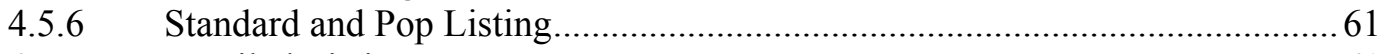

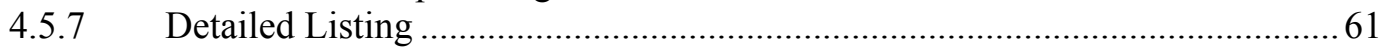

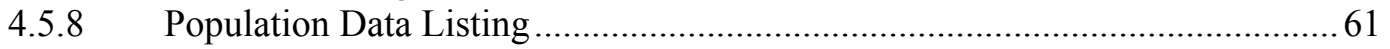

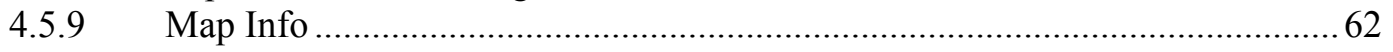

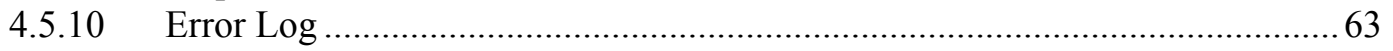

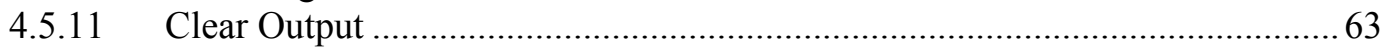

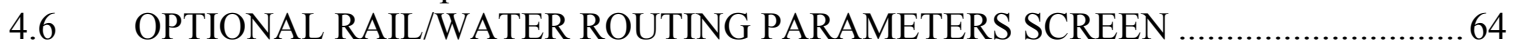

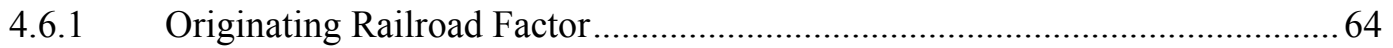

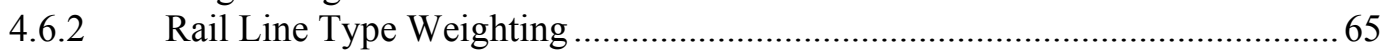

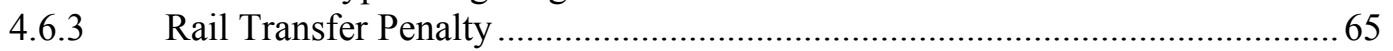

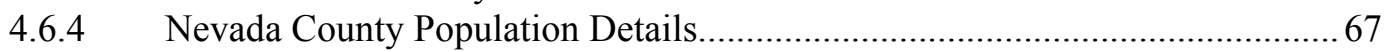

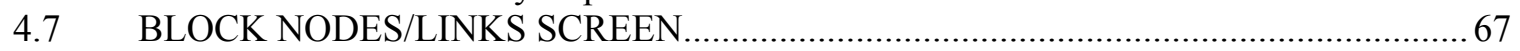

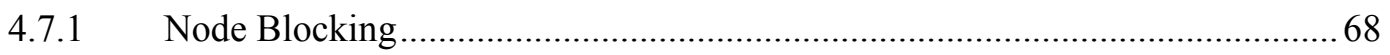

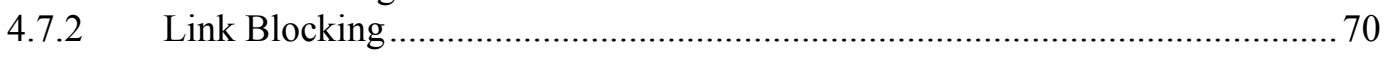

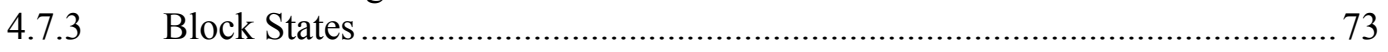

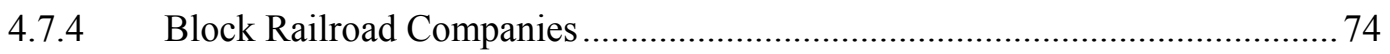

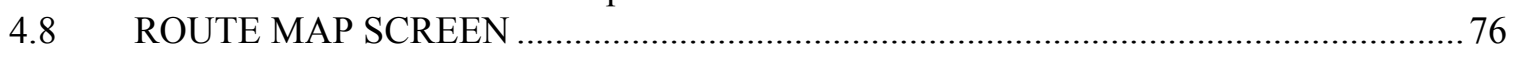

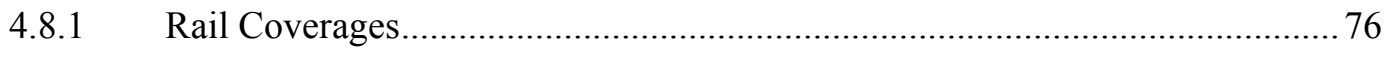




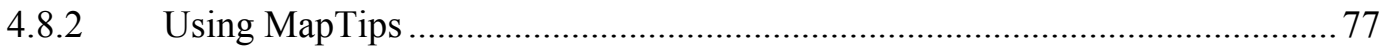

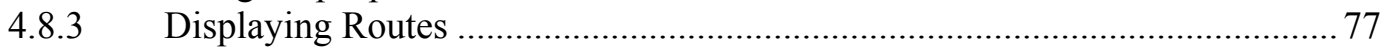

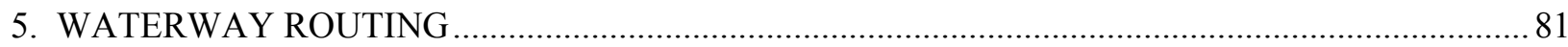

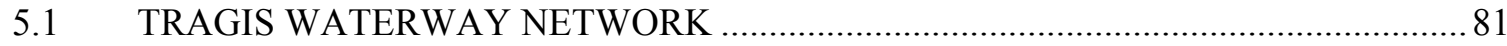

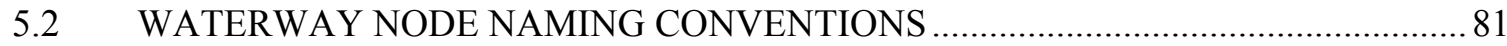

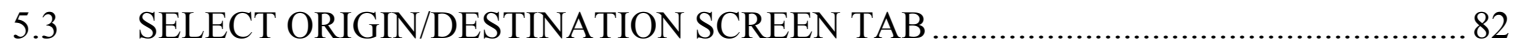

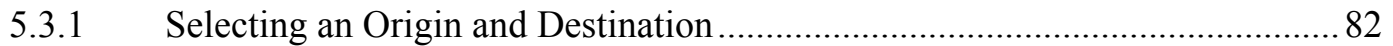

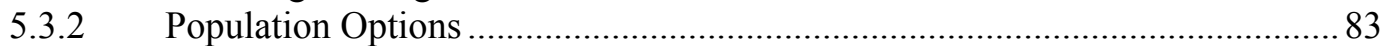

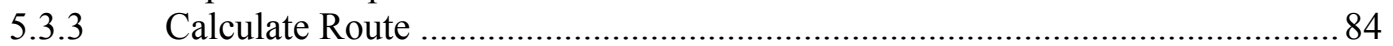

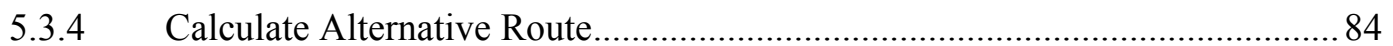

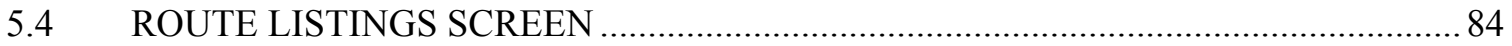

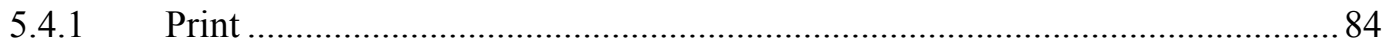

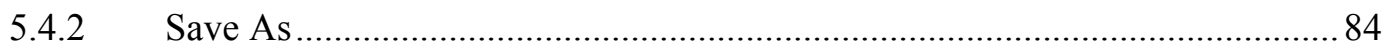

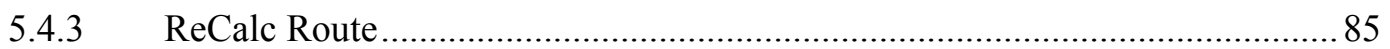

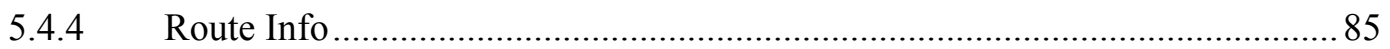

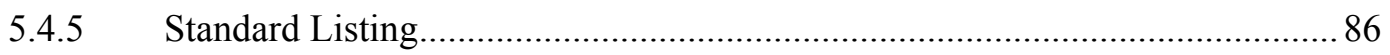

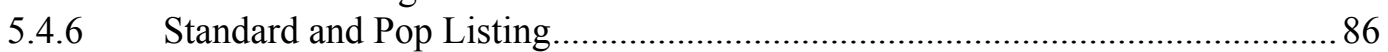

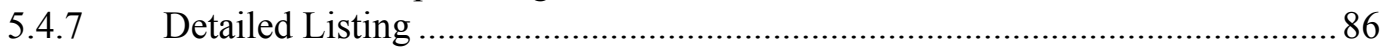

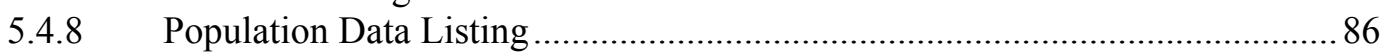

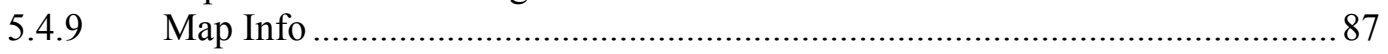

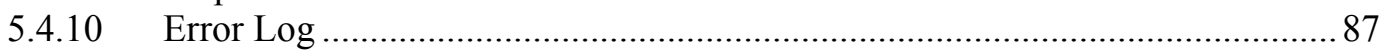

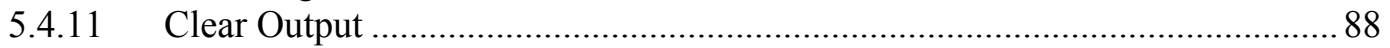

5.5 OPTIONAL RAIL/WATER ROUTING PARAMETERS SCREEN .............................. 88

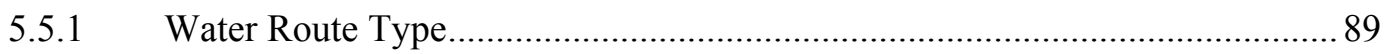

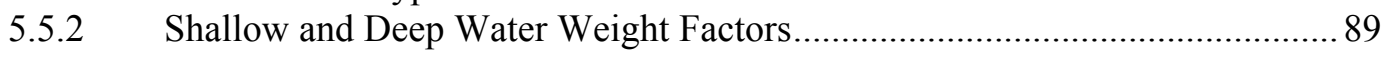

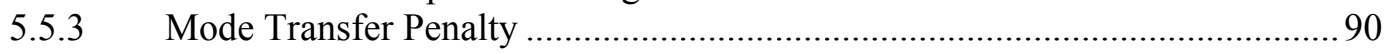

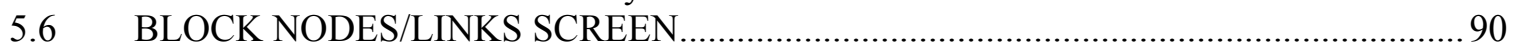

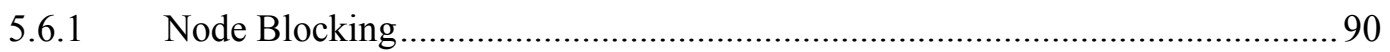

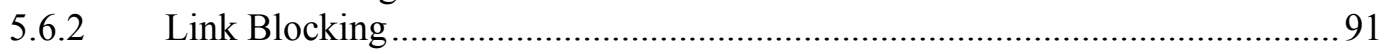

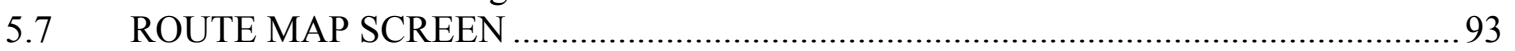

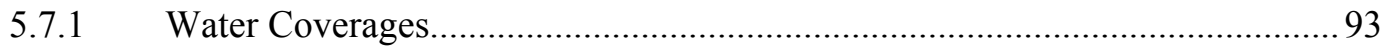

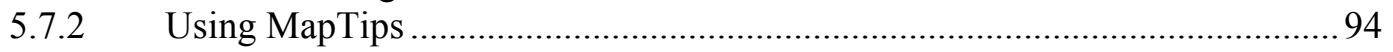

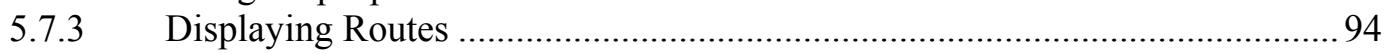

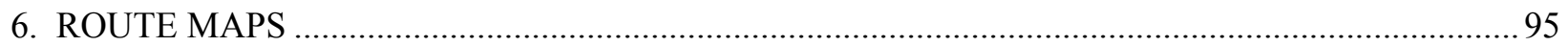

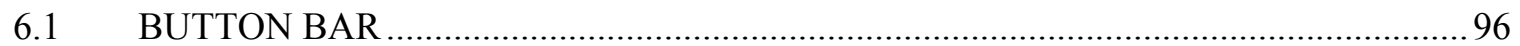

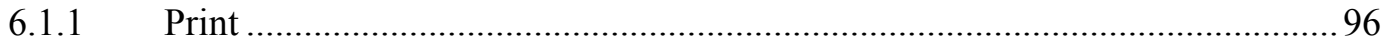

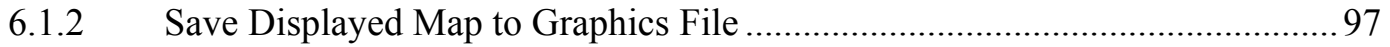

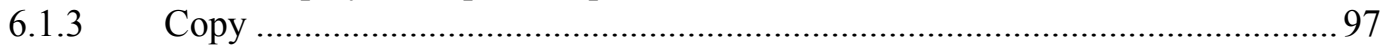

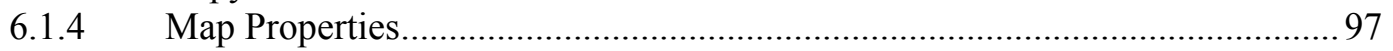

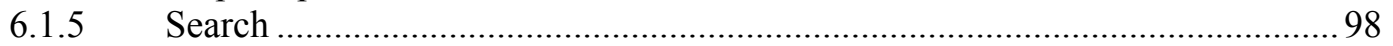

6.1.6 Display Full Extent of Map ..................................................................... 98

6.1.7 Display Map to Extent of Active Layer........................................................... 98

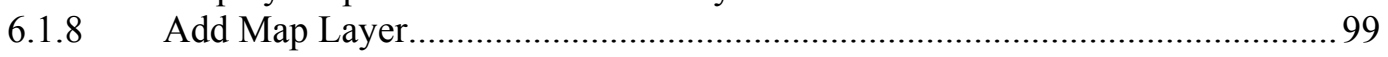

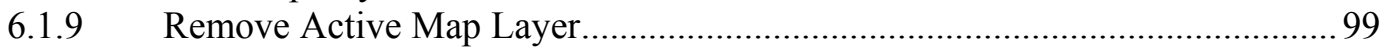

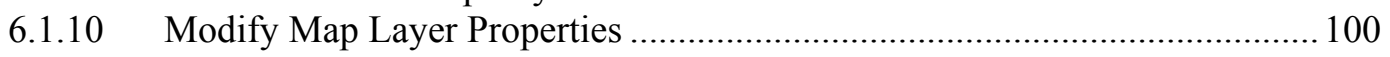

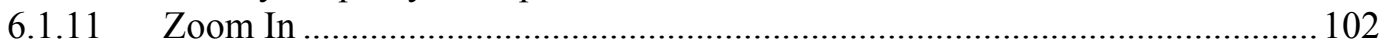

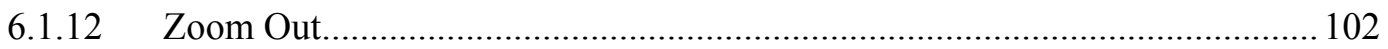

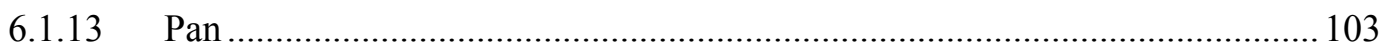




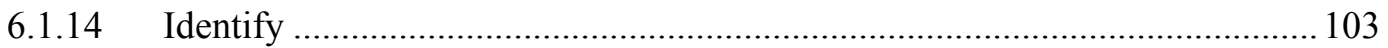

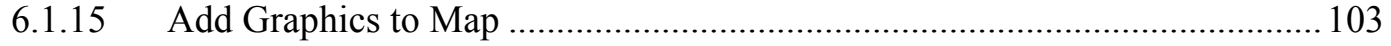

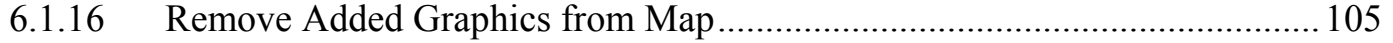

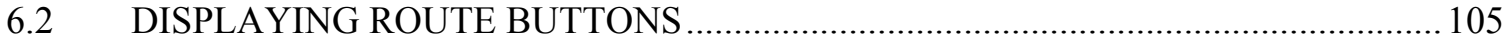

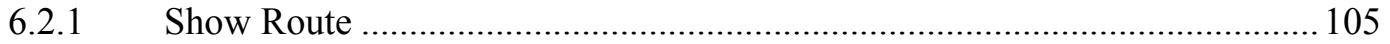

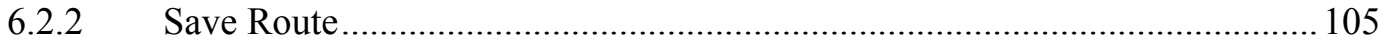

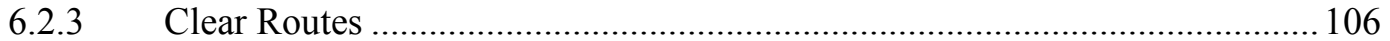

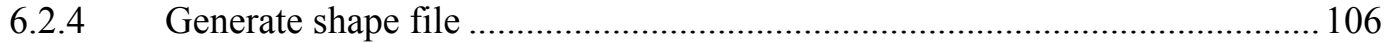

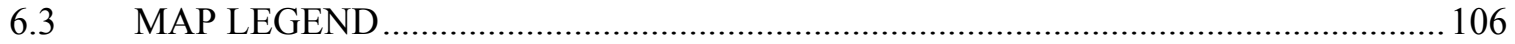

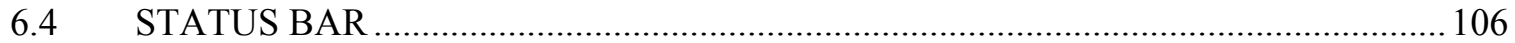

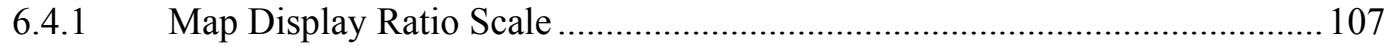

6.4.2 Coordinate Display ................................................................................... 107

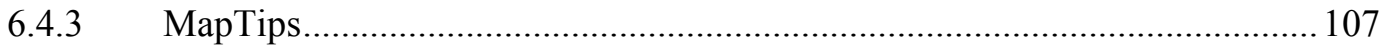

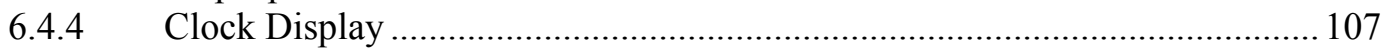

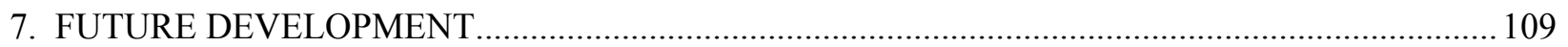

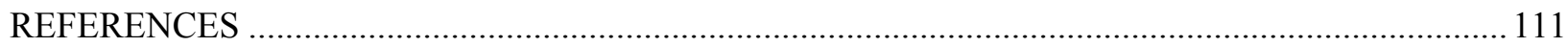

APPENDIX A. SELECTED FACILITY LOCATIONS IN THE TRAGIS HIGHWAY NETWORK...A-3

APPENDIX B. TRAGIS RAILROAD NETWORK ABBREVIATION …............................................ B-3

APPENDIX C. RAIL SHIPMENTS TO YUCCA MOUNTAIN ........................................................

APPENDIX D. SELECTED FACILITY LOCATIONS IN THE TRAGIS RAIL NETWORK ..............D-3 


\section{LIST OF FIGURES}

Figure

Page

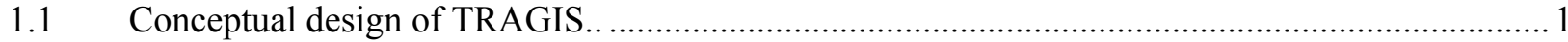

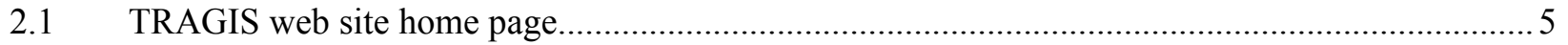

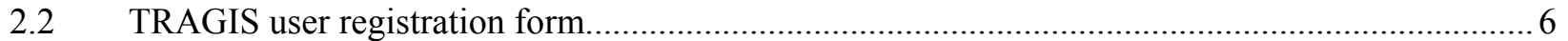

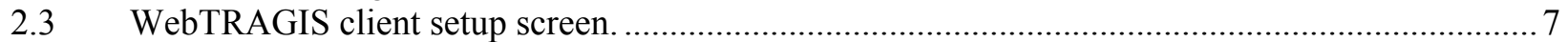

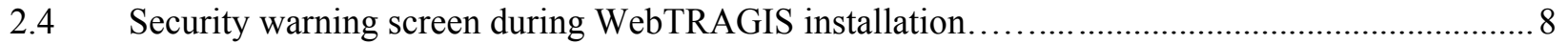

2.5 The InstallShield Wizard for WebTRAGIS Client window. ................................................ 8

2.6 Verification of the installation settings for WebTRAGIS client.............................................. 9

2.7 Verification window indicating successful installation of the WebTRAGIS client. .....................9

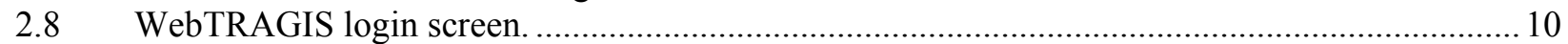

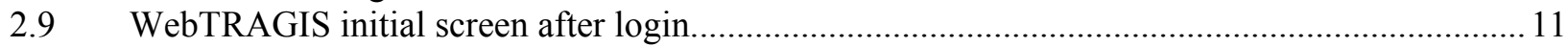

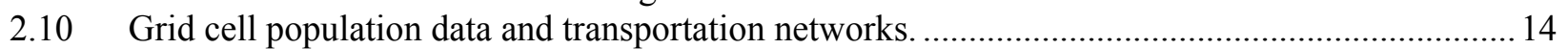

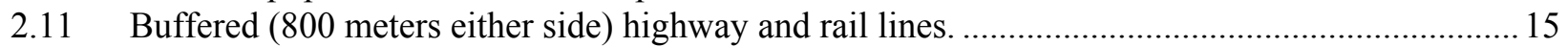

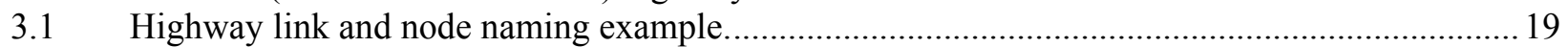

3.2 Select Origin/Destination screen for highway routing.........................................................20

3.3 Example of determining a highway node number from the Route Map screen...........................2 21

3.4 Other route type setting box on highway Select Origin/Destination tab screen........................23

3.5 Nevada Route Options box in the highway Select Origin/Destination tab screen......................25

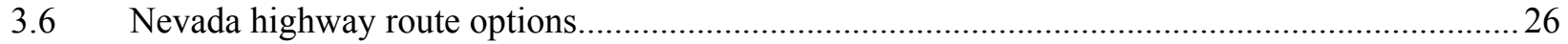

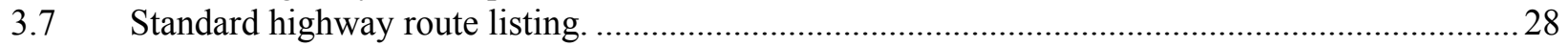

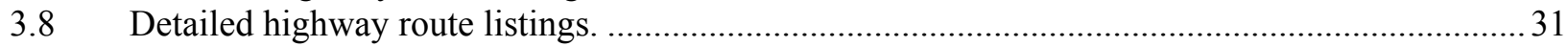

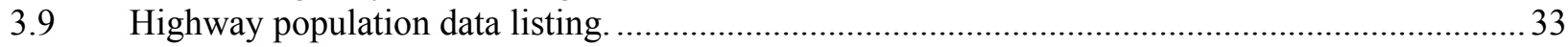

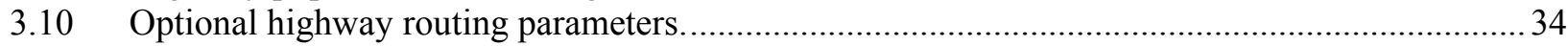

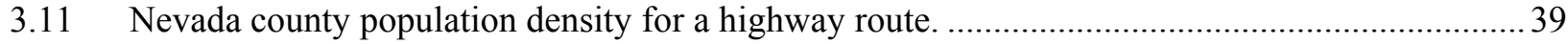

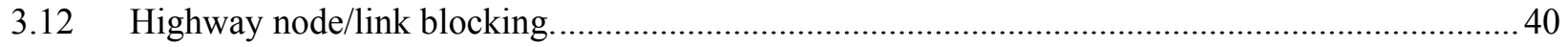

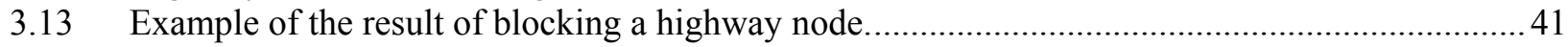

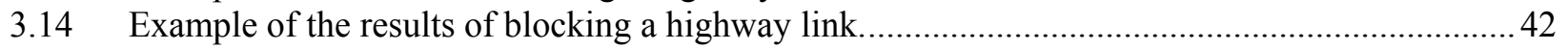

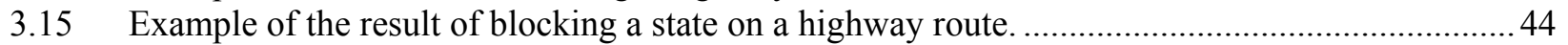

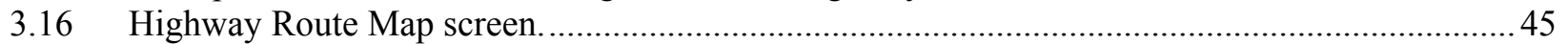

3.17 Difference between the two highway route representations. ..................................................... 46

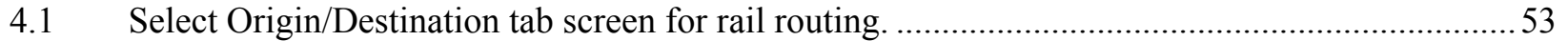

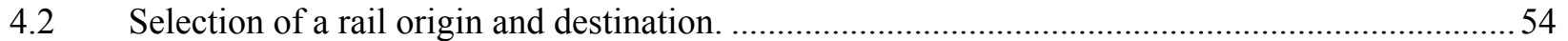

4.3 Example of multiple non unique rail nodes names in Knoxville...............................................55

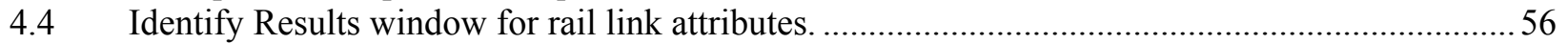

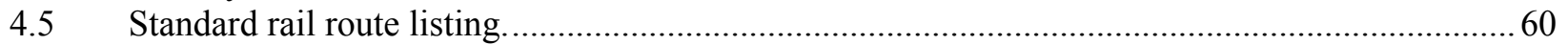

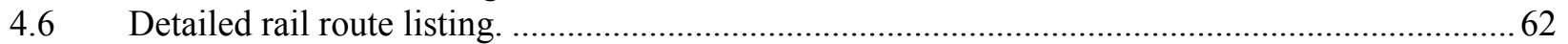

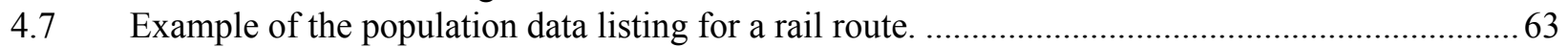

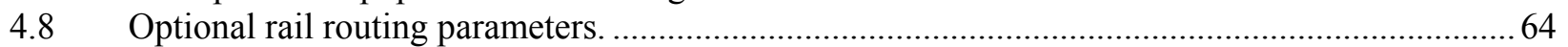

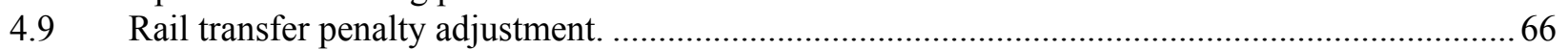

4.10 Example of Nevada county population output for a rail route................................................. 67

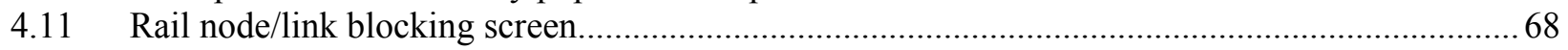

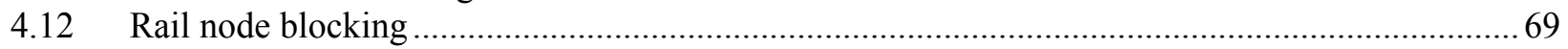

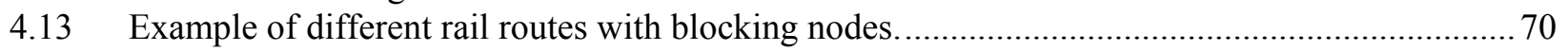

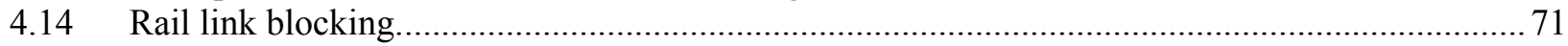

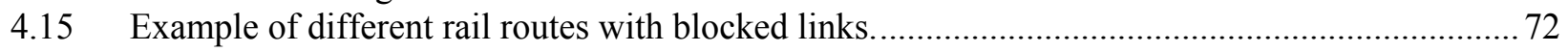

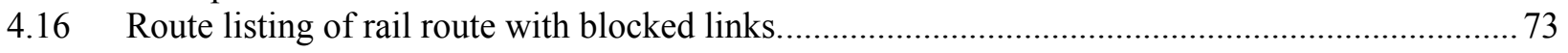




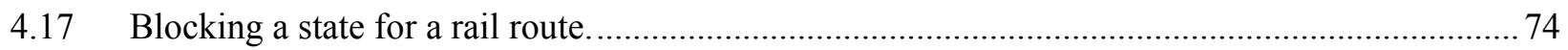

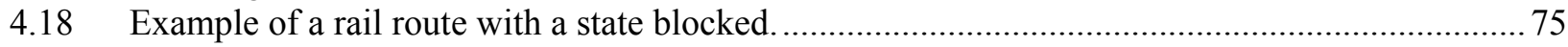

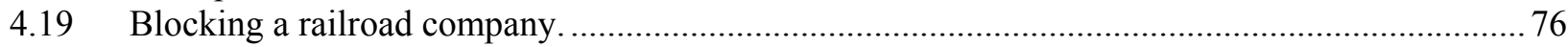

4.20 Example of a rail route with a railroad company blocked. …..................................................... 77

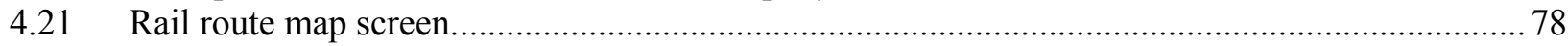

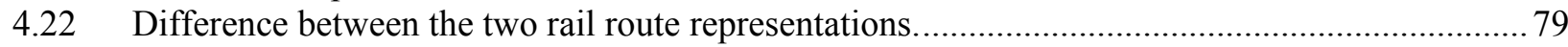

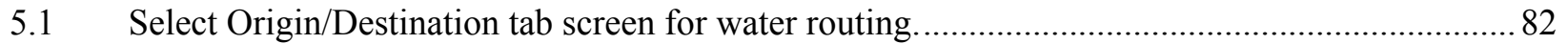

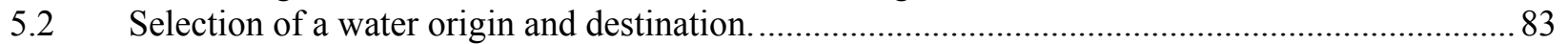

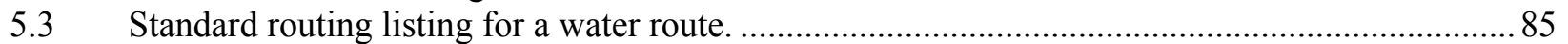

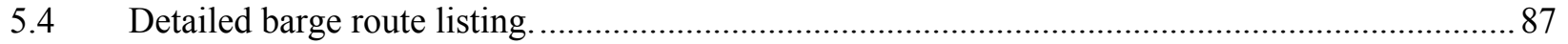

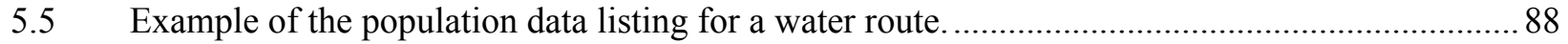

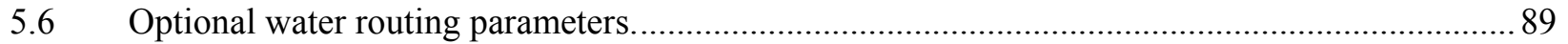

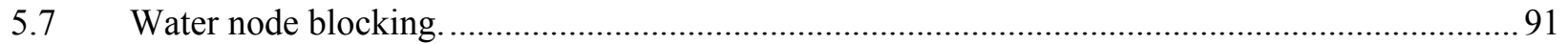

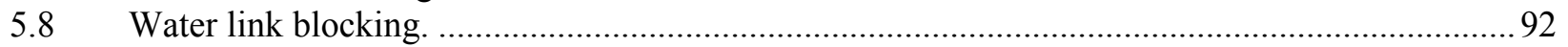

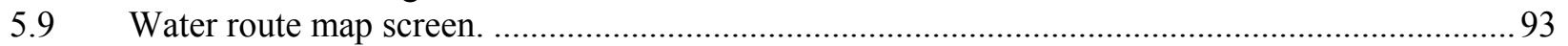

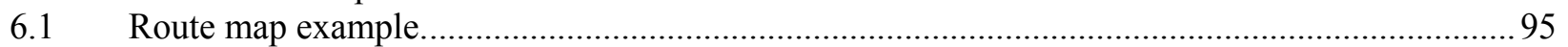

6.2 Print the map screen with the print to fill the page tab active ................................................. 96

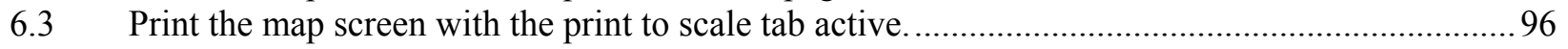

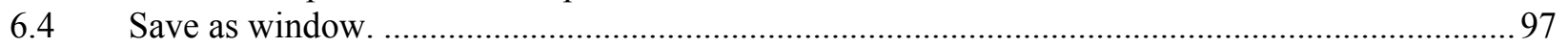

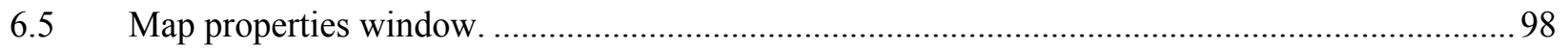

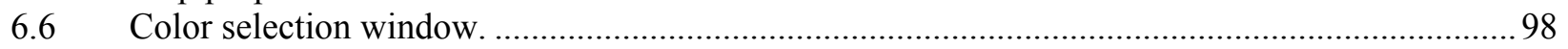

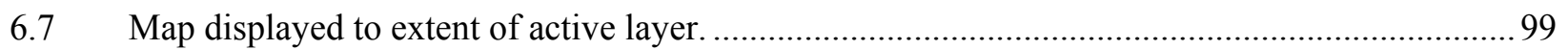

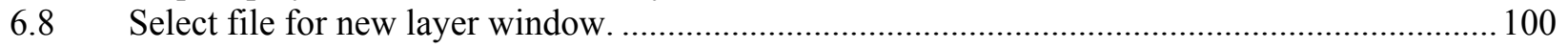

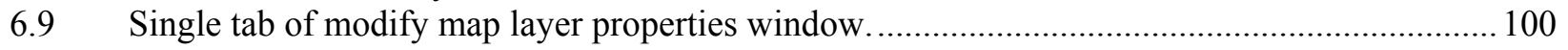

6.10 Unique tab of the modify map layer properties window. ..................................................... 101

6.11 Classes tab of the modify map layer properties window. ................................................ 102

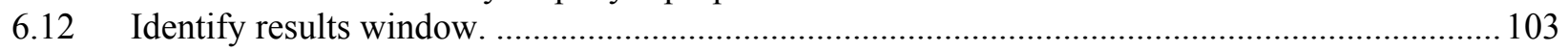

6.13 Additional buttons with add graphics to map button activated............................................. 104

C.1 Proposed rail alignment to the Yucca Mountain Repository....................................................... 


\section{ACRONYMS AND ABBREVIATIONS}

$<\mathrm{C} 3>$

$<\mathrm{OR}>$

$<\mathrm{TR}>$

AMTK

BatchTRAGIS

BNSF

CFR

CMM

$\mathrm{CN}$

$\mathrm{CN}$

CPRS

CSXT

$\mathrm{CZ}$

DOE

DOT

DTED

EM

ESRI

FIPS

GFRR

GIS

GUI

HRCQ

IW

KCS

KXHR

$\mathrm{L} / \mathrm{D}$

MRL

NIMA

NLCD

NP

NS

NTP

ORNL

PC

TIGER

TRAGIS

UP

USG

USGS

WebTRAGIS

WIPP

WWW
Other Class 3 railroads

Other Railroads (non carriers, commuter, museum, tourist, etc.)

Terminal Railroad

Amtrak (National Railroad Passenger Corporation)

specialized client user interface for batch runs of the TRAGIS model

Burlington Northern Santa Fe Railway

Code of Federal Regulations

Commercial Merchant Marine, deep draft waterway

Canada

Canadian National Railway

Canadian Pacific Railway

CSX Transportation

Panama Canal (former Canal Zone FIPS abbreviation)

Department of Energy

Department of Transportation

Digital Terrain Elevation Data

Office of Environmental Restoration and Waste Management

Environmental Systems Research Institute, Inc.

Federal Information Processing Standards

Georgia and Florida Railnet

Geographic Information System

Graphical user interface

Highway Route Controlled Quantity

Inland Waterway, shallow draft waterway

Kansas City Southern Railway

Knoxville and Holston River Railroad

Lock and Dam

Montana Rail Link

National Imagery and Mapping Agency

National Land Cover Database

Nuclear Plant

Norfolk Southern Railway

National Transportation Program

Oak Ridge National Laboratory

Personal computer

Topologically Integrated Geographic Encoding and Referencing system

Transportation Routing Analysis Geographic Information System

Union Pacific Railroad

United States Government

United States Geological Survey

primary client user interface for the TRAGIS model

Waste Isolation Pilot Plant

World Wide Web 
Page intentionally left blank 


\begin{abstract}
The Transportation Routing Analysis Geographic Information System (TRAGIS) model is used to calculate highway, rail, or waterway routes within the United States. TRAGIS is a client-server application with the user interface and map data files residing on the user's personal computer and the routing engine and network data files on a network server. The user's manual provides documentation on installation and the use of the many features of the model.
\end{abstract}


Page intentionally left blank 


\section{INTRODUCTION}

The Transportation Routing Analysis Geographic Information System (TRAGIS) is a user-friendly, geographic information system (GIS)-based transportation routing and analysis computer model. Funding for the development of TRAGIS has been provided by the National Transportation Program (NTP) of the U.S. Department of Energy (DOE). TRAGIS is a client-server application where the user interface and map data files reside on the user's personal computer (PC) and the routing engine and its large data files reside on the server. The model uses the World Wide Web (WWW) for communications between the client and the server. There are two user interfaces for TRAGIS: WebTRAGIS, which is the primary client user interface, and BatchTRAGIS, which is a specialized user interface that allows multiple routes to be prepared and then calculated at one time. Both interfaces rely on the same TRAGIS routing engine and routing networks, as shown on Fig. 1.1.

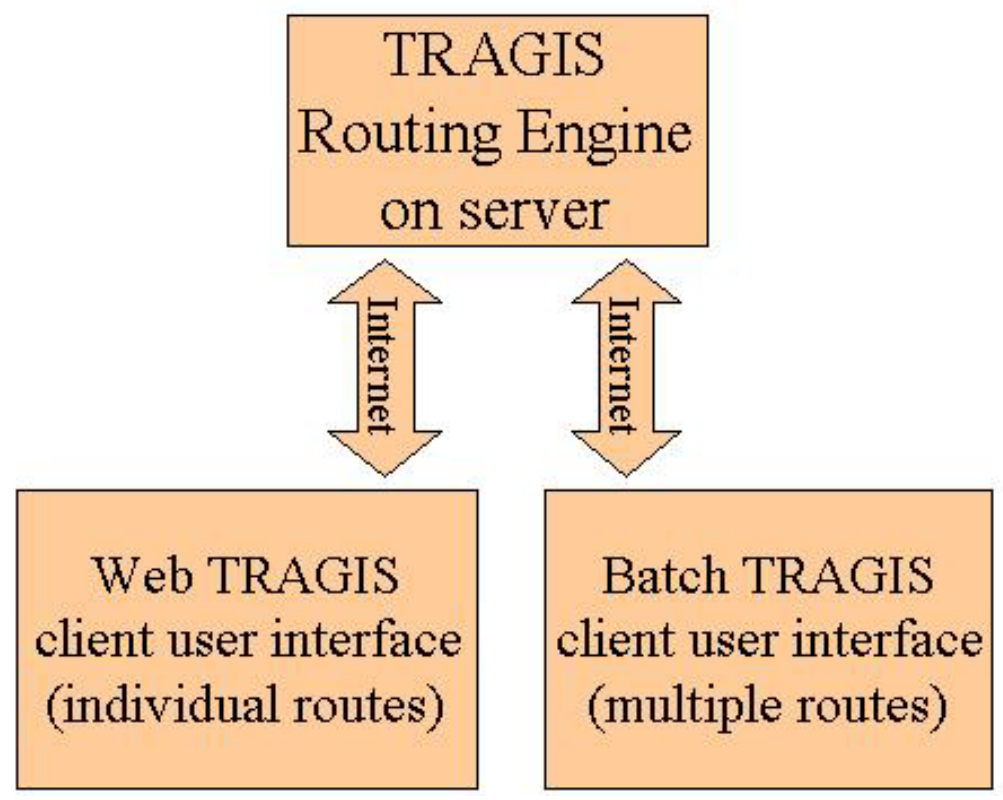

Fig. 1.1. Conceptual design of TRAGIS.

The lineage of TRAGIS can be traced back a quarter of a century to the development of two transportation routing models at Oak Ridge National Laboratory (ORNL) in 1979: HIGHWAY (Johnson 1993a), which predicts truck transportation routes, and INTERLINE (Johnson 1993b), which predicts rail and waterway transportation routes. Both of these models were used for many years by the DOE community for a variety of routing needs. The development of TRAGIS dates back to April 1994, when the DOE Office of Environmental Restoration and Waste Management's (EM) Transportation Management Division (EM-261) held a Baseline Requirements Assessment Session with transportation routing experts and users of the HIGHWAY and INTERLINE models. As a result of the session, the development of a new GIS routing model, TRAGIS, was initiated.

TRAGIS went through several steps in its early development. The initial version of the model operated on $\mathrm{SUN}^{\mathrm{TM}}$ UNIX $^{\circledR}$ workstations. At that time the Windows PC operating system could not easily handle the large geographic transportation networks in TRAGIS. The availability of TRAGIS only on UNIX 
workstations severely limited the availability of TRAGIS to its user community because very few users had such computers. The development of the current version of TRAGIS as a client-server web application was seen as the best option to vastly improve the accessibility of the model.

TRAGIS is developed to be accessible over the WWW with an easy-to-use Windows interface. The model is deployed as a client-server application, where the map data files and user interface software reside on the user's PC and the routing engine with its large data files reside on the server. TRAGIS deployment is handled through an access-controlled web page where the client software may be downloaded. The client software serves as the user interface to the routing engine. The routing engine, written in $\mathrm{C}++$, resides on the server and receives the route parameters across the Internet. On the server, the routing parameter inputs are passed to the routing engine, used to calculate a route, and then generates output listings as text files. The output files then are transmitted over the Internet to the user interface that displays the route. The user interface includes mapping features written with Environmental Systems Research Institute, Inc. (ESRI) MapObjects. The user interface operates quickly and efficiently over the WWW because the large map data files reside on the user's PC. The software is written to minimize the data transmission, so the speed of operation is virtually the same whether one is connected via a modem or a high-speed Internet connection.

WebTRAGIS works with highway, rail, and waterway routing networks. The geographic quality of the transportation networks is the aspect of TRAGIS that is vastly improved over the legacy HIGHWAY and INTERLINE models. With the legacy models, the transportation networks were essentially stick figure representations. This is sufficient for route calculations, but an accurate representation of transportation networks becomes important when GIS analysis is needed. An example of such a need is determining which Indian reservations a route passes through or overlaying urbanized areas on routes.

The goal for WebTRAGIS is to have national 1:100,000-scale routing networks. The highway network developed for TRAGIS is a 1:100,000-scale database. The legacy HIGHWAY model used a stick figure network with nodes digitized at 1:250,000-scale. The TRAGIS highway network was developed from the U.S. Geological Survey (USGS) Digital Line Graphs and the U.S. Bureau of Census Topologically Integrated Geographic Encoding and Referencing (TIGER) system. The rail network used in the initial version of TRAGIS was the same database as that used in the INTERLINE model. This network also was a stick figure network with nodes that were digitized from variable scaled maps. A 1:100,000-scale rail network is now incorporated into TRAGIS. The current inland waterway network is based on the USGS 1:2,000,000-U.S. Geodata. Deep-water routes are depicted in WebTRAGIS as straight-line segments. It is planned to incorporate a 1:100,000-scale waterway database into the model at a future time so that all modes will be at a consistent scale.

An important aspect of TRAGIS is the need to continually review and update the routing networks. Infrastructure changes occur with new road construction, highway renumbering, rail abandonment, and changes of rail ownership. The routing networks in the TRAGIS model undergo a scheduled maintenance to ensure that the quality of the databases is as accurate as possible.

Another feature of TRAGIS is a consistent user interface between the transportation modes. This was a problem with the legacy HIGHWAY and INTERLINE models. Each of the legacy models had variations within similar functions. With TRAGIS, functions are similar for running rail, highway, or waterway routes. Some variations occur, such as selecting from multiple railroad companies for rail routing. However, when a user learns one transportation mode of the TRAGIS system, it is not difficult to operate the other portions of the model. 
TRAGIS allows the selection of the origin and destination of a route from a list of node names. When selecting nodes, the program displays a list of state abbreviations from which the user selects a state. Next, a list of node names within that state is displayed. The user can scroll through this list and select a node. After a node is selected, TRAGIS displays the selected node's identification number. In addition to nodes at city locations and within the network, the TRAGIS databases contain hundreds of specific nodes for locations of all commercial nuclear reactors, DOE sites, military installations, and other important nuclear-related sites of interest. This user's manual includes appendixes of selected facility locations for both the highway and rail routing networks.

After an origin and a destination are selected, the model is ready to calculate a route based on criteria established by option selections. A default set of criteria is active for each transportation mode in the model. After completing the route calculation, TRAGIS displays the standard route listing. The user can also view a detailed listing of the route and population-density information, which can be used with the RADTRAN or RISKIND risk models, simply by selecting that button with a mouse click.

Option settings provide a mechanism to change various parameters used by the model for route calculations. Examples of some of the options include adjusting the penalty factors for the mainline classifications for rail routing, using preferred highway routes for radioactive materials, and running alternative routes for the different transportation modes in TRAGIS.

TRAGIS also provides functions to temporarily modify the routing networks. More experienced users may select individual nodes and links or an entire state in which all nodes and links are blocked from the network. In the rail portion, entire railroad companies can be blocked from consideration.

The primary capabilities available with TRAGIS are the GIS features and the ability to map calculated routes. The mapping capability was a feature that was unavailable with the legacy models and an enhancement that needed to be incorporated into the new model. WebTRAGIS provides the ability to generate, save, and print maps of routes.

One of the additional features available with TRAGIS is information on Native American Tribal Lands along routes. This data is only possible with such detailed shape files of the routing networks along with similar detailed tribal land boundary files. Tribal land boundaries are overlaid with the highway and rail networks allowing the model to report the names and the route mileage within each reservation. The tribal lands can also be viewed in relation to routes with the WebTRAGIS mapping features.

Population data for transportation routes has always been one of the main features of the ORNL routing models. Over the years, the techniques to process Census numbers have improved and with the 2000 Census, LandScan USA population analysis modeling is being used by the TRAGIS model. Also with the 1:100,000-scale highway and rail networks, the quality of the population density and counts reported by TRAGIS has improved dramatically with the ability to select from multiple buffer distances.

This user's manual has six sections following this introductory section. Section 2 provides installation instructions and a general overview of the TRAGIS model. This section is very useful for a user who has not worked with graphical user interface (GUI) programs. Highway, rail, and waterway features are discussed in Sects. 3-5, respectively. Section 6 describes the mapping capabilities and functions of the model. Finally, Sect. 7 briefly discusses future development plans of the model. 
Page intentionally left blank 


\section{GENERAL OVERVIEW OF TRAGIS}

TRAGIS is written to operate as a PC Windows application for Microsoft Windows 98 or later operating systems. The software is distributed as a downloadable client-server application from the TRAGIS home page at http://apps.ntp.doe.gov/tragis/tragis.htm. Figure 2.1 shows the TRAGIS home page. Several links are provided on the lower portion of the TRAGIS home page.

- Installation Instructions provides a short description of the set to install the model on your PC.

- User Manual allows access to an Adobe Acrobat version of this document.

- User Registration provides instructions and the form for registration to become a TRAGIS user.

- Login allows access to download the TRAGIS software after your user registration is approved.

- Disclaimer provides information that all users should read regarding use of Federal computer systems.

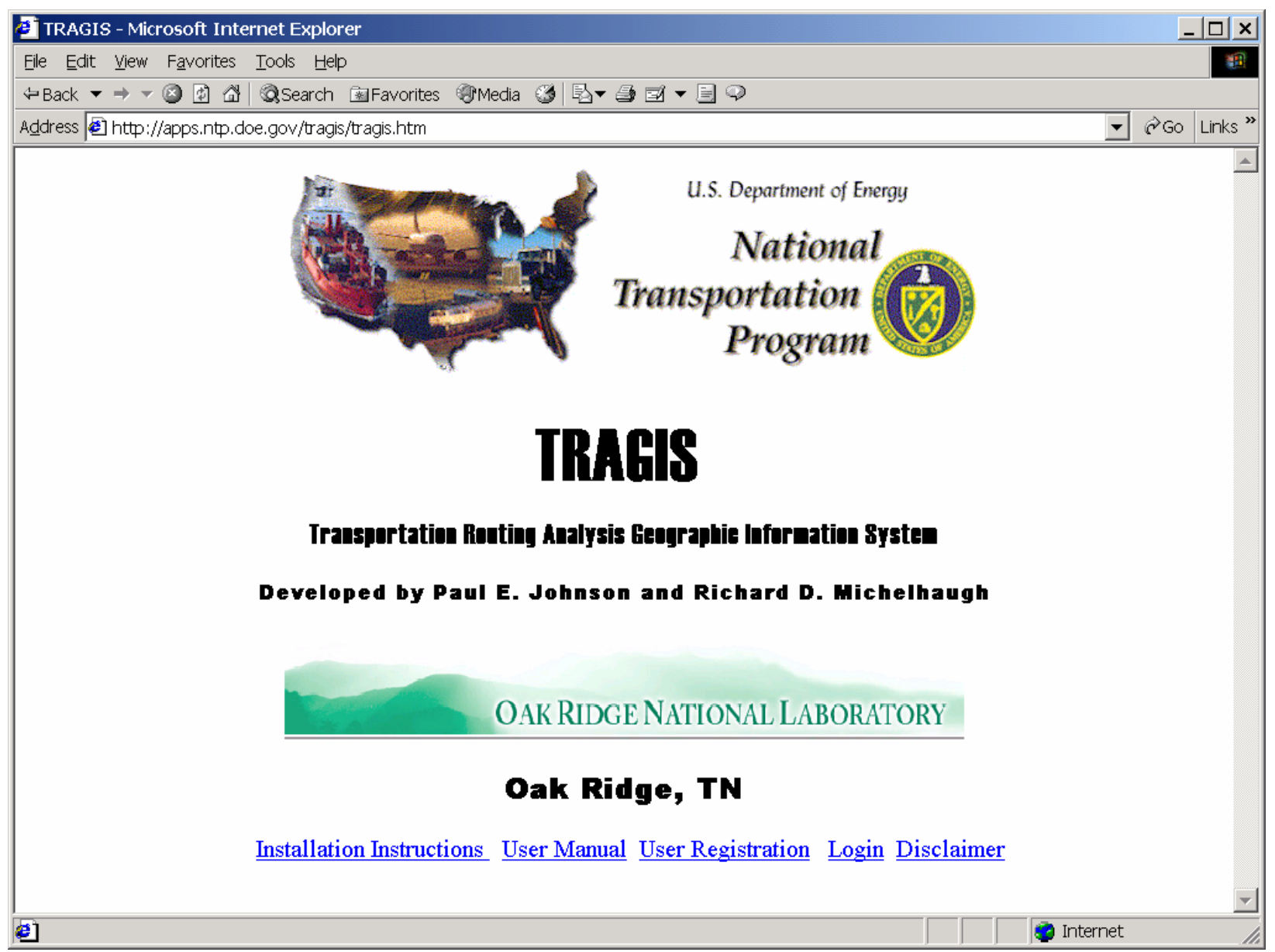

Fig. 2.1. TRAGIS web site home page.

\subsection{REGISTERING FOR TRAGIS}

The first step for a new user is to register for TRAGIS by clicking on the User Registration link at the bottom of the TRAGIS home page, as shown in Fig. 2.1. The registration page contains the instructions 
and a set of records that need to be completed by the prospective user. Figure 2.2 shows the fields that the new user needs to complete. Data should be entered in all fields, especially the reason for your need to access the TRAGIS model. Currently, the user is allowed to select their user name and password. The system will not allow a new user to select a three-letter username that is already in use. So if problems are experienced, a new username should be tried. The password should be at least eight characters long and include a mixture of letters and numbers. As with any computer password, use a common phrase or fixed set of numbers is not advisable. Potential users need to be aware that this model can only be used for government projects and commercial users will not have their registrations approved by the TRAGIS project manager. Click on the Disclaimer link on the TRAGIS home page to read the entire disclaimer notice.

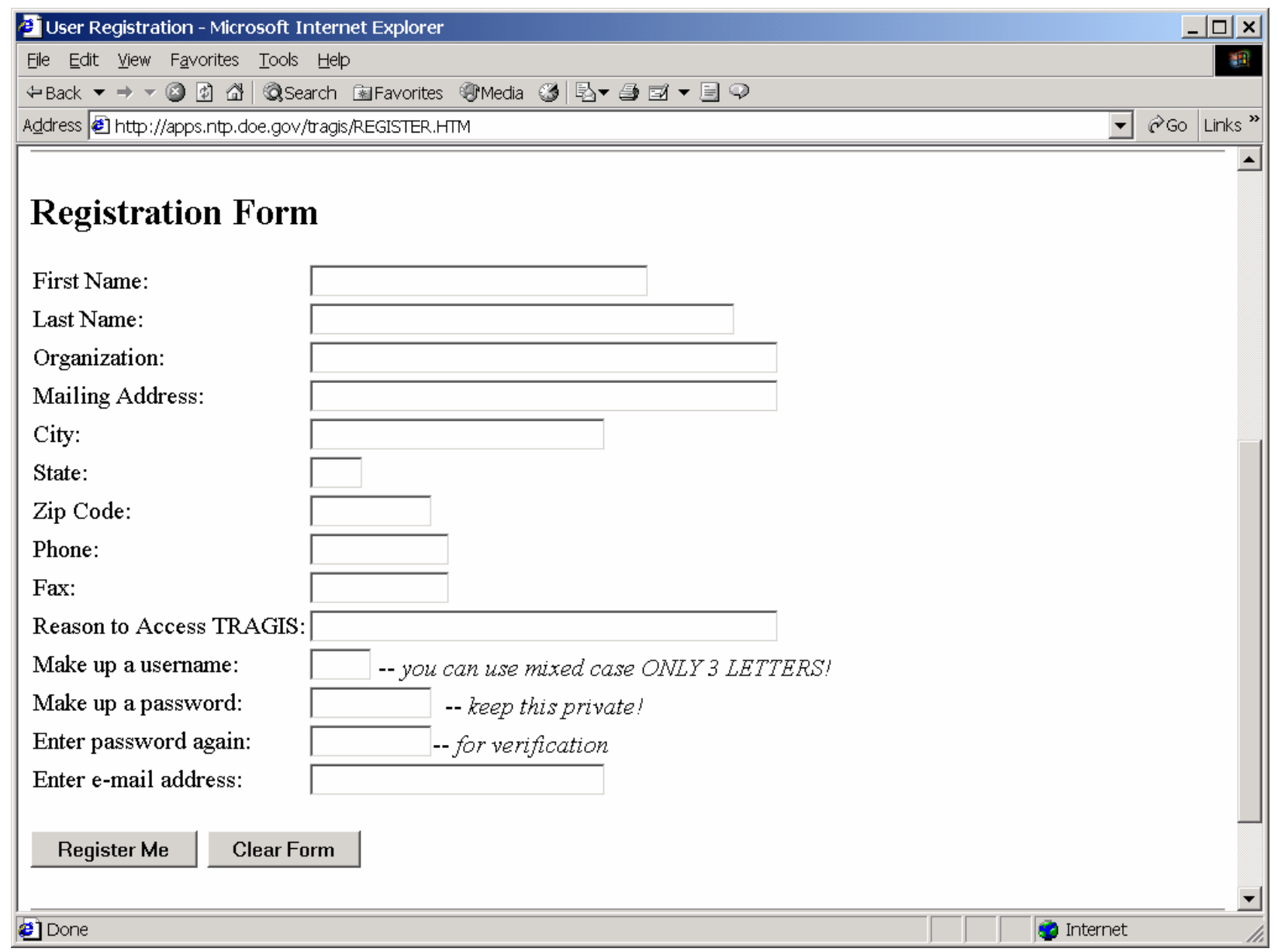

Fig. 2.2. TRAGIS user registration form.

Upon completing the user registration, the system generates an e-mail message to notify the TRAGIS project manager and developer that a new account is pending approval. After TRAGIS management reviews the registration information, an e-mail message is sent to the user notifying them whether their account is approved or rejected.

\subsection{INSTALLING WEBTRAGIS}

After being notified of registration approval, the new TRAGIS user can return to the TRAGIS home page and click the Login link to download either the WebTRAGIS or BatchTRAGIS software. (At the writing 
of this manual, BatchTRAGIS is still in development and is not yet available to the TRAGIS user community.) The next screen requests the user's three-character username and password. Enter the proper information and press the OK button. Following this selection, the WebTRAGIS Client Setup appears in the web browser, as shown in Fig. 2.3. As shown in this figure, the size of the downloadable file is about 51 megabytes in size, which may be impractical to download with a slow modem connection. (If your Internet connection speed makes it impractical to download a file of this size, please contact TRAGIS management staff to request a compact disk copy of the client software and installation instructions.) Click on the Install button on the screen, as shown in Fig. 2.3.

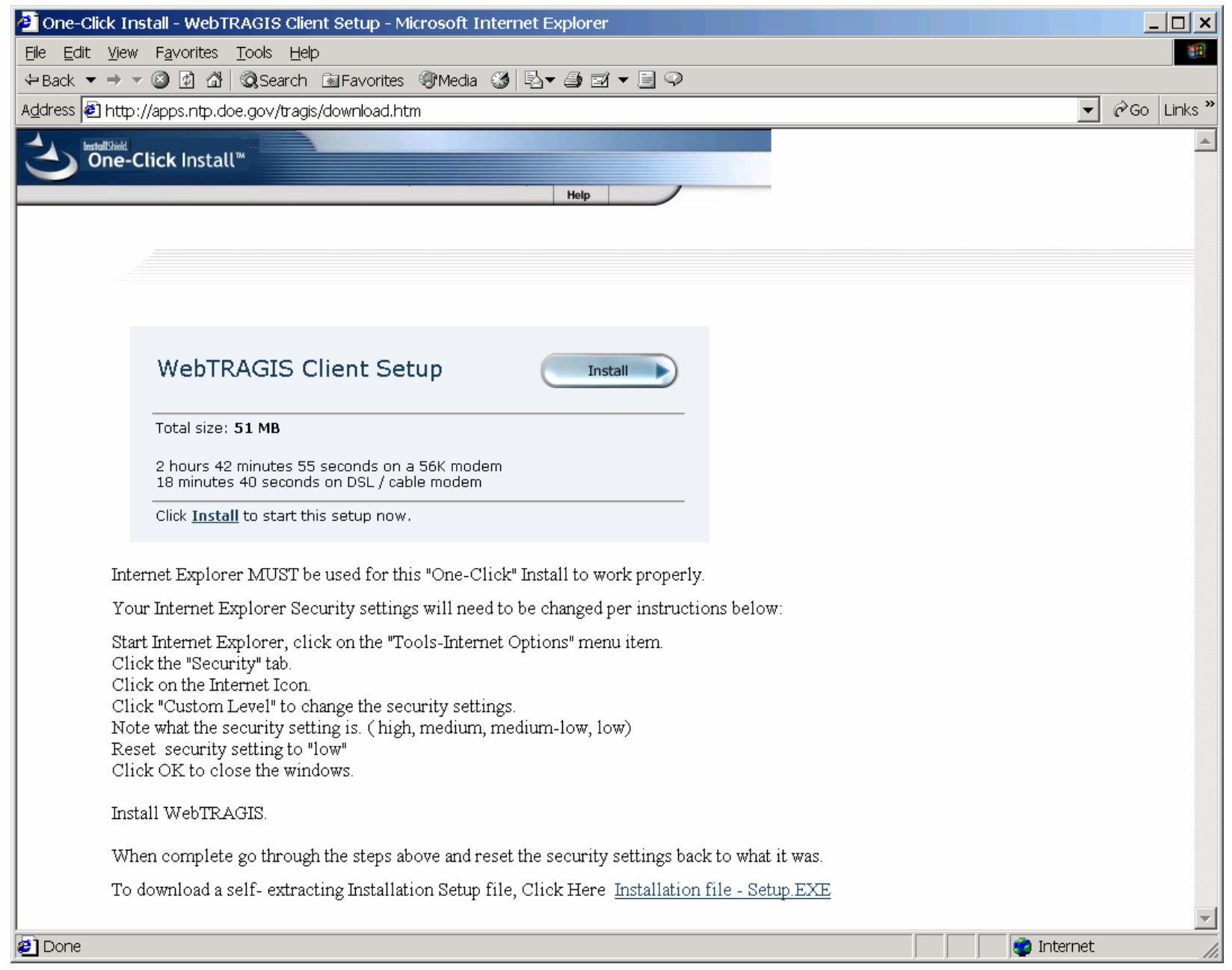

Fig. 2.3. WebTRAGIS client setup screen.

During the installation process, a security warning message, such as shown in Fig. 2.4 may appear. If this warning message is displayed, press the Yes button to continue the installation. A status window will be displayed showing the progress of downloading the installation wizard file. The amount of time required to download the file should not take too long, but this depends upon the user's Internet connection and the volume of traffic on the network. 


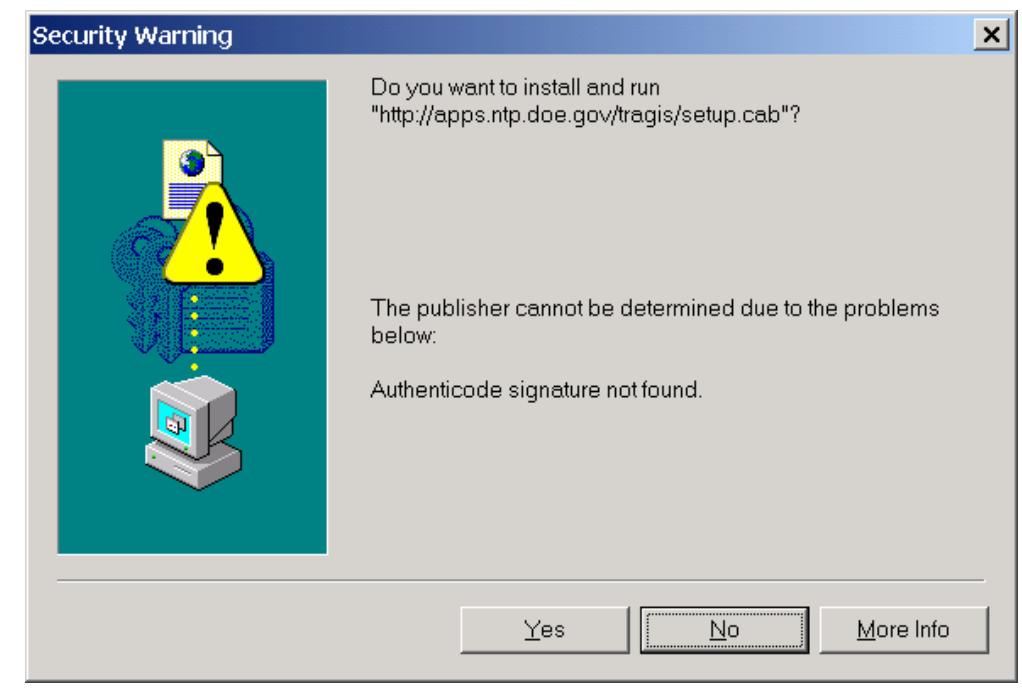

Fig. 2.4. Security warning screen during WebTRAGIS installation.

After the download process is completed the InstallShield Wizard for the WebTRAGIS Client starts and displays a window as shown in Fig. 2.5. To proceed with the installation of the WebTRAGIS client on your PC, press the Next button at the lower portion of the installshield wizard screen.

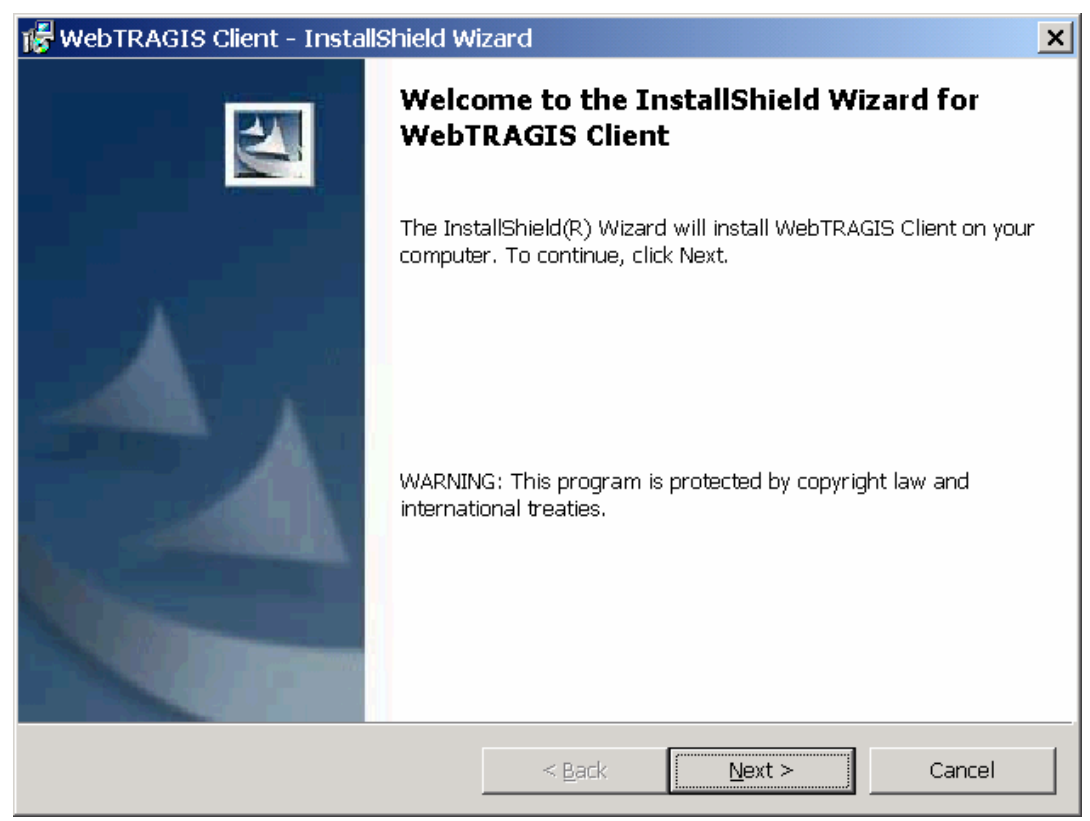

Fig. 2.5. The InstallShield Wizard for WebTRAGIS Client window.

Next, a verification screen shows the location of the destination folder and user information for the installation. Figure 2.6 provides an example of this screen. The InstallShield Wizard is set to install WebTRAGIS in the C:|Program Files $\backslash$ WebTRAGIS $\backslash$ folder, but allows for the selection of another drive. To proceed with the installation, click on the Install button. A status window will then appear and show the progress of the installation. 


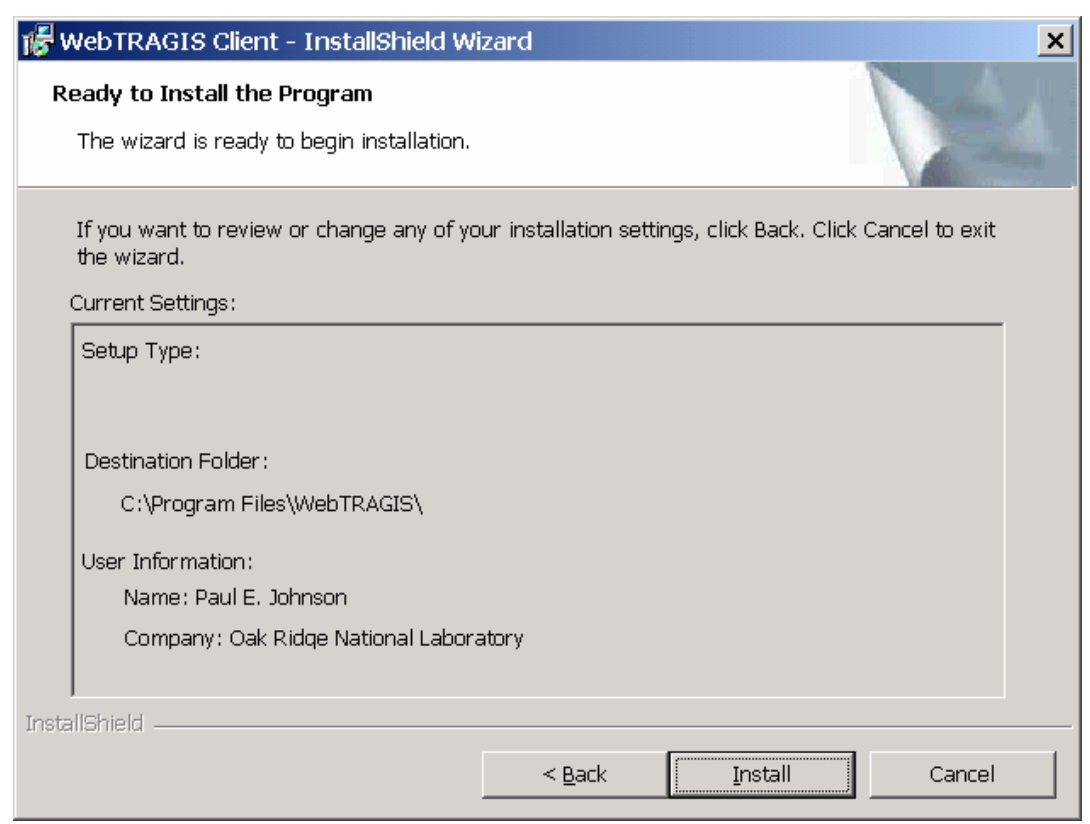

Fig. 2.6. Verification of the installation settings for WebTRAGIS client.

When the InstallShield Wizard program completes the installation of the WebTRAGIS client, a window, as shown in Fig. 2.7, is displayed. To complete the installation process, click on the Finish button. The installation program removes all temporary files on your PC.

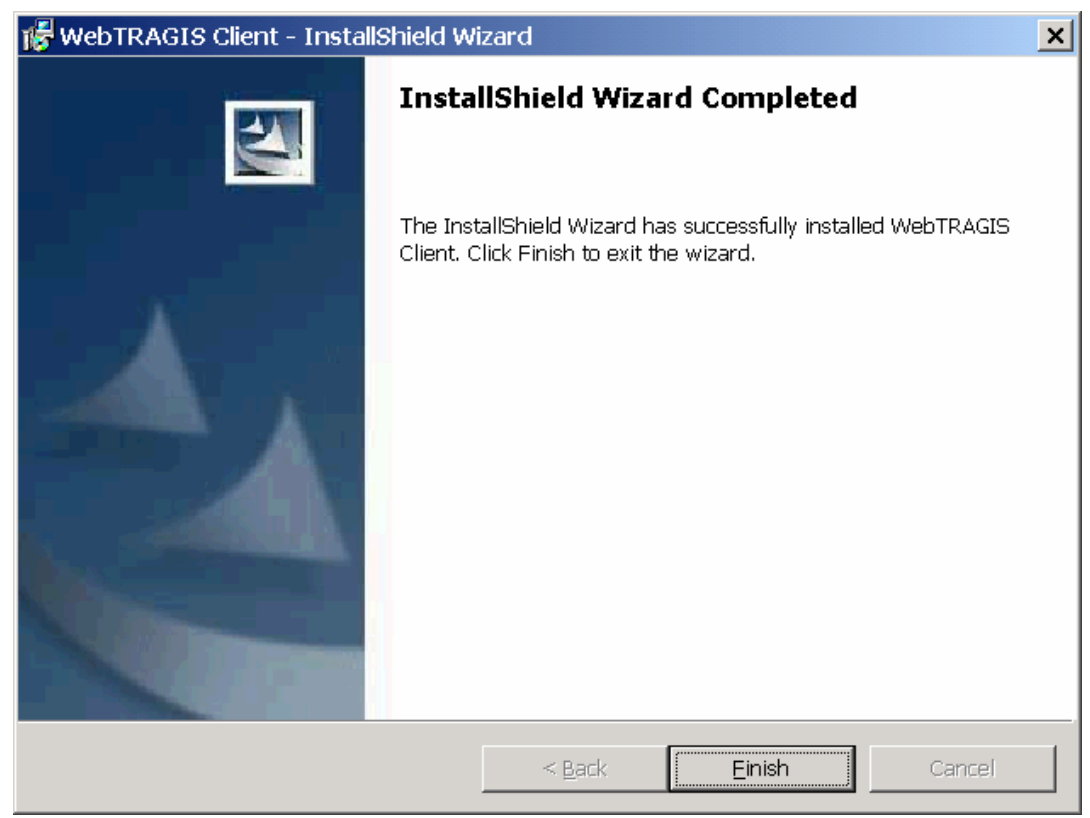

Fig. 2.7. Verification window indicating successful installation of the WebTRAGIS client.

\subsection{STARTING WEBTRAGIS}

To start WebTRAGIS, click on START $\rightarrow$ Programs $->$ WebTRAGIS $->$ WebTRAGIS Client. After loading, the model will display a username window, shown in Fig. 2.8, which is used to control user 
access to the WebTRAGIS user interface and routing engine. Enter your three-character username and password in this window and press the start button. It will take a few seconds for WebTRAGIS to connect across the Internet to the databases needed for the model.

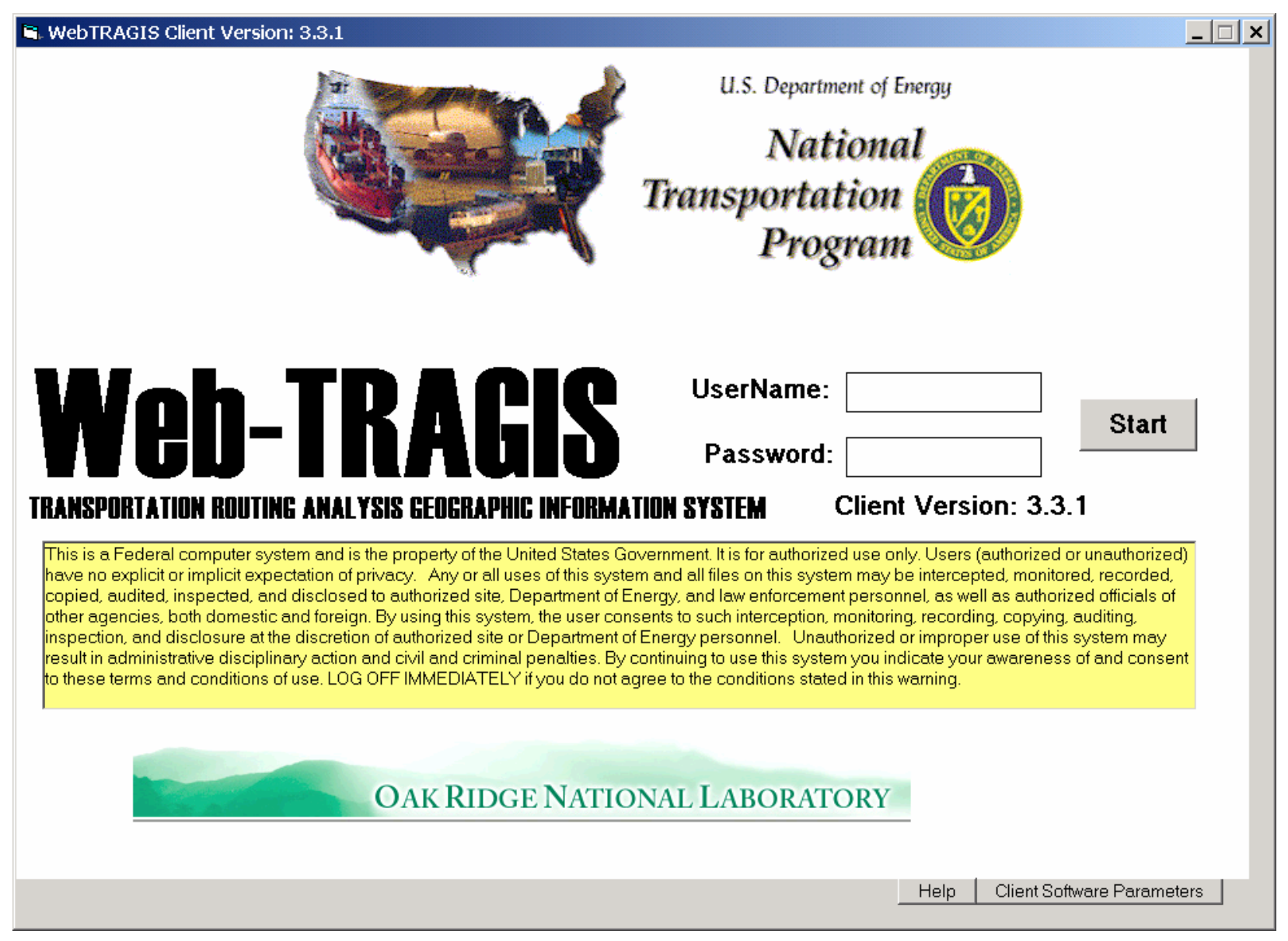

Fig. 2.8. WebTRAGIS login screen.

\subsection{WEBTRAGIS LAYOUT}

Figure 2.9 shows this initial appearance of WebTRAGIS after the user has entered their username and password. WebTRAGIS is designed with a number of different functional panels accessed by clicking on the screen tabs at the top of the window. The program initially opens to the Select Origin and Destination screen with the Highway mode selected.

There are six screen tabs on the WebTRAGIS program screen-4 screen tabs for selecting routing parameters, a screen tab for viewing output files, and a screen tab for generating and viewing maps. Each of these screen tabs is discussed in general terms below. A detailed description of each of these screen tabs appears later in this document in the respective chapters for each transportation mode.

Two additional buttons are located at the lower right corner of all the tab screens. The Help button provides telephone numbers of TRAGIS team members. The Client Software Parameters button is primarily a debugging tool that provides details about the connections to the TRAGIS web server. 


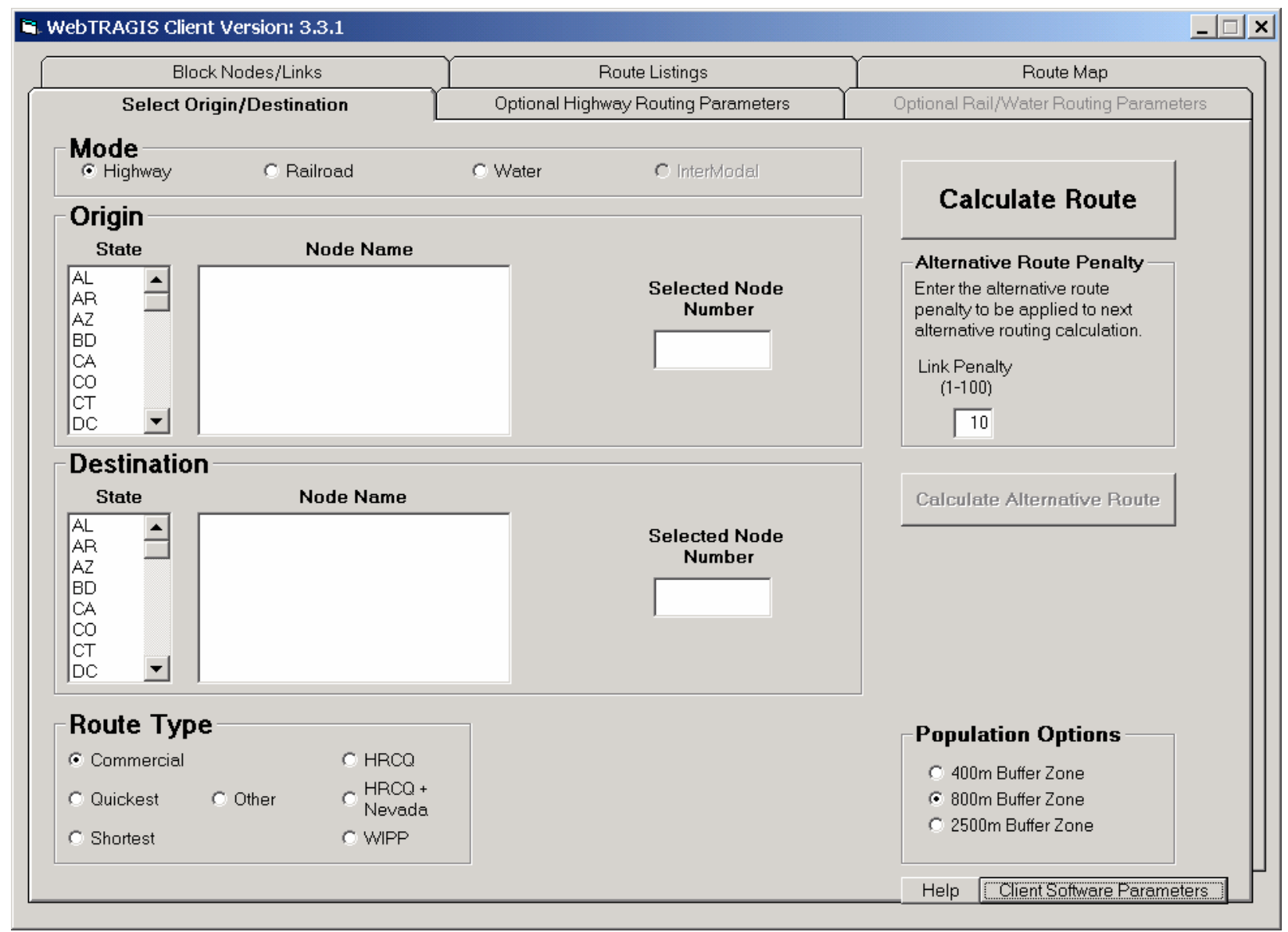

Fig. 2.9. WebTRAGIS initial screen after login.

\subsubsection{Select Origin/Destination Screen Tab}

The Select Origin/Destination screen tab is the initial screen displayed following successful entry into WebTRAGIS. This screen has six different boxes for a number of selections related to route selection along with the calculate route and calculate alternative route buttons.

Mode selection is the first box along the upper edge of this screen. By default, WebTRAGIS initially selects the highway mode. Other modes can be selected by clicking on either the respective radio button or mode name. After a different mode is selected, the system may take several seconds to reset the screen format to that mode. The appearance of this screen varies slightly depending upon the selected transportation mode. At this time, the intermodal feature of WebTRAGIS is not active.

The remainder of this screen consists of boxes for the selection of the origin, destination, route type, population options, and alternative route penalty. Layouts of these boxes are similar, but may vary with the particular mode that is selected. The boxes have a default value selected, so only an origin and destination need to be selected before the calculate route button can be pressed. A more detailed description of each of these items is provided in the respective transportation mode chapters. 


\subsubsection{Optional Highway Routing Parameters Screen Tab}

The Optional Highway Routing Parameters tab is active when WebTRAGIS is set to the highway routing mode. This screen tab contains seven optional routing parameters. They include driver options, highway inspection, date and time options, toll-bias factor, Nevada county population details, road-lanetype penalty, and other constraints. A full explanation of these options is provided in Sect. 3.5.

\subsubsection{Optional Rail/Water Routing Parameters Screen Tab}

The Optional Rail/Water Routing Parameters tab is active when WebTRAGIS is set to either rail or waterway modes. This screen tab contains four optional routing parameters for railroads and three for water routing. The four railroad option boxes control the originating railroad factor, rail line type weighting, rail transfer penalty, and Nevada county population details. The three water option boxes are select water route type, water weighting factors, and modify mode transfer penalty. A full explanation of these options is provided in Sects. 4.6 (for rail options) and 5.5 (for water options).

\subsubsection{Block Nodes/Links Screen Tab}

The Block Nodes/Links tab contains a number of different blocking options for the transportation networks. Blocking action results in a temporary modification of network nodes and links to allow the user to avoid passing through a particular part of the transportation network. All transportation modes in WebTRAGIS have the block nodes and block links options. With the highway and rail networks, individual states can be selectively blocked. The rail network includes a feature to block individual railroad companies.

\subsubsection{Route Listings Screen Tab}

The Route Listings tab screen allows the user to view and print the various files created and used by WebTRAGIS. After completing a route calculation, this screen is displayed showing the standard route listing. The information shown on this screen is controlled by a series of buttons found along the upper portion of the display. The buttons on this screen are:

- Standard Listing button displays the normal route description of the most recent route calculated. (WebTRAGIS displays this listing following the calculation of a route.)

- Standard and Pop Listing button displays the combination of what is shown with both the Standard Listing and Population Data Listing buttons.

- Detailed Listing button displays a listing that includes all named nodes along a route. For long routes, this listing can be rather lengthy.

- Population Data Listing button displays the population density table, RADTRAN input data for the route, and the population counts within the specified route buffer.

- Print button sends the information listed in the screen to a printer.

- Save As button saves the information listed in the screen to a file.

- Route Info button displays the most recent set of parameters used to create a route (such as the origin, destination, option settings, blocked nodes and links, etc.).

- ReCalc Route button will recalculate a previously saved route info file. This feature is primarily used to replicate user problems by TRAGIS development staff.

- Map Info button displays the beginning and ending coordinates of each individual link for the most recently calculated route. This screen only provides node coordinates and does not provide the shape coordinates for the links. Information on this screen is used to draw the straight-line segments in the route map screen discussed in the next section. 
- Error Log button displays any error messages that the model generates. The model will automatically display an error message if TRAGIS encounters a problem.

- Clear Output button will remove the contents of all output files (the standard listing, detailed listing, population data listing, and map info files).

\subsubsection{Route Map Screen Tab}

The Route Map tab provides the mapping functionality for WebTRAGIS. This portion of WebTRAGIS uses ESRI MapObject software. In the upper right portion of this screen are four buttons used to display and clear routes. WebTRAGIS provides two types of mapping; straight line segments and shape files.

The first is a fast and simple route display, which involves representing routes as straight-line segments between nodes. This method is fine when generating a national view of the route, but is not very good when creating a large-scale map for a small geographic area. The three upper buttons at the upper right portion of this screen deal with the straight-line routes. By pressing the Show Route button, the most recently calculated route is displayed. To remove the displayed route, press the Clear Routes button. The Save Route button will save all the routes currently displayed on the screen in an ESRI shape file.

The Generate Shape File button generates an ESRI shape file with the same curvature as shown in the full network shape file that is shown as a background on the maps. When the Generate Shape File button is pressed, a Save As File window is displayed and the user can select the file name and location where the shape file is saved on their computer.

To save a view of the map shown in the Route Map screen, press the second button from the left that has an image of a diskette. This Save Displayed Map to Graphics File button will save the display as either an enhanced metafile (.emp) or bitmap (.bmp) format file.

Section 6 of this user's manual fully describes the button bar and other mapping features of WebTRAGIS.

\subsection{TRAGIS POPULATION DATA}

An important feature of the TRAGIS model is the ability to obtain detailed route-specific population density and count data for calculated routes. The population data in TRAGIS is derived from the ORNL developed LandScan USA 15 -arc second (approximately 360 by 460 meter) grid cell population database (Bhaduri 2002). This national database represents nighttime population distribution and is developed from a combination of data sources including 2000 U.S. Census Bureau block group population, roads from the Census TIGER data, slope from the National Imagery and Mapping Agency's (NIMA) Digital Terrain Elevation Data (DTED), and land cover from the USGS National Land Cover Database (NLCD). The data are modeled to best approximate the actual location of the resident population.

Each grid cell represents a specific number of people. The modeled data is normalized to the block group. This means that for a specific Census block group, the grid cells that comprise or cover the area represented by the block group have the total population of that block group. The geographic area of each grid cell is also known and the population density of each cell is computed by dividing the population of a cell by the area of the cell. To calculate the population density, buffers are built around the transportation links and the geographic area for each of the population density categories is summed. These data are then converted to represent the mileage of the route in each of the population density categories shown in the population density table available in TRAGIS. Population counts are determined by summing the population of the cells with the buffer around the transportation links. 
Figure 2.10 provides an example of the LandScan USA 15-arc second data in the Oak Ridge and west Knoxville area. Various highways and railroads are labeled on this map along with several communities. It is easy to see the individual grid cells, especially in areas where there is variation in population. The figure provides a legend indicating the TRAGIS highway and rail networks in the area, along with the range of values within each of the population categories. Each cell actually represents a finite number of people. It is easy to identify the DOE Oak Ridge Reservation, the large area with no population where the ORNL and Y-12 sites are located. Also, the commercial developed areas of Oak Ridge along State Route 95, northeast of State Route 62 are visible. The Clinch River can be easily seen along the CSXT rail line east and north of the Solway community.

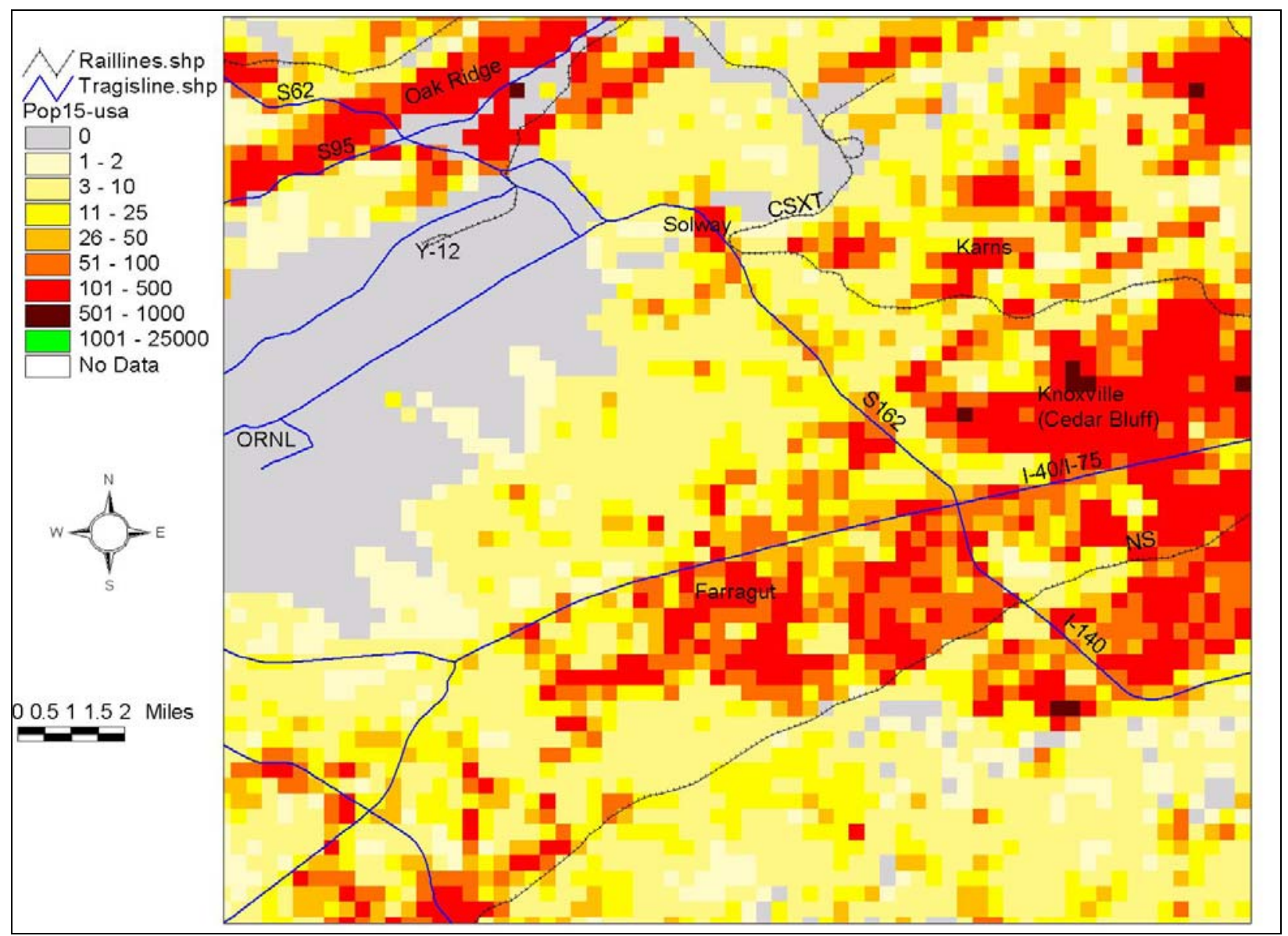

Fig. 2.10. Grid cell population data and transportation networks.

Population density and count information is based on geographic buffers constructed around the transportation route. This information is not calculated by the TRAGIS model, but has been precalculated for all links in each transportation network. Data on three different buffer distances are available in TRAGIS - 400, 800 and 2500 meters either side of each link. After a route is calculated, TRAGIS sums the population density and count data for the links on that route. The population data for each route is accessed with the Population Data Listing button on the Route Listings screen tab.

Figure 2.11 shows the buffers for the highway and rail links for the same east Tennessee area. By comparing Figs. 2.10 and 2.11, the reader can visually see the grid cells that comprise a particular link buffer. GIS techniques are used to calculate the population density and count for each link. It needs to be noted that a special technique is used to avoid double counting of population where links overlap at node 
locations. This situation is best viewed along I-40/I-75 at the junction with the Pellissippi Parkway (I-140 and State Route 162) in the right center of Fig. 2.11. The four links all meet at this node location. A circle with the radius of the buffer distance represents the overlap of the four links. The population within this radius for each node is only counted once during the summation of the population along each route to avoid the double counting the people in the vicinity of the node.

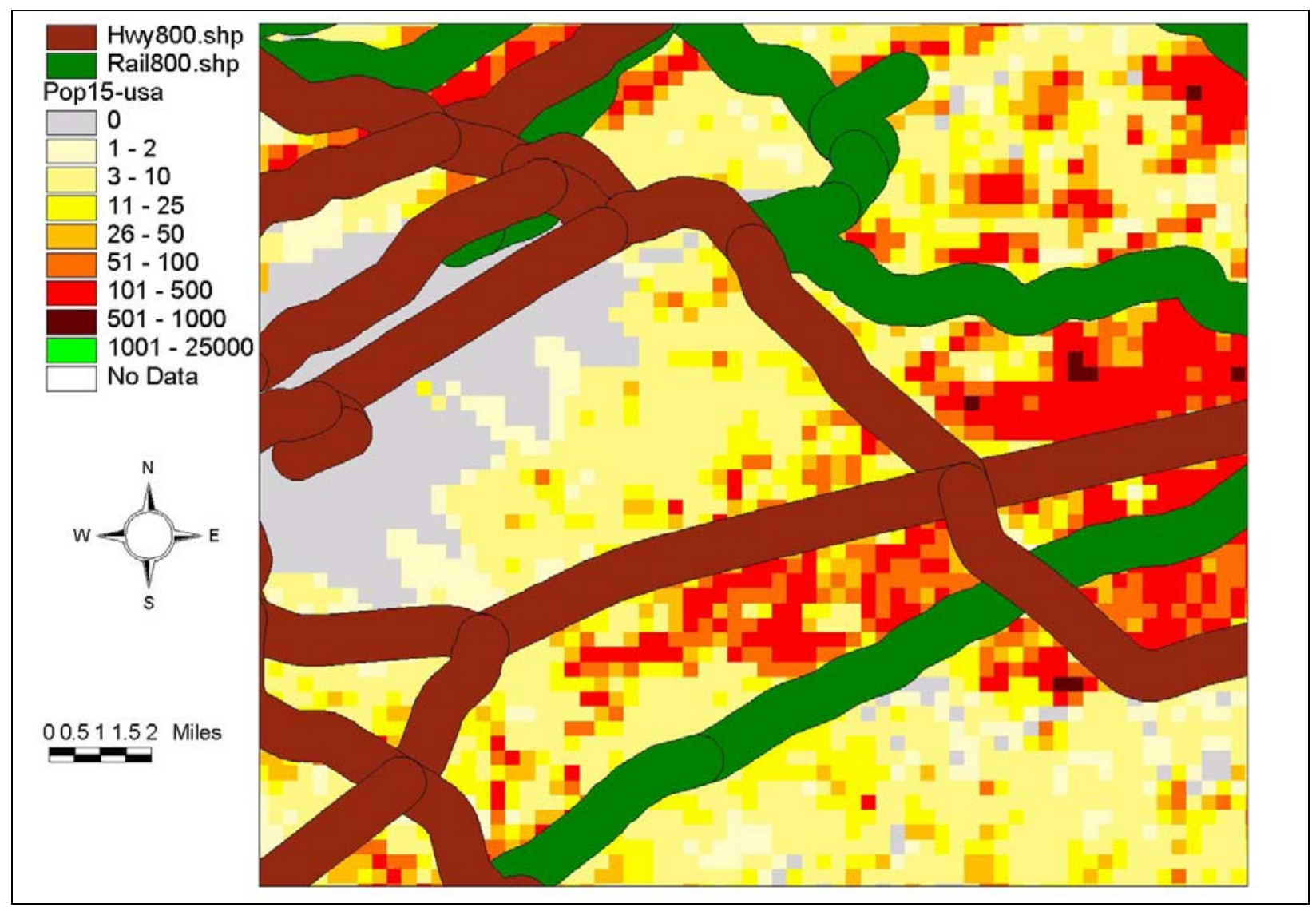

Fig. 2.11. Buffered (800 meters either side) highway and rail lines.

ORNL is currently developing higher resolution population density information. As this document is written, the counties containing the largest cities in the country are being processed using Census block data, the smallest geographic level of detail available. These data are being processed at a 3 arc-second (approximately 90 meter) grid cell level, or 25 times the resolution of the current population data in TRAGIS. Data are also being developed for daytime population. It is unknown when this data would be developed for the entire continental U.S. so that it could be incorporated into the TRAGIS model. 
Page intentionally left blank 


\section{HIGHWAY ROUTING}

The TRAGIS model can calculate a number of different types of highway routes. By default, the model calculates commercial highway routes; but with the change of the route type, the model can determine routes that meet the U.S. Department of Transportation (DOT) regulations for shipments of highway route-controlled quantities (HRCQ) of radioactive material, routes for shipments to the Waste Isolation Pilot Plant (WIPP), the shortest, or the quickest route.

\subsection{HIGHWAY NETWORK}

The TRAGIS highway network is developed from a 1:100,000-scale road network derived from USGS digital line graphs and Census TIGER data. The network represents slightly over 235,000 miles and includes all Interstate highways, most U.S. highway except those that closely parallel Interstate highway, major state highway, and other local roads connecting to various specific sites of interest. Currently there are over 22,000 highway segments (links) and over 16,000 intersections (nodes). Included in the network are all commercial nuclear power plants, DOE sites, commercial airports (designated by their location identification code), and military airports (also designated by their location identification code).

Appendix A provides a list of node names for selected facilities in the TRAGIS highway network. A GIS distance measuring technique using an equidistant projection calculates the mileage of each link in the highway network. This is an improvement over the legacy HIGHWAY model where links were represented as straight-line segments and distances were manually determined from various map sources. Other attributes in the highway network include the speed limit, toll designation, commercial truck restrictions, roads within urbanized areas over 100,000 people, preferred route designation for HRCQ shipments, and non-radioactive hazardous material route restrictions.

\subsection{HIGHWAY NAMING CONVENTIONS}

The naming conventions used in the TRAGIS highway network have given some users difficulty. In the following sections, a description is provided for link and node names.

\subsubsection{Highway Link Naming Conventions}

The naming convention for road links in the TRAGIS highway network is comprised of up to five characters. The first character is a letter designating whether the highway is an Interstate, U.S., state, turnpike, county, or local road. The second through fifth characters represents the route number of the highway. In cases where roads do not have numbers, a short abbreviation of the road name is used. Names of toll roads and toll bridges include the $\$$ and \# signs, respectively, as the fifth character in the highway name. Examples of this convention are as follows:

- Interstate highways (e.g., I40, I35W, I470\$ \{toll road\}, I278\# \{toll bridge\});

- U.S. highways (e.g., U1, U31, U412);

- State highways (e.g., S9, S62, S162, S3132);

- Turnpikes that are not part of the Interstate System (e.g., TBGP \{Blue Grass Parkway in Kentucky\}, TFLT\$ \{Florida Turnpike toll road\});

- County roads (e.g., C2, C10, C300E);

- Local roads (e.g., LOCAL). 
Each link can have up to three different highway numbers assigned in cases where multiple highways traverse the same segment of a road. Examples include I90/I94 in Wisconsin, U14/U16/U20 in Wyoming, and S22/S33 in Idaho. Normally, mixtures of different sign typed roads are not included together, such as a U.S. highway running concurrent with an Interstate highway. The reason for not mixing the different sign types is to reduce the length of the standard route listing. In the route listing, only the first two road names are provided.

\subsubsection{Highway Node Naming Conventions}

Highway node names follow a standard naming convention consisting of three parts - a city name, a directional modifier, and a pair of intersection descriptors. The city name of the node is generally the city or town nearest the intersection. The directional modifier describes the direction that the node is in relation to the center or downtown portion of the city. Intersection descriptors normally identify the two primary highways that meet or cross at the node location. On most Interstate highway nodes, the Interstate number along with the exit number comprises the intersection descriptors. A node where two Interstate highways meet will have both Interstate highway numbers as the intersection descriptors.

Some nodes do not follow the standard naming convention. One situation is a node named for a facility, such as an airport, nuclear plant (NP), or DOE site rather than a specific city. Usually such facilities will not include a directional modifier or intersection description in the full name. The other situation is state border nodes. State border nodes are placed in a unique state designation in the TRAGIS highway network - a special state designation with the abbreviation of "BD," for border. The names of the state border nodes are constructed from the names adjacent nodes on either side of state line. The first four characters are the two state abbreviations of the bordering states, with the lower alphabetic state appearing first. The first four characters of the highway designation of the road crossing the state border follow the state abbreviations. The final eight characters of a state border node name consist of the first four characters of the city name of the adjacent nodes in either state, appearing in the same order as the state abbreviation order.

Figure 3.1 shows a portion of the highway network in the vicinity of Memphis, Tennessee. This figure assists in demonstrating the link and node naming conventions. First, some background information needs to be provided. The roads in this example are color-coded by the type of highway: Interstate highways are blue, U.S. highways are red, state highways are green, and local roads are orange. Black circles indicate nodes. The nodes in Tennessee and at the state border are all identified by their name.

It should be noted that all but three nodes in Fig. 3.1 are named Memphis. All of the Memphis nodes have directional modifiers, with the sole exception being the node located nearest to downtown Memphis. The directional modifier indicates the direction the node is from the downtown area of the city. Two nodes are located to the north, three nodes to the south, two to the southeast, and one to the east.

The intersection descriptors for the nodes in Fig. 3.1 show the two variations of the descriptors for Interstate highways. Three of nodes along I 240 are junctions between two Interstate highways. The intersection descriptors for these three nodes (Memphis I240, I40; Memphis S I240, I55; and Memphis E I240, I40) include I240 and the other Interstate highway number as the second intersection name. The other Interstate highway intersection descriptors have the Interstate highway number as the first intersection name and the exit number as second part of the intersection name. The letter " $\mathrm{X}$ " precedes exit numbers. Examples include Memphis N I40, X2A and Memphis S I55, X7. The Memphis N U51, S300 node represents a non-Interstate highway node intersection descriptor. As shown by this node, the two primary highways meeting at this node location are represented in the intersection descriptor. The 
hierarchy order for which highway is listed first is based on the order of highway types given in Section 3.2.1.

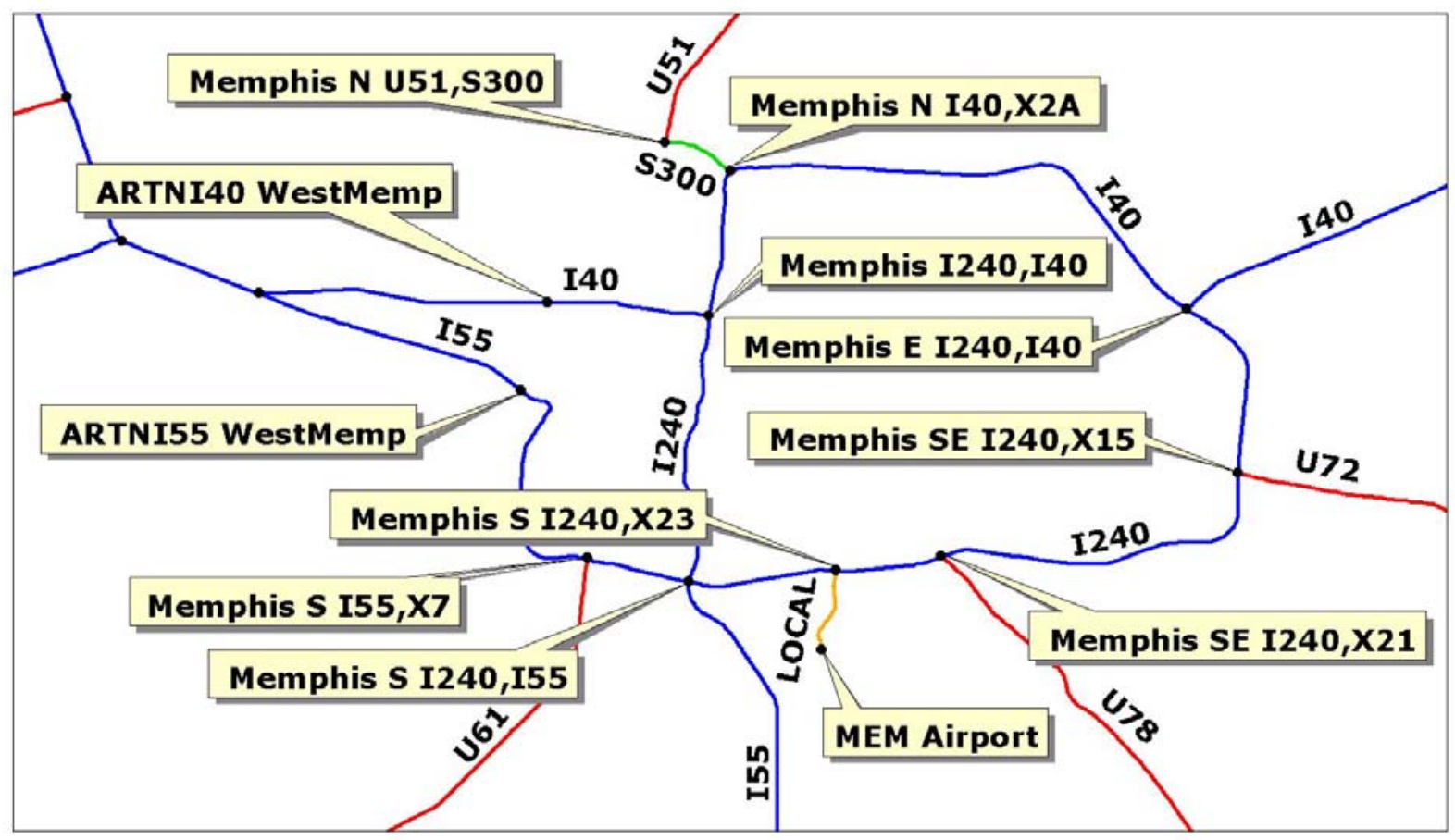

Fig. 3.1. Highway link and node naming example.

Some nodes do not have any directional modifier or intersection descriptors. One such node is "MEM Airport" on the south side of Memphis. This node represents the Memphis International Airport. The TRAGIS highway network includes locations for over 900 airports that have scheduled passenger service, military airports, or airports that have had DOE air freight shipments. Airports are identified by their three- or four-character standardized location identifier code. Other locations such as DOE facilities and commercial nuclear power plants are other sites that may not have a directional modifier or intersection descriptors as part of the node name.

Two border nodes are shown in Fig. 3.1 (named ARTNI40 WestMemp and ARTNI55 WestMemp). As described above, the names are a combination of the two border states (AR and TN), the highway name (either I40 or I55), and the first four characters of the adjacent nodes. The first node into Arkansas at the junction of I40 and I55 is not labeled in Fig. 3.1, but its name is West Memphis E I40, I55. Both node names to the east of the border nodes in Tennessee start with Memphis. Thus each of the two border nodes shown in Fig. 3.1 end with the characters "WestMemp." Knowledge of the border node nomenclature is useful for specialized techniques within TRAGIS, such as blocking all but one node along a state line to force the model to generate a specific route pattern.

\subsection{SELECT ORIGIN/DESTINATION SCREEN}

The TRAGIS model starts with the Highway mode selected and the highway network loaded. Figure 3.2 shows the display of WebTRAGIS in highway mode. The Select Origin/Destination screen allows the selection of the origin and destination, route type, population buffer zones, and the calculation of routes and alternative routes. Each of these features are discussed in the following sections. 


\subsubsection{Selecting an Origin and Destination}

Two methods are available for selection of an origin and destination of a route. The primary method is the selection from scrollable lists on the Select Origin/Destination screen. The alternative method, the manual entry of a node number, is available but is only used by power users.

\subsubsection{Primary method-selection from scroll box}

In TRAGIS, the primary method of selecting an origin and destination for a highway route is to select the node name within the respective scroll boxes. First scroll through the State list and highlight the desired state by clicking on its abbreviation. Upon selection of a state, node names within that state appear in the Node Name list. Scroll through the list and select the desired node name. After choosing a node name, the highway node number is displayed in the respective Selected Node Number box. The order for selection of the origin and destination does not matter. Once both are selected, the user may either press the Calculate Route button or select other user parameters to modify the route.

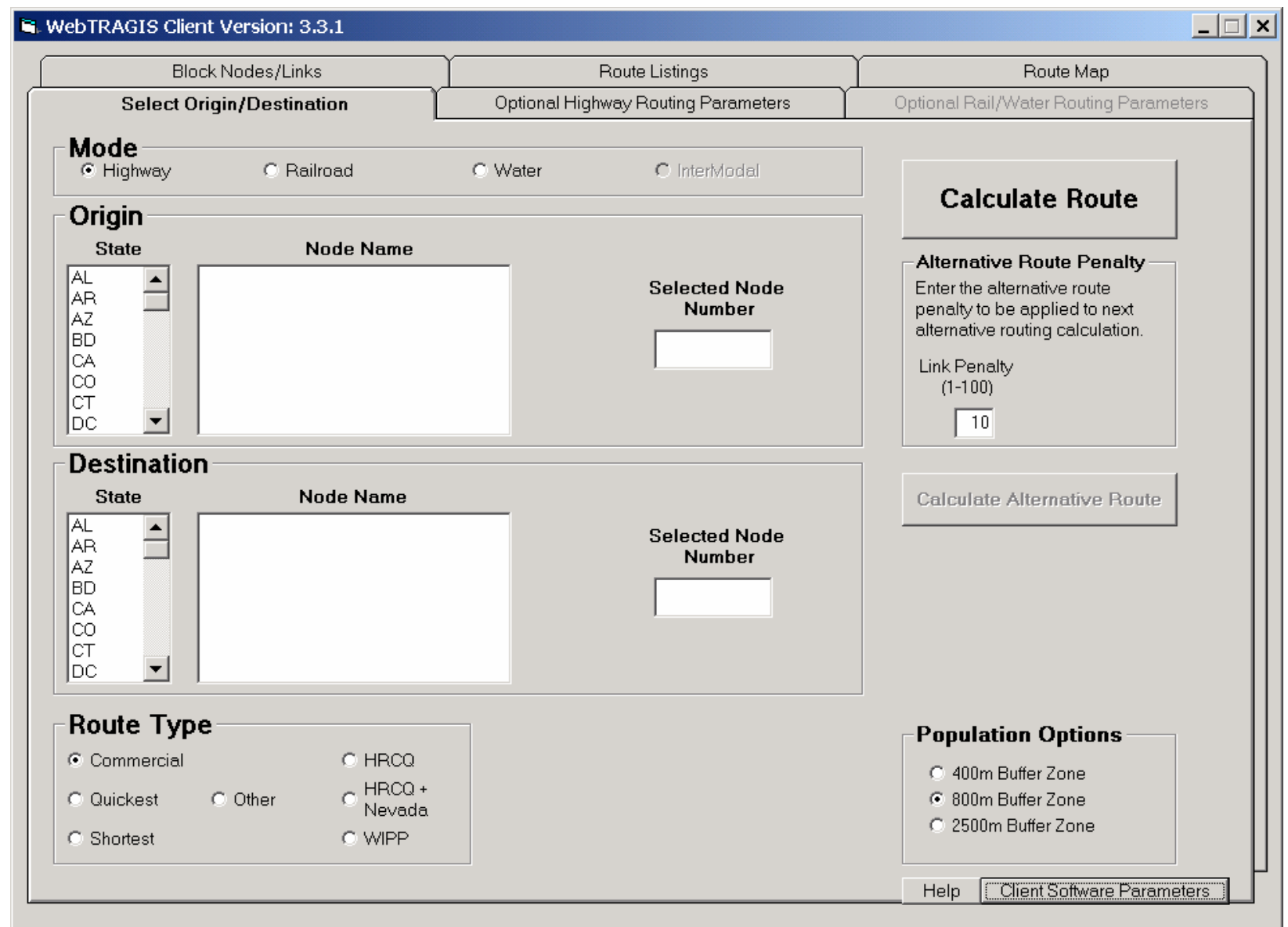

Fig. 3.2. Select Origin/Destination screen for highway routing.

\subsubsection{Alternative method-manual entry of node number}

An alternative method is to enter the specific node number of an origin or destination. Using this method, the node number is typed directly into the Selected Node Number box. An example for the use this method is if a node at the edge of an Indian reservation is required as the start or end point of a route. The 
highway network has nodes inserted where roads cross tribal boundaries. These nodes have a name of "IRB" for Indian reservation boundary. Since these nodes are not uniquely named, they cannot be selected using the primary method.

Figure 3.3 illustrates an example along Interstate 40 in northeastern Arizona. The "Holbrook N I40 X246" node is identified near the western edge of the map. Navajo Nation is shown with the green shading. The first node to the northeast of the Holbrook node is identified as node number 41100256. All of the nodes along I-40 crossing at the edge of the Navajo Nation have a node name of "IRB" and cannot be selected from the scroll lists.

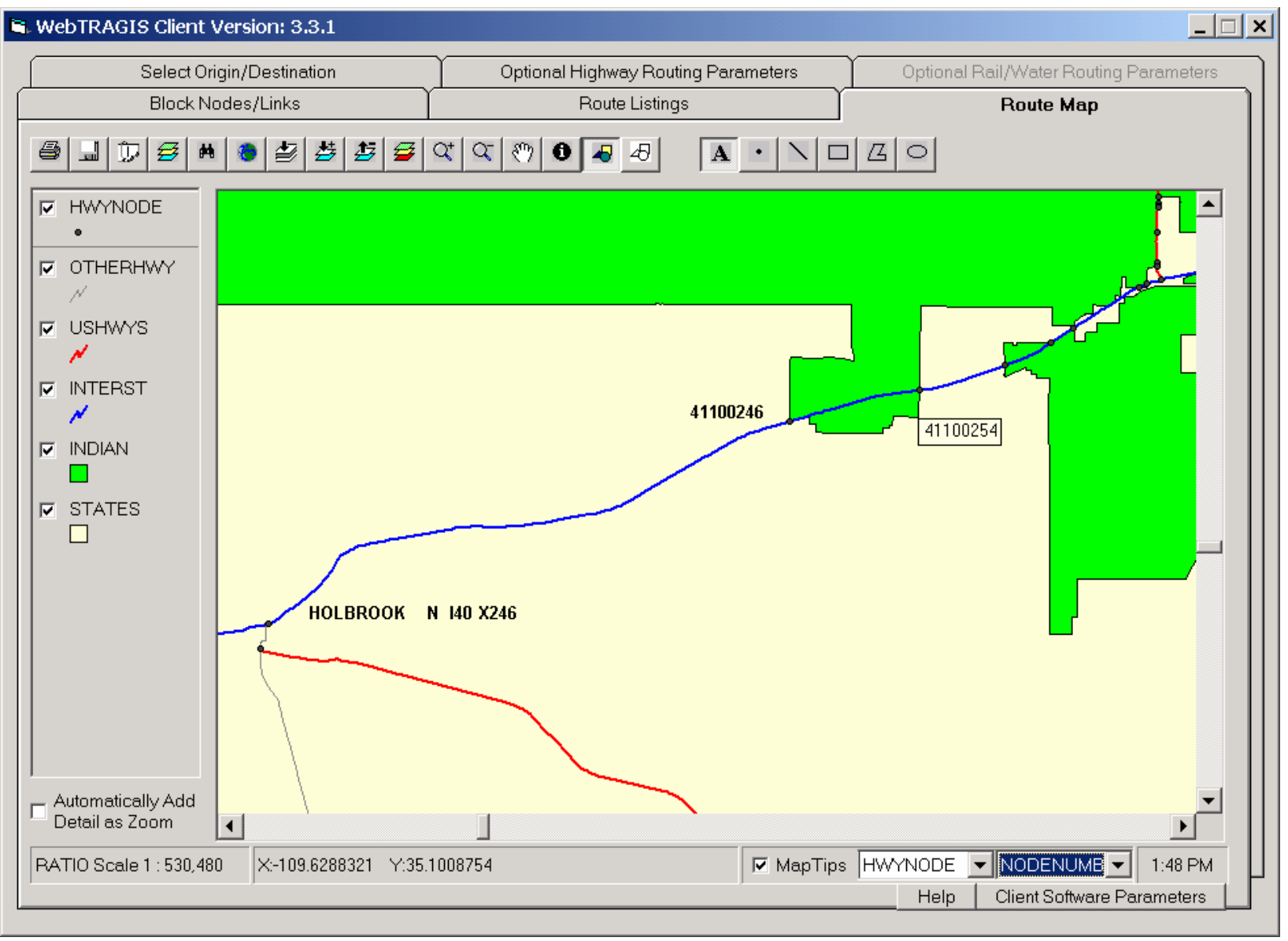

Fig. 3.3. Example of determining a highway node number from the Route Map screen.

To determine a node number, click on the Route Map screen tab and then activate the MapTips function at the bottom edge of the window. The pull down box to the right of the MapTips should identify the Hwynode map layer. If another map layer is identified, click on the adjacent down arrow and select Hwynode. The rightmost pull down box identifies the attribute field of the selected map layer. To determine a highway node number, select the Nodenumb attribute from the pull down list. Next zoom into the area of interest within the map window and move the pointer over a node and a small window will appear with the node number. In Fig. 3.3, the pointer is located over the second node to the northeast of the "Holbrook N I40 X246" node and is identified as node number 41100254. To use this node as either an origin or destination, go to the Select Origin/Destination screen and type this node number in the appropriate Selected Node Number box. 


\subsubsection{Route Type}

The Route Type selection box is located in the lower left portion of the Select Origin/Destination screen, as shown in Fig. 3.2. This sets the type of highway route that the model will calculate. The choices fall into two general classes. The basic routing options include the Commercial (default), Quickest, Shortest, and Other route types. Advance routing options include HRCQ, Nevada+HRCQ, and WIPP route types. Click the appropriate choice to select the proper route type.

\subsubsection{Basic routing options}

Routes are calculated by minimizing the total impedance between the origin and destination. The basic route types define impedance as a function of distance and driving time for each segment along a route that minimizes these factors as shown in Eq. (1):

$$
L=\operatorname{Min} \sum_{i}\left(\alpha D_{i}+\beta T_{i}\right)
$$

where

$\mathrm{L}=$ total impedance of a route;

$\alpha=$ distance bias;

$\mathrm{D}_{\mathrm{i}}=$ distance of segment $\mathrm{i}$, miles;

$\beta=$ time bias;

$\mathrm{T}_{\mathrm{i}}=$ time required to travel along segment $\mathrm{i}$, minutes.

Setting the values of the distance and time biases defines a particular routing criterion. A number of different types of routes can be predicted. The most commonly used route type is the Commercial route type, where $\alpha$ and $\beta$ are defined to be 0.3 and 0.7 respectfully This criterion is used to generate TRAGISpredicted routes that were verified through comparison with actual trucking routes used for a number of non-radioactive DOE shipments. Setting $\alpha=0.0$ and $\beta=1.0$, the most rapid route will be estimated (giving the entire weight to time). This is the setting for the Quickest route type. When $\alpha=1.0$ and $\beta=$ 0.0 , the shortest possible route will be calculated (giving the entire weight to distance). This is the setting for the Shortest route type. The Other route type is provided to allow for additional variations to the weighting of distance and time. When the Other type is selected an additional box appears in the lower center of the Select Origin/Destination screen, as shown in Fig 3.4. This box allows the user to select a ratio between the mile and time bias. To simplify entry, the two numbers are entered as whole numbers that need to sum to 100. Thus on the example in Fig 3.4, an even split between the mile and time bias would be entered as two values of 50 in the respective entry boxes. 


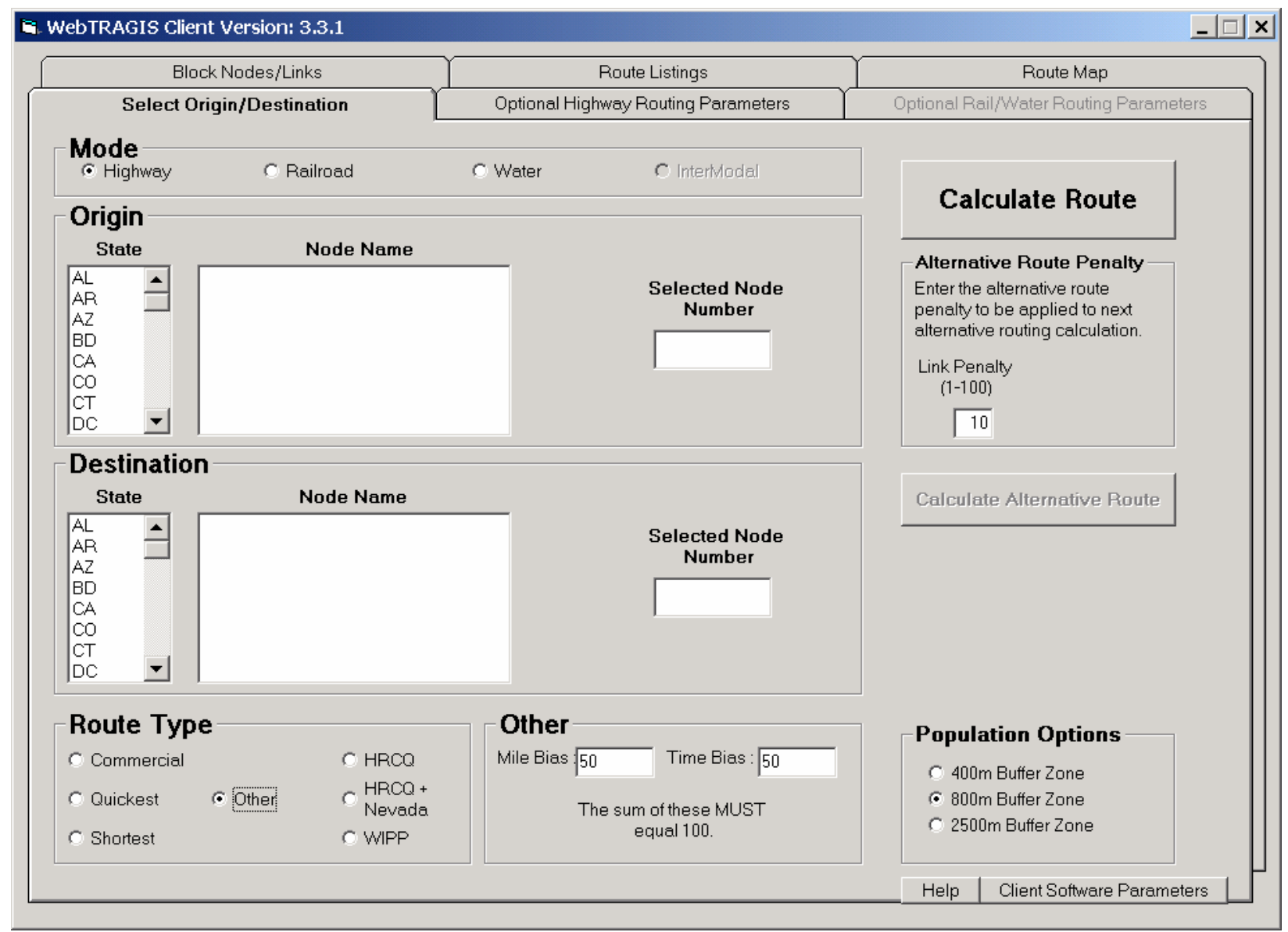

Fig. 3.4. Other route type setting box on highway Select Origin/Destination tab screen.

\subsubsection{Advanced routing options}

The additional route types, HRCQ, HRCQ+Nevada, and WIPP, are all based on DOT routing regulations (49 CFR 397.101) in the Code of Federal Regulations (CFR) for the shipment of highway route-controlled quantities (HRCQ) of radioactive materials. The HRCQ option follows the DOT routing requirements. The HRCQ+Nevada and WIPP follow the same DOT requirements but enable state specified routing options for shipments to Yucca Mountain and WIPP, respectively. The DOT regulations state that HRCQ shipments shall operate only over preferred routes, which primarily follow Interstate System highways, Interstate System bypass or beltway around a city, and state designated preferred routes. State routing agencies may designate preferred routes as an alternative to, or in addition to, one or more Interstate highways. In making this determination, the state must show that the alternative preferred route is as safe as the Interstate route that it is replacing and must register all such designated preferred routes with DOT. The TRAGIS highway network includes the designations of preferred routes and statedesignated routes for HRCQ shipments. Routes are to be selected that reduce time in transit over the preferred route segment of the trip. Frequently the origin and destination of HRCQ shipments are not located on preferred routes. The DOT routing requirements require that the shortest distance route between the pickup location to the nearest preferred route entry location and the shortest distance route to the destination from the nearest preferred route exit location be used. All of these requirements are incorporated into the HRCQ route type. The impedance equation for calculating a HRCQ route is shown in Eq. (2): 


$$
L=\operatorname{Min} \sum_{i}\left(\alpha D_{i}+\beta T_{i}\right) \gamma
$$

where

$$
\begin{aligned}
& \mathrm{L}=\text { total impedance of a route; } \\
& \alpha=\text { distance bias, with } \\
& \alpha=0.0 \text { if preferred route, } \\
& \alpha=1.0 \text { if non-preferred route; } \\
& \mathrm{D}_{\mathrm{i}}=\text { distance of segment } \mathrm{i} \text {, miles; } \\
& \beta=\text { time bias, with } \\
& \beta=1.0 \text { if preferred route, } \\
& \beta=0.0 \text { if non-preferred route; } \\
& \mathrm{T}_{\mathrm{i}}=\text { time required to travel along segment } \mathrm{i} \text {, minutes; } \\
& \gamma=\text { non-preferred route multiplier, with } \\
& \gamma=1.0 \text { if preferred route, } \\
& \gamma=30.0 \text { if non-preferred route. }
\end{aligned}
$$

The HRCQ, HRCQ+Nevada, and WIPP route types calculate the quickest route over preferred roads and a penalized shortest route over non-preferred roads. This penalization is the purpose of the nonpreferred route multiplier. The default value of the non-preferred route multiplier $(\gamma)$ has been determined empirically and ensures that the calculated route will conform to DOT regulations. When one of the preferred route types is selected a Preferred Route box appears on the right side of the Select Origin/Destination tab window to allow modification of the non-preferred route multiplier, as shown in Fig. 3.5. It is recommended that the value of the non-preferred route multiplier not be changed. If this value is lowered, the model may generate routes that do not conform to DOT regulations. The ability to adjust this parameter is provided to allow users the ability to test the sensitivity of different values on the preferred route types.

The HRCQ+Nevada route type is a modification of the HRCQ route type that allows analysis of six different access routes that were evaluated in the Final Environmental Impact Statement for a Geologic Repository for the Disposal of Spent Nuclear Fuel and High-Level Radioactive Waste at Yucca Mountain, Nye County, Nevada (DOE 2002). Two routes (A and B) approach Yucca Mountain from the north and four routes (C through F) approach Yucca Mountain from California, as shown in Fig. 3.6.

- Route A begins at I-80 in Wendover and follows US 93A, US 93, US 6, State 318, State 375, US 93, I-15, State 215, and US 95 (via Ely, Hiko, and Las Vegas) to Yucca Mountain.

- Route B also begins at I-80 in Wendover and follows US 93A, US 93, US 6, and US 95 (via Ely, Tonopah, and Amargosa Valley) to Yucca Mountain.

- Route C begins at I-15 in Baker, California, and follows State 127, State 373, and US 95 (via Amargosa Valley) to Yucca Mountain.

- Route D also begins at Baker, California, and follows I-15, State 160, and US 95 (via Arden and Pahrump) to Yucca Mountain.

- Route E begins at I-40 near Needles, California, and follows US 95, State 164, I-15, State 127, and US 95 (via Searchlight, Baker, and Amargosa Valley) to Yucca Mountain.

- Route F also begins at I-40 near Needles, California, and follows US 95, State 164, I-15, State 160, and US 95 (via Searchlight, Arden, and Pahrump) to Yucca Mountain. 


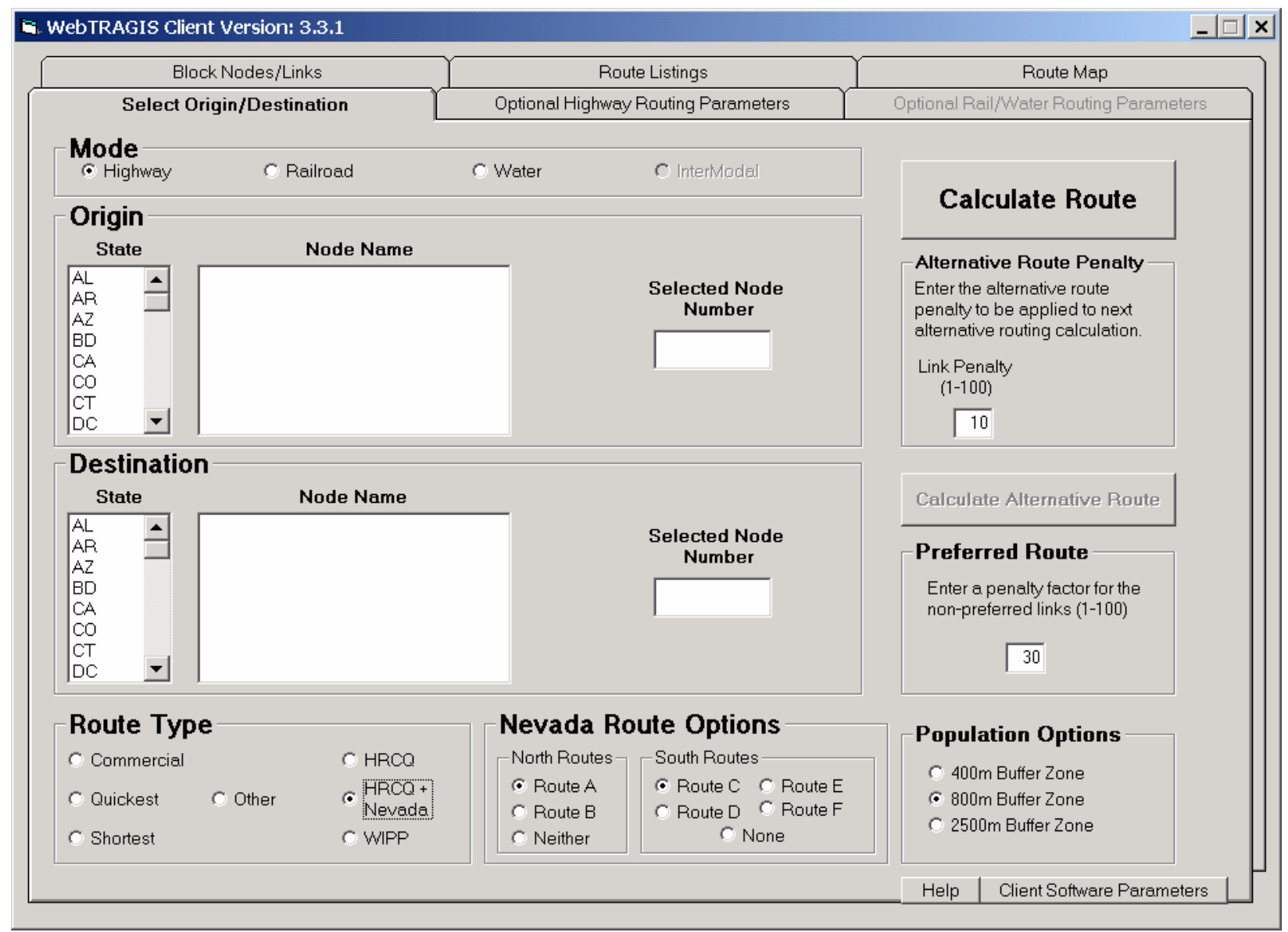

Fig. 3.5. Nevada Route Options box in the highway Select Origin/Destination tab screen.

When the Nevada+HRCQ route type is activated a Nevada Route Options box appears in the lower center of the Select Origin/Destination screen, as shown in Fig. 3.5. This option is designed to allow access to Yucca Mountain by only one of the Nevada route options or one north and one south access route. The reasoning for providing access from both directions is to allow the model to determine the appropriate access for a specific origin. For example, a route originating in Idaho would use a northern access while a route from Arizona would use a southern access. By default, both Route A and Route C are selected. If only one northern access is desired, select the None option in the South Routes box.

Conversely, if only one southern access is needed, select the Neither option in the North Routes box. The selection of both Neither and None essentially changes the Route Type back to a standard HRCQ route.

The WIPP route type is another preferred routing option and a modification of HRCQ coupled with New Mexico designated routes plus other state-specified WIPP preferred routes. This route type should only be used to calculate routes to the Waste Isolation Pilot Plant near Carlsbad, New Mexico. The State of New Mexico has designated a number of highways that are to be used to access the WIPP facility.

Additionally, DOE has agreements with Texas on the use non-Interstate highways for WIPP preferred routes. The WIPP route type activates these preferred roads. These designations are only valid for WIPP shipments and are not valid for other HRCQ shipments. It should be noted that DOE takes other factors into consideration on deciding specific WIPP routes. For this reason, WebTRAGIS may not replicate the planned routes from some of the sites to WIPP. Please consult the WIPP web site at http://www.wipp.carlsbad.nm.us/routes.htm for a map showing the current planned transportation routes. 
Use of features such as State Blocking, discussed in Sect. 3.6.3, can assist in replicating the routes shown on the WIPP web site.

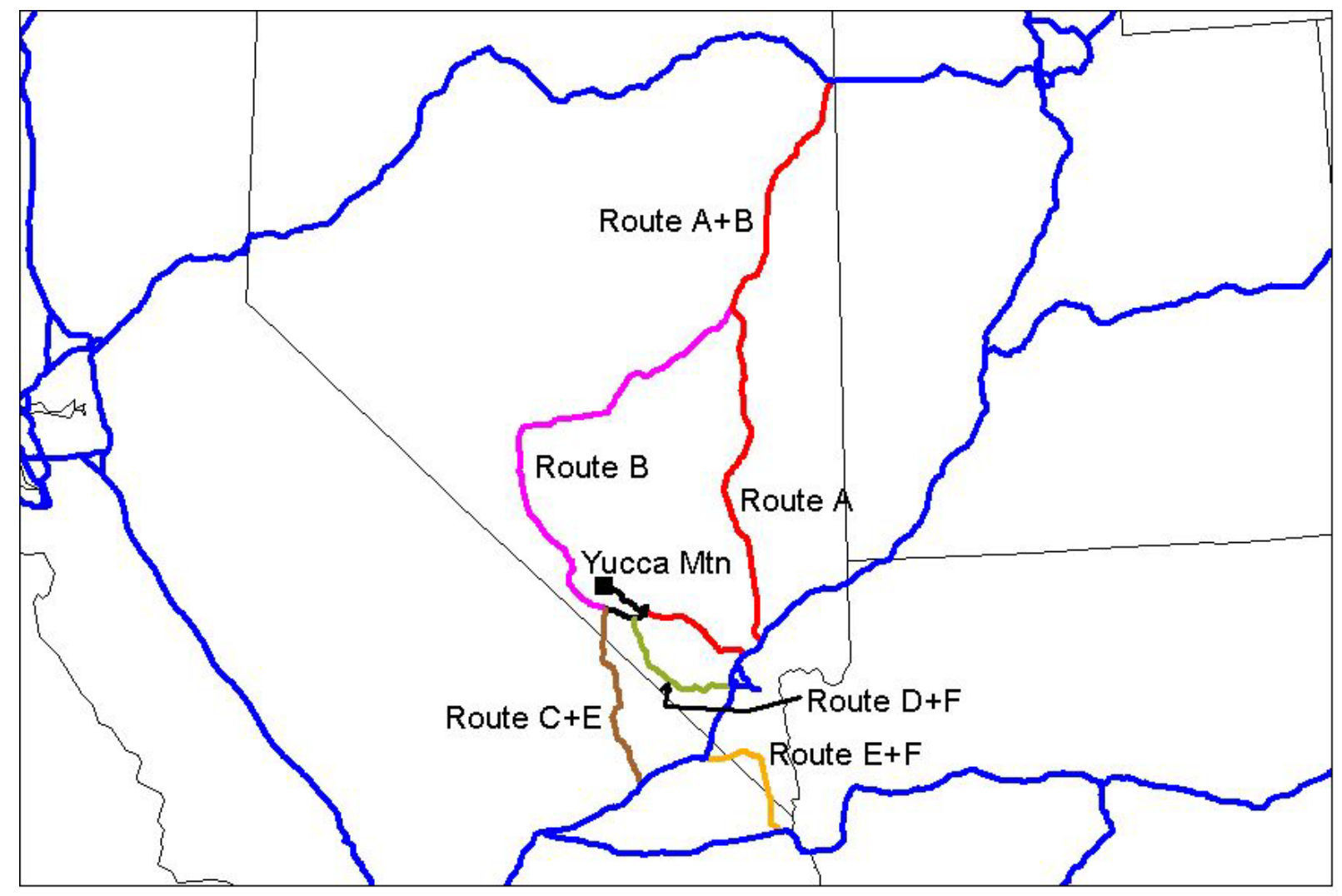

Fig. 3.6. Nevada highway route options.

\subsubsection{Population Options}

In addition to route selection, one of the most important applications of TRAGIS is to provide population density and count data along routes. The population data are TRAGIS is derived from the LandScan USA 15-arc second (approximately $360 \mathrm{~m}$ by $460 \mathrm{~m}$ ) grid cell population database (Bhaduri 2002). This database represents nighttime population distribution and is developed from a combination of data sources including 2000 U.S. Census Bureau block group population, roads from the Census TIGER data, slope from the NIMA Digital Terrain Elevation Data, and land cover from the USGS National Land Cover Database. The population data are based on geographic buffers constructed around the transportation route. Data on three different buffer distances are available in TRAGIS $-400,800$ and 2500 meters either side of the route. By default, the model selects the 800 meter-buffer width. To examine the population within one of the other corridor distances, select the appropriate distance within the Population Options box in the lower right portion of the Select Origin/Destination screen. It needs be noted that the selection of a different buffer distance will not be executed until the Calculate Route button is pressed. The population along a route is displayed using the Population Data Listing button on the Route Listings screen. (See Sect. 3.4.8 for a description of the output data.) For a discussion of routespecific population density, refer back to Sect. 2.5, TRAGIS Poulation Data. 


\subsubsection{Calculate Route}

After an origin, destination, route type and other options have been selected, click the Calculate Route button on the upper right side portion of the screen. If either an origin or destination is not selected, an error message box will appear with the message that an origin and destination need to be selected before a route can be calculated. WebTRAGIS will take a few seconds to calculate the highway route and then display the Route Listings window showing the standard listing for the route. Section 3.4 provides explanation of the route listings.

\subsubsection{Calculate Alternative Route}

After a route has been calculated, the Calculate Alternative Route button becomes active allowing the user to generate alternative routes. This feature is useful to determine if additional HRCQ routes exist that are similar, but have slightly higher impedance values than the optimal route selected by WebTRAGIS. In preparation for running an alternative route, all links comprising the current route are penalized by the Alternative Route Penalty factor. The default value for the Alternative Route Penalty is 10 . This adds an additional 10 percent to the existing impedance of each previously used link. Due to the increased impedances, alternative routes will tend to use a different set of links. When running multiple alternative routes, the penalization of multiple previously used links is multiplicative. Entering a different value in the Alternative Route Penalty box, located between the Calculate Route and Calculate Alternative Route buttons, can change this factor. It is suggested that care be taken in adjusting Alternative Route Penalty.

To calculate an alternative route, go back to the Select Origin/Destination screen and press the Calculate Alternative Route button. Depending upon numerous factors, several alternative routes can be calculated with this feature. The number of viable alternative routes that TRAGIS can calculate is dependent upon the density of the network between the origin and destination. A very sparse network may not provide any other path. In that case, the model will display the same route. (No message is provided by WebTRAGIS indicating that an identical route was calculated.) In other cases, where there is more connectivity with the network, the model will provide several alternative routes. However, repeated attempts to calculate numerous alternative routes may begin to lead to nonsensical results. If the results are unsatisfactory, a better approach for "alternative" routes is to block certain links, nodes, or even states from consideration. Read Sect. 3.6 for additional details

\subsection{ROUTE LISTINGS SCREEN}

The Route Listings screen provides access to the text-based output from WebTRAGIS as well as access to files for debugging purposes. When the model generates a route, the program initially displays the Route Listings screen with the standard listing of the calculated route, as shown in Fig. 3.7. A number of buttons are located along the upper edge of the screen. The function of each of these buttons is discussed below.

The main portion of the route listing provides a description of route. The route listing has a legend with the following column headings-Miles, Hwy Sign, City, Dir, Junction, State, Dist, Time, Date, Hour, and (only on the detailed listing) Impedance. A discussion of the route description data follows:

- Miles-The first column is the mileage from the previous named place to the current named place. Thus the first entry will also be zero. In Fig. 3.6, the distance from DOE Germantown to the entrance of $\mathrm{I} 270$ at Exit 15 is 0.3 miles.

- Hwy Sign-The second column identifies the highway route used between the previous and current line. If the highway has multiple numbers, up to two will be listed. In the example in 
Fig. 3.6, no multiple signed highways are encountered. Between the Germantown NE I270, X15 node and the Garrett Park S I270, I495 node, the route travels 14.7 miles on I270.

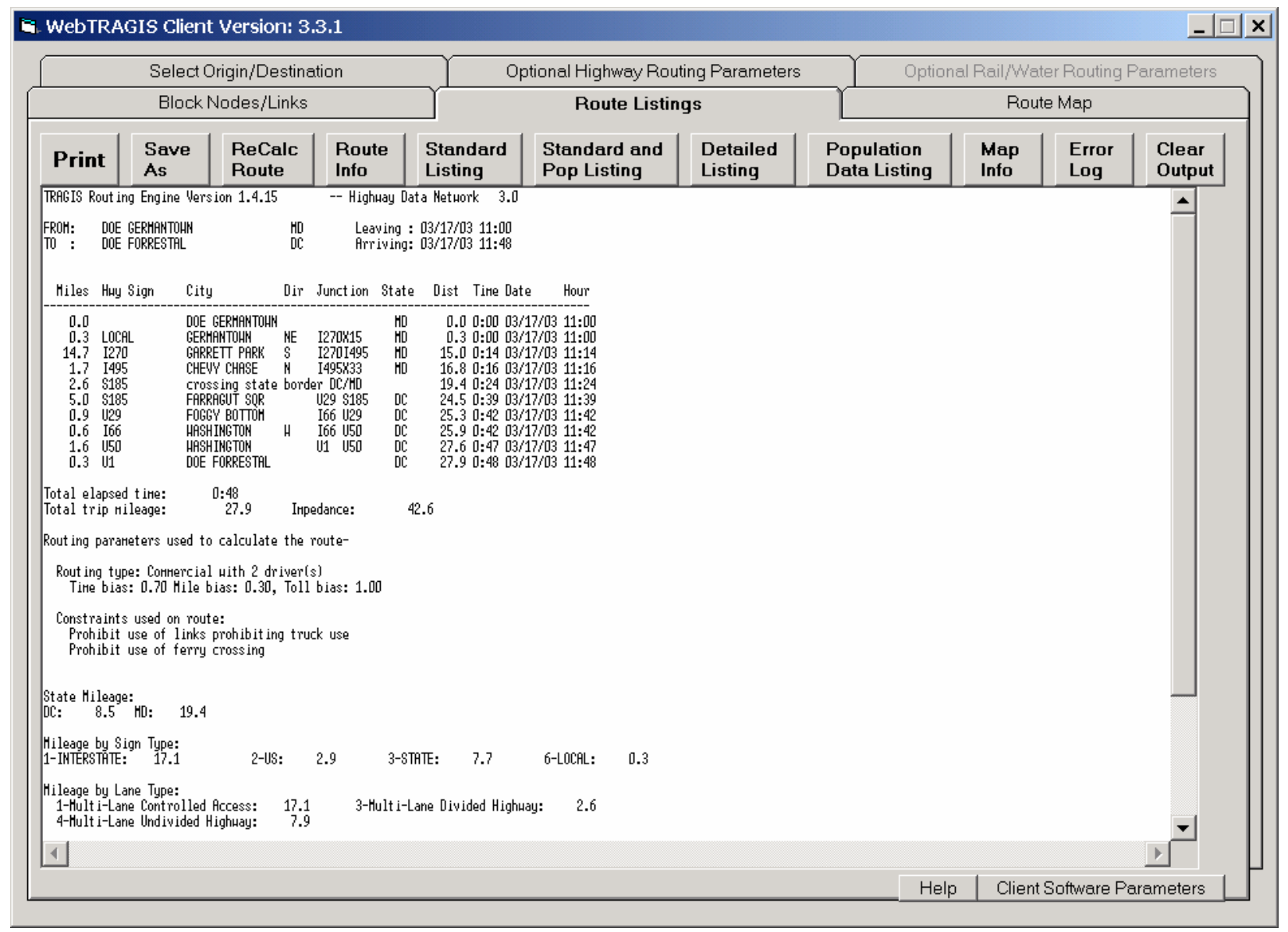

Fig. 3.7. Standard highway route listing.

- City-The third column contains the city portion of the node name. In this example, the route originates at the DOE Germantown node. The route listing also inserts special text in the city column indicating rest breaks, state inspections, and state border crossings. This example includes a line indicating the state border crossing between Maryland and the District of Columbia.

- Dir-The direction portion of the node name is the direction the node is in relation to the center of the city or community that the node is named after. This direction modifier does not refer to the direction that the route is traveling. The second node on the route listing, Germantown NE I270X15, is located northeast of the center of Germantown, Maryland.

- Junction-The junction portion of the node name assists in providing more information as to the location the node. In Fig. 3.6, The Chevy Chase node is located at Exit 33 on I495.

- State-This column identifies the state in which the node is located.

- Dist-The distance column provides the cumulative mileage from the start of the route. For the line indicating the "crossing state border DC/MD," the cumulative mileage for the route is 19.4 miles. Please note that the route listing only reports mileage to the nearest tenth of a mile. The TRAGIS highway network stores the mileage for each link to the nearest thousandths of a mile. This can introduce a rounding error. Note that the cumulative distance for the route at Chevy Chase is 16.8 miles, but if you sum the individual link mileage in the first column up through the 
Chevy Chase node, you obtain only 16.7 miles. So take care when trying to verify some numbers reported by the model.

- Time-The time column provides an elapsed time from the start of the trip. The first line in the route listing will always indicate 0:00. In this example, the elapsed time between DOE Germantown and the Farragut Square node is 39 minutes.

- Date-WebTRAGIS allows the use of either the current computer system date or an actual date specified by the user for the start of a shipment. (See Sect. 3.5.3 for instructions on setting a start date.) This column of the route description provides the date when the shipment passes through the node name listed on the line. For cross-country routes this can become important due to the length of the trip.

- Hour - WebTRAGIS allows the user to either use the current computer system time or to specify an actual time for the start of a shipment. (See Sect. 3.5.3 for instructions to set a specified start time.) This column reports the expected time a shipment would pass through the node name listed earlier on the line. The TRAGIS highway network includes data on time zones and this field will reflect adjustments when crossing time zone boundaries.

- Impedance-The impedance column is only provided on the detailed route listing. This column reports the calculated impedance for each link. The formulation of this value varies with the Route Type used to calculate the route (see discussion in Sect. 3.3.2). This is an advanced feature that most users will not need to be concerned about.

\subsubsection{Print}

The Print button sends the information listed in the Route Listings screen to the printer connected to the PC running the client version of WebTRAGIS. The default windows printer and settings are used to print data displayed on the screen.

\subsubsection{Save As}

The Save As button provides a method of writing the information listed in the Route Listings screen to a standard text file. The function of this button is the same for whatever previous button is used to display data on this screen, be it the Detailed Listing, Population Data Listing, Route Info, or Standard Listing button. When this button is pressed a Save As window is opened, which allows the user to select the appropriate drive, directory, and file name where the text information from the screen will be saved. By default, this function saves all files with the ".txt" extension.

\subsubsection{ReCalc Route}

The ReCalc Route button opens a window to select a previously saved Route Info file. After this file is selected, WebTRAGIS will recalculate the route based on the parameters in the Route Info file. The normal user will rarely, if ever, need to use this feature. This feature is primarily used to assist users who are having problems with the model and allows TRAGIS development staff to be able to reproduce user problems.

\subsubsection{Route Info}

The Route Info button displays the most recent set of parameters used to create a route (such as the origin, destination, option settings, blocked nodes and links, etc.). This set of parameters is sent over the Internet to the TRAGIS server to calculate the route. If the user experiences any problems with generating a particular route, this button should be used to examine the parameter settings. If further assistance is 
required, the Route Info data should be saved in a file, using the Save As button, and then the saved file can be e-mailed to the TRAGIS development staff for debugging.

\subsubsection{Standard Listing}

The Standard Listing button displays the route description of the most recent route calculated. (WebTRAGIS displays this listing following the calculation of each route.) Figure 3.7 provides an example of the standard listing of a route between the DOE Germantown offices in Maryland, and the DOE Forrestal offices in the District of Columbia.

The first line of the output provides version of the TRAGIS route engine and highway data network used to generate the route. This information is extremely useful when comparing model runs over time. Modifications to either the routing engine or network database could slightly change results. The next two lines identify the origin and destination of the route, along with the departure and arrival date and time. This is followed by a description of the route. The first column is the distance, in miles, between each location identified in the listing. The next column is the identification of the road used on this segment. (See Sect. 3.2 for the nomenclature used for highway and node names.) The route listing will provide either one or two road identifications for each segment (e.g., a dual-designation road such as I-40/I-75). Next the name of the node is listed. Node names generally are comprised of a town or city name, a directional modifier indicating the direction that the node is from the center of the town, and junction information. In the example shown in Fig. 3.7, the penultimate node of the route is the intersection of U.S. 1 and U.S. 50 in Washington, DC. In some cases, actual facilities are identified, such as DOE Germantown. The last portion of the node name is the postal abbreviation of the state where this node is located. The next two columns identify the cumulative mileage and driving time. Finally, the last two columns identify the date and time when the shipment is expected to pass the node. By default, the model assumes the departure time to be the current time from the computer. The user can set the departure date and time on the Optional Highway Routing Parameters screen, which is discussed in Sect. 3.5.3.

Some users have difficulty in interpreting the route listing. The following discussion should explain how to understand this information. In the example shown in Fig. 3.7, the route originates at the DOE Germantown facility at 11:00 a.m. The first portion of the route travels 0.3 miles on a local road and turns onto I-270 at Exit 15. This location is northeast of Germantown, Maryland. After traveling 14.7 miles on I-270, the shipment exits to I-495, south of Garrett Park, and then travels 1.7 miles on I495 to exit 33, north of Chevy Chase, Maryland. At this location, the length of the route is 16.8 miles, and the trip has taken about 16 minutes. The route next travels on State 185 for 2.6 miles where the route crosses the state line between Maryland and the District of Columbia. At this point, the cumulative distance of the route is 19.4 miles and has taken 24 minutes. The route continues on State 185 (DC does not number these roads, but because this road continues into the District, the TRAGIS highway network uses Maryland state highway numbers) to Farragut Square, at the junction of U.S. 29 and State 185. The route follows U.S. 29 for 0.9 miles to the junction with I66 at Foggy Bottom. Next, I66 is followed for 0.6 miles to the junction of I66 and U.S. 50 and then U.S. 50 is used for 1.6 miles to the junction of U.S. 1 and 50. The final line of the route shows that U.S. 1 is used for 0.3 miles to reach the DOE Forrestal site, the total distance of the route is 27.9 miles, and the elapsed time is 48 minutes, with an arrival time of 12:14 p.m. It should be noted that the time and distance might vary slightly over the route due to rounding effects.

Following the route description, a summary of the total elapsed time, trip mileage, and impedance is listed. Impedance is the mathematical value of the route (see discussion in Sect. 3.3.2) and is useful when comparing two similar routes with the same route type. This is followed by a summary of the routing parameters and constraints used to calculate the route. A summary of mileage by state, highway sign type, and lane type is also provided. Finally, a summary of mileage with Native American Tribal Land and a 
breakdown of mileage within counties in Nevada are provided. (Some of this information extends beyond the edge of the screen shown in Fig. 3.7.)

\subsubsection{Standard and Pop Listing}

The Standard and Pop Listing button displays a combination of the information shown with both the Standard Listing (see Sect. 3.4.5) and Population Data Listing (see Sect. 3.4.8) buttons.

\subsubsection{Detailed Listing}

The Detailed Listing button displays a listing that includes all named nodes along a route. The Standard Listing only show nodes when road designations change. Figure 3.8 displays the same route shown in Fig. 3.7. With this relatively short route, the detailed listing is only several lines longer. For long distance routes, a detailed listing can be quite lengthy. The most extreme case would be to compare a route along a single cross-country road. The detailed listing provides one additional column of information - the impedance value for each link. This feature is useful when using various constraints to determine the links with high impedance values.

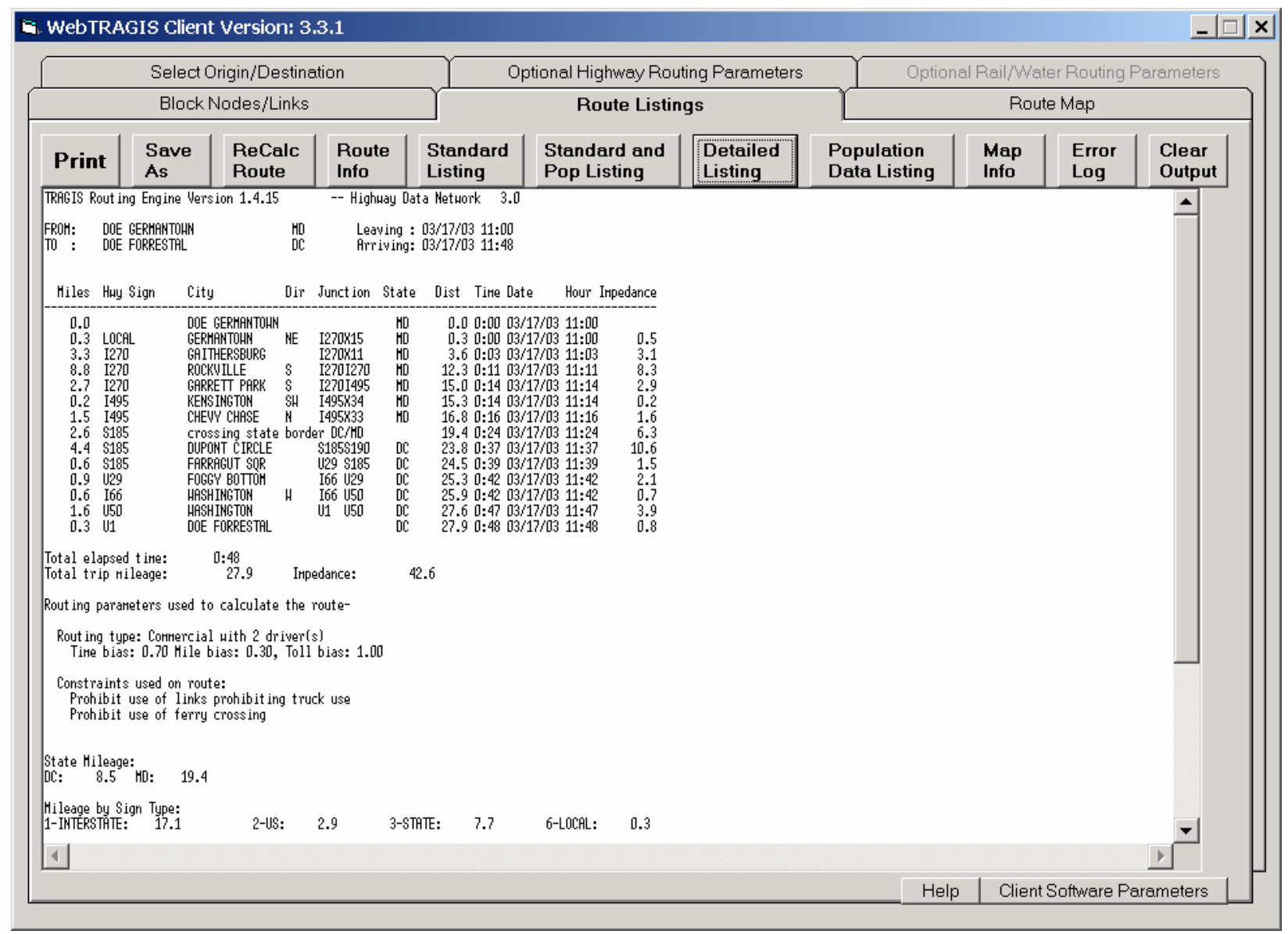

Fig. 3.8. Detailed highway route listings. 


\subsubsection{Population Data Listing}

The Population Data Listing button displays a population density table, RADTRAN input data, and the population counts within the selected route buffer for the most recent calculated route. With the 2000 Census information, the population data in TRAGIS is calculated based on a buffer distance from the route. The buffer distance needs to be established prior to the calculation of the route. Selecting a different distance on the Select Origin/Destination screen and then switching to the Route Listings screen without pressing the Calculate Route button will not change the data in the population data listing screen. However, if a different buffer zone distance is selected and the Calculate Route button is pressed on the Select Origin/Destination screen, the Population Data Listing will display the updated population data.

Figure 3.9 shows the population data listing for the route between DOE Germantown, Maryland, and DOE Forrestal in the District of Columbia. The listing provides the TRAGIS routing engine and highway network version numbers, the buffer zone distance, and the origin and destination of the route in the upper portion of the listing. The population density table includes 11 population-density categories ranging from 0 to over 9996 people per square mile. Entries in the table show the distance of the route by state within each category. At the end of the table, total mileage and percentage summary information is provided for the route. Next this information is summarized from the 11 categories into rural, suburban, and urban groupings with data calculated into a weighted population-density value and distance for each of these three groups. This information is useful as route specific input for risk analysis using the RADTRAN computer code (Neuhauser 2000) developed by Sandia National Laboratories or the RISKIND computer

Input data for the RADTRAN model requests population density and distance data for rural, suburban, and urban areas. The basis for these three categories is:

- Rural Categories below 139 people per square mile,

- Suburban Categories between 139 and 3326 people per square mile,

- Urban Categories above 3326 people per square mile.

The model reports the route specific weighted population density and distance data (in both English and metric units) for the three population categories.

With the improved calculation methods used to determine the population numbers, the user should note that these data are only estimates. Also, these numbers represent nighttime population figures and that daytime population distributions are significantly different. It is best to use these population figures for global comparisons of different routes rather than an absolute value.

\subsubsection{Map Info}

The Map Info button displays a text file with the end point coordinates of each individual link for the most recently calculated route. This data only provides node coordinates and does not provide the shape coordinates for the links. Information on this screen is used to draw the straight-line segments on the Route Map screen discussed in Sect 3.7.3.1. Most users will not have much use for viewing this data.

\subsubsection{Error Log}

If the model generates an error, it will automatically be displayed in the Route Listings screen. The Error Log button can be used to redisplay any error messages that the model generates on the server. If the error message does not provide sufficient assistance to remedy the problem, please contact one of the TRAGIS 


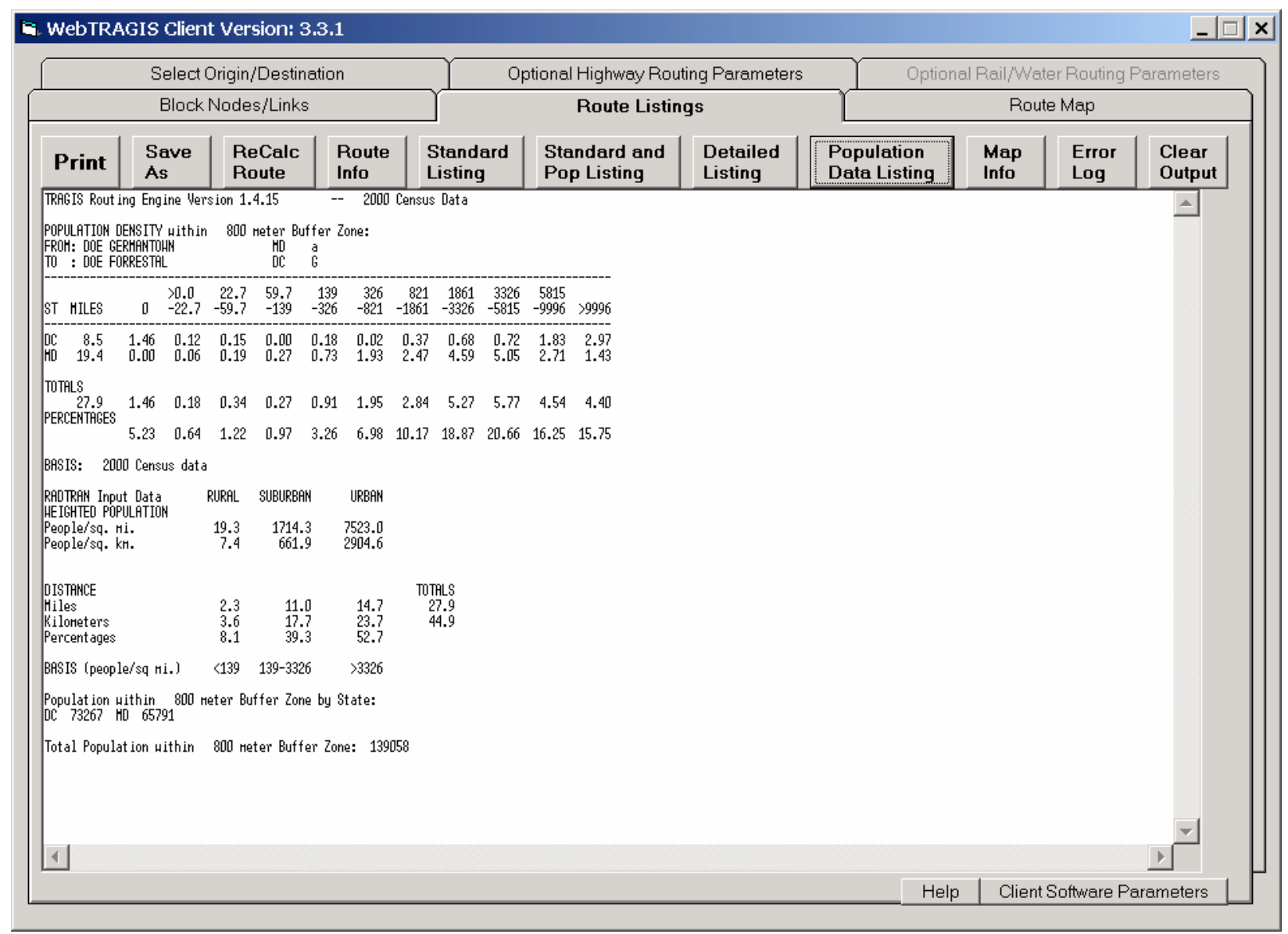

Fig. 3.9. Highway population data listing.

development staff regarding the error message. Telephone numbers for the TRAGIS development staff can be determined by clicking on the Help button on the lower edge of any WebTRAGIS screen.

\subsubsection{Clear Output}

The Clear Output button will remove the contents of all the WebTRAGIS output files (the route info, standard listing, detailed listing, population data listing, map info, and error log files) that can be viewed in the Route Listings screen. The output files for WebTRAGIS are downloaded from the server to the client computer following the calculation of each route and overwrite earlier copies. This button provides a means to delete the output files on the client computer.

\subsection{OPTIONAL HIGHWAY ROUTING PARAMETERS SCREEN}

The Optional Highway Routing Parameters screen tab provides access to a number of parameters that control various aspects of WebTRAGIS routing functions. Selecting the Optional Highway Routing Parameters tab will display a window similar to that shown in Fig. 3.10. This window identifies all the additional options available for highway routing. The various optional highway routing parameters are discussed below. 


\subsubsection{Driver Options}

The Driver Options parameters allow changes that effect the estimated driving time of the route determined by the model. Changes to some of these parameters do not effect the calculation of the path of the route but can change the duration of the shipment. By default, the model uses a two-driver team. To select the one driver option, either click on the One Driver text or the radio button preceding this text.

When the Optional Highway Routing Parameters screen is displayed, the additional parameters displayed in the Driver Options in Fig 3.10 are not shown. To display the various parameter settings, double click either the radio button or the text to the right of the radio buttons. The numbers initially displayed, and provided in the discussions below, are the default values. Editing the displayed values will change each of these parameters. By default, the model assumes that a two-driver team is used on a shipment and that a 30-minute rest will occur every four hours. If changes are made to any of these parameters, the model will list the new values on the route listing.

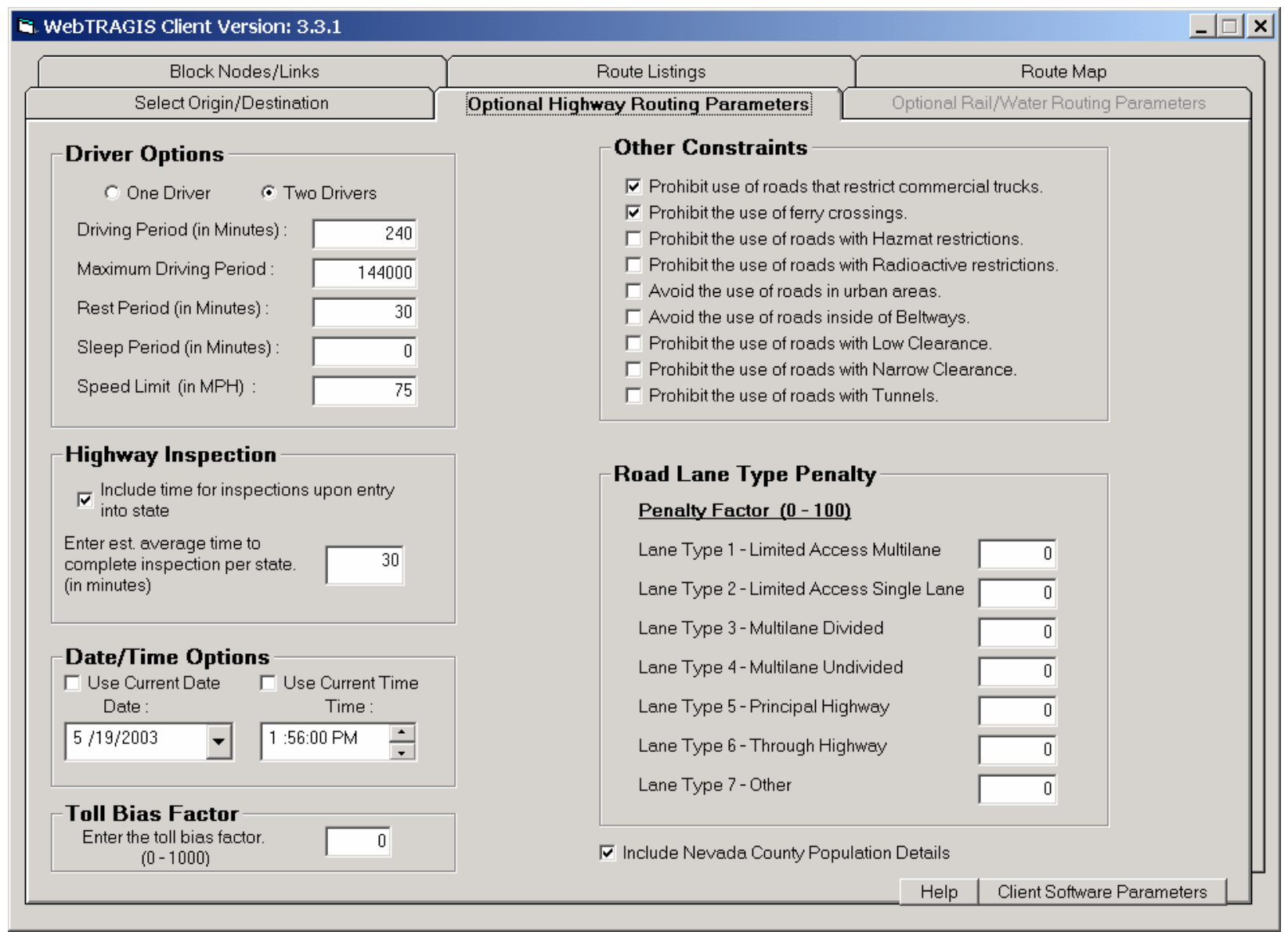

Fig. 3.10. Optional highway routing parameters.

\subsubsection{One driver}

The first four items in this option window involve the periods of time for driving and resting. With a One Driver shipment, the default pattern allows a driver to work for 5 hours (driving period) and then take a 0.5 -hour break (rest period). After this break, another 5 hours of driving can occur, for a total of 10 hours (maximum driving period), before an 8-hour rest period (sleep period) is required. These time parameters 
are entered as minutes and can be changed to meet the specific needs of a particular study. It is important that the maximum driving period should always be twice the value of the driving period.

\subsubsection{Two drivers}

As with the one driver option, the first four items in this option window involve the periods of time for driving and resting. With a Two Drivers shipment, the default pattern allows the two-driver team to work for 4 hours (driving period) followed by a 0.5 -hour break (rest period). The default values for the maximum driving period and the sleep period are set to a large value and to zero, respectively, because with a two-driver team one member can rest while the other drives allowing around the clock operation.

\subsubsection{Maximum driving speed}

The speed limit parameter of the Driver Option permits a specification of the maximum speed that a shipment will travel. Congressional action in 1995 lifted national speed limits and allowed states to specify their own limits. For some shipments, there may be a need to specify an upper limit that the vehicle will not exceed. The speed limit parameter in WebTRAGIS provides such a capability. The default value for this parameter in WebTRAGIS is $75 \mathrm{mph}$. To specify a lower maximum travel speed, enter the new value in the speed limit box. However, WebTRAGIS will use the speed specified in the highway database for each highway link - except for those links with speeds higher will be assumed to be the value the user specified. Adjusting this value may affect the route calculation; it is also used to determine shipment duration and arrival time that are identified on the route listing.

\subsubsection{Highway Inspection}

WebTRAGIS provides an option to include a delay for state inspections of a shipment when a shipment crosses a state line. Figure 3.10 shows the Highway Inspection option selected along with a parameter box where the average inspection delay can be adjusted. The default value is 30 minutes.

This option will create a delay every time the calculated route crosses a state boundary to simulate the delay a shipment may experience if a state inspection is required. Use of this option will not change a route (e.g., such as minimizing the number of states traversed). It will only increase the overall travel time for the shipment. To activate the Highway Inspection option, click on this option to place a check mark in the small square. The user can specify the estimated delay, in minutes, for inspections. When this option is active, the route listing will identify the inspection delay on the route listing.

\subsubsection{Date/Time Options}

The Date/Time option allows the user to specify the departure date and time for a shipment. By default, TRAGIS uses the current date and time from the TRAGIS server computer. Because the TRAGIS server is located in the Eastern Time zone, the current time is based on this time zone. Due to the way the time function operates within the model, routes that originate in other time zones will have the starting time adjusted based on Eastern Time. For example, if it is noon in the Eastern Time zone, and the origin for a route is located in the Pacific Time zone, the model will indicate that the departure time is 9 a.m. (adjusted to the time zone for the origin of the route). The estimated time provided for each location on the route listing reflects the current time at each location, based on time zone boundaries.

If a specific departure date needs to be set, unselect (remove the check) the Use Current Date and the model will display the date field, as shown in Fig. 3.10. Next click on the down arrow on the right hand side of the date box to display a calendar of the specified month. Click on the left or right arrow buttons at 
the top of the calendar to move to the appropriate month and then click on the desired date. Alternatively, the user can highlight individual portions of the date field and manually edit the date.

To specify a departure time, unselect the Use Current Time and the model will display the time field, as shown in Fig. 3.10. Adjusting the time displayed within the time parameter box sets the starting time. Please note that TRAGIS interprets the entered time is as Eastern Time. So if a starting time of 8:00 a.m. Pacific Time is desired, the time should be entered as 11:00 a.m. The time function within TRAGIS automatically handles time changes between standard and daylight time.

\subsubsection{Toll Bias Factor}

The toll bias factor provides a method of placing an additional impedance value on the use of toll roads and bridges. By default, there is no penalty placed on toll facilities, as shown by the zero in the Toll Bias Factor box in Fig. 3.10. The value entered in the Toll Bias Factor is entered as a whole number and then the model divides this value by 100 and adds this result to 1 . So if a 15 percent toll bias were to be placed on toll facilities, the value of 15 would be entered in as the Toll Bias Factor. This would cause the existing impedance for all links with tolls being multiplied by a factor of 1.15 . An easy way to verify the effect of this feature is to use the detailed route listing to view individual link impedances before and then after applying this factor.

\subsubsection{Other Constraints}

The Other Constraints options are found in the upper right hand portion of the Optional Highway Routing Parameters screen, as shown in Fig. 3.10. Checking the box before each item activates the specific constraint. Two of the constraints are automatically activated by TRAGIS - roads that prohibit commercial truck traffic and use of ferry crossings. If there is a need to remove either of these constraints, uncheck the respective constraint. The TRAGIS highway network has these parameters defined as link attributes.

\subsubsection{Commercial trucks}

The commercial truck constraint prohibits the program from using any road that restricts commercial truck traffic. An example of such a prohibition is Interstate 66 inside the I-495 Capital Beltway in the Washington, DC, area; no commercial truck traffic is allowed on this road. Thus, for any routing of commercial traffic, this constraint needs to be active. In other instances, local governments have restricted commercial traffic from roads for through traffic. Examples of this include Atlanta, which bans through traffic from using highways within the beltway. Obviously traffic originating or terminating within the Atlanta can operate on those roads, but this option effectively restricts such traffic. In some instances, this option may need to be deactivated to access a node that may be in such an area. Another example of commercial truck prohibitions includes highways passing through national parks. By default, this constraint is activated at the start of a WebTRAGIS session. To deactivate this constraint, remove the check mark from this item by clicking on it.

Please note that this constraint is unrelated to the HRCQ routing type. Obviously, HRCQ radioactive shipments will not use these roads, but the commercial truck constraint applies to all commercial traffic. The HRCQ route type keeps shipments on preferred roads, which are primarily Interstate highways and by definition uses beltways around metropolitan areas.

\subsubsection{Ferry crossings}


Several ferry crossings are in the WebTRAGIS highway network between Long Island, New York, and Connecticut. The ferry-crossing constraint prohibits the use of a ferry on a route. By default, this constraint is activated at the start of a WebTRAGIS session. To deactivate this constraint, remove the check mark from this item by clicking on it.

\subsubsection{Hazardous material restrictions}

The WebTRAGIS highway network includes information for each link designating if a non-radioactive hazardous material restriction exists on that road segment. Information on such restrictions is based on data from the National Hazardous Material Route Registry, which is available at http://hazmat.fmcsa.dot.gov/. Hazardous material restrictions may exist for one or more hazard class, but a conservation approach is used in TRAGIS highway network. Thus, if any hazard class is prohibited, the link is designated as a hazmat restriction. Click on this item, placing a check mark on this constraint, to activate the hazardous materials restriction. An example of the hazmat restrictions include a number major highways inside the beltways of Cuyahoga and Lake counties in the Cleveland, Ohio, area.

\subsubsection{Radioactive material restrictions}

The WebTRAGIS highway network includes information for each link designating if radioactive material restriction exists on that road segment. Many state and local governments create separate restrictions for radioactive materials that are different from non-radioactive hazardous materials restrictions. Information on such restrictions is based on data from the National Hazardous Material Route Registry, which is available at http://hazmat.fmcsa.dot.gov/. To activate the radioactive materials restriction, click on this constraint to place a check mark on it. An example of radioactive material restriction is I-70 between Golden, Colorado, and the Utah state line.

\subsubsection{Avoid urban areas}

Roads in U.S. Census designated urbanized areas with populations exceeding 100,000 are identified in the TRAGIS highway database. The Census Bureau defines an urbanized area as a city and the adjacent densely settled surrounding area. For a large metropolitan area, the urbanized area includes the central city, suburbs, and other high-density areas adjacent to the incorporated places. Census reports provide maps of urbanized areas and any highway links that pass through an urbanized area exceeding 100,000 people is designated in the routing network for this constraint.

By activating the option to avoid roads in urban areas, the model multiplies the impedance of such links by 10,000 units. The effect is TRAGIS will attempt to avoid the use of roads within the designated urbanized areas. Routes may still pass through a designated urbanized area, but the model will select the minimal path through such areas. Nearly every link in the megalopolis region between Boston and Washington, DC, is included in this designation. Use of this constraint can create rather unusual routes and it should only be used sparingly.

\subsubsection{Avoid roads inside beltways}

The avoid use of roads inside of beltways constraint places a large penalty on the use of roads within Interstate beltways of larger metropolitan areas. This constraint is different from the commercial truck restriction in that this constraint blocks roads within all beltways whereas the other restriction only blocks these roads within metropolitan areas that have created such regulations. Also, this constraint is not related to the HRCQ-preferred routing option and does not need to be used with the HRCQ route type. It 
is included for examination of non-radioactive hazardous material routes where the user wishes to avoid roads within beltways.

Use of this constraint will cause the route to avoid going within the major beltways in large cities. If an origin or destination is selected within a beltway, the model will route to the location, but the minimum impedance path will be used. To activate this constraint, click on this item and place a check mark on it. All major cities with some type of interstate beltway will have roads within the beltway designated for this constraint.

\subsubsection{Low clearance restrictions}

Road segments with height clearances of $13 \mathrm{ft} 6$ in or less are identified in the TRAGIS highway network. An example is the Chesapeake Bay Bridge Tunnel, which has a height restriction of $13 \mathrm{ft} 6$ in. By activating this constraint, the model will prohibit the use of these road segments. Checking the box for this item will activate this constraint.

\subsubsection{Narrow clearance restrictions}

Road segments with width restrictions of $8 \mathrm{ft}$ or less are identified in the TRAGIS highway network. Permanent width restrictions are rather rare and temporary width restrictions are usually related to construction projects. By activating this constraint, the model will prohibit the use of these road segments. Checking the box for this item will activate this constraint.

\subsubsection{Tunnels}

The final constraint is a prohibition the use of roads with tunnels. Any road segment with a tunnel is identified by this constraint. This constraint is related only to the fact that a tunnel exists on the link and has no relationship to any type of hazardous material restrictions. If a tunnel has a hazardous material restriction, it would be identified with the hazardous materials constraint. To activate this constraint, click on the item to place a check mark on it.

\subsubsection{Road Lane Type Penalty}

The highway network has an attribute for each link indicating the road's lane configuration. The lane types used in the TRAGIS highway network include limited-access multilane, limited-access single lane, multilane divided, multilane undivided, principal highway, through highway, and other. The road lane type penalty feature allows users to weight each road segment based upon these seven classifications. A penalty factor ranging from 0 to 100 can be entered for each lane classification type. A factor of 0 places no additional penalty on a link while a factor of 100 doubles the impedance of the link. So if you wish to preferentially use limited-access and multilane highways, a penalty of 100 can be placed on the principal highway, through highway, and other road classifications, which will double the normal impedance for these road types. The route listing identifies modified lane types and the new penalty values.

\subsubsection{Nevada County Population Details}

In the lower right part of the Optional Highway Routing Parameters screen is a check box called Include Nevada County Population Details. This optional parameter provides population density and count data at the county level for routes passing through Nevada. With the activation of this feature, a population density table and population counts for Nevada counties appears following the standard population data information. Figure 3.11 shows and example of a portion of the population density table 
with the Nevada county data. A county code number represents data for each county. A key is provided at the end of the listing for each county name. To activate this option, click on this item to place a check mark in the box to the left of the option title. This feature is automatically included if the HRCQ+Nevada route type is selected.

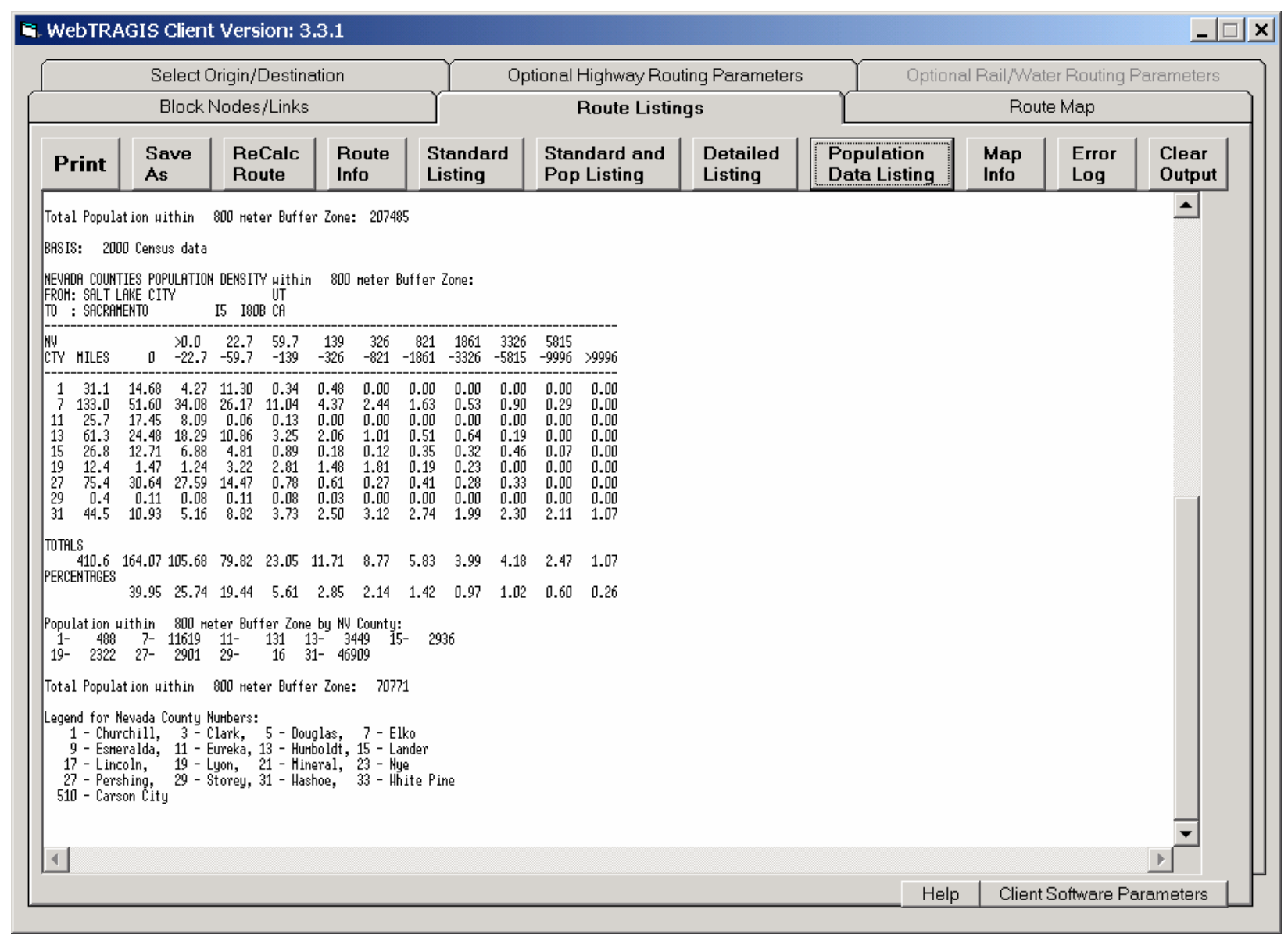

Fig. 3.11. Nevada county population density for a highway route.

\subsection{BLOCK NODES/LINKS SCREEN}

The Block Nodes/Links option is recommended only for experienced users. Selection of the Block Nodes/Links tab displays a screen that allows the user to temporarily modify portions of the highway network database. This feature can be useful for analysis of various scenarios such as road closures, construction zones, or damaged bridges. With this feature, the user can select nodes, links, or entire states to be excluded from consideration. Figure 3.11 shows the Block Nodes/Links window.

\subsubsection{Node Blocking}

Nodes can be blocked in the highway database thus making all the links that emanate from the node ineligible for routing. To block a node, first select the state by scrolling through the State list and then clicking on the desired postal abbreviation. The Node Name list will be populated with all the node names for that state. Next select the node to be blocked by scrolling to the name and clicking on it. In Fig. 3.12, the node at Exit 301 on I-80 west of Elko, Nevada is blocked. The node number for this 
location, "H 321100016," appears in the Blocked Node Numbers list. Node numbers are nine digits in length and are proceeded by a letter, in this case " $\mathrm{H}$," indicating the TRAGIS highway network. The right-most five digits are unique to the node within a state, and the leftmost two digits are the state Federal Information Processing Standard (FIPS) code for the state. For example, code 32 is Nevada. The third digit indicates the TRAGIS routing network ( 1 for highway, 2 for rail, and 3 for waterway) and the fourth digit indicates whether the feature is a node (1) or a link (2).

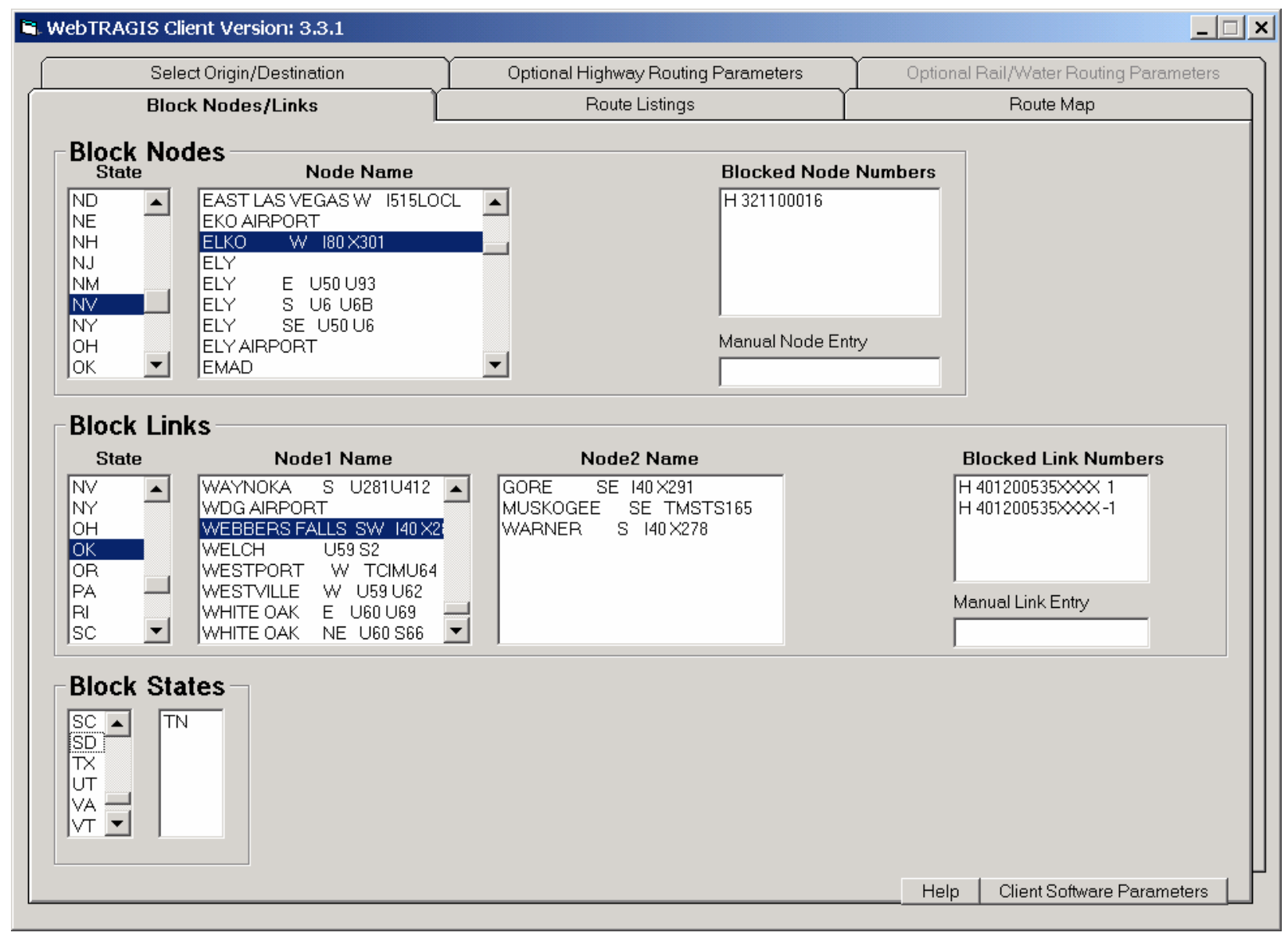

Fig. 3.12. Highway node/link blocking.

Additional nodes can be selected for blocking and they will also be listed in this box. When enough nodes are selected to fill this display box, a scroll bar will appear on the right hand edge to allow examination of the entire list. All nodes that are blocked are identified on subsequent route lists. A node number may be removed from the Blocked Node Numbers list by double-clicking on its entry.

The Manual Node Entry box appears below the Blocked Node Numbers box. This is provided if an unnamed node needs to be blocked. A node number can be determined by using the MapTips function on the Route Map screen (see Sect. 3.7.2). (Select MapTips, then select Hwynode in the box to the right of the text MapTips, and then select Nodenumb in the rightmost box. After moving the pointing device over a node, the node number will be displayed on the Route Map screen. See Fig. 3.3 for an example of identifying such nodes.) When entering an unnamed node number, only the numeric portion of the number needs to be entered. The number is added to the Blocked Node Numbers list after the enter key is pressed. 
Figure 3.13 shows the results of blocking the Elko node. The normal or green colored route in this figure shows a commercial route between Salt Lake City, Utah, and Sacramento, California. After the Elko node is blocked, shown with the triangle symbol, the model calculates the blocked or red colored route. The blocked route diverts from I-80 at the Nevada state line, heads south the Ely, Nevada, and then follows U.S. 50 across the central portion of the state. The blocked route returns to I-80 near Fernley.

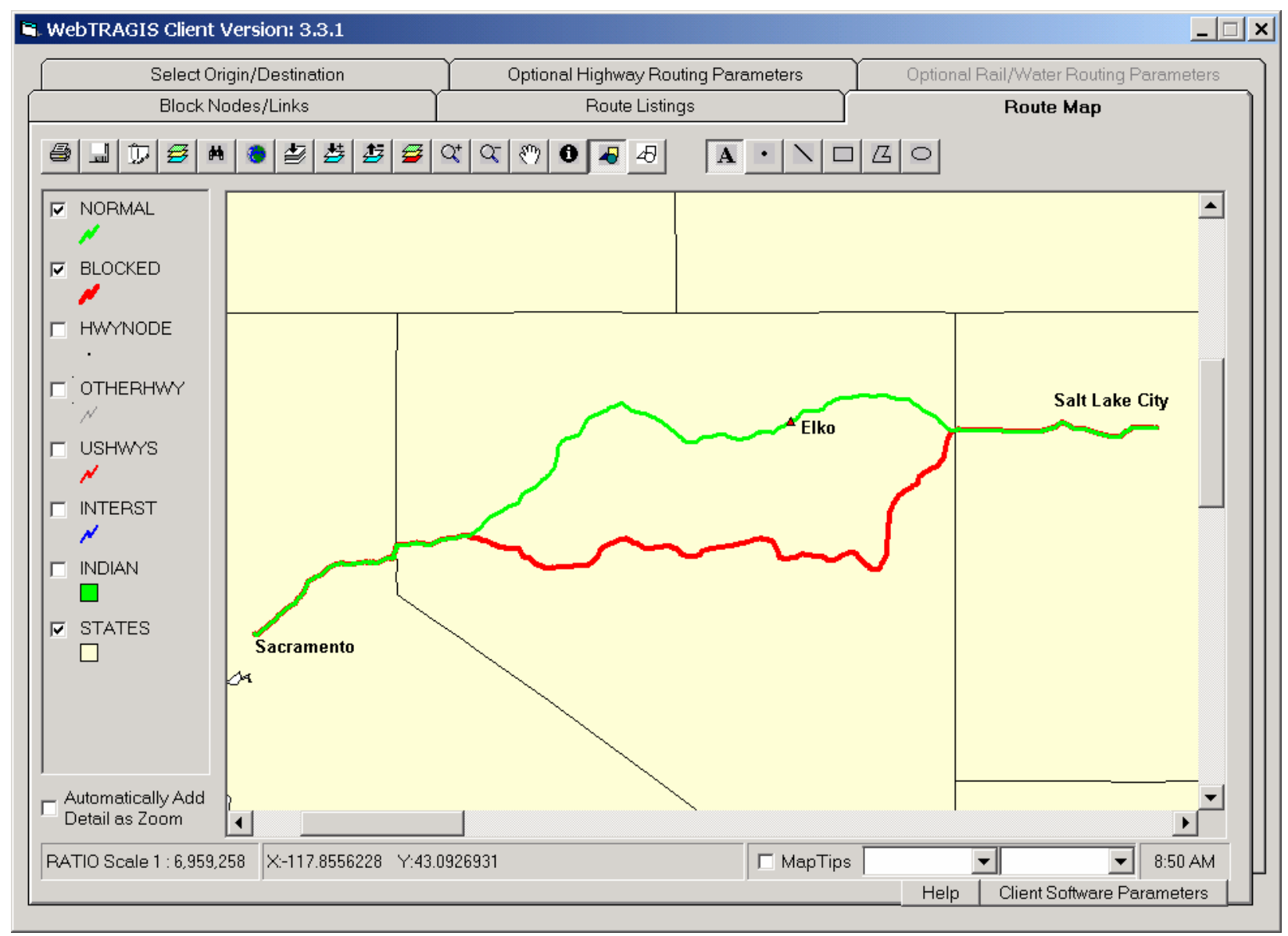

Fig. 3.13. Example of the result of blocking a highway node.

\subsubsection{Link Blocking}

Links in the highway database can be modified to temporarily remove them from consideration on a route. The textual method of blocking a link involves identifying the two end-node names. The first step is to select the state by scrolling through the State list and clicking on the postal abbreviation of the desired state. Next the Node1 Name list displays all the node names in this state. Select the desired node by clicking on the name. Following this, the Node2 Name list will be populated with the names of all the nodes connected to the highlighted location in the Node1 Name list. Select the appropriate location from the Node2 Name list by highlighting the name. The selected node name will be removed from the Node2 Name list and two sets of node numbers will appear in the Blocked Link Numbers list. Both entries will list the same link number, but the first entry ends with a ' 1 ' and the second ends with a ' -1 '. Links are represented in the model as one-way connections. The ' 1 ' link extends from the from-node to the to-node. Alternatively, the ' -1 ' link extends the other direction, from the to-node to the from-node. The easiest way to determine way to determine the direction of a link is to block the link and then run a route. The route 
listing provides a list of all blocked links and identifies the ' 1 ' link and the ' -1 ' link. If you want to block a link in only one direction, double click on the undesired entry, and it will be removed from the list.

On May 26, 2002, a barge collided with the I-40 bridge over the Arkansas River near Webber Falls in eastern Oklahoma. This bridge was out of service for 65 days while it was rebuilt. This provides a good example for a reason to be able to block a link. The example, shown in Fig. 3.12, demonstrates the removal of this segment of I-40. First the Oklahoma abbreviation is highlighted and the list of node names within that state is displayed in the Node1 Name list. Webber Falls SW I40 X286 is selected, which results in the display of the three nodes in the Node2 Name list. Gore SE I40 X291 is selected and the two link entries appear in the Blocked Link Numbers list. (During normal operation of WebTRAGIS, the entry for the Gore node will be removed from the Node2 Name list after selection to signal the user that the appropriate link has been selected. If numerous links have been blocked, it may not be clear to the user that the link has been marked for blocking.)

Figure 3.14 graphically shows the result of blocking the I-40 link over the Arkansas River. The normal or green colored line is a commercial route between Memphis, Tennessee, and Amarillo, Texas follows I-40 between these two cities. After blocking the I-40 link between Webber Falls and Gore, Oklahoma, the blocked or red colored route heads north on I-540 in western Arkansas and then follows U.S. 412 from northwestern Arkansas to the Tulsa area. The blocked route then follows I-44 to Oklahoma City, where this route rejoins I-40. (In reality, the actual detour utilized the U.S. 64 bridge over the Arkansas River near Gore. As mentioned in Sect. 3.1, the TRAGIS highway network does not include highways that closely parallel non-toll interstate highways and this bridge is not in the database.)

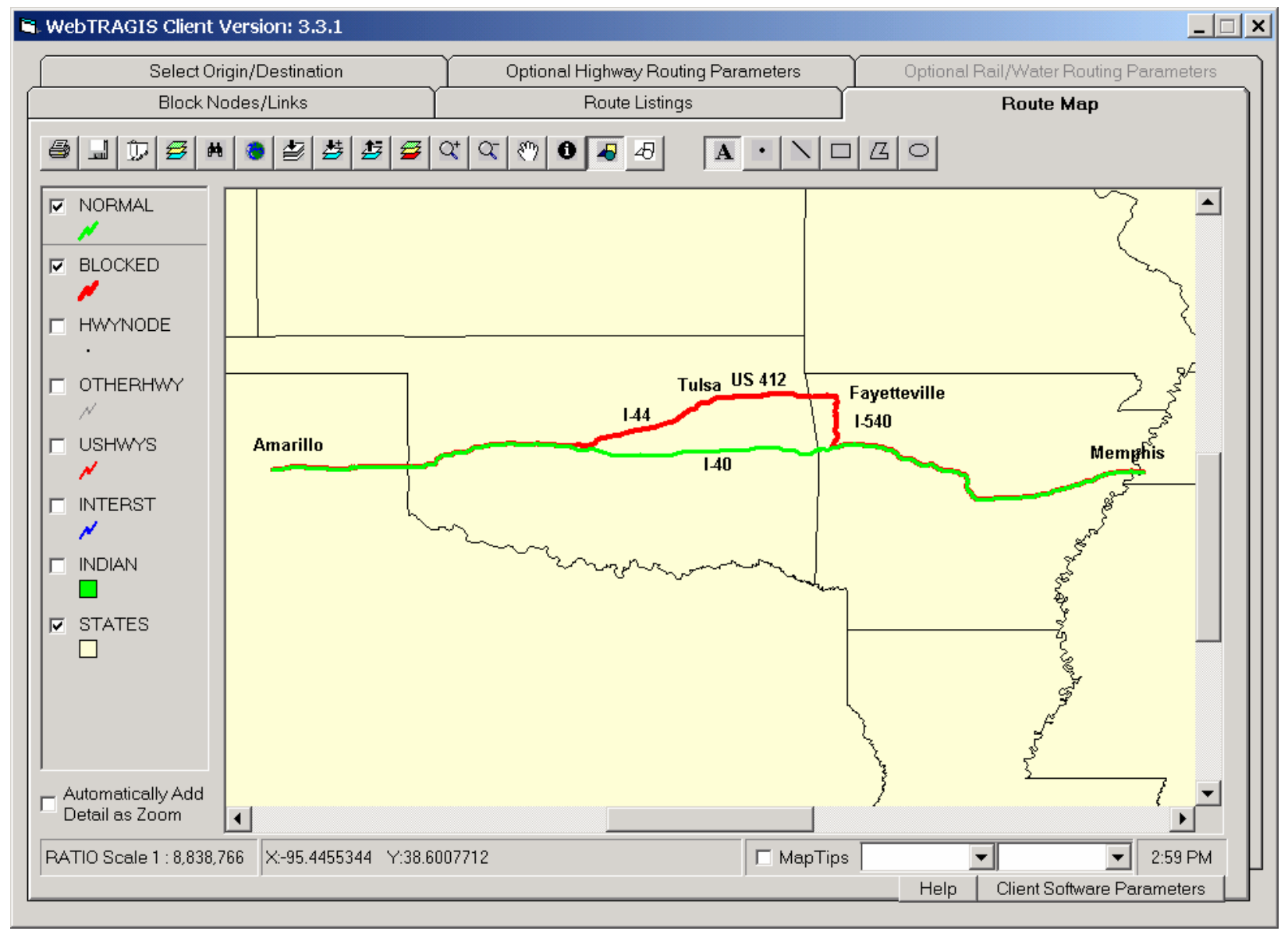

Fig. 3.14. Example of the results of blocking a highway link. 
The Manual Link Entry box appears below the Blocked Link Numbers box. This is provided to allow entry of a known link number. A link number can be determined by using the MapTips function on the Route Map screen (see Sect. 3.7.2). (Select MapTips, then select the appropriate link coverage, either Interst, Ushwys, or Otherhwy in the box to the right of the text MapTips, and then select Link in the rightmost box. After moving the pointing device over a link, the link number will be displayed on the Route Map screen.) When entering a link number, only the numeric portion of the number needs to be entered. The link number is added to the Blocked Link Numbers list after the enter key is pressed.

When links are blocked, the route listing identifies all links that are removed from consideration. The listing of blocked links also provides an easy method of determining the direction of a link because each link deletion results in two records - one for each direction. A link number may be removed from the Blocked Link Numbers list by double-clicking on the entry.

\subsubsection{State Blocking}

An entire state can be temporarily removed from the highway network database by selecting the state (or states) from the Blocked States list in the lower left corner of the Block Nodes/Links screen. The blocked states will appear in the right hand list. A state may be restored from the removal list by doubleclicking on the state abbreviation.

Figure 3.12 shows the lower portion of the alphabetical list of state abbreviations with Tennessee identified as blocked. Note that after Tennessee has been selected as a blocked state, that state's abbreviation is removed from the list in the left hand list box and placed in the blocked list to the right. When states are blocked a message is added to the route list identifying which states are removed from consideration during route calculation.

Figure 3.15 demonstrates the effect of blocking the State of Tennessee. The normal or green colored line is a commercial route between Birmingham, Alabama, and Indianapolis, Indiana, which follows I-65 between these two cities. After blocking Tennessee, the model cannot use any roads within the state. The resultant route between Birmingham and Indianapolis is shown as the blocked or red colored line in Fig. 3.15. This route heads northwest from Birmingham and across Mississippi taking the first highway bridge south of the Memphis area near Helena, Arkansas. The route then heads north on I-40 and I-55 through Arkansas and Missouri. In southern Illinois, the route continues on I-57 until reaching I-70 and then continues east to Indianapolis. This example shows the effect that blocking a state has upon the selection of a highway route.

\subsection{ROUTE MAP SCREEN}

Figure 3.16 displays the Route Map screen for the highway portion of WebTRAGIS. This section describes the functionality specific to the highway portion of the model. Section 6 of this user's manual provides a detailed description of common features to the highway, rail, and waterway Route Map screens.

\subsubsection{Highway Coverages}

The Route Map screen consists of the primary window displaying a map of the continental U.S. and a legend area on the left side of the window. Six coverages are shown in the legend-Hwynode,

Otherhwy, Ushwys, Interst, Indian, and States. The Hwynode coverage consists of points representing each node in the highway network database. Roads in the highway network are separated into three different coverages, primarily to simplify the map display. The three different road coverages are related 
to the sign type of the road; interstate highways, U.S. highways, and other highways. Native American tribal lands are shown in the Indian coverage. Finally, the State coverage shows the state boundaries. As seen in Fig. 3.16, the lower three coverages (Interst, Indian, and States) are checked, which indicates that these items are displayed in the screen. By checking or removing the check mark, a coverage is shown or not shown in the display window.

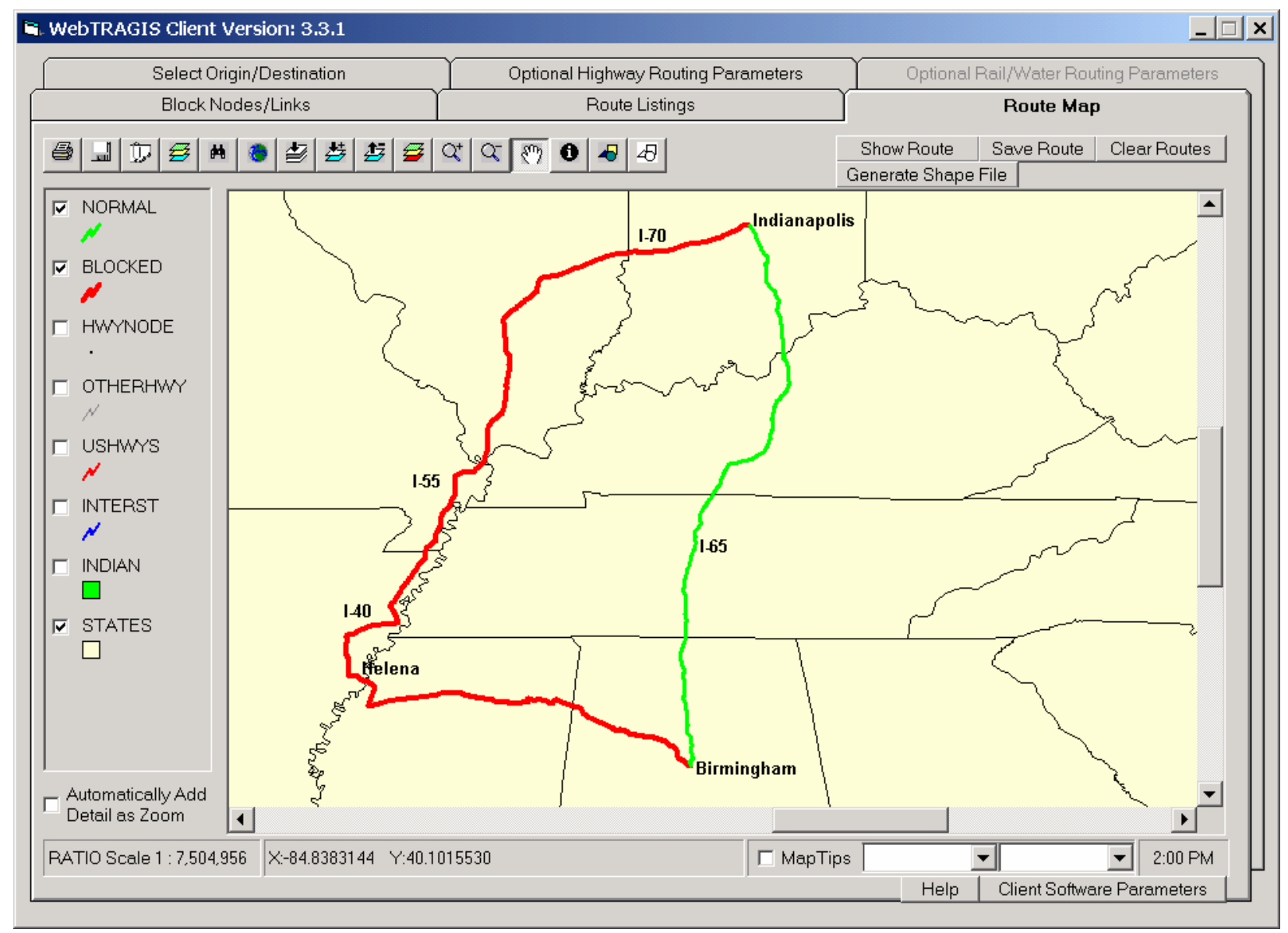

Fig. 3.15. Example of the result of blocking a state on a highway route.

\subsubsection{Using MapTips}

In the lower right hand center of the screen, MapTips has been checked, as shown in Fig. 3.16. (The MapTips item is checked for this example to assist the discussion of this feature. By default, MapTips is not checked when the Route Map screen is displayed.) Two pull down boxes appear to the right of the MapTips label. The left pull down box is used to select the coverage, in this case the Hwynode coverage. The right pull down box is used to select an individual attribute of coverage. When the Hwynode coverage is selected, the attribute field defaults to Name. MapTips is best used by zooming into an area and by checking all the coverages. Then move the pointing device over a node and the name of the node will be shown on the screen. 


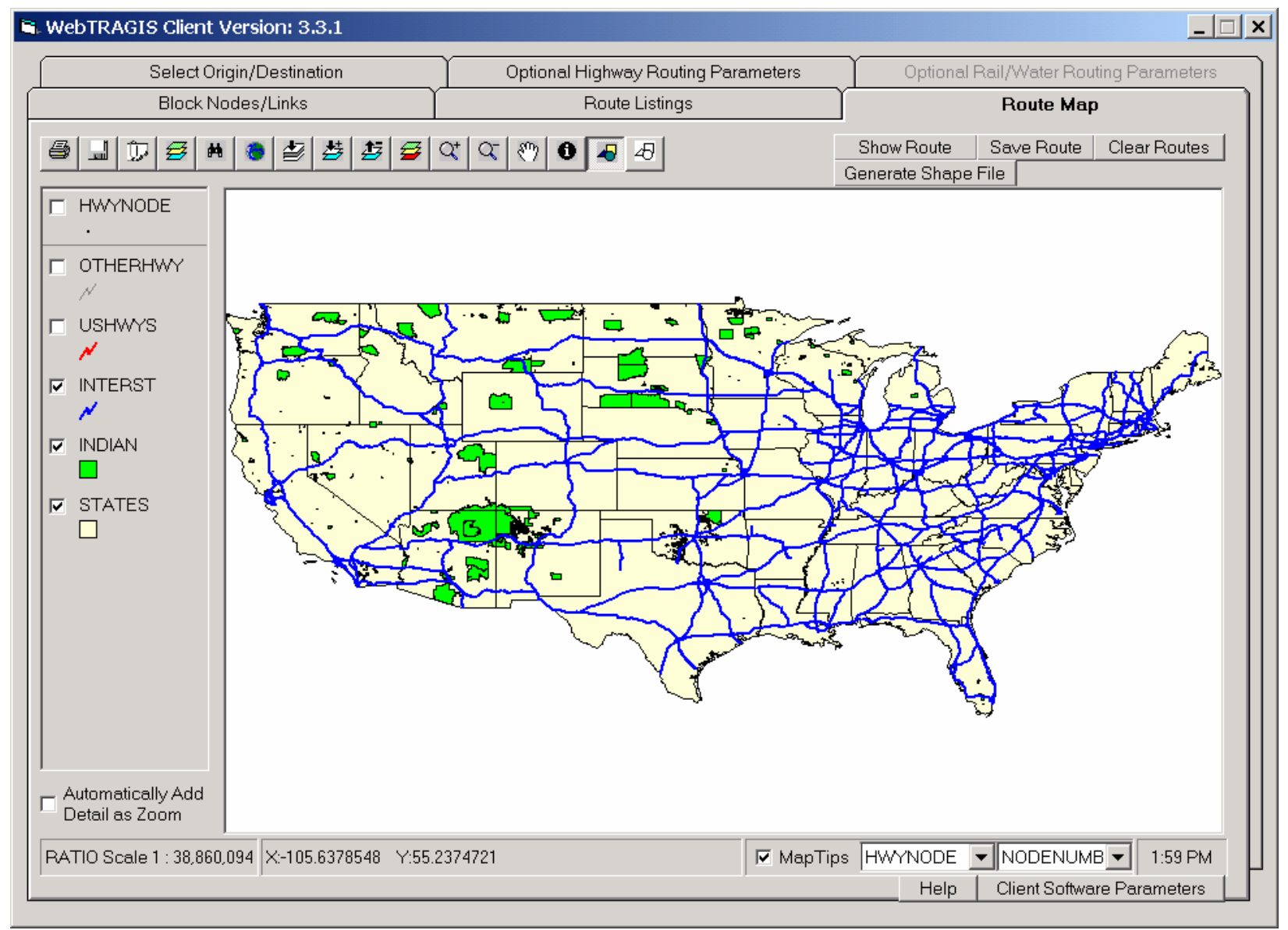

Fig. 3.16. Highway Route Map screen.

\subsubsection{Displaying Routes}

WebTRAGIS provides two methods of displaying a calculated route in the Route Map screen. The first is a simple and fast method that draws a stick figure representation of the route. The second takes more time, but displays the complete shape of the route. Figure 3.17 provides an example of the difference between the two methods. The route shown in this figure is I-40 passing through the Great Smoky Mountains along the North Carolina-Tennessee border. The green segments show the route representation using the stick figure method and the blue line is the shape file method. The red circles show the highway network nodes.

\subsubsection{Stick figure routes}

The simplest method draws straight-line segments between nodes. This technique is very fast and is sufficient for first draft national scale maps. The top row of buttons on the upper right hand portion of the Route Map screen is used to control the drawing of routes with this method. The Show Route button will display the most recently calculated route. (Even if you have just started WebTRAGIS, the most recently calculated route means the last route you have created with the model.) If you are creating a series of routes, the Show Route button can be pressed after the calculation of each to display multiple routes on the screen. Each successive route is displayed in a different color on the screen. (WebTRAGIS selects the color. The only way the user is able to change an undesirable color is to press Show Route another time.) The Save Route button saves all the routes displayed on the screen in one file in the ESRI shape file 
format. When this button is pressed, a save as window is opened to allow the user to select the folder where the file is stored. The Clear Routes button will clear all routes displayed on the screen that were added with the Show Route button.

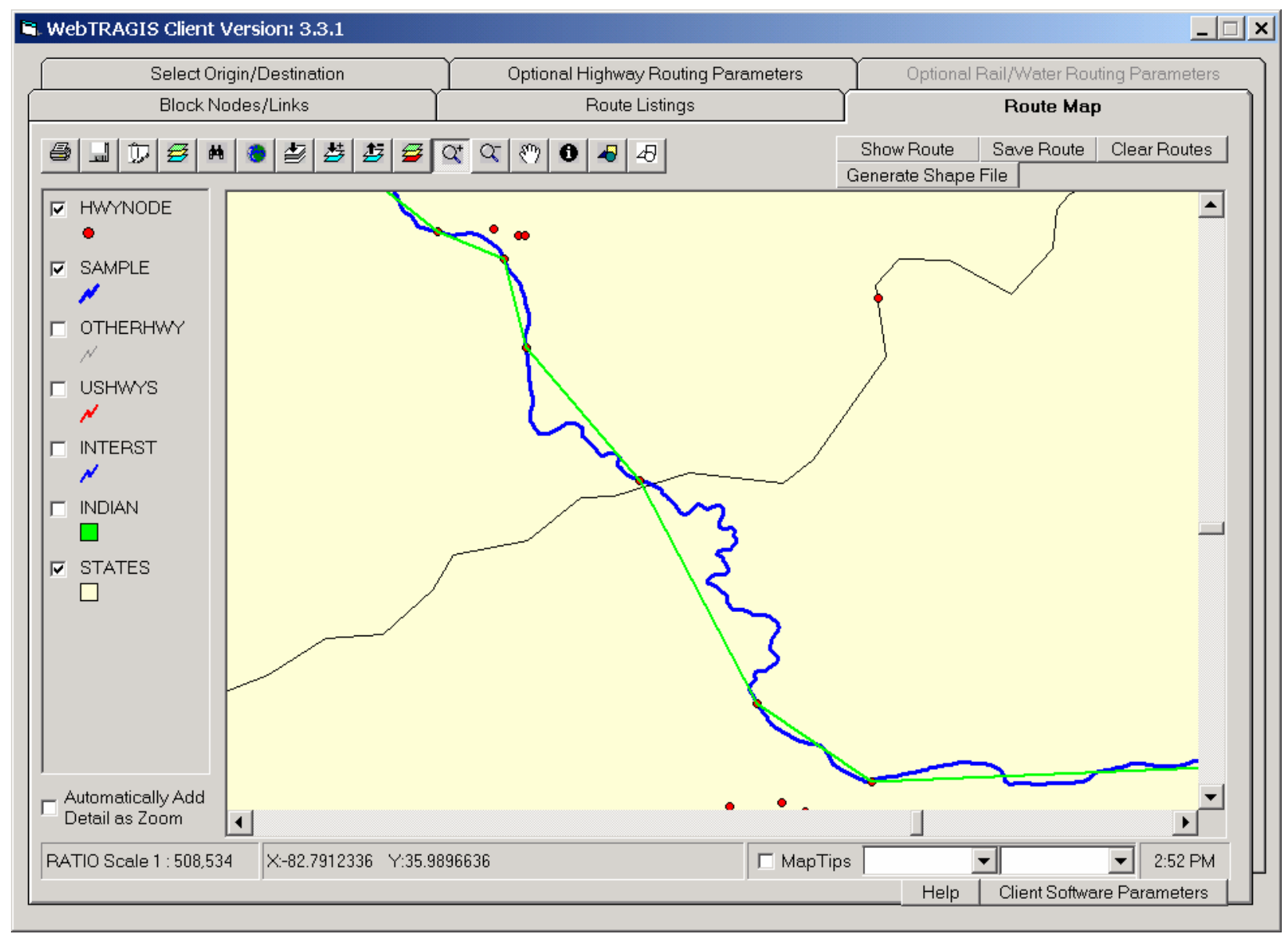

Fig. 3.17. Difference between the two highway route representations.

\subsubsection{Shape file routes}

The Generate Shape File button provides the capability to make a map of the route showing the full detail of the geographic shape available with the 1:100,000-scale highway network available in WebTRAGIS. This process is slightly more complicated compared to the method described in the previous section, but is the preferred method if large scale (showing small areas) maps are needed. When the Generate Shape File button is pressed a Save As window is opened, which provides the method for a unique file name to be entered. The file is saved in the ESRI shape file format, which can be used with ESRI GIS software, such as ArcView and ArcGIS. After entering the file name, press the Save button. After about a minute, the shape file will be created, displayed on the map, and an entry will be added to the legend. Click on the name of this new coverage to change the attributes, such as color and line width, of the route. To remove a generated shape file from the Route Map screen, select the coverage (click on the coverage legend so it appears to be slightly raised or highlighted) and then press the Remove Active Map Layer button, which is on the button bar (see Sect 6.1.9). 
Previously saved routes or other shape files can be added to the WebTRAGIS map display. Further information on this capability and other mapping features of WebTRAGIS is discussed in Sect. 6 of the user's manual. 
Page intentionally left blank 


\section{RAIL ROUTING}

The TRAGIS model calculates rail routes that simulate the routing practices of the railroad companies in the United States. The basic concept of determining rail routes is to calculate the shortest path based on travel distance biased by traffic density on rail segments. With highway routing, time and distance are primary factors. The highest speed roads are limited access and highway routes generally following such roads. With rail routing, traffic stays on the main lines, which have the highest traffic density. Another difference between highway and rail routing is ownership. Trucking companies can operate over any highway. Individual railroad companies can only move freight over lines they own or have permission to operate over.

Each segment of the rail-network database has a distance, in miles, and a variable signifying the volume of traffic density. The traffic-density information is divided into four classes as follows:

A-mainline-more than 20-million gross ton-miles per year,

B-mainline-between 5- and 20-million gross ton-miles per year,

A-branchline-between 1- and 5-million gross ton-miles per year, and

$\mathrm{B}$-branchline-less than 1-million gross ton-miles per year.

In addition to biasing the distance based on higher traffic density, the model also penalizes changing from one rail carrier to another. Finally, the model also reduces the impedance values on the originating rail carrier. These features replicate the practice of actual rail routing - which is that the originating carrier will attempt to keep the shipment on its system for as much of the total route as possible.

\subsection{OPERATIONAL CHARACTERICS OF THE U.S. RAILROAD SYSTEM}

Any mathematical model designed to predict rail transportation route must be capable of simulating the operation of the U.S. railroad system. This system is composed of a large number of independent companies that compete economically while simultaneously cooperating through interchange agreements to efficiently move freight across the country. Each company generally owns its own network of rail lines. In some instances, a company may have operating rights on a rail line owned by another company; this concept is referred to as "trackage rights." For more background information on railroad operations, read the book The Railroad: What It Is, What It Does (Armstrong 1998).

The overall network is, therefore, an aggregate of the interconnected track networks of hundreds of individual railroad systems. Private companies control virtually all railroads in the United States. Only a small amount of U.S. rail mileage is publicly owned, and most of this is dedicated to passenger operation-specifically commuter service in major urban areas. Private ownership of rail lines places constraints on the movement of commodities. No single railroad company is able to serve all possible origins and destinations that are of any significant distance apart within the country, and thus, at least two railroad companies must be used for most shipments.

The ability of railroad companies to freely exchange equipment between their networks is one feature of the U.S. rail system. Although any single company is limited to regional service, railroads cooperate by exchanging individual or blocks of freight cars moving to points beyond their individual service areas. These exchanges occur at designated transfer, or interchange, points where the lines of two or more companies meet. Sometimes interchange is handled by a third party, such as a terminal railroad. 
Interchanges are the bridges that connect the networks of the individual railroads thereby forming a national network.

Some locations are served by more than one railroad, and the shipper may choose which railroad will transport the shipment. This choice is based on a number of factors including cost, quality of service, and the railroad's willingness to provide service. While the shipper is, in principle, free to specify the railroads to be used as well as the interchange points, most shippers generally negotiate with a single railroad company, and that railroad will arrange to have the shipment interchanged with the other carriers involved in completing the shipment.

There are three aspects of realistically simulating common railroad routing practices. The first is the route that traffic will take between points on a single railroad system. The routing algorithm is designed to preferentially route a shipment on the rail lines having the highest traffic volume. High volume mainline routes are preferred because they are generally well maintained since the railroad depends on these lines for a major portion of its revenue (i.e., hence the name "mainline"). In addition, routing along the hightraffic lines usually replicates railroad operational practices.

The second aspect of rail routing is the selection of the sequence of railroads between the origin and destination. A delay is often involved in transferring a shipment from one railroad to another. While there are some run-through interchanges where the trains are not disassembled, the majority of interchanges require the incoming train to be disassembled and the cars to be sorted according to the receiving railroad. After the cars have been transferred to the receiving railroad's yard, they will be resorted according to destination and assembled into outgoing trains. In order to provide efficient service, the railroads try to reduce the time delays associated with interchanges by minimizing the number of interchanges in a route. This is accomplished in the TRAGIS model by imposing a numerical penalty for each interchange, which increases the apparent length of the route. Thus, when the model attempts to minimize the length of the route, it will also minimize the number of transfers to reduce the estimated time duration of a shipment.

The third aspect of routing on U.S. railroads is the desire of each individual company to maximize its portion of the shipment's associated revenue. This normally results in the originating railroad transporting the shipment as far as possible on its system before transferring the shipment to another railroad. This feature is represented in the TRAGIS model by the originating railroad preference. In evaluating the length of the route, the model treats 1 mile of travel on the originating railroad as being "less" than 1 mile on other railroads. (See discussion in Sect. 4.6.1 on the originating railroad factor.) This numerical adjustment increases the originating railroad's portion of the route.

\subsection{TRAGIS RAILROAD NETWORK}

The TRAGIS rail network is developed from a 1:100,000-scale rail network derived from the USGS digital line graphs. This network represents over 150,000 miles of rail lines in the continental U.S. Currently over 28,000 segments (links) and over 24,000 nodes exist in the database. All rail lines with the exception of industrial spurs are included in the network. Nodes have been included in the network for nuclear reactor, DOE sites, and military bases with rail access. The rail network has been extensively modified and is revised on a regular schedule to reflect line abandonment, company mergers, short line spin-offs, and new rail construction. Additionally, the agency that funded the development of this network requested that care be taken to ensure that turnouts at intersecting rail lines have the correct configuration for infrastructure analysis purposes. This rail network also includes the proposed alignments for the construction of a new rail line to the Yucca Mountain repository site. Because all of the options are included, the reader should examine Appendix $\mathrm{C}$ before calculating rail routes to Yucca Mountain. 
Many TRAGIS users may not be very familiar with the rail network in the U.S. There are currently over 500 different railroad companies in the country. Many of these railroads are very small and own a small amount of track. Such railroads are known as short lines. A few short lines have existed as small railroads since they were built, but most have been created over the past quarter century during the major consolidation that has occurred in the railroad industry. This consolidation has resulted in four major railroads in the U.S.-Burlington Northern Santa Fe Railway (BNSF), CSX Transportation (CSXT), Norfolk Southern Railway (NS), and Union Pacific Railroad (UP). These four major railroads operate over more than 60 percent of the track in the country. Each of these rail systems operates over more than 20,000 miles of track. Between the many small short line railroads and the four large systems are a few mid-sized railroad companies, which include the Canadian National Railways (CN), Canadian Pacific Railway (CPRS), and Kansas City Southern Railway (KCS). (CN operates on over 6,700 miles of track and CPRS operates over 4,300 miles track within the U.S. KCS operates on about 3,700 miles of track.) Nearly all the other railroads operate over less than 1,000 miles of track.

The TRAGIS rail network is organized into 97 sub-networks for routing purposes. This separation assists the routing algorithm in creating accurate routes. Appendix B provides a list of the different railroad companies in the rail network. In many cases, several short line railroads are owned by a parent company. These companies are grouped together into single subnetworks (e.g., R. J. Corman owns a number of small railroad companies; all are grouped into one subnetwork). With the large number of short line railroads, it would be impractical to provide separate networks for each rail company. The TRAGIS rail network has grouped these commonly owned railroads together. Three special sub-networks $(<\mathrm{C} 3>$, $<\mathrm{TR}>$, and $<\mathrm{OR}>$ ) are in the rail database. The $<\mathrm{C} 3>$ network (other Class 3 railroads) includes over 300 short line railroads not in other sub-networks. Many of these railroads have less than 50 miles of track. The $<\mathrm{TR}>$ network (other terminal railroads) includes about 70 small terminal railroads or other short line railroads. Again, these railroads involve a small amount of mileage. The final special network is $<$ OR $>$ (other rail lines). This sub-network represents ownership by non-common carriers and should not be used for routing purposes. Generally other rail systems will have trackage rights over these lines to provide rail service.

\subsection{RAILROAD NODE NAMING CONVENTIONS}

The TRAGIS rail network does not have a sophisticated node naming convention compared to the highway network. This network was developed from a network obtained from the Federal Railroad Administration. Many of the nodes have names identifying the city where the node is located. Other nodes are named for the railroad location, which may not be familiar to non-railroad personnel. These locations may be named after railroad employees, local roads, or railroad control points. In larger cities, several nodes may have the same name. This problem of non unique names will be dealt with as further modifications are made to the rail network.

Several node names may not appear in the node selection lists: nodes at county lines and at Indian reservation boundaries. Each node at a county boundary is called "County Line." Likewise, each node at the border of an Indian reservation is called "IRB" for Indian reservation boundary. Because of the large number of these non-unique names, these nodes have been removed from search list for selecting an origin or destination. If such a node needs to be used for an origin, destination, or blocking action, it can be selected by entering the unique node number in the manual entry boxes.

Nodes on state borders have unique names. Because state border nodes theoretically exist in two states, a special state name, BD for border, is established in TRAGIS to handle these nodes. An example of a state 
border node name is "IL-IN ST LINE CSXT 9." These node names, which consist of a maximum of 20 characters, follow a nomenclature of:

- first state abbreviation, followed by a dash (states appear in alphabetical order)

- second state abbreviation

- text "ST LINE"

- owner railroad abbreviation

- optional code (either numerical or alphabetic)

The optional code varies with the number of crossings by the railroad at a common state-to-state line. For the example provided above, CSXT has nine different crossings of the Illinois-Indiana border. With the numerical numbers, the numbering starts at the northern or western portion of the state boundary and works south or east. Alphabetic codes provide a descriptive code indicating that crossing is the east, west, north, south, or central of multiple crossings of a state line by one railroad company. Occasionally, a second alphabetic code is used to provide additional description. The second alphabetic code cannot be used if the railroad company abbreviation is four characters long because the length of the name would exceed the space available. If a railroad company only has one crossing of a state line, the optional code is not used.

\subsection{SELECT ORIGIN/DESTINATION SCREEN}

To set WebTRAGIS to calculate rail routes, the user needs to select the Rail option in the Mode section on the Select Origin/Destination screen. A delay of a few seconds will occur while the appropriate data files are loaded into the model. Figure 4.1 shows the initial display of WebTRAGIS in the rail mode.

\subsubsection{Selecting an Origin and Destination}

Two different methods can be used to select an origin and destination for a route. The first method is selecting a node name from the name list provided on the Select Origin/Destination screen. The other method involves selection of nodes by their number using the Route Map screen.

\subsubsection{Primary method-text}

In TRAGIS, the primary method of selecting an origin and destination for a rail route is to select the node name and railroad company within the respective scroll boxes. The order for selection of an origin and destination does not matter. First scroll through the State list and highlight the desired state by clicking on its abbreviation. Upon selection of a state, a list of node names within that state appears in the Node Name box. Scroll through the list and select the desired node name. After choosing a node name, the node number of that rail node is displayed in the respective Selected Node Number box. The final step is to examine the RR Company box to see if multiple railroads serve this location. If only one railroad is listed, the model automatically places this railroad abbreviation in the box to the right of the Selected Node Number. If multiple railroads are listed, one railroad needs to be chosen by clicking on the desired abbreviation. Once both are selected, the user may either press the Calculate Route button or select other parameters to modify the route.

Railroads are listed in alphabetic order in the RR Company list and represent either the railroad that owns the track at this location or the railroad(s) with trackage rights. If the user is uncertain as to which railroad should be selected, use the MapTips function on the Route Map screen to examine the Rrowner1 field of the Raillink coverage. Generally, the railroad that owns the track should be the rail system selected. 


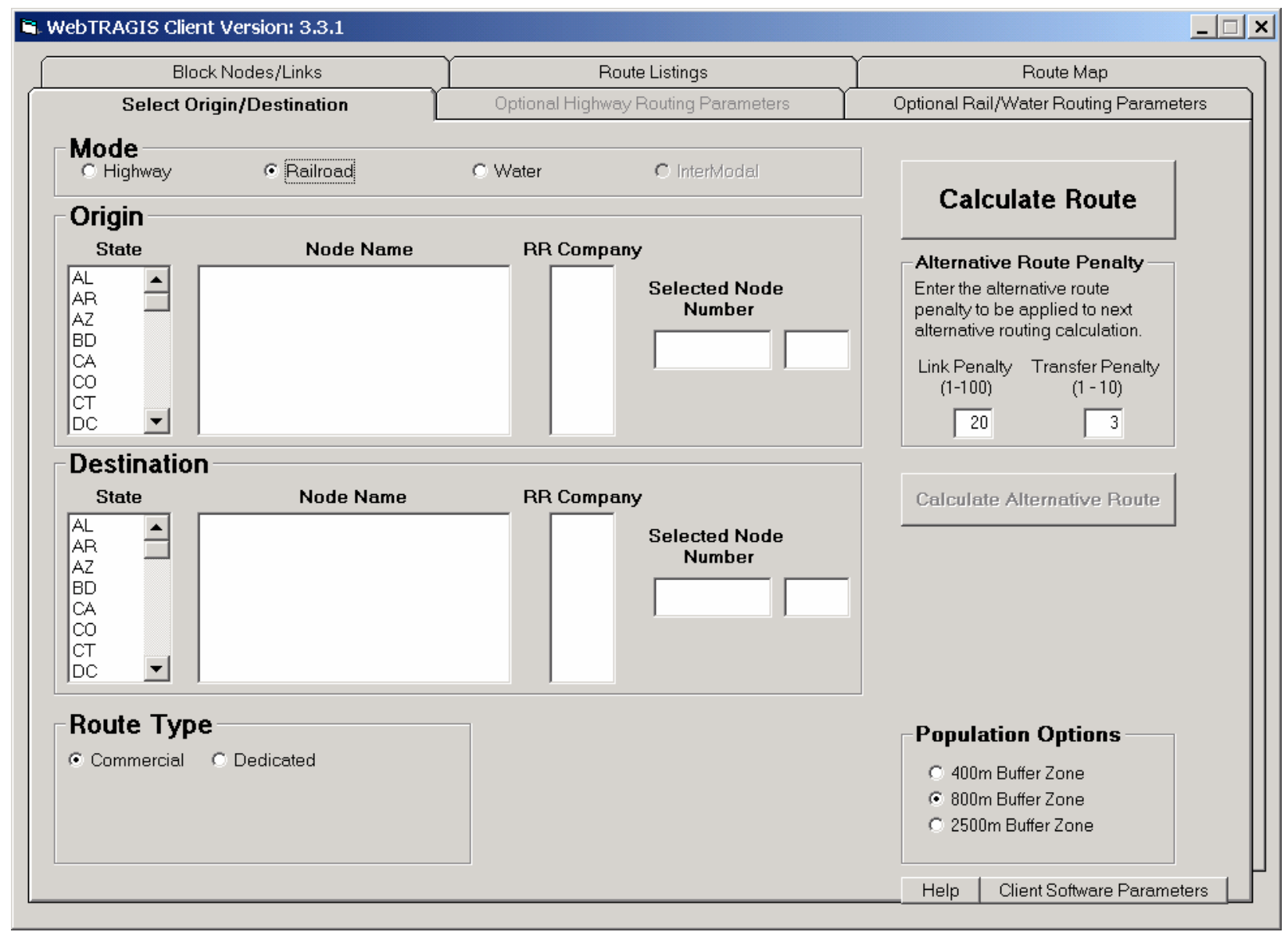

Fig. 4.1. Select Origin/Destination tab screen for rail routing.

Figure 4.2 shows an example of this method of selecting node names. In this example, the Origin box has the State of TN (Tennessee) highlighted, the Node Name list is scrolled to the end of the list (as is indicated by the position of the marker on the scroll bar), and the Y12 Plant node is highlighted. Two railroad companies are shown in the RR Company box as providing service and CSXT is selected. (In this particular example, if USG [U.S. Government] is selected, the model will not be able to successfully determine a route. The U.S. government owns the rail line to the Y12 Plant, but CSXT provides all rail service at the site. This type of situation occurs in the TRAGIS rail network on spur lines that are not owned by rail carriers.) The destination selected is the SRS Site C in South Carolina. This node is only served by USG (in this case the only railroad), so the model automatically selects this carrier. After the node number and railroad company are selected for both the origin and destination, the use may either press the Calculate Route button or select other user parameters to modify the route.

The previous example of an origin and destination, both served by USG, may appear confusing. The track at the Y-12 Plant is quite limited and there is not enough rail traffic to warrant onsite rail operations. For this reason, the large railroad company (CSXT) connecting to this spur line provides rail service over the USG owned track. At the Savannah River Site conditions are different and the government operates trains over the onsite tracks. Appendix D provides a list of various sites, the proper node to use, and the rail carrier providing service at the facilities. 


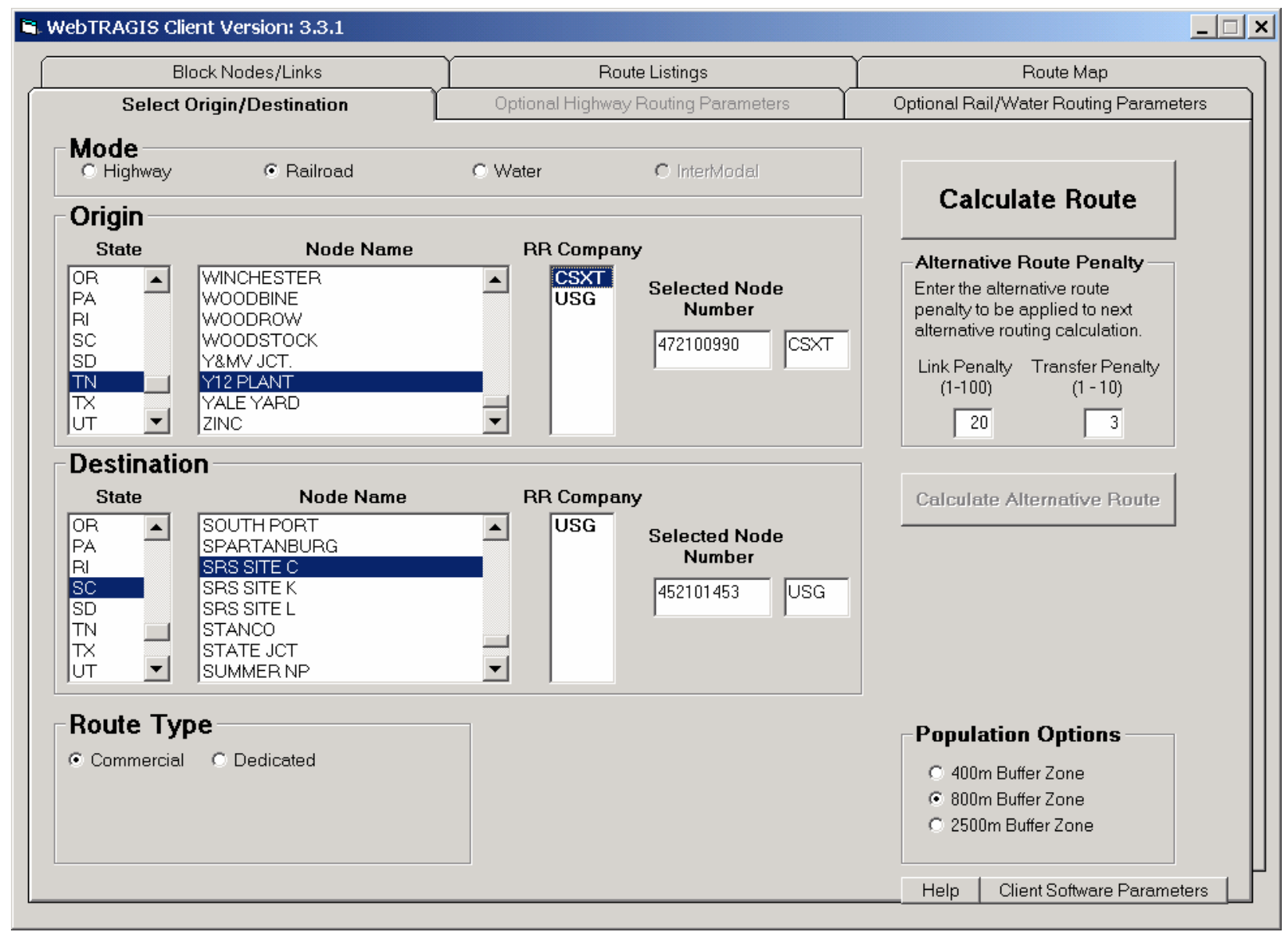

Fig. 4.2. Selection of a rail origin and destination.

\subsubsection{Alternative method-node number}

An alternative method to select an origin and destination is to enter a specific node number. This method may need to be used when a node does not have a name or when several nodes have the same name. Not all nodes are named in the TRAGIS rail network. Some named nodes do not have unique names. For example, links within the rail network have nodes at county boundaries. The common name of these nodes is "County Line." Also nodes exist where rail lines cross tribal lands ("IRB"). Finally, common names may exist within a city.

Figure 4.3 provides a zoomed in view of the rail network in Knoxville, Tennessee. The three nodes that are named Knoxville are identified by the triangle symbol, along with their name and respective node number. The node list method, described in Sect. 4.4.1.1, identifies each these three Knoxville nodes. If any of these three "Knoxville" are selected from the Node Name list, only node 472100958 would be selected, which is the northern node identified as Knoxville on Fig. 4.3. This problem will to be addressed over time by changing such node names to create unique names. At this time, if either of the other two nodes needs to be selected, the node number method of selection described here should be used.

To determine a node number, click on the Route Map tab to view the rail network. Use the zoom in tool to enlarge the area of interest. Next, click on the MapTips feature found on the lower right hand portion of the screen and select the RailNodes coverage and the NodeNumb field. (The down arrow buttons 
display a list of the available coverages and fields available for viewing.) Move the pointer over a node and the node number will be displayed on the screen. In Fig. 4.3, the pointer is positioned over the node in the lower portion of the screen and the node number 472101033 is displayed just below this node. After determining the appropriate node number, manually type it in the appropriate Selected Node Number box on the Select Origin/Destination screen.

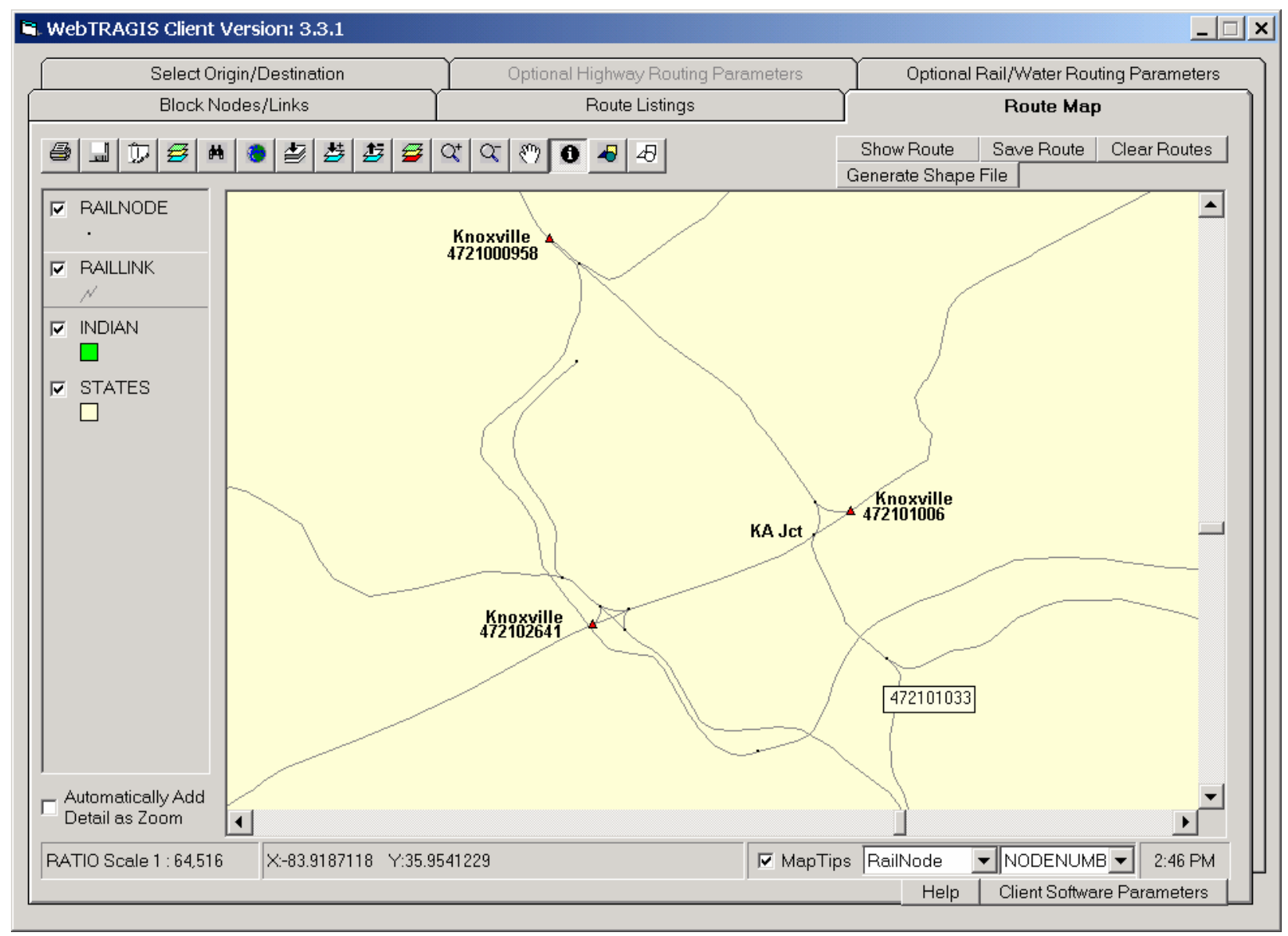

Fig. 4.3. Example of multiple non unique rail nodes names in Knoxville.

The railroad company also needs to be entered for this node, as described in this paragraph. Depending upon the complexity of the rail network in the area, the user should examine the link attributes of those links connecting to the selected node. Since several attribute field contain railroad ownership and trackage rights information, the best method to examine the link attributes is to use the identify button on the Route Map screen. (This button is described in Sect. 6.1.14.) The identify tool is activated by pressing the identify tool button (i.e., the lowercase "i" inside a black circle and selected in Fig. 4.3). Next click on the Raillinks coverage in the legend area on the left side of the screen so that it appears raised (also shown in Fig. 4.3). Now move the identify pointer to a link connecting to the selected node and click on the link. The link should flash several times and then an Identify Results window appears. This window provides a list of all the attributes for the identified link. Scroll through the list of attributes and examine the railroad owners and trackage rights attributes-Rrowner1, Rrowner2, Rrowner3, Tr1, Tr2, Tr3, Tr4, $\operatorname{Tr} 5$, Tr6, and Tr7. Most of these attributes may not contain values, but be sure to check the Rrowner1 and Tr1 fields. The Rrowner1 abbreviation is the best choice to use. Enter the appropriate railroad abbreviation, in upper case, in the box to the right of the Selected Node Number on the Select Origin/Destination screen. Care should be taken with short line railroads, because the rail network 
attributes display the individual company. As mentioned in Sect. 4.2, smaller railroads may be combined with other companies or placed in the catchall sub-network of other Class 3 railroads. Check Appendix B for the grouping of railroad companies.

Figure 4.4 shows the results of identify tool on the link between KA Junction node and node number 472101033 shown in Fig. 4.3. The owner of this link (signified by the Rrowner1 attribute) is NS and the Knoxville and Holston River Railroad (KXHR) has trackage rights (as indicated by the Tr1 attribute). The other ownership and trackage rights fields do not have data. By examining Table 2 in Appendix B, it can be determined that the KXHR is represented by the Georgia and Florida Railnet (GFRR) rail network. Table 1 in Appendix B shows that the GFRR and seven other railroads is owned by the Gulf and Ohio Railways.

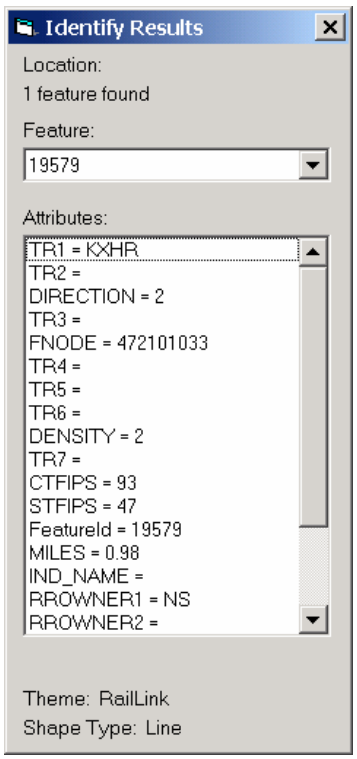

Fig. 4.4. Identify Results window for rail link attributes.

\subsubsection{Route Type}

The Route Type selection box is located in the lower left portion of the Select Origin/Destination screen, as shown in Fig. 4.2. This option sets the type of rail route that the model will calculate. Two choices are available - the default commercial (manifest freight) or dedicated train. To switch between the two options, click on the appropriate item.

\subsubsection{Commercial train route type}

The commercial train route type is the primary route type to determine route routes. The TRAGIS model calculates rail routes by minimizing the total impedance between the origin and the destination. The impedance is defined as a function of distance, mainline classification, and number of railroads involved in making the shipment (represented by the transfer penalty). Mainline classification for each link is based on the traffic density, discussed at the beginning of this chapter. The model identifies the set of links between the origin and destination that minimizes the impedance:

$$
L=\operatorname{Min}\left\{\sum_{i}\left(\sigma_{i} f_{i} d_{i}\right)+\sum_{n}\left(T_{n}\right)\right\},
$$

where 


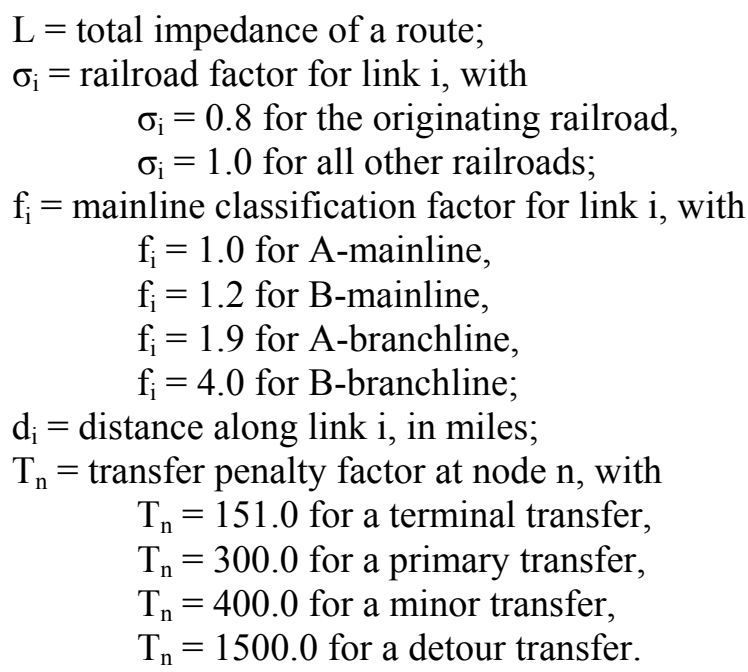

With the commercial route type, the routing algorithm shown in Eq. (3) preferentially routes a shipment along the A- and B-mainlines, while minimizing interchanges between railroad companies. In general, shipments will utilize A- and B-branchlines only as a connection between the mainline network and the origin or destination. Frequently, several railroads will provide service at the same location. Selection of an originating railroad has an impact on the estimated route because the originating railroad will preferentially attempt to move the shipment on its own system before interchanging with another railroad in order to maximize its portion of the revenue. Because of the originating railroad factor, commercial rail routes are not necessarily symmetric. A different route may be determined if the origin and destination are reversed.

The new 1:100,000-scale rail network identifies four different types of interchange locations between railroads: terminal, primary, minor and detour. In the previous versions of TRAGIS and the legacy INTERLINE model, interchange locations were only treated as standard transfer locations with a penalty of 300 units or as terminal transfer with a penalty of 151 units. Terminal transfer locations remain the same with the current rail network, but the previous standard interchange locations are now either designated as primary or minor transfers. Some of the previous standard transfers remain the same but are now called primary transfers. Interchanges that are not used for high volume interchange between major rail systems are now designated minor transfers and have a higher penalty of 400 units. Detour transfers did not exist in the previous rail network. Detour interchanges have physical connections between two different rail systems, but are not used to interchange freight traffic. These sites are used to move trains to the other rail system during rare traffic delays as a result of line blockages due to derailments or maintenance activities. The penalty for use of a detour transfer is quite high at 1500 units. Under normal conditions, a detour interchange will not be used on a route.

\subsubsection{Dedicated train route type}

The dedicated train route type provides a variation on commercial train route type. With a dedicated train route, the originating railroad factor is not considered and the transfer penalty factor is reduced by a factor of ten. The impedance equation for calculating a dedicated train route is:

$$
L=\operatorname{Min}\left\{\sum_{i}\left(f_{i} d_{i}\right)+\sum_{n}\left(\frac{T_{n}}{10}\right)\right\},
$$

where

$$
\mathrm{L}=\text { total impedance of a route; }
$$




$$
\begin{aligned}
& \mathrm{f}_{\mathrm{i}}=\text { mainline classification factor for link } \mathrm{i} \text {, with } \\
& \mathrm{f}_{\mathrm{i}}=1.0 \text { for A-mainline, } \\
& \mathrm{f}_{\mathrm{i}}=1.2 \text { for B-mainline, } \\
& \mathrm{f}_{\mathrm{i}}=1.9 \text { for A-branchline, } \\
& \mathrm{f}_{\mathrm{i}}=4.0 \text { for B-branchline; } \\
& \mathrm{d}_{\mathrm{i}}=\text { distance along link } \mathrm{i} \text {, in miles; } \\
& \mathrm{T}_{\mathrm{n}}=\text { transfer penalty factor at node } \mathrm{n}, \text { with } \\
& \mathrm{T}_{\mathrm{n}}=151.0 \text { for a terminal transfer, } \\
& \mathrm{T}_{\mathrm{n}}=300.0 \text { for a primary transfer, } \\
& \mathrm{T}_{\mathrm{n}}=400.0 \text { for a minor transfer, } \\
& \mathrm{T}_{\mathrm{n}}=1500.0 \text { for a detour transfer. }
\end{aligned}
$$

The effect of the dedicated train route type is that rail routes become more symmetric. Also more direct routes are chosen due to the lower impact of transfer penalties. The dedicated train route type should only be used if a dedicate train is expected to be used for shipments. In some cases, this route type will generate the same route as the commercial train route type.

\subsubsection{Population Options}

In addition to route selection, one of the most important applications of TRAGIS is to provide population density and count data along routes. The population data in TRAGIS are derived from the LandScan USA 15-arc second (approximately 360 by 460 meter) grid cell population database (Bhaduri 2002). This database represents nighttime population distribution and is developed from a combination of data sources including 2000 U.S. Census Bureau block group population, roads from the Census TIGER data, slope from the NIMA Digital Terrain Elevation Data, and land cover from the USGS National Land Cover Database. Population density and count information are based on geographic buffers constructed around the transportation route. Data on three different buffer distances are available in TRAGIS - 400, 800 and 2500 meters either side of the route. By default, the model selects the 800 meter-buffer width. To examine the population within one of the other corridor distances, select the appropriate distance within the Population Options box in the lower right hand portion of the Select Origin/Destination screen. Please note that the selection of a different buffer distance will not be executed until the Calculate Route button is pressed. The population along a route is displayed using the Population Data Listing button on the Route Listings screen. (See Sect. 4.5.8 for a description of the output data.)

\subsubsection{Calculate Route}

After an origin, destination, route type, and other options are selected, click the Calculate Route button on the right side of the screen. If a required item has not been selected, an error message window will appear with a reminder that all origin and destination data need to be provided before a route can be calculated. WebTRAGIS will take a few seconds to calculate the rail route and then display the Route Listings window showing the standard listing for the route.

\subsubsection{Calculate Alternative Route}

After a route has been calculated, the Calculate Alternative Route button becomes active, allowing the user to generate alternative routes. This feature is useful to determine if additional routes exist that are similar, but different than the optimal route selected by WebTRAGIS. In preparation for running an alternative route, all links comprising the current route are penalized by the Alternative Route Penalty factors. There are two separate alternative route penalty factors for rail routing-link and transfer penalties. The default value for the Link Penalty is 20 and 3 for the Transfer Penalty. The Link 
Penalty adds an additional 20 percent to the existing impedance of each previously used link. The Transfer Penalty multiplies used interchange penalties by a factor of 3 . Due to the increased impedance values, alternative routes will tend to use a different set of links and interchange locations. When running multiple alternative routes, the penalization of multiple previously used links and transfers are multiplicative. Entering a new value in the Link Penalty and Transfer Penalty areas of the Alternative Route Penalty box, located between the Calculate Route and Calculate Alternative Route buttons, can change these factors. Care should be taken in adjusting these factors.

To calculate an alternative route, go back to the Select Origin/Destination tab and then click the Calculate Alternative Route button. The number of viable alternative routes that TRAGIS can calculate is dependent upon the density of the rail network between the origin and destination. A sparse network may not provide any other path. In that case, the model will display the same route. (No message is provided indicating that an identical route was determined.) In other cases, where there is more connectivity in the network, the model may provide several alternative routes. However, repeated attempts to calculate numerous alternative routes may begin to lead to nonsensical results. Another less automated (but more frequently used) method to determine alternative routes is to block certain links, nodes, states, or railroad systems from consideration.

\subsection{ROUTE LISTINGS SCREEN}

The Route Listings screen provides access to the text-based output from WebTRAGIS. When the model generates a route, the program displays the Route Listings screen with the standard listing of the calculated route, as shown in Fig. 4.5. A number of buttons are located along the upper edge of this screen. The function of each of these buttons is provided below.

\subsubsection{Print}

The Print button sends the information listed in the Route Listings screen to the printer connected to the PC running the client version of WebTRAGIS. The default windows printer and settings are used to print data displayed on the screen.

\subsubsection{Save As}

The Save As button provides a method of writing the information listed in the Route Listings screen to a standard text file. The function of this button is the same for whatever previous button is used to display data on this screen, be it the Detailed Listing, Population Data Listing, Route Info, or Standard Listing button. When this button is pressed a Save As window is opened, which allows the user to select the appropriate drive, directory, and file name where the text information from the screen will be saved. By default, this function saves all files with the ".txt" extension.

\subsubsection{ReCalc Route}

The ReCalc Route button opens a window to select a previously saved Route Info file. After this file is selected, WebTRAGIS will recalculate the route based on the parameters in the Route Info file. The normal user will rarely, if ever, need to use this feature. This feature is primarily used to assist users who are having problems with the model and allows TRAGIS development staff to be able to reproduce user problems. 


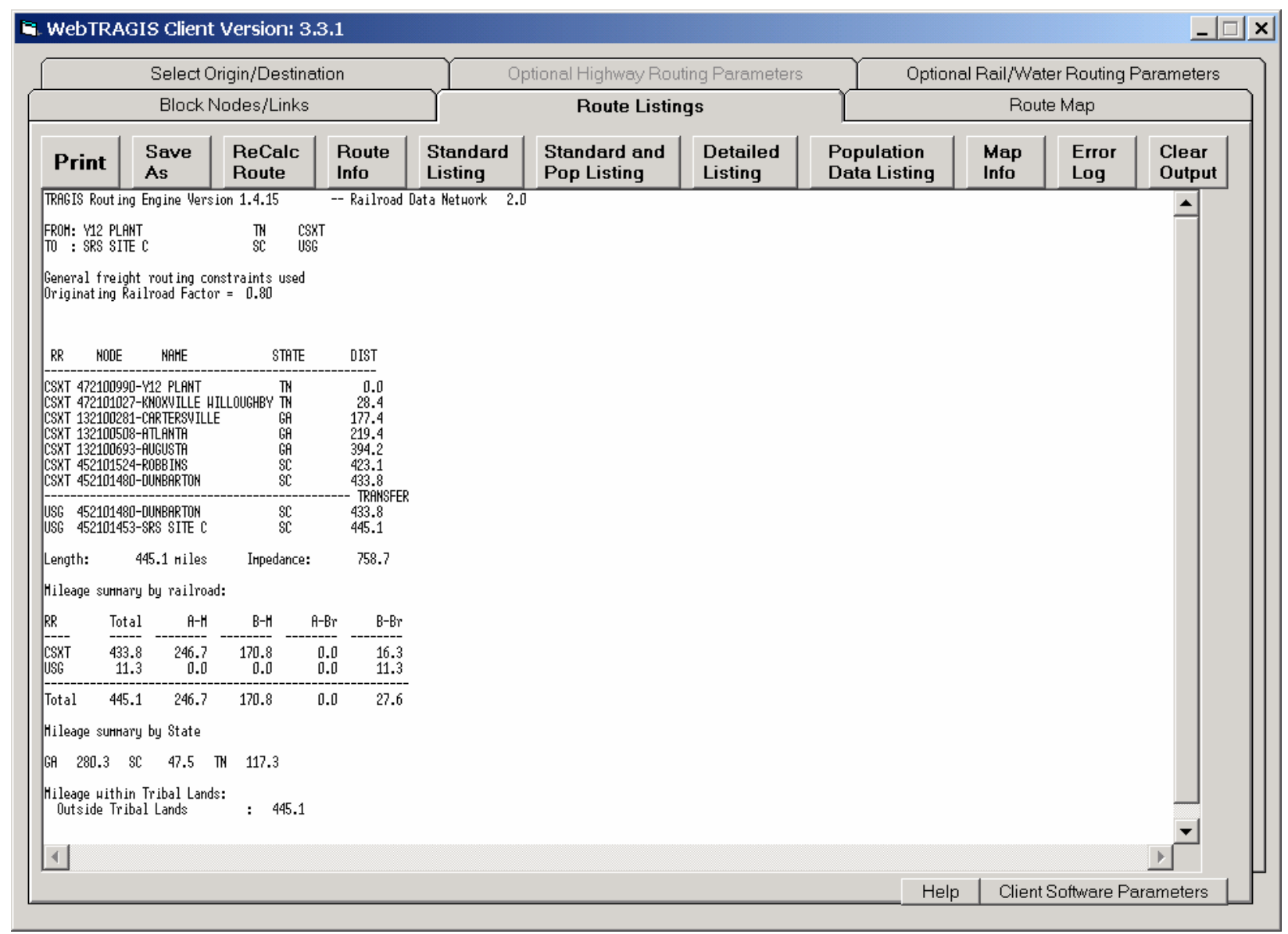

Fig. 4.5. Standard rail route listing.

\subsubsection{Route Info}

The Route Info button displays the most recent set of parameters used to create a route (such as the origin, destination, option settings, blocked nodes and links, etc.). This set of parameters is sent over the Internet to the TRAGIS server to calculate the route. If the user experiences any problems with generating a particular route, this button should be used to examine the parameter settings. If further assistance is required, the Route Info data should be saved in a file, using the Save As button, and then the saved file can be e-mailed to the TRAGIS development staff for debugging.

\subsubsection{Standard Listing}

The Standard Listing button displays the route description of the most recent route calculated. (WebTRAGIS displays this listing following the calculation of each route.) Figure 4.5 provides an example of the standard listing of a route between the Y-12 Plant in Tennessee and the Savannah River Site in South Carolina.

This route listing identifies the origin and destination, any transfer locations, and larger cities along the route. The main part of the route listing identifies the railroad company used for each portion of the route, the node number and station name of each record in the display, and a cumulative mileage figure for each location listed. Following the route list is a summary of the total distance and the total impedance 
(mathematical summation) of the route. This is followed by a breakdown of mileage by railroad and mainline classification and a summary of mileage within each state that the route passes through. The final portion of the listing is a mileage summary of any Native American Tribal lands encountered by the route.

In Fig. 4.5, CSXT moves the shipment from the Y-12 Plant through Knoxville, Atlanta, and Augusta to Dunbarton, South Carolina, where the shipment is transferred to the USG for the final 11.3 miles to Site C in the Savannah River Site. This route has a total length of 445.1 miles.

\subsubsection{Standard and Pop Listing}

The Standard and Pop Listing button displays a combination of the information shown with both the Standard Listing (see Sect. 4.5.5) and the Population Data Listing (see Sect. 4.5.8) buttons.

\subsubsection{Detailed Listing}

The Detailed Listing button provides additional information on the rail route. Information is similar to the standard rail listing, but with the detailed rail listing every node and link that makes up the route is identified on the display along with information on link characteristics. All node numbers are listed including those nodes without names. Link information includes the link number; the distance of the link; the line classification code; a traffic volume code; if the operating railroad is using trackage rights over this line, the abbreviation of the railroad owning the tracks; and the impedance for the link. As with the standard listing, summary information by railroad, state, and tribal lands follows the route listing.

Figure 4.6 provides and example of a detailed rail route listing between the East Tennessee Technology Park (the former Oak Ridge K-25 Gaseous Diffusion Plant) and Oliver Springs, Tennessee. A short line called the Southern Freight Railroad, which is grouped into the other Class III railroad system (see Appendix B, Table 2), designated as " $<\mathrm{C} 3>$," serves the East Tennessee Technology Park. The shipment moves over one link to Blair, Tennessee. The attributes shown for this link include a distance (Dist) of 5.1 miles, a mainline classification (LC) of 4 (B-branchline), traffic density (Den) of 1 (less than 1 million gross ton-miles per mile annually), the line is owned (Owner) by the operating railroad, and the Impedance is 16.45 units. At Blair, the shipment is transferred to the NS and the transfer penalty is 300 units. The remainder of the route has a mainline classification of 1 (A-Mainline) and a density level of 5, which represents a traffic density of 20 to 30 million gross ton-miles per mile annually. The NS moves the shipment between Blair and Harriman, where it is transferred to the CSXT. The NS to CSXT transfer penalty at Harriman is 400 units. CSXT moves the shipment from Harriman to Oliver Springs. Please note the NS designations on the CSXT portion of the listing between Harriman and Oliver Springs. The NS in the Owner field between these locations indicates that CSXT operates over NS owned tracks.

\subsubsection{Population Data Listing}

The population-density information is viewed by clicking the Population Data button. A listing of the population density information for the route is displayed. Figure 4.7 shows the population data listing for a route between the Y-12 Plant and SRS Site C route shown in Fig. 4.5. The basic table includes 11 population-density categories ranging from 0 to over 9996 people per square mile. The entries in the table show the distance that the route travels in each category. At the end of the table, summary information is provided for the route. This information combines the data from the 12 categories into rural, suburban, and urban groups and also provides a weighted population-density value for each of these groups. This information is used as route specific input for risk analysis using the RADTRAN (Neuhauser 2000) or RISKIND (Yuan 1995) computer codes. Finally, population count data are provided for the route by state 
and for the entire length of the route. For the example shown in Fig. 4.7, 303,443 people reside within 800 meters either side of this route.

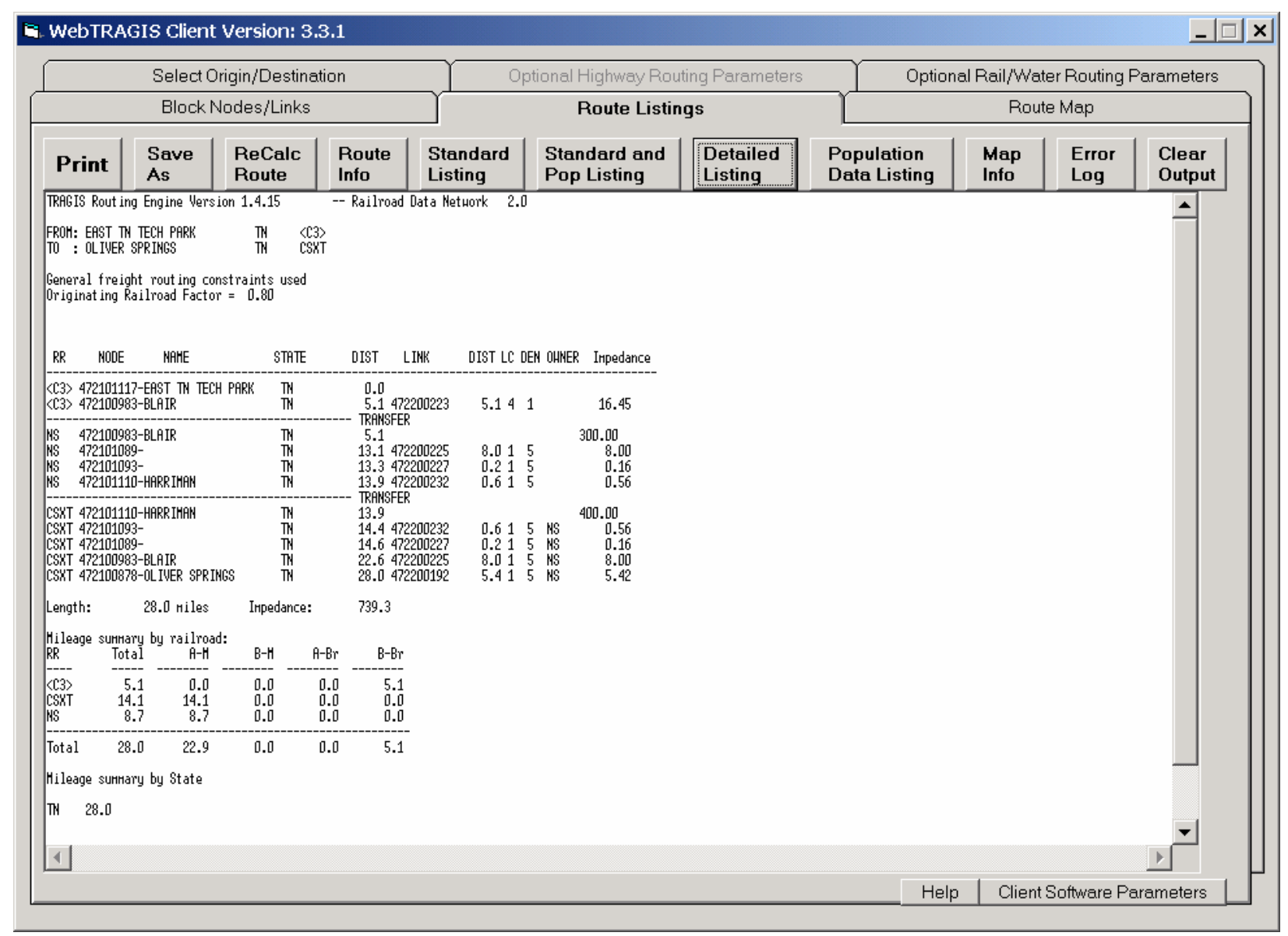

Fig. 4.6. Detailed rail route listing.

Input data for the RADTRAN model requests population density and distance data for rural, suburban, and urban areas. The basis for these three categories is:

- Rural

Categories below 139 people per square mile,

- Suburban

Categories between 139 and 3326 people per square mile,

- Urban

Categories above 3326 people per square mile.

The model reports the route specific weighted population density and distance data (in both English and metric units) for the three population categories.

With the improved calculation methods used to determine the population numbers, the user should note that these data are only estimates. Also, these numbers represent nighttime population figures and that daytime population distributions are significantly different. It is best to use these population figures for global comparisons of different routes rather than an absolute value.

\subsubsection{Map Info}

The Map Info button displays a text file with the end point coordinates of each individual link for the most recently calculated route. This data only provides node coordinates and does not provide the shape 
coordinates for the links. Information on this screen is used to draw the straight-line segments on the Route Map screen discussed in Sect. 4.8.3.1. Most users will not have a need to view this data.

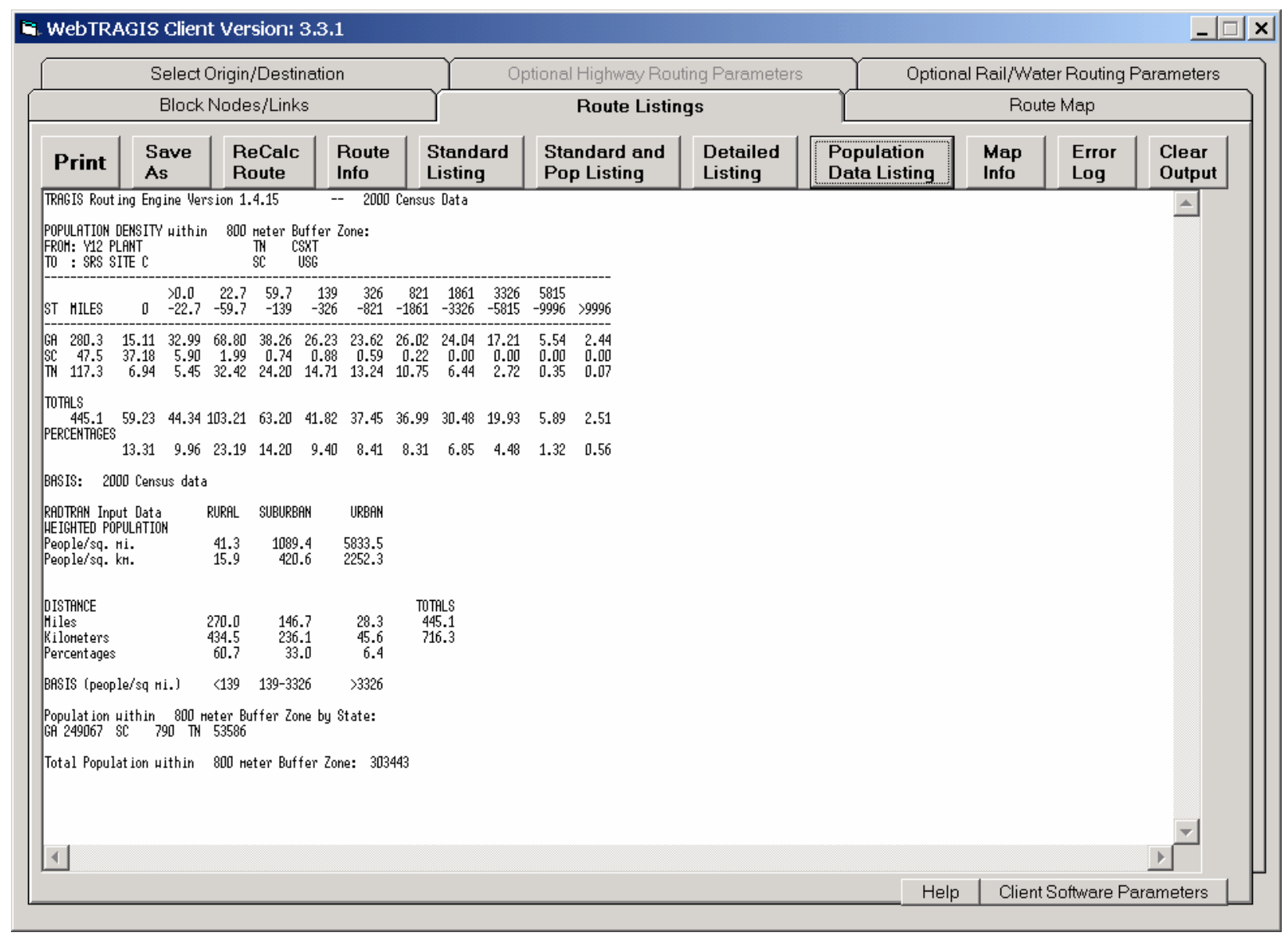

Fig. 4.7. Example of the population data listing for a rail route.

\subsubsection{Error Log}

If the model generates an error, it will automatically be displayed in the Route Listings screen. The Error Log button can be used to redisplay any error messages that the model generates on the server. If the error message does not provide sufficient assistance to remedy the problem, please contact one of the TRAGIS development staff regarding the error message. Telephone numbers for the TRAGIS development staff can be determined by clicking on the Help button on the lower edge of any WebTRAGIS screen.

\subsubsection{Clear Output}

The Clear Output button will remove the contents of all the WebTRAGIS output files (the route info, standard listing, detailed listing, population data listing, map info, and error log files) that can be viewed in the Route Listings screen. The output files for WebTRAGIS are downloaded from the server to the client computer following the calculation of each route and overwrite earlier copies. This button provides a means to delete the output files on the client computer. 


\subsection{OPTIONAL RAIL/WATER ROUTING PARAMETERS SCREEN}

The Optional Rail/Water Routing Parameters screen provides access to a number of parameters that control various aspects of WebTRAGIS rail routing functions. When rail or water is the active mode, Optional Rail/Water Routing Parameters tab is available, and the Optional Highway Routing Parameters tab is greyed out and not available for selection. The Optional Rail/Water Routing Parameters screen only lists the rail option parameters when WebTRAGIS is in rail mode.

Selecting the Optional Rail/Water Routing Parameters tab will display a window similar to that shown in Fig. 4.8. This window identifies the additional options that can be altered. The various optional rail routing are discussed in the following sections.

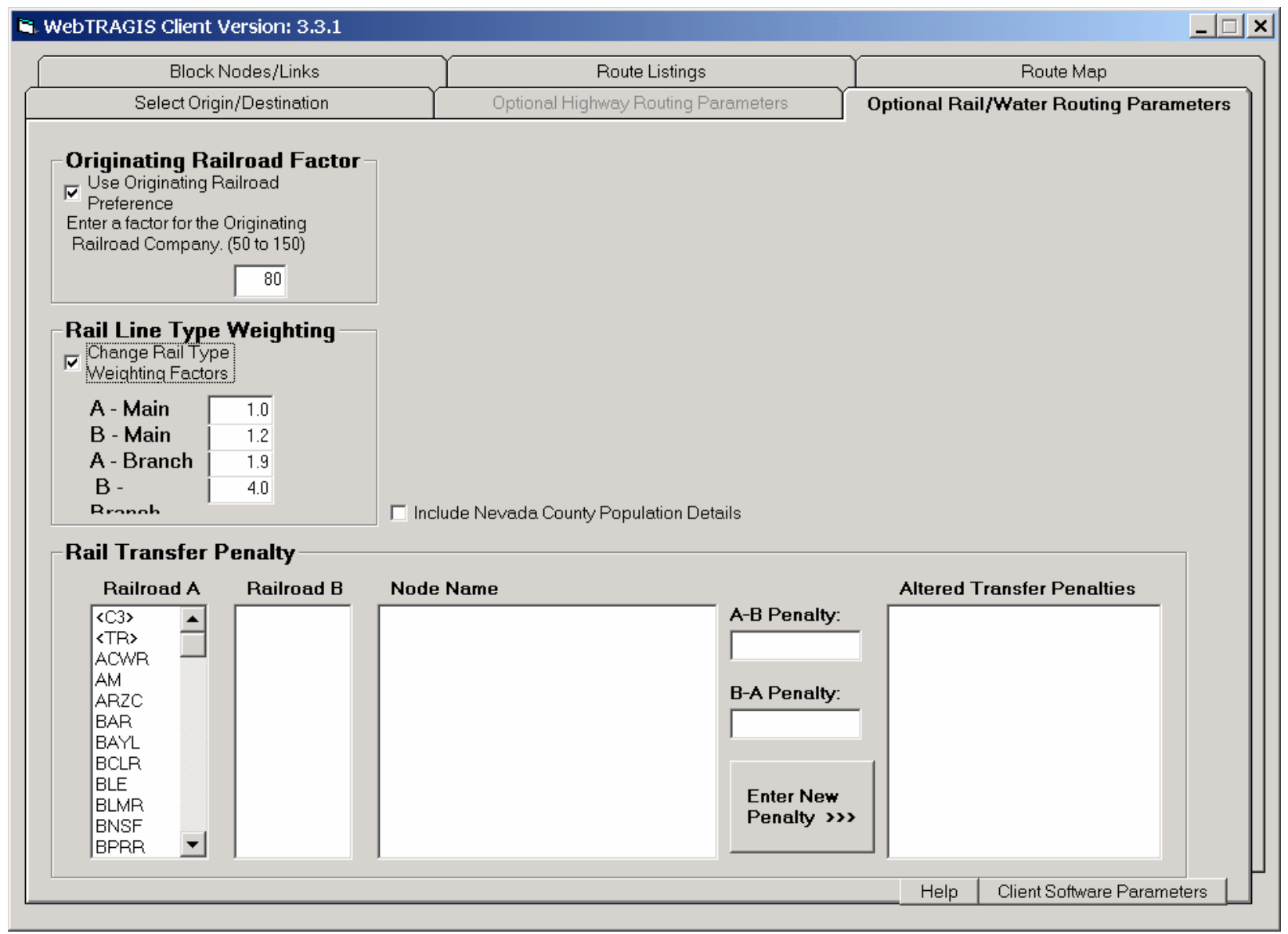

Fig. 4.8. Optional rail routing parameters.

\subsubsection{Originating Railroad Factor}

As discussed in Sect. 4.4.2.1, the commercial route type reduces the impedance of the originating railroad. By default, the use of the Originating Railroad Factor is in effect, as signified by the check mark and the value of 80 in the input box, as shown in Fig. 4.8. The impedance of the links on the originating railroad is multiplied by a factor of 0.80 . (This factor is entered as a whole number and then the model divides the value by 100.) WebTRAGIS allows this value to range between 50 and 150 . By entering a value over 100 , the result will be a disadvantage to the originating railroad and the model will attempt to move the shipment to another railroad. If the check is removed from the Originating Railroad 
Factor, the model will not change the link impedances for the originating railroad. The value of the Originating Railroad Factor is identified on the route listings unless this option is not activated.

\subsubsection{Rail Line Type Weighting}

The Rail Line Type Weighting factor option provides the capability to make adjustments to the mainline classification factors used to calculate routes. The use of these factors in the calculation of the route impedance is discussed in Sect. 4.4.2.1. Essentially, the distance of each link is multiplied by the respective mainline classification factor. For example, the model evaluates a ten-mile long A-mainline link as 10 units, while a ten-mile long B-branchline link is evaluated as 40 units. Because TRAGIS attempts to minimize the impedance between the origin and destination, the model attempts to maximize the use of A-mainline track.

Figure 4.8 shows this factor activated with the four different mainline classes and the default values. The weighting factors were determined after testing and examination of routing results and have been used in TRAGIS and the legacy INTERLINE model for many years. If this factor is not checked, these default values will be used in the determination of routes. Activation of this option allows the adjustment of the mainline classification factors. New values can be assigned by entering a different weight factors in the appropriate box. The route listing will note the new values if any of these values are adjusted.

\subsubsection{Rail Transfer Penalty}

The Rail Transfer Penalty feature permits the user to modify the transfer penalty between railroad systems in WebTRAGIS. The impedance to transfer between different rail systems varies with the type of transfer. In the TRAGIS 1:100,000-scale rail network, transfers are identified as primary, minor, terminal, and detour, with transfer impedances of $300,400,151$, and 1500 units, respectively. Thus passing through a primary transfer is equivalent to 300 miles of travel on A-mainline. Transfers between two major railroads through a terminal railroad, passing through two terminal transfers, will amount to 302 units, slightly more than a direct primary transfer. Minor transfers are low volume interchange locations between major railroads. The penalty for these less used transfers is 400 units. Finally, detour transfers are included in this new rail network. These transfers are not used for normal interchange traffic so they have a high penalty value of 1500 units.

WebTRAGIS provides the ability to view and modify the transfer penalty between rail systems. Figure 4.9 demonstrates how the Rail Transfer Penalty feature is used.

- The first step is to select a railroad from the Railroad A list. In this example the NS is selected.

- After this first railroad is selected, all the railroads transferring with the initially selected company are listed in the Railroad B list. In this example, the KCS is selected as the second railroad.

- Next, all transfer locations between the selected two railroads are listed in the Node Name window. If the window cannot display all the nodes, a scroll bar will appear on the right hand side of this window to allow the entire list to be viewed. In this example, ten interchange locations exist between the NS and KCS railroads.

- At this point, a single interchange location can be selected from the Node Name window by clicking on the name. As shown in Fig. 4.9, the Kansas City, MO, node is selected.

- After a node is selected, the existing transfer penalties will be listed in the A-B Penalty and B-A Penalty boxes. The transfer penalty will have the same value in both penalty boxes. The A-B Penalty is the transfer penalty from Railroad A to Railroad B, NS to KCS in this example. The B-A Penalty is the transfer penalty for the other direction KCS to NS. WebTRAGIS initially 
shows a transfer penalty of 300 units in both of the penalty boxes since this is a primary interchange. (This is not shown in Fig. 4.9 because new values have already been entered.)

- A new value can be entered into either of these boxes. In the example shown in Fig. 4.9, a penalty of 250 units is entered in the A-B Penalty box and a value of 200 units is entered in the B-A Penalty box.

- After entering new values, press the Enter New Penalty button and the new transfer penalties will be displayed in the Altered Transfer Penalties box. The Altered Transfer Penalties box lists the node number, first railroad abbreviation, second railroad abbreviation, and the new penalty value for the selected interchange location. In this example, the new penalty for transferring at Kansas City, Missouri, from the NS to the KCS is now 250 units and the transfer from the KCS to NS at the same location will only be 200 units (compared to the original penalty of 300 units, both directions).

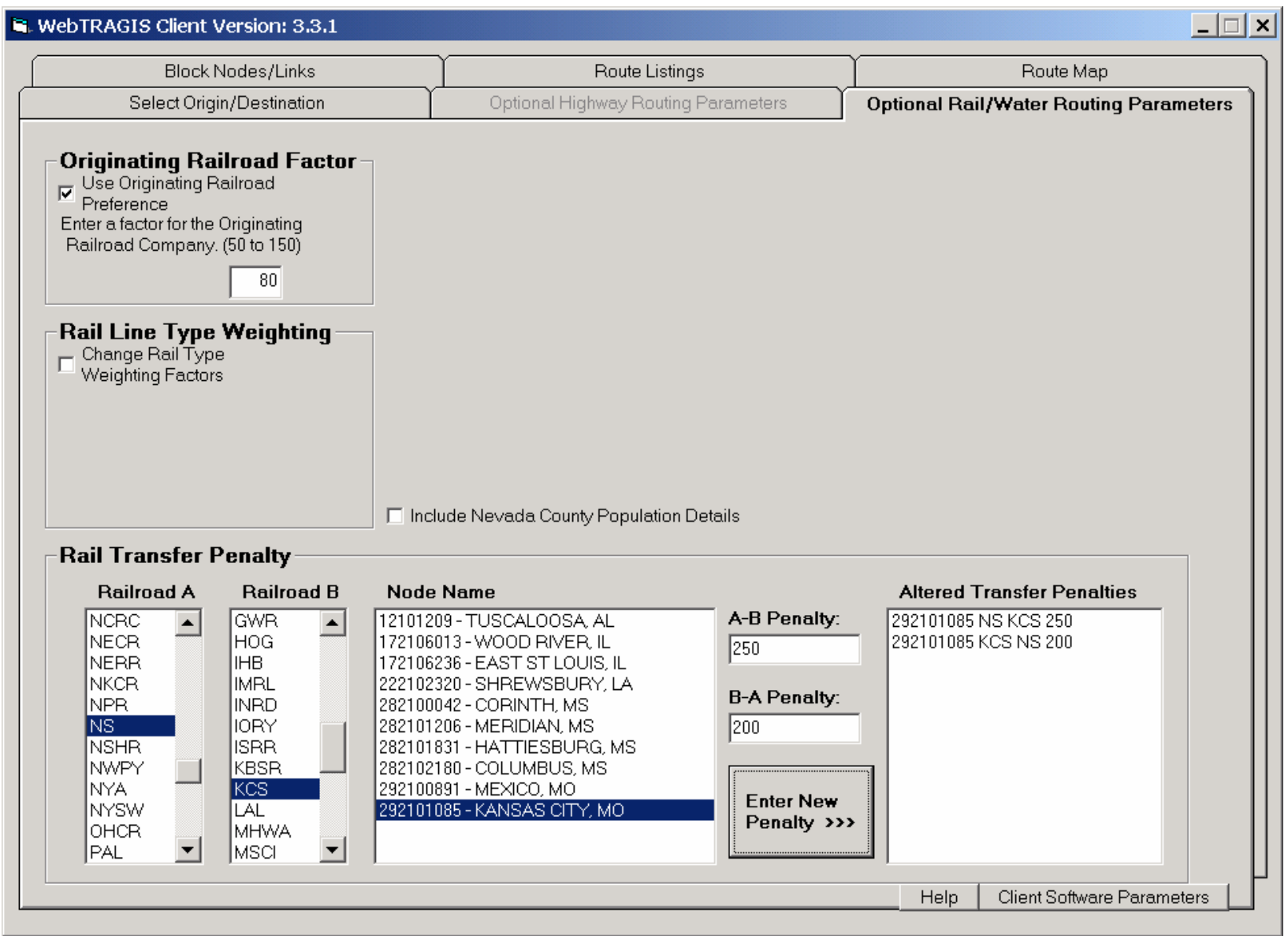

Fig. 4.9. Rail transfer penalty adjustment.

To remove an altered transfer penalty, double click on an entry in the Altered Transfer Penalties box and that item will be deleted.

WebTRAGIS only provides the capability to change individual transfer penalties currently. If all transfer penalties need to be adjusted, try running routes using the dedicated train route type discussed in Sect. 4.4.2.2. 


\subsubsection{Nevada County Population Details}

In the center of the Optional Rail/Water Routing Parameters screen is a check box called Include Nevada County Population Details. This parameter provides population density and count data at the county level for routes passing through Nevada. With the activation of this feature, a population density table and population counts for Nevada counties appears following the standard population data information. Figure 4.10 shows an example of a portion of the population density table with the Nevada county data. The Nevada population density table has a line of data for each county the route passes through. The first column of the table is the county code number. A key is provided at the end of the listing for the county names. To activate this option, click on this item to place a check mark in the box to the left of the option title.

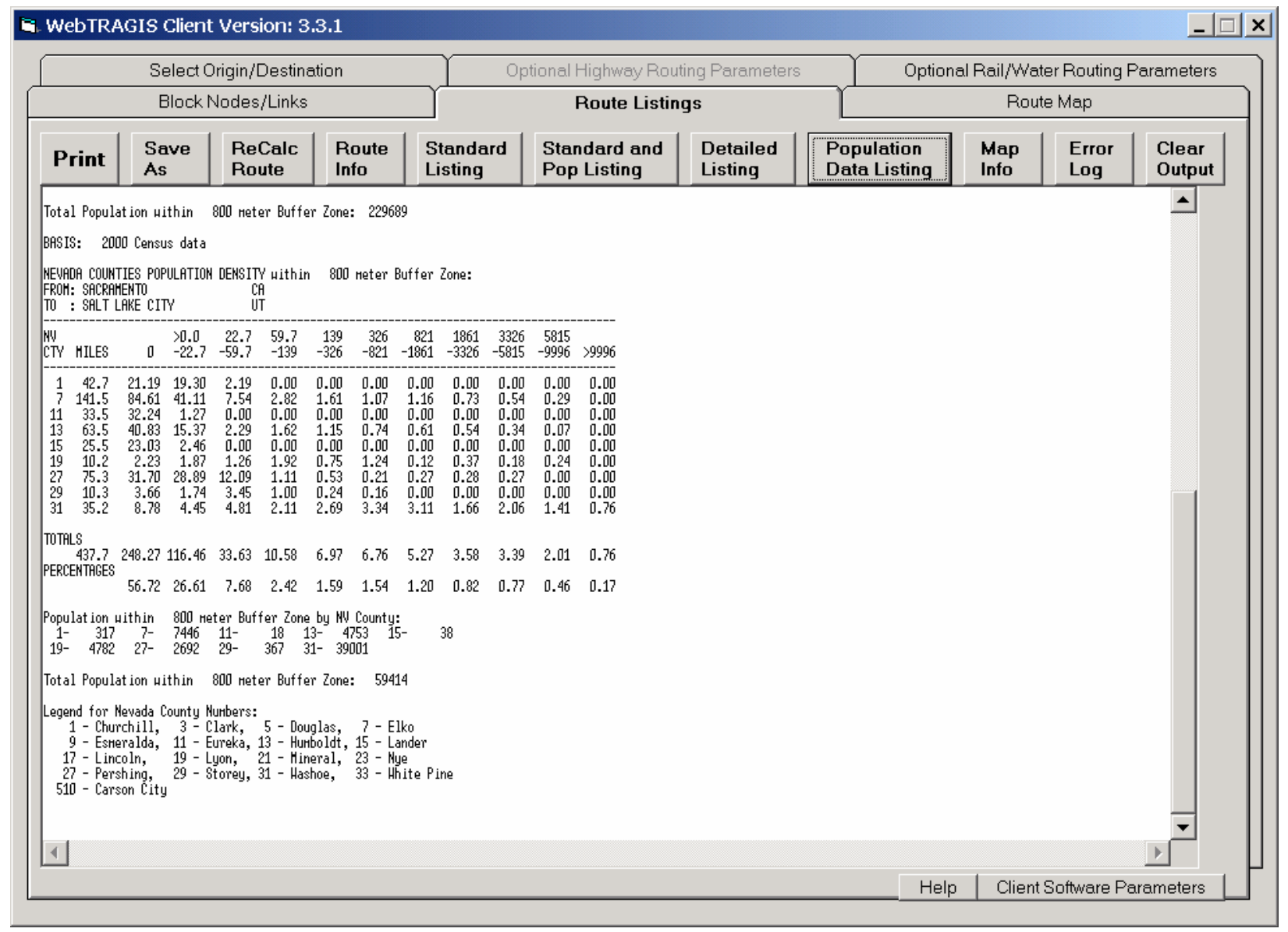

Fig. 4.10. Example of Nevada county population output for a rail route.

\subsection{BLOCK NODES/LINKS SCREEN}

The Block Nodes/Links option is recommended only for experienced users. This option allows the user to temporarily modify portions of the rail network database by blocking nodes, links, entire railroad companies, or states. This feature can be useful for analysis of various scenarios such as determining a route that avoids a temporarily damaged section of rail line due to weather conditions or derailment. The Block Nodes/Links screen is divided into four boxes, one for each function-block nodes, block links, block states, and block railroad company. The initial view of the window is shown in Fig. 4.11 below. Each of the four functions are discussed in the following sections. 


\subsubsection{Node Blocking}

Nodes can be blocked in the rail database thus making all the links that emanate from that node ineligible for routing. If a blocked node is on the only path between an origin and destination, TRAGIS will be unable to calculate a route. The Error Log feature on the Route Listing screen, discussed in Sect. 4.5.10, will display "ERROR: No path found. Exit Code: 21."

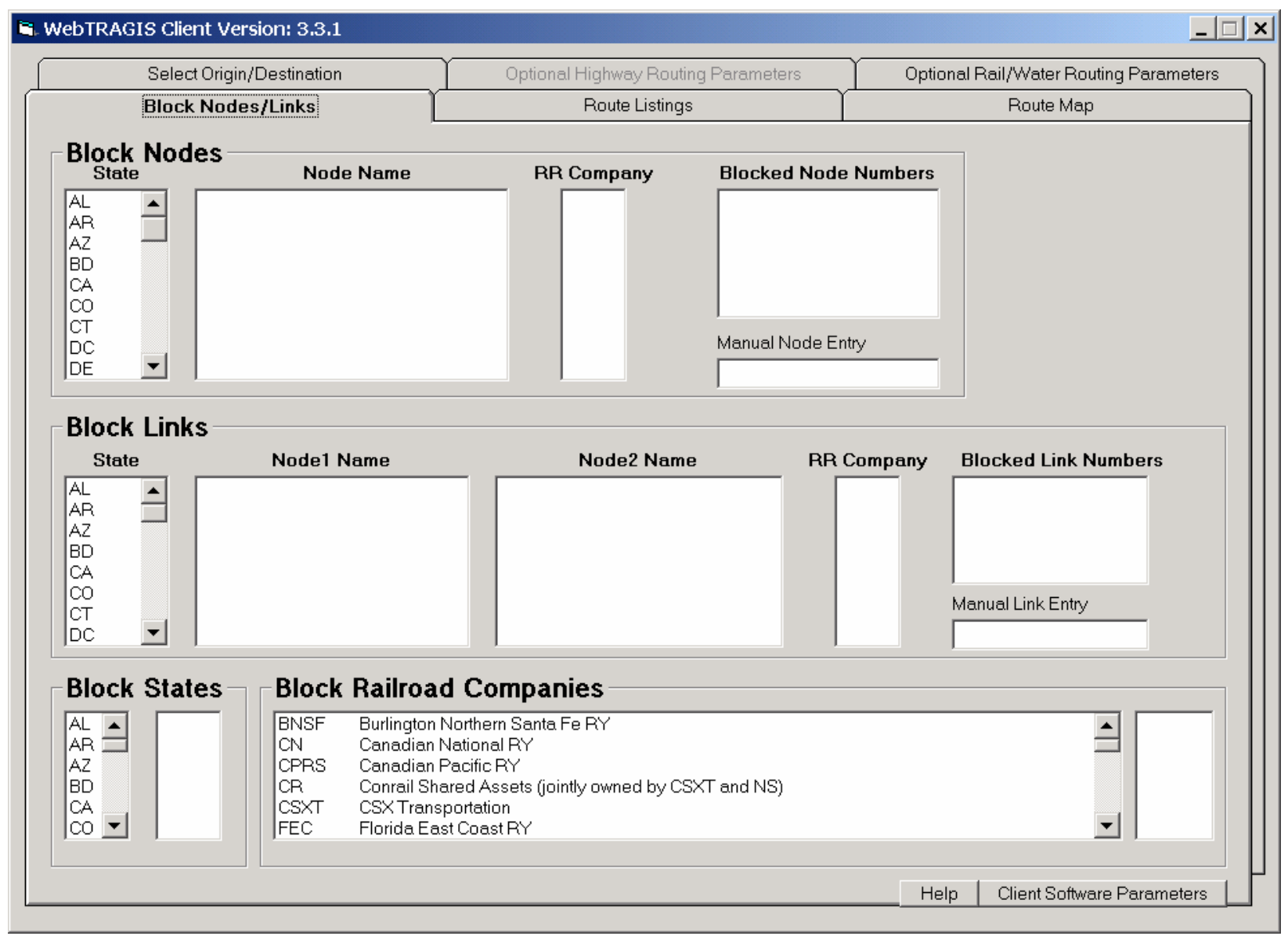

Fig. 4.11. Rail node/link blocking screen.

To block a rail node, go to the Block Nodes section on the Block Nodes/Links screen and scroll through the State list and click on the postal abbreviation of the desired state. After a state is selected, the Node Name list will display all named nodes for that state. Next scroll through the list and click on the node name to be blocked. A final step is required if more than one railroad is listed in the RR Company list. If only one railroad operates through the select node, the model will automatically add the selected node to the Blocked Node Numbers list. When multiple railroad companies are listed in the RR Company list, select the railroad companies that are to be blocked by clicking on the appropriate abbreviations. The node number, along with the railroad abbreviation is added to the Blocked Node Numbers list as each railroad company is selected. So if a particular node name has three railroad companies listed, three entries, one for each railroad, need to be listed in the Blocked Node Numbers list for that node to not be included on any possible route. 
Nodes exist in the rail network that will not appear in the Node Name list. Not every node has a name and some nodes may have non-unique names. Such nodes can be blocked, but the node number and railroad abbreviation have to be determined and manually entered in the Manual Node Entry box below the Blocked Node Numbers list. A node number and railroads can be determined by using the MapTips function (see Sect. 4.8.2) or the Identify button (see Sect. 6.1.14 or discussion in Sect. 4.4.1.2) on the Route Map screen. When entering an unnamed node number, enter the node number followed by a space and the railroad abbreviation. The node number and railroad abbreviation is added to the Blocked Node Numbers list after the enter key is pressed.

Figure 4.12 shows an example of blocking two nodes near downtown Knoxville, Tennessee. The K\&A Junction node is selected in the Node Name list. This node is served by two railroad systems - GFRR and NS. Only the NS use of the K\&A Junction node has been selected and blocked. (Please note that the GFRR could still operate through this node.) This example also demonstrates the manual entry of node 472101006, which is one of three nodes named Knoxville, shown in Fig. 4.3. (K\&A Junction is just southwest of the 472101006 node.) Figure 4.13 shows the effects of the blocking of these two nodes of a NS rail route between the Sevier Yard, just northeast of Knoxville, and Citico Junction in Chattanooga.

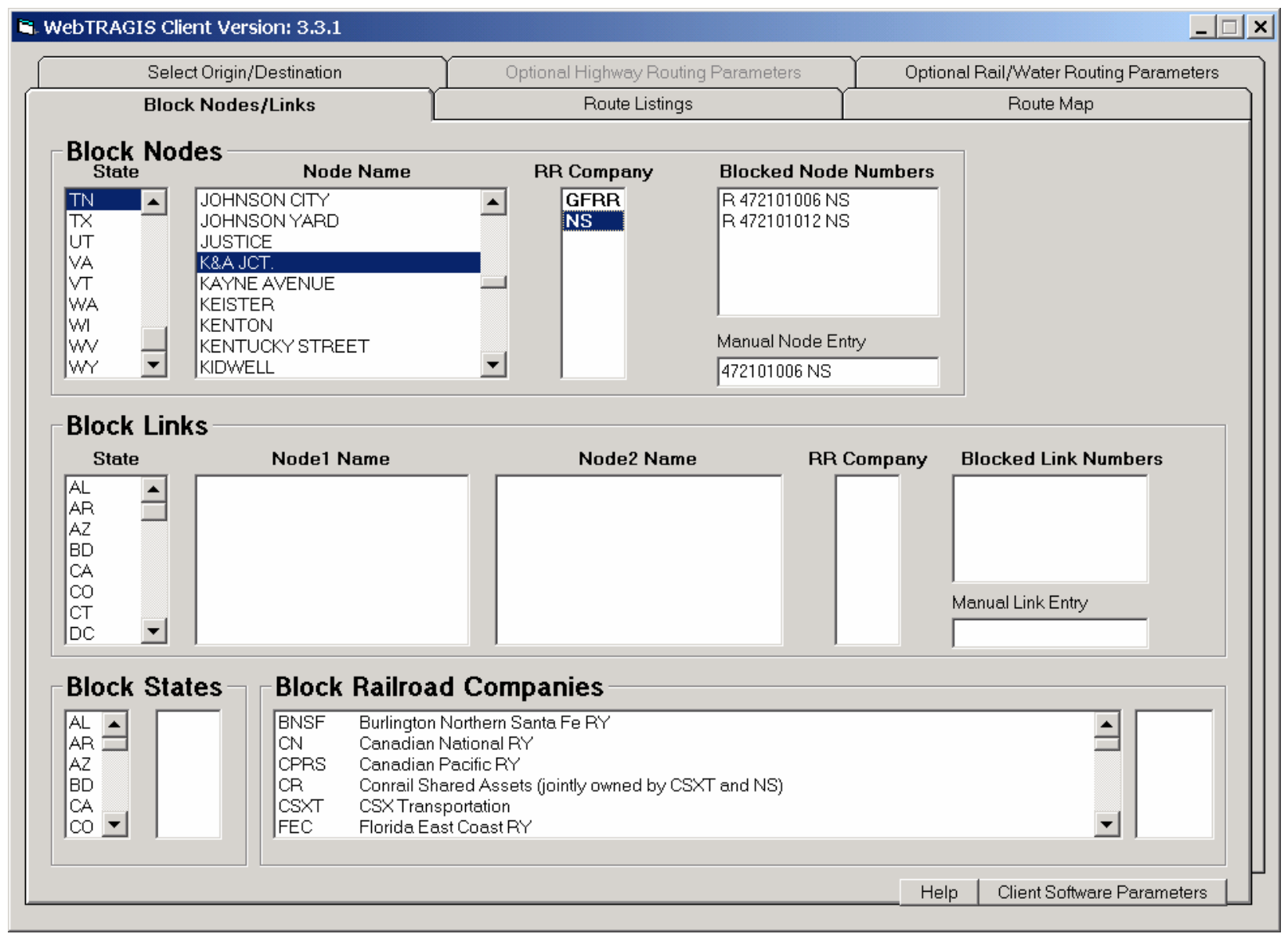

Fig. 4.12. Rail node blocking

A node number may be removed from the Blocked Node Numbers list by double-clicking on it. The Blocked Node Numbers list does not provide the name of blocked nodes, but the route listing does provide both the node number and name of blocked nodes. 


\subsubsection{Link Blocking}

Links in the rail network can be modified to temporarily remove them from consideration on a route. Similar to node blocking, a link can be blocked by two methods - selection from the list box or manual entry of a link number. As with node blocking, if a blocked link occurs on the only possible route between the origin and destination, the model will not be able to determine a path. An error message will be displayed in the Route Listings screen indicating that no path could be found. Figure 4.14 shows an example of blocking links by both methods.

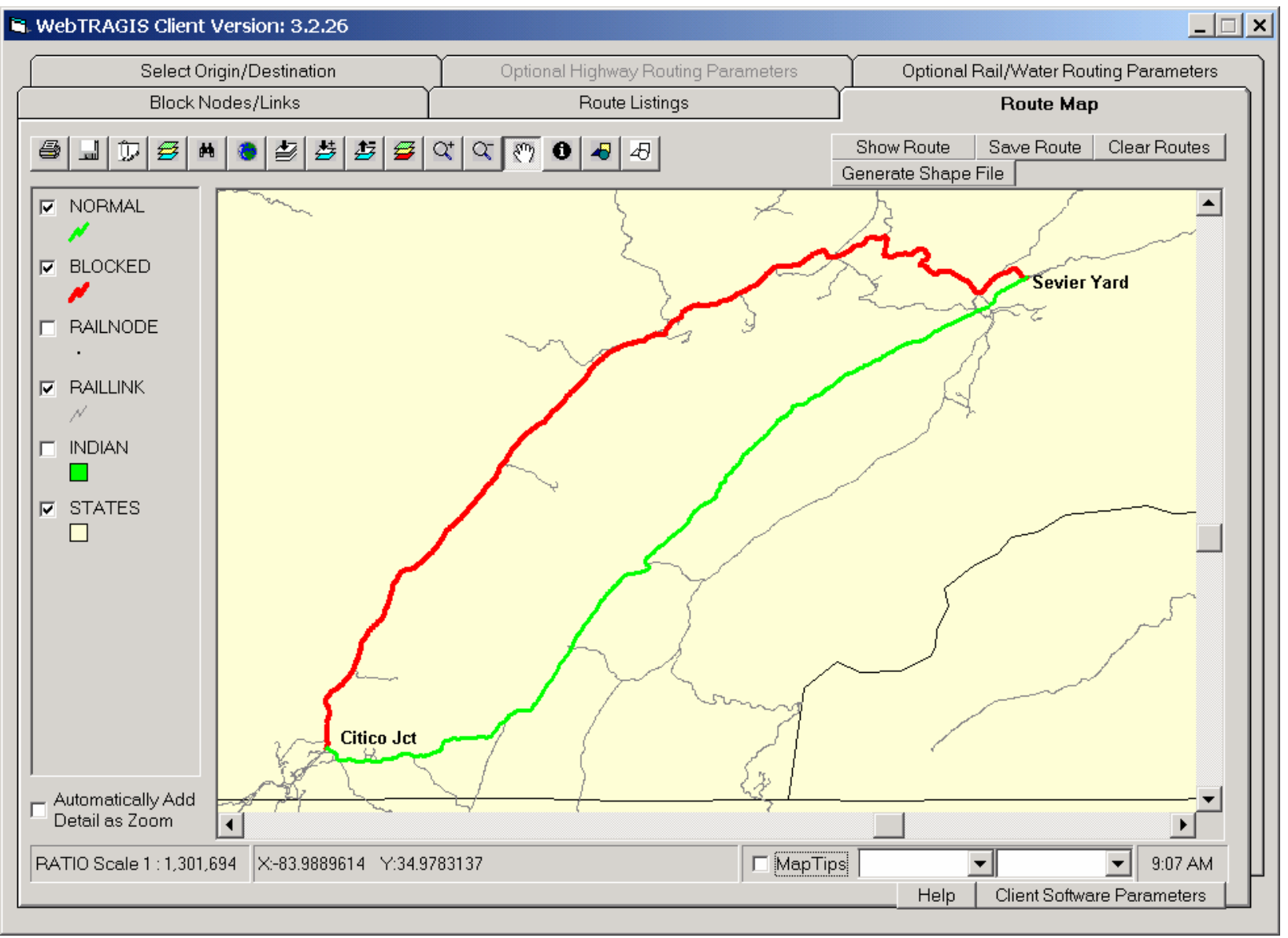

Fig. 4.13. Example of different rail routes with blocking nodes.

\subsubsection{Primary method}

The primary method of blocking a link involves identifying the link by the two end node names. Since not every node in the rail network is named, only those links with named nodes at both ends can be blocked with this method.

The first step is to select the state by scrolling through the State list and clicking on the desired postal abbreviation. Following the state selection, the Node1 Name list is populated with all the node names in this state. Select the desired node by clicking on the name. After this selection, the Node2 Name list identifies the names of all the nodes connected to the highlighted location in the Node1 Name list. (If no names are displayed, this indicates that all the links connecting to the name selected in the Node1 Name list have either unnamed nodes or non unique node names. Use the alternative method, discussed in the 
next section, to block such links.) Select the appropriate location from the Node2 Name list by clicking on the name. Finally, the RR Company list is populated with all the railroads that operate on this link. If only one railroad is shown, the link number and railroad will be displayed in the Blocked Link Numbers list. But if multiple railroads operate on the link the final step is to select which railroad companies should be blocked on the link. Clicking on the appropriate abbreviation blocks traffic for the selected railroad on this link. When blocking a link with multiple railroads, if only one railroad is blocked, the other railroads will be able to operate on the link.

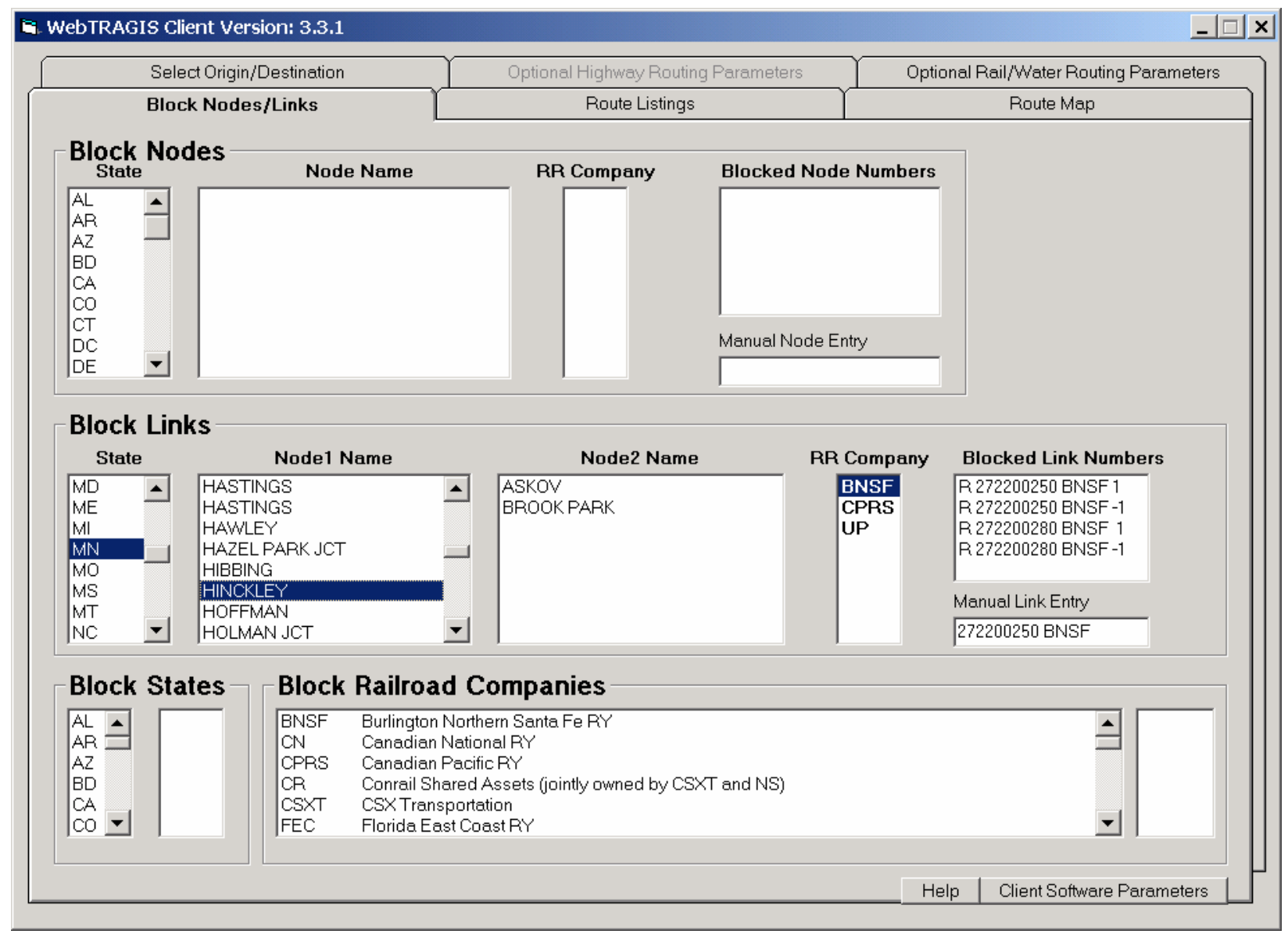

Fig. 4.14. Rail link blocking.

For each railroad selected, two entries appear in the Blocked Link Numbers list. Each entry lists the same link number and railroad abbreviation, but the first entry ends with a ' 1 ' and the second entry with a ' -1 '. Links are represented in the model as one-way connections. The ' 1 ' link extends from the from-node to the to-node. Conversely, the '-1' link extends the other direction, from the to-node to the from-node. The best way to determine the direction of a link is to block the link and then calculate a route. The route listing identifies all blocked links and identifies the ' 1 ' and ' -1 ' link. To block a link for only one direction, double click on the appropriate entry in the Blocked Link Numbers list and it will be removed from the list.

In the example shown in Fig. 4.14, BNSF service over the link between Hinckley and Askov, Minnesota is blocked. This is link number 272200280. Two other railroads (CPRS and UP) operate over this link, but are not blocked. Traffic by CPRS and UP can still operate over this link. Note that three node names are listed in the Node2 Name box in Fig. 4.14 as connecting to the Hinckley node. Figure 4.14 show 
three links connecting to the Hinckley node - the Askov node, a node to the southwest (Brook Park), and a node to the south. Figure 4.15 shows the normal route (the green route on the map) between Duluth and Minneapolis, Minnesota, that TRAGIS calculated before this link is blocked. The blocked route (the red route on the map) shows the result of blocking this link. The end points of this link (Askov and Hinckley) are identified on the map. Figure 4.16 shows the route listing for the example discussed. Links that are blocked are identified near the top of the listing. For this example, the from-node on the link 272200280 is Askov and the to-node is Hinckley.

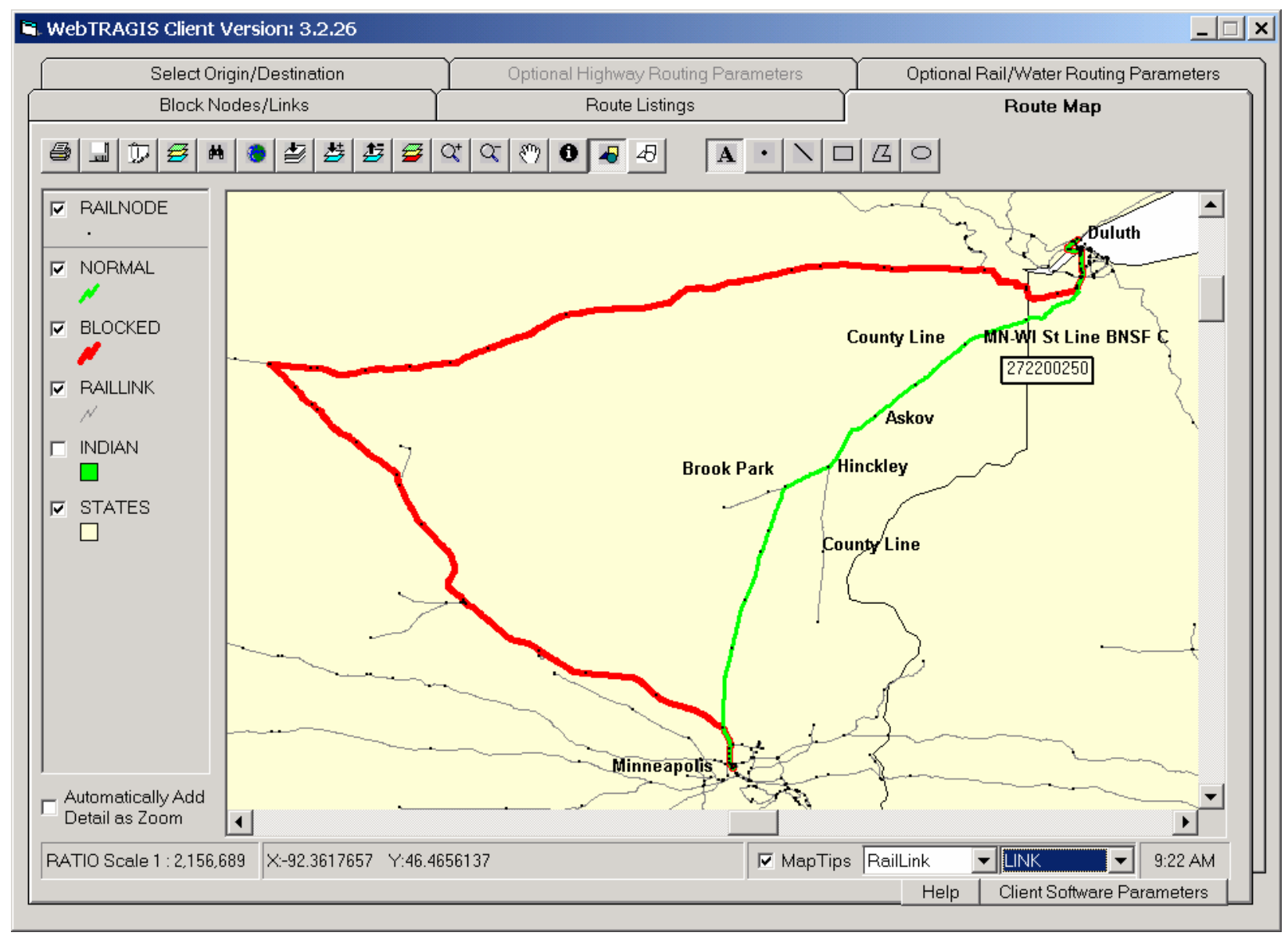

Fig. 4.15. Example of different rail routes with blocked links.

\subsubsection{Alternative method}

A number of links in the rail network cannot be selected for blocking with the primary method discussed in the previous section. Some nodes are unnamed and other nodes have non-unique names. An example of non-unique named nodes is nodes at county boundaries. These nodes are all named "County Line." If one end point of a link is named, but the other is not, the primary method is unable to select that link. The only way to block such links is to manually enter the link number in the Manual Link Entry box that is located below the Blocked Link Numbers box. When entering the link number, a railroad company must be also provided. At least one space should separate the link number and railroad abbreviation.

An example of a manually entered link number is shown in Fig. 4.14. Link number 272200250 with BNSF operation is entered in the Manual Link Entry box. After entering this data in the Manual Link 
Entry box, press the enter key and two records for this link appear in the Blocked Link Numbers box, one for each direction.

To determine a specific link number, go the Route Map screen and activate the MapTips function, as shown on Fig. 4.15. Select RailLink in the first pull-down box and Link in the right pull-down box. Next move the pointer over the desired link and the link number will be displayed. Figure 4.15 shows a link number below the link. (The pointer does not appear on this captured screen image.) The eastern node of this link is on the Minnesota-Wisconsin state line and the western node is a county line node. Figure 4.16 shows this blocked link as the first two records in the list of blocked links. The "1" or positive direction of link R272200250 goes from the Minnesota-Wisconsin state line to the county line node in Minnesota. Alternatively, the "-1" or negative direction entry for this link proceeds from the county line node in Minnesota to the Minnesota-Wisconsin state border node.

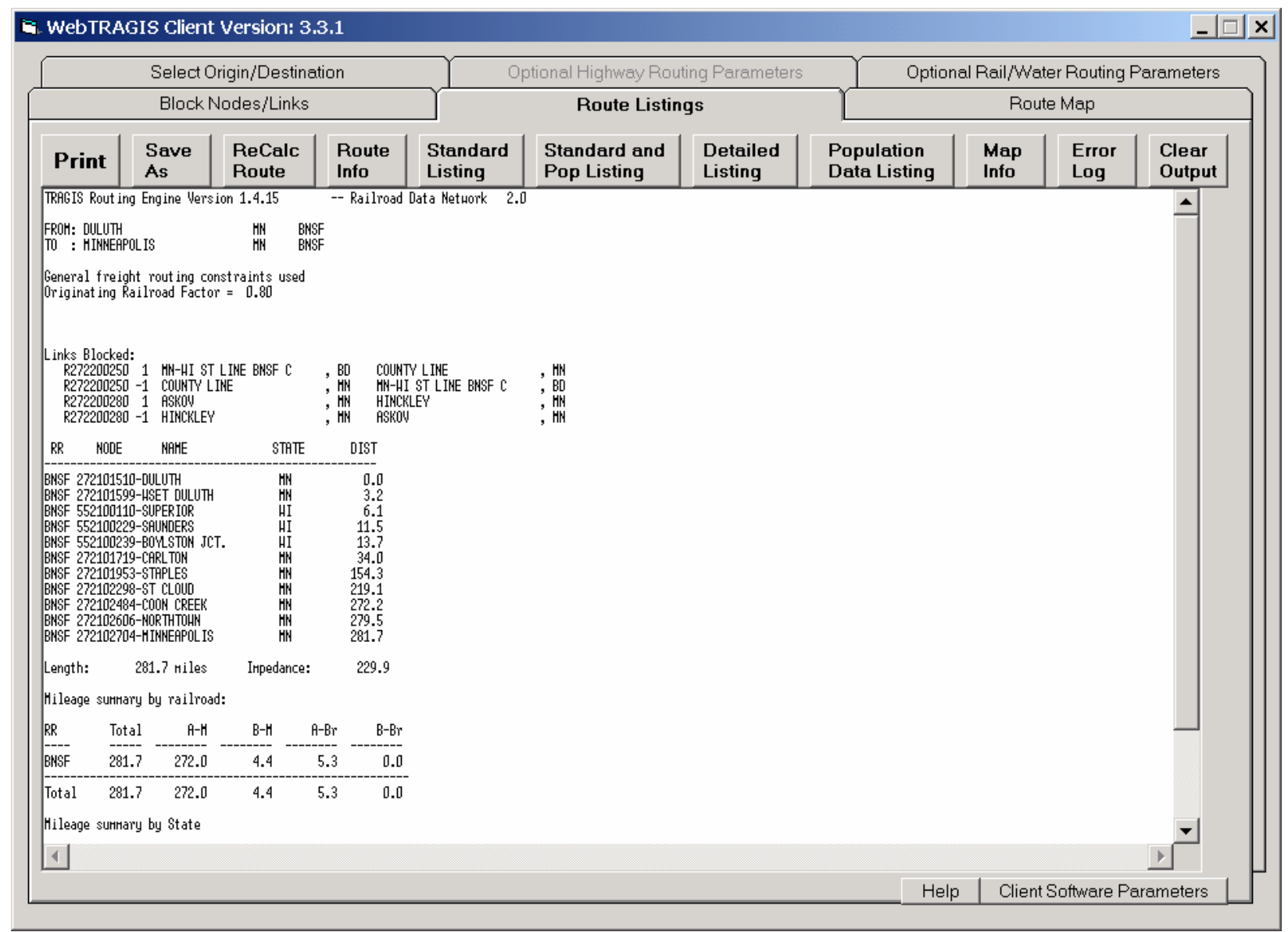

Fig. 4.16. Route listing of rail route with blocked links.

\subsubsection{Block States}

An entire state can be temporarily removed from the rail network database by selecting the state (or states) from the Block States list in the lower left corner of the Block Nodes/Links screen. Scroll through the list of states and click on the desired abbreviation to block a state. The blocked states will appear in the right hand list in this portion of the screen. A state may be restored from the removal list by doubleclicking on the state abbreviation in the right hand box. If multiple states are blocked, each entry needs to 
be restored separately. The route listings identifies any blocked states in effect on the description of parameters used to calculate routes.

Figure 4.17 shows the blocking of the state of Delaware. Note that after Delaware is blocked, its abbreviation is removed from the left hand list of states and placed in the blocked list to the right. Figure 4.18 demonstrates the effect of the blocking of the state of Delaware. A normal rail route on the CSXT system between Richmond, Virginia, and Philadelphia, Pennsylvania, is shown on this figure with the green line. The red route shows the effect of blocking Delaware. This blocked route takes a dramatically different and longer route, primarily because this route remains on the CSXT system for the entire shipment.

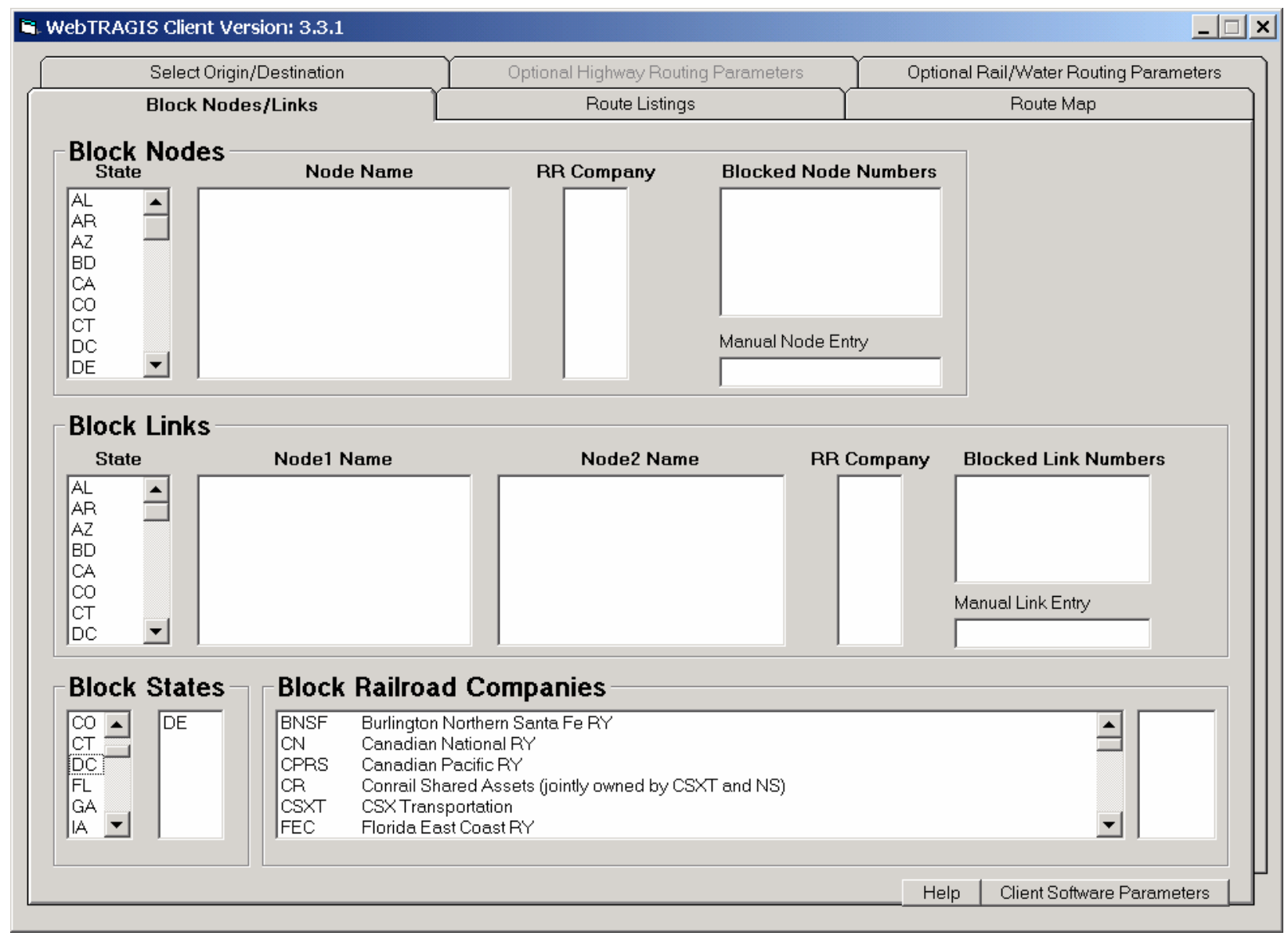

Fig. 4.17. Blocking a state for a rail route.

Care should be taken with blocking states with rail routing as the routing results may change dramatically. The connectivity of the rail network is considerably different compared to the highway network. Blocking a state removes that state from consideration and can result in significantly different routes.

\subsubsection{Block Railroad Companies}

The Block Railroad Companies feature provides a method to temporarily remove an entire railroad system from consideration. This capability is quite powerful and should be used with care. 
The TRAGIS rail network consists of 97 rail company groups. These company groups are listed in the scroll box in three different sets, each in alphabetical order. The first nine entries in this list (BNSF through UP) are the larger railroad systems in the country. Following this set (ACWR through WSOR) are the smaller rail systems. The final four rail systems $(<\mathrm{C} 3>$, $<\mathrm{TR}>$, AMTK, and $<\mathrm{OR}>)$ are special systems and generally will not need to be blocked. A complete list of railroad companies and their abbreviations can be found in Appendix B.

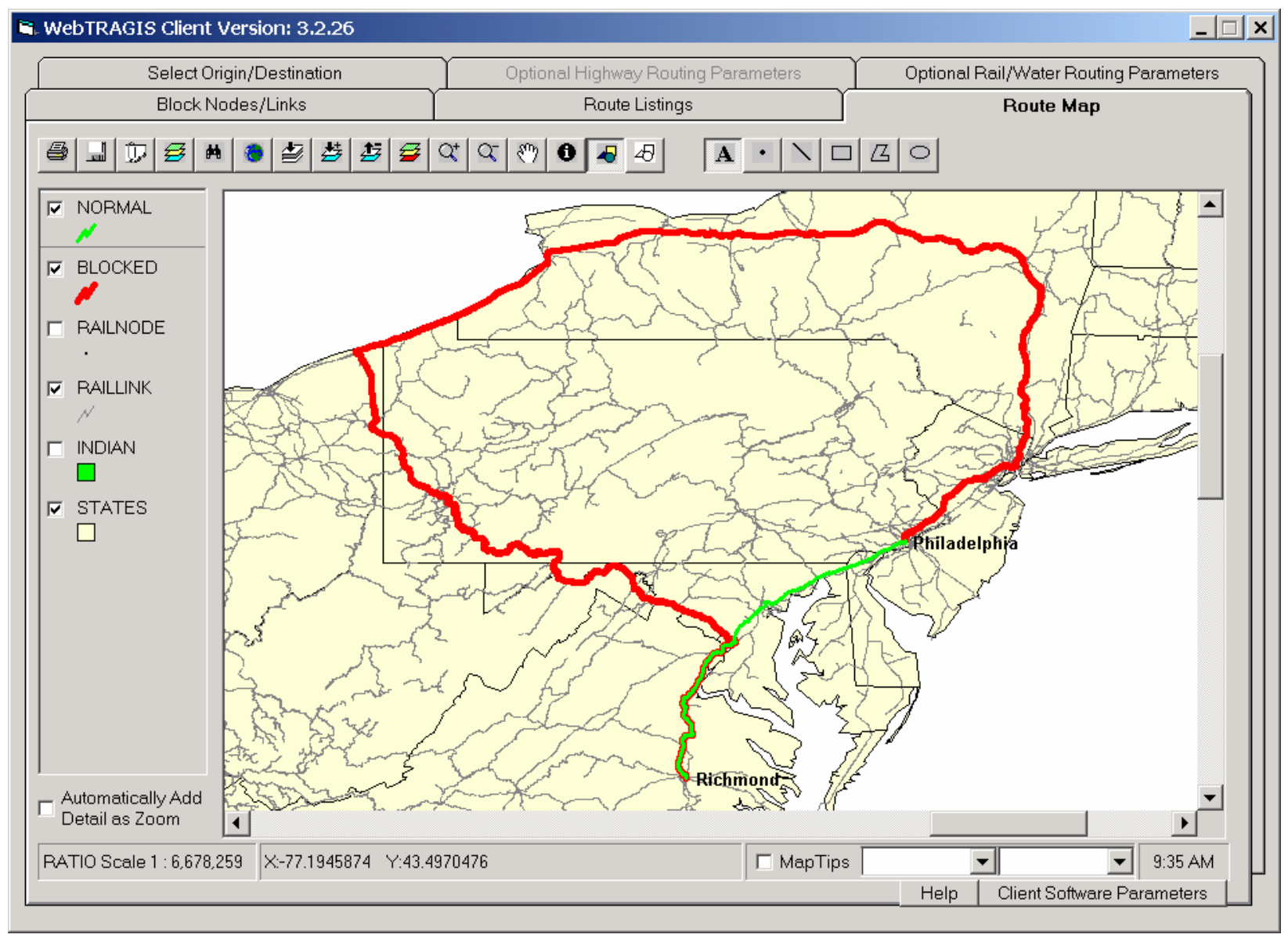

Fig. 4.18. Example of a rail route with a state blocked.

To block railroad companies, go to the Block Nodes/Links screen. In the lower portion of the screen is the Block Railroad Companies box. To remove a rail system from consideration, scroll through the list to locate the desired railroad and click on the name to block it. The abbreviation will appear in the box to the right indicating that this railroad has been blocked. The route listings identify the rail systems removed from consideration. To restore a blocked railroad system, double-click the abbreviation in the right hand box. If multiple rail systems are blocked, each entry needs to be restored separately.

Figure 4.19 shows the Montana Rail Link (MRL) selected and blocked. The box to the right of the list of railroad companies confirms that the MRL is blocked. Figure 4.20 demonstrates the effect of blocking this railroad. The unblocked or normal route between Casper, Wyoming, and Pasco, Washington, is shown as the green route on this figure. This route originates and terminates on the BNSF, but uses the MRL between Laurel, Montana, and Spokane, Washington. (A predecessor of BNSF formerly owned the MRL line. Since the formation of the MRL, BNSF has routed traffic over the MRL.) When the MRL 
system is blocked, the model generates the blocked route shown as the red line on Fig. 4.20. This route remains on the BNSF system and follows a more northerly path.

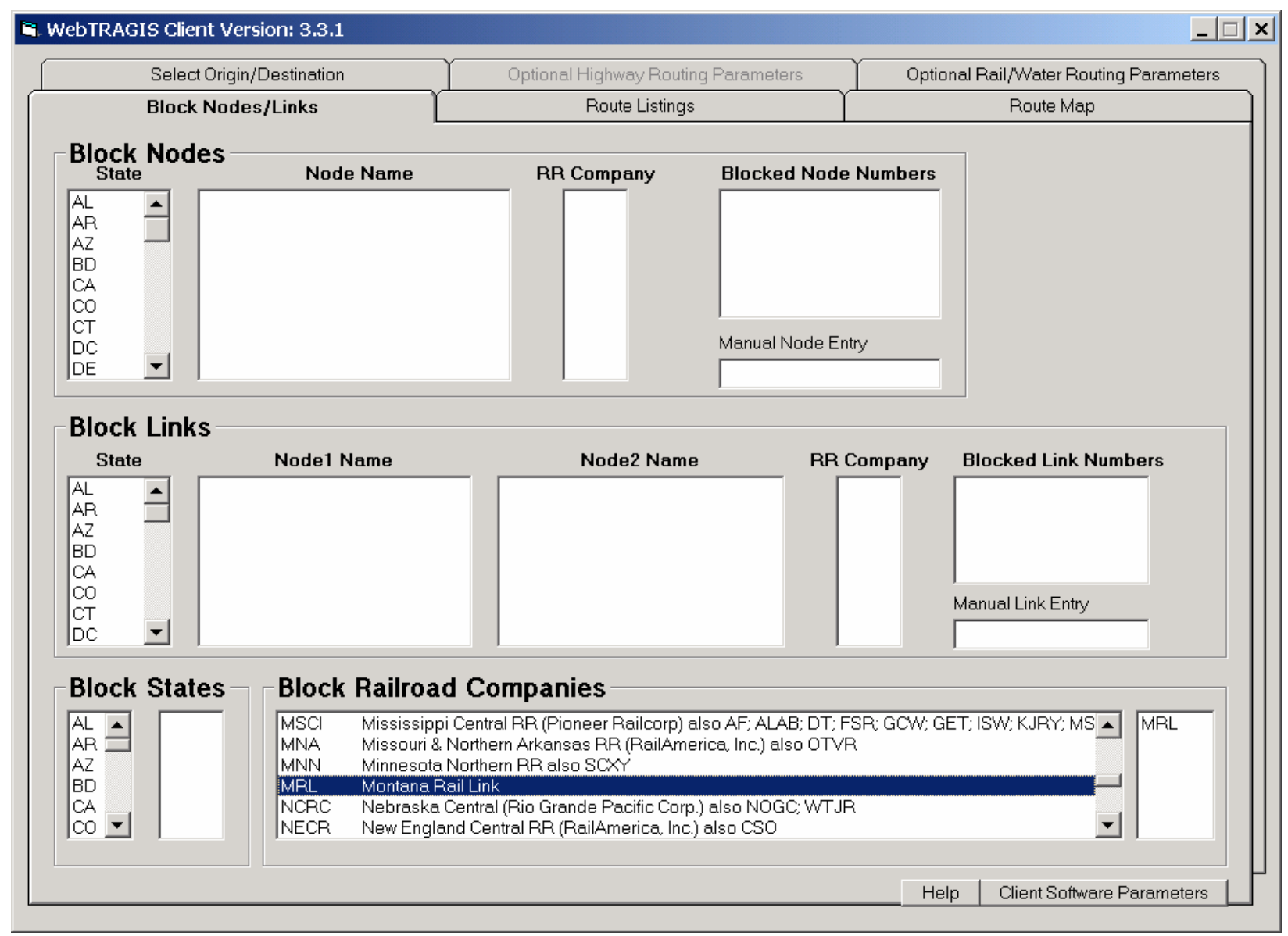

Fig. 4.19. Blocking a railroad company.

\subsection{ROUTE MAP SCREEN}

Figure 4.21 shows the Route Map screen for the rail portion of WebTRAGIS. This section describes the functionality specific to the rail portion of the model. Section 6 of this user's manual provides a detailed description of common features to the highway, rail, and waterway Route Map screens.

\subsubsection{Rail Coverages}

The Route Map screen consists of the primary window displaying a map of the continental U.S. and a legend area on the left hand portion of the screen. Four coverages are shown in the legend-Railnode, Raillink, Indian, and States. The Railnode coverage consists of points representing each node in the rail network database. The rail network is shown as one coverage, called Raillink. As with the highway transportation mode, the Indian and State coverages show Native American tribal lands and the state boundaries. All four coverages are initially displayed on the screen. By clicking on the check box, the check mark can be removed, which caused the display to be redrawn without that coverage shown on the map. 


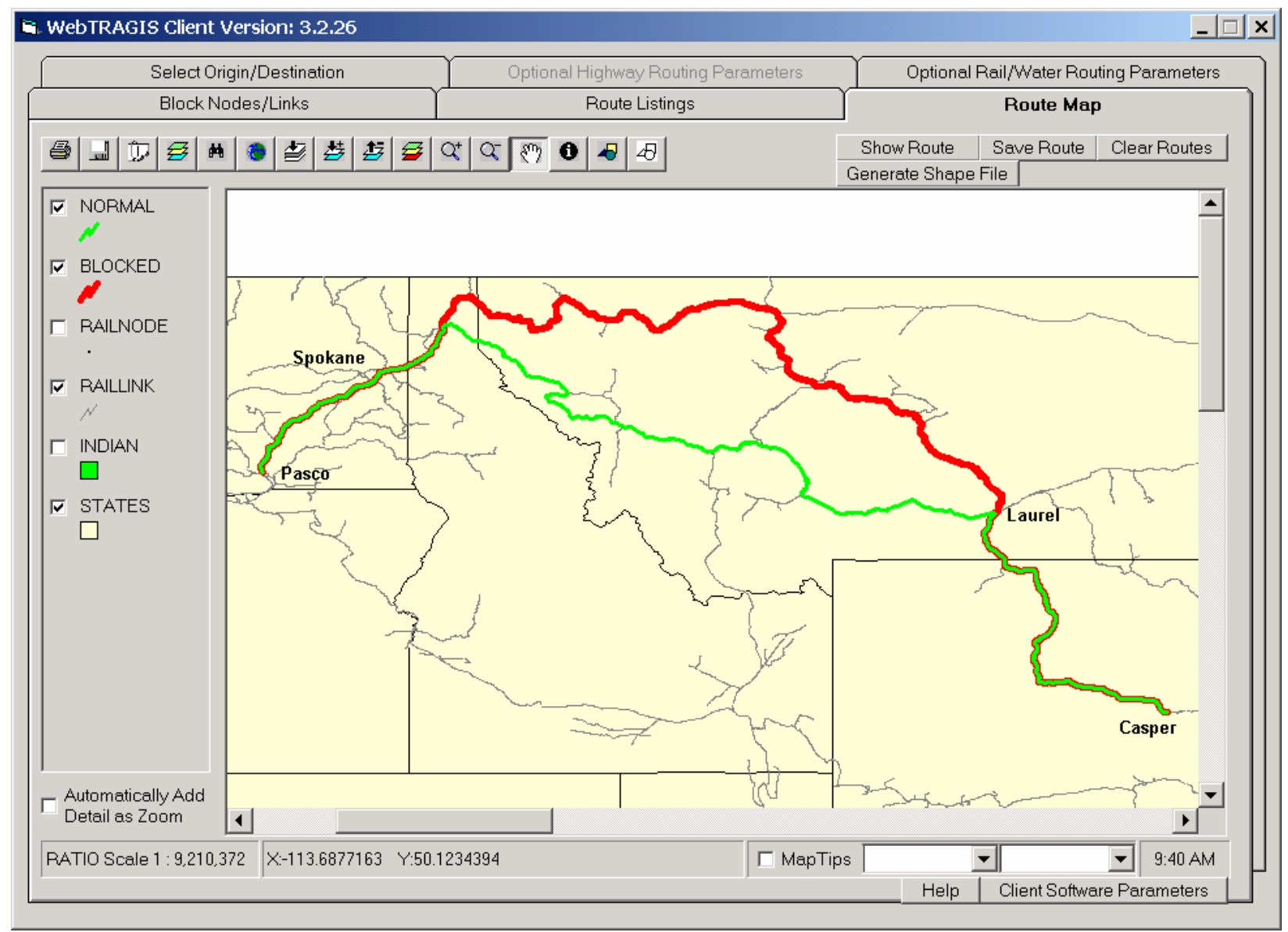

Fig. 4.20. Example of a rail route with a railroad company blocked.

\subsubsection{Using MapTips}

In the lower right center of the screen, MapTips has been checked, as shown in Fig. 4.21. (The MapTips item is checked for this example to assist the discussion of this feature. By default, MapTips is not checked when the Route Map screen is displayed.) Two pull down boxes appear to the right of the MapTips label. The left pull down box is used to select the coverage, in this case the Railnode coverage. The right pull down box is used to select an individual attribute of coverage. With the Railnode coverage is selected, the attribute field defaults to Name. Zooming into a specific area best uses the MapTips feature. Move the pointing device over a node and the name of the node will be shown on the screen. MapTips can be used to examine the attributes for any of the coverages shown on the Route Map screen.

\subsubsection{Displaying Routes}

WebTRAGIS provides two methods of displaying a calculated route in the Route Map screen. The first is a simple and fast method that draws a stick figure representation of the route. The second takes more time, but displays the complete shape of the route. Figure 4.22 provides an example of the difference between the two methods. The route shown in this figure is the NS line passing northeast of the Great Smoky Mountains along the North Carolina-Tennessee border. The green segments show the route representation using the stick figure method and the blue line is the shape file method. The red circles show the rail network nodes. 


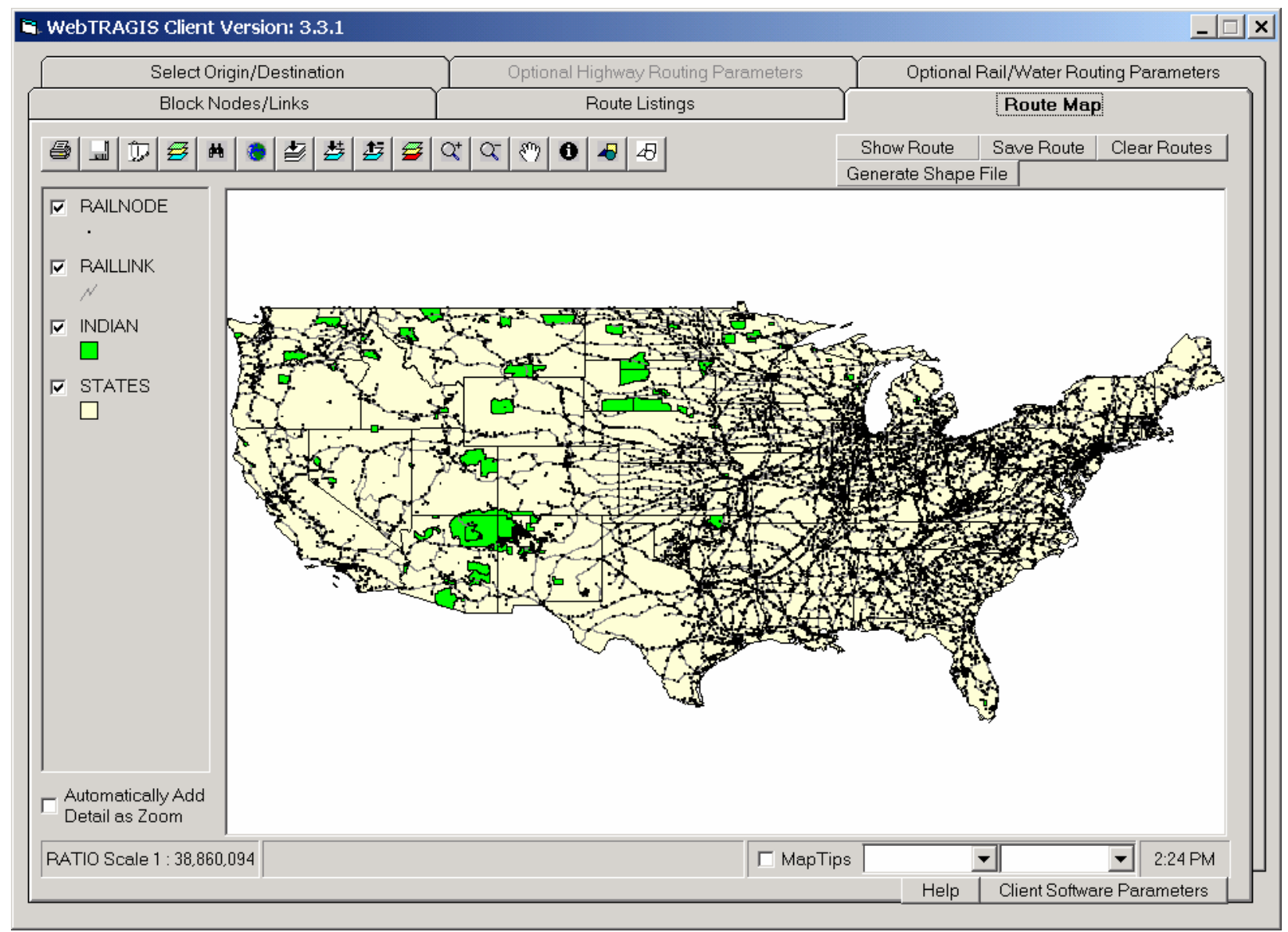

Fig. 4.21. Rail route map screen.

\subsubsection{Stick figure routes}

The simplest method draws straight-line segments between nodes. This technique is very fast and is sufficient for first draft national scale maps. The top row of buttons on the upper right hand portion of the Route Map screen is used to control the drawing of routes with this method. The Show Route button will display the most recently calculated route. (Even if you have just started WebTRAGIS, the most recently calculated route means the last route you have created with the model.) If you are creating a series of routes, the Show Route button can be pressed after the calculation of each to display multiple routes on the screen. Each successive route is displayed in a different color on the screen. The Save Route button saves all the routes displayed on the screen in one file in the ESRI shape file format. When this button is pressed, a save as window is opened to allow the user to select the folder the file is stored. The Clear Routes button will clear all routes displayed on the screen.

\subsubsection{Shape file routes}

The Generate Shape File button provides the capability to make a map of the route showing the full detail of the geographic shape available with the 1:100,000-scale rail network available in WebTRAGIS. This process is slightly more complicated compared to the method described in the previous section, but is the preferred method if large scale (showing small areas) maps are needed. When the Generate Shape File button is pressed a Save As window is opened, which provides the method for a unique file name to be entered. The file is saved in the ESRI shape file format, which can be used with ESRI GIS software, 
such as ArcView and ArcGIS. After entering the file name, press the Save button. After a brief delay, the shape file will be created, displayed on the map, and an entry will be added to the legend. Click on the name of this new coverage to change the attributes, such as color and line width, of the route. To remove a generated shape file from the Route Map screen, select the coverage (click on the coverage legend so it appears to be slightly raised or highlighted) and then press the Remove Active Map Layer button, which is on the button bar (see Sect. 6.1.9).

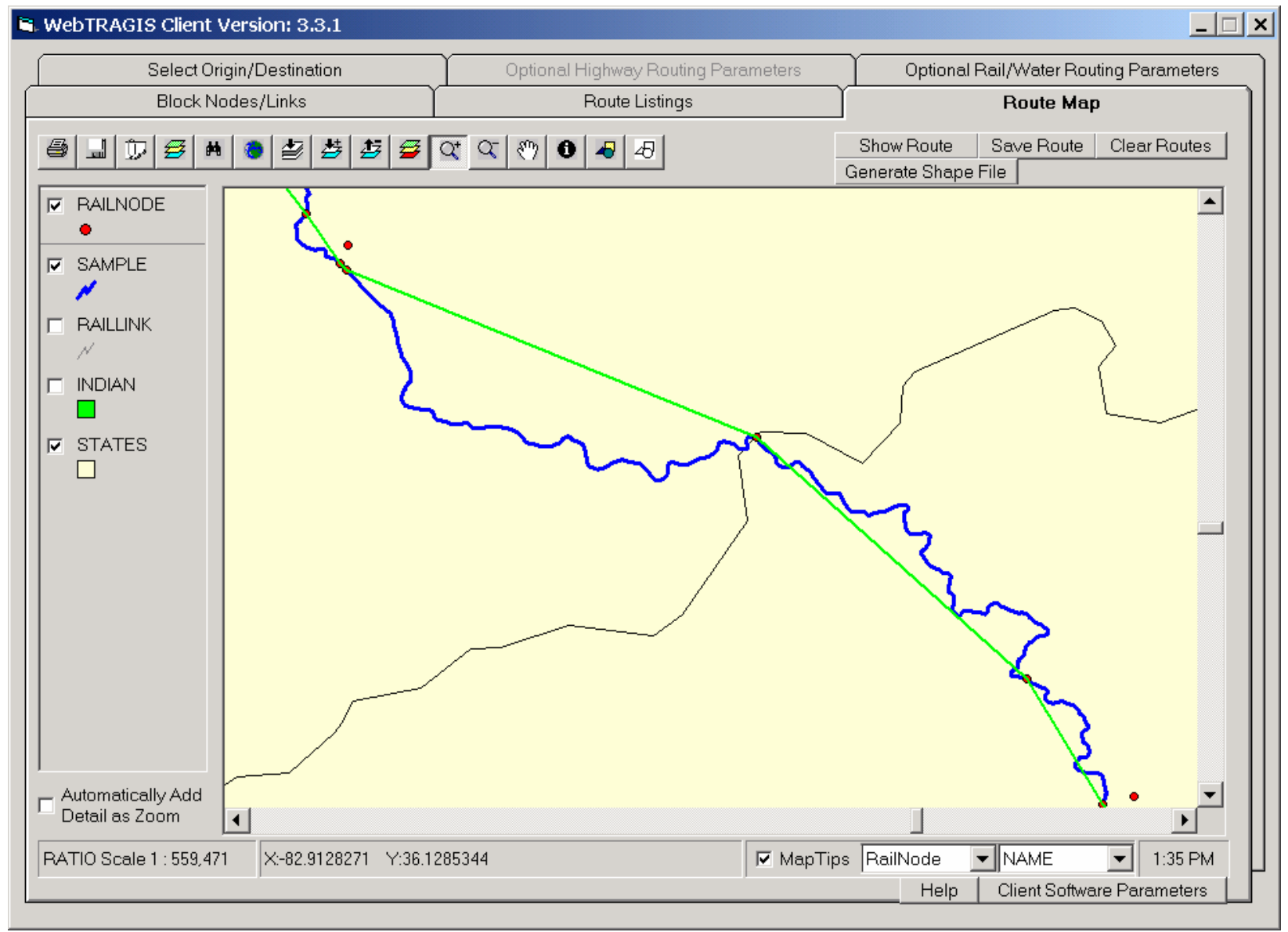

Fig. 4.22. Difference between the two rail route representations.

Previously saved routes or other shape files can be added to the WebTRAGIS map display. Further information on this capability and other mapping features of WebTRAGIS is discussed in Sect. 6 of the user's manual. 
Page intentionally left blank 


\section{WATERWAY ROUTING}

Waterway routing is the third transportation mode available in TRAGIS. The waterway database consists of both inland waterways and deep-water routes. Inland waterways include all navigable channels with a minimum depth of $6 \mathrm{ft}$. The deep-water network consists of connections between ports on the Pacific Ocean, Gulf of Mexico, Atlantic Ocean, and Great Lakes regions. Transfers between the inland and deepwater routes are treated as break-of-bulk points.

\subsection{TRAGIS WATERWAY NETWORK}

The waterway network was developed in the mid-1980s for the INTERLINE model. This waterway network was incorporated into TRAGIS when the new model was developed. Future plans are to incorporate a 1:100,000-scale waterway network into TRAGIS so all of the routing networks are based on identically scaled databases. The two waterway systems in the network are the shallow draft inland waterways, designated "IW," and the deep water commercial merchant marine system, designated "CMM."

Shapes for inland waterways are developed from 1:2,000,000-scale U.S. Geodata developed by the U.S. Geological Survey. Straight-line segments represent deep-water routes in the Great Lakes, Gulf of Mexico, Atlantic Ocean, and Pacific Ocean. Link distances are based on data from a National Oceanic and Atmospheric Administration report on distances between ports (DOC 2002). Due to the fact that many navigable rivers are state boundaries, the current waterway network links are not assigned to be within a state. As a result, population data for waterway routes are not reported by state.

\subsection{WATERWAY NODE NAMING CONVENTIONS}

The TRAGIS waterway network has a straightforward node naming conventions. Because the waterway network extends beyond the continental U.S., there may be several unfamiliar "state" abbreviations found. Nodes located in Canada have a state abbreviation of CN and nodes in the vicinity of the Panama Canal use a state abbreviation of CZ. Nodes that are located offshore are assigned to the nearest state.

Node names generally fall into five categories: port facilities, lock and dam locations, waterway junctions, facility locations, and state boundaries. Port facilities have the name of the city, followed by a semi-colon and the term "Port of" or a shortened version, depending upon the length of the city name. For example in Virginia the port of Norfolk is called Norfolk; Port of and the port of Newport News is called Newport News; Port. Lock and dam locations use the abbreviation L/D along with either the name of the location or the number of the facility. Examples of these types of nodes in Illinois include L/D 52 (Ohio), which is Lock and Dam Number 52 on the Ohio River, and Starved Rock L/D. Waterway junction node names usually include the names of the two bodies of water that meet at the node location. Examples of these node names include Miss/Illinois Jct at the junction of the Illinois and Mississippi River upstream from Saint Louis and Potomac/Chesapeake at the mouth of the Potomac River in Chesapeake Bay. Facility locations are also included in the waterway network. Nuclear power plants with barge facilities (either active or needing refurbishment) are included in the network. Also some naval facilities are included in the network. Examples of these nodes include Calvert Cliffs NP D in Maryland and Norfolk Naval Base in Virginia. The final type of node names is state boundary locations. An example of such a location is AR/MO Border, which is along the Tennessee border on the Mississippi River at the Arkansas and Missouri state line. 


\subsection{SELECT ORIGIN/DESTINATION SCREEN TAB}

To set WebTRAGIS to calculate water routes, the user needs to select the Water option in the Mode section on the Select Origin/Destination screen tab. A brief delay will occur while the appropriate data files are loaded into the model. Figure 5.1 shows the initial display of WebTRAGIS in the water model.

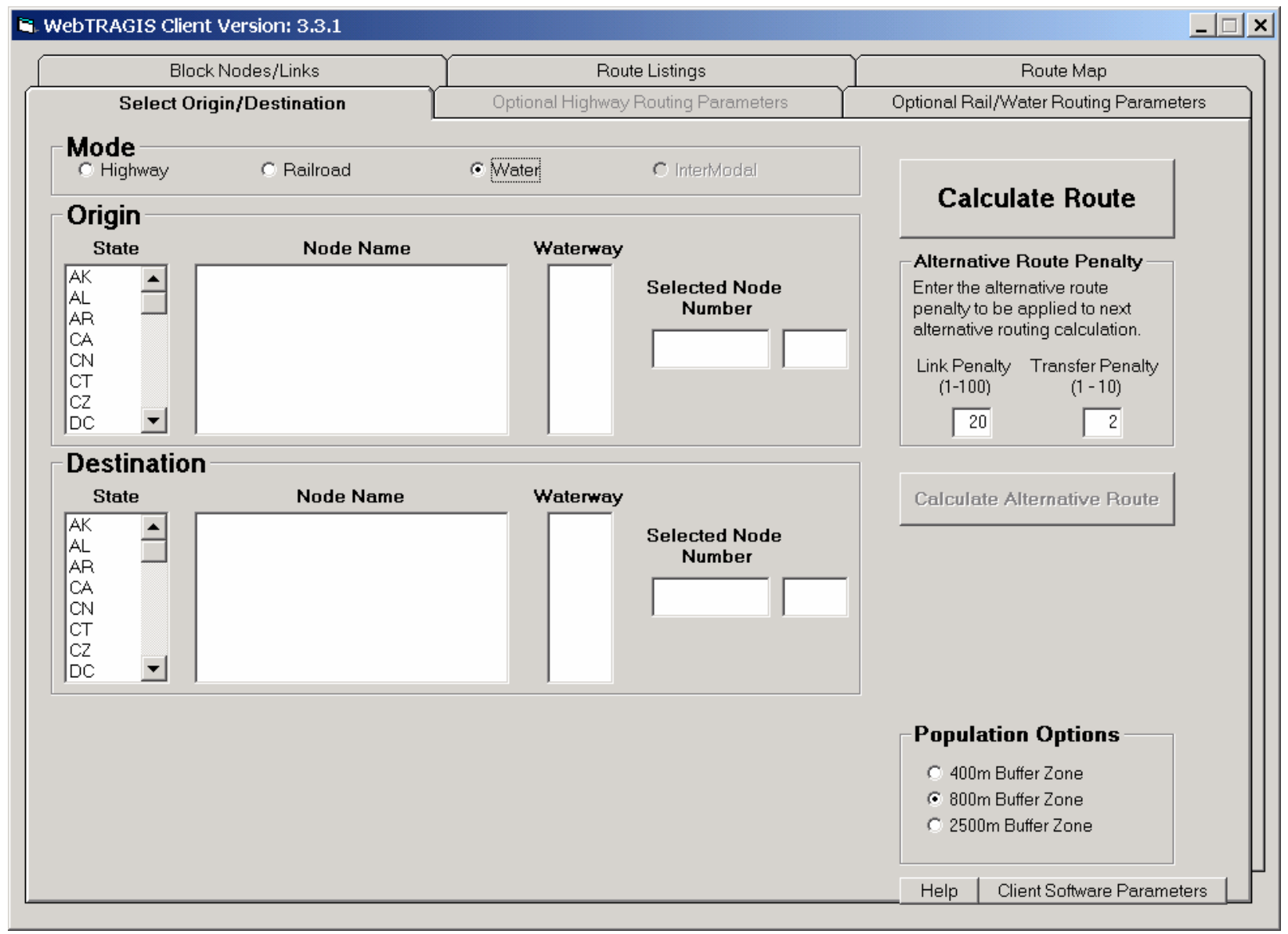

Fig. 5.1. Select Origin/Destination tab screen for water routing.

\subsubsection{Selecting an Origin and Destination}

All of the nodes in the waterway network are named, so any node can be selected as an origin or destination from the scroll lists on the Select Origin/Destination screen. The order for selecting an origin and destination does not matter. First scroll through the State list and highlight the desired state by clicking on its abbreviation. Upon selection of a state, the list of node names within that state appears in the Node Name box. Scroll through the list and select the desired node name. After choosing a node name, the node number of this waterway node is displayed in the respective Selected Node Number box. If more than one waterway system is displayed in the Waterway column, select the appropriate system. When a node is located on only one waterway system, the model automatically selects that waterway system for routing. Once both origin and destination are selected, the user may either press the Calculate Route button or select other parameters to modify the route. 
Figure 5.2 shows an example of the selection of an origin and destination. In this example, the Origin box has the State of TN (Tennessee) highlighted, the Node Name list is scrolled to the top of the list, and the Port of Chattanooga is selected. Chattanooga is only on the inland waterway system and the model automatically selects this subnetwork. The destination selected in this example is the Port of Paducah, Kentucky, which is also only served by the inland waterway system.

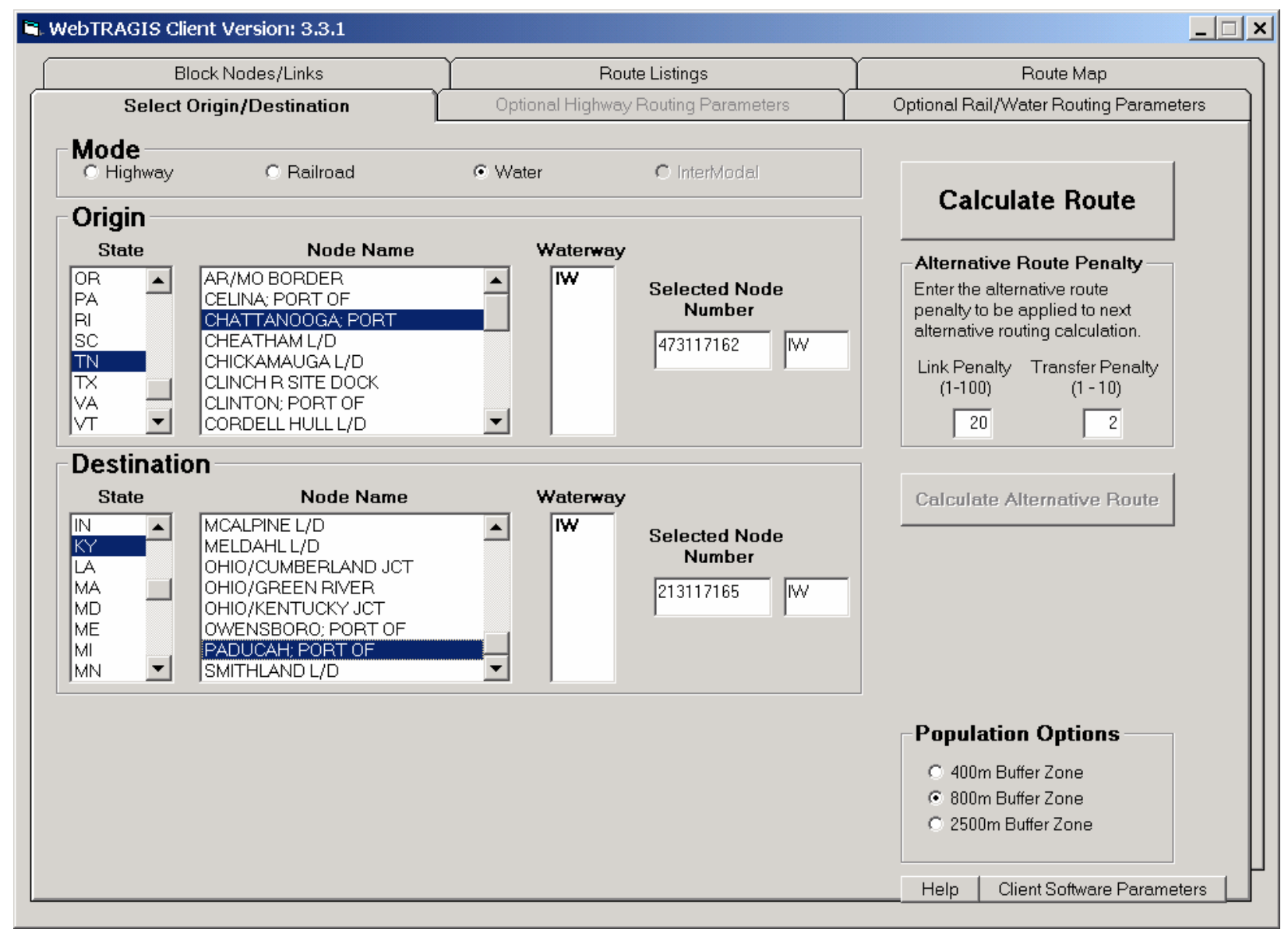

Fig. 5.2. Selection of a water origin and destination.

\subsubsection{Population Options}

In addition to route selection, one of the most important applications of TRAGIS is to provide population density and count data along routes. The population data in TRAGIS are derived from the LandScan USA 15-arc second (approximately 360 by 460 meter) grid cell population database (Bhaduri 2002). This database represents nighttime population distribution and is developed from a combination of data sources including 2000 U.S. Census Bureau block group population, roads from the Census TIGER data, slope from the NIMA Digital Terrain Elevation Data, and land cover from the USGS National Land Cover Database. Population density and count information are based on geographic buffers constructed around the transportation route. Data on three different buffer distances are available in TRAGIS - 400, 800 , and 2500 meters either side of the route. By default, the model selects the 800 meter-buffer width. To examine the population within one of the other corridor distances, select the appropriate distance within the Population Options box in the lower right hand portion of the Select Origin/Destination screen. Please note that the selection of a different buffer distance will not be executed until the Calculate 
Route button is pressed. The population along a route is displayed using the Population Data Listing button on the Route Listings screen.

\subsubsection{Calculate Route}

After an origin, destination, and population option are selected, click the Calculate Route button on the right side of the screen. If a required item has not been selected, an error message window will appear with a reminder that all origin and destination data need to be provided before a route can be calculated. WebTRAGIS will calculate the water route and then display the Route Listings window showing the standard listing for the route.

\subsubsection{Calculate Alternative Route}

After a water route has been calculated, the Calculate Alternative Route button becomes active, allowing the user to generate alternative routes. This feature is useful to determine if additional routes exist that are similar, but different than the optimal route selected by WebTRAGIS. In preparation for running an alternative route, all links comprising the current route are penalized by the Alternative Route Penalty factors. There are two separate alternative route penalty factors for water routing - link and transfer penalties. The default value for the Link Penalty is 20 and 2 for the Transfer Penalty. The Link Penalty adds an additional 20 percent to the existing impedance of each previously used link. The Transfer Penalty multiplies used interchange penalties between waterway systems by a factor of 2 . Due to the increased impedance values, alternative routes will tend to use a different set of links and interchange locations. When running multiple alternative routes, the penalization of multiple previously used links and transfers are multiplicative. Entering a new value in the Link Penalty and Transfer Penalty areas of the Alternative Route Penalty box, located between the Calculate Route and Calculate Alternative Route buttons, can change these factors. Care should be taken in adjusting these factors. To calculate an alternative route, go back to the Select Origin/Destination tab and then click the Calculate Alternative Route button. Due to the sparse nature of the waterway network, the likelihood of viable alternative routes is rare. The alternative routing feature is included in the water portion of TRAGIS for consistency between all the transportation modes in the model.

\subsection{ROUTE LISTINGS SCREEN}

The Route Listings screen tab provides access to the text-based output from WebTRAGIS. When the model generates a route, the program displays the Route Listings screen with the standard listing of the calculated routes, as shown in Fig. 5.3. A number of buttons are located along the upper edge of this screen. The function of each of these buttons is provided below.

\subsubsection{Print}

The Print button sends the information listed in the Route Listings screen to the printer connected to the PC running the client version of WebTRAGIS. The default windows printer and settings are used to print data displayed on the screen.

\subsubsection{Save As}

The Save As button provides a method of writing the information listed in the Route Listings screen to a standard text file. The function of this button is the same for whatever previous button is used to display data on this screen, be it the Detailed Listing, Population Data Listing, Route Info, or Standard Listing button. When this button is pressed a Save As window is opened, which allows the user to select 
the appropriate drive, directory, and file name where the text information from the screen will be saved. By default, this function saves all files with the ".txt" extension.

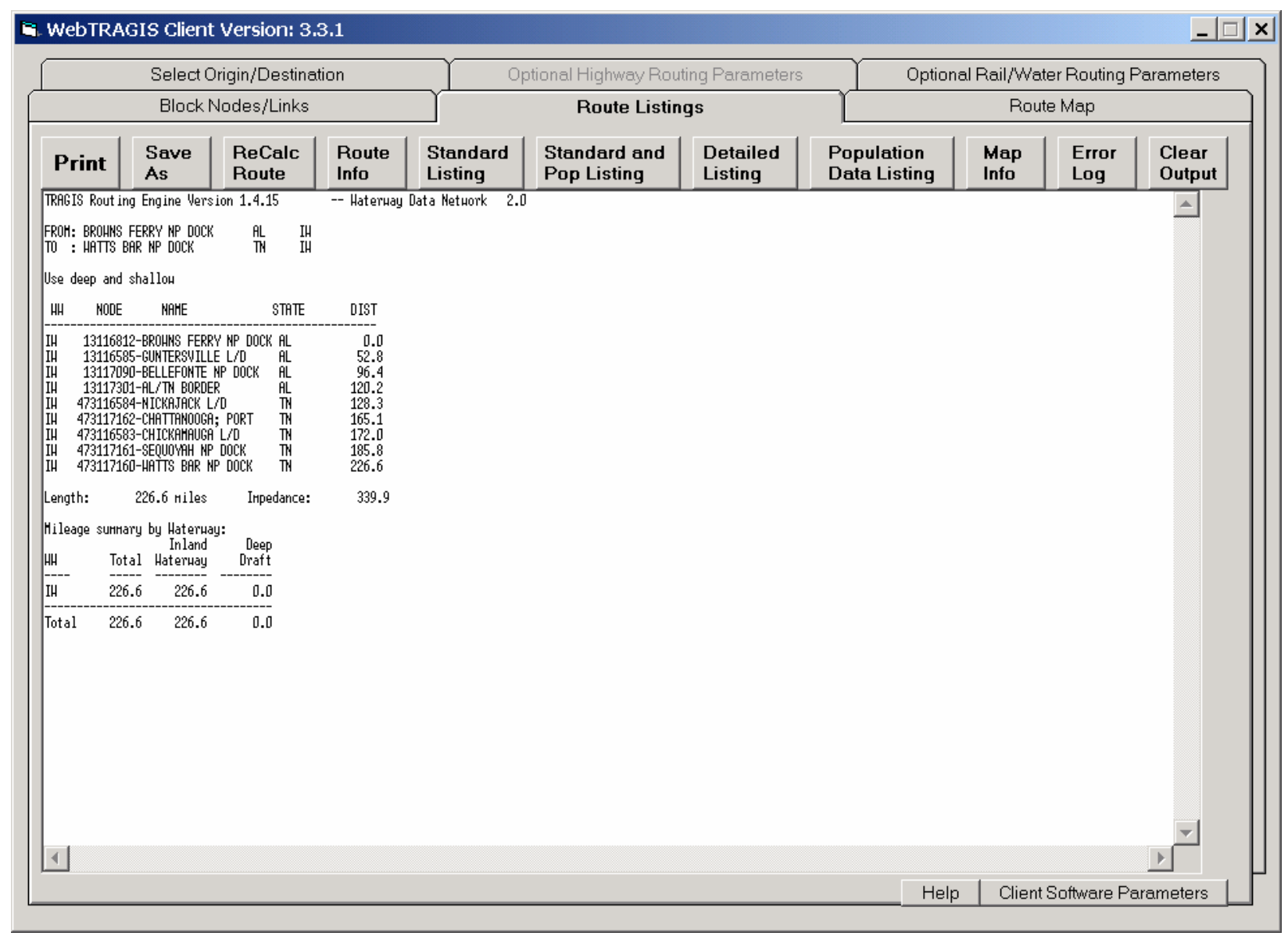

Fig. 5.3. Standard routing listing for a water route.

\subsubsection{ReCalc Route}

The ReCalc Route button opens a window to select a previously saved Route Info file. After this file is selected, WebTRAGIS will recalculate the route based on the parameters in the Route Info file. The normal user will rarely, if ever, need to use this feature. This feature is primarily used to assist users who are having problems with the model and allows TRAGIS development staff to be able to reproduce user problems.

\subsubsection{Route Info}

The Route Info button displays the most recent set of parameters used to create a route (such as the origin, destination, option settings, blocked nodes and links, etc.). This set of parameters is sent over the Internet to the TRAGIS server to calculate the route. If the user experiences any problems with generating a particular route, this button should be used to examine the parameter settings. If further assistance is required, the Route Info data should be saved in a file, using the Save As button, and then the saved file can be e-mailed to the TRAGIS development staff for debugging. 


\subsubsection{Standard Listing}

The Standard Listing button displays the route description of the most recent route calculated. (The program displays this listing following the calculation of each route.) Figure 5.3 provides an example of the standard listing of a route between the Browns Ferry Nuclear Power Plant dock in Alabama and the Watts Bar Nuclear Power Plant Dock in Tennessee.

For barge routes, the standard listing identifies the origin and destination at the top of the display, all the nodes along the route, any transfer locations between the shallow and deep water networks, the total length and impedance of the route, and mileage summary for the route. The main portion of the route listing identifies the waterway system, node number, node name, state, and cumulative distance for each node along the route.

\subsubsection{Standard and Pop Listing}

The Standard and Pop Listing button displays a combination of the information shown with both the Standard Listing (see Sect. 5.4.5) and the Population Data Listing (see Sect. 5.4.8) buttons.

\subsubsection{Detailed Listing}

The Detailed Listing button provides additional information on the route. Every node on the route is listed along with information on link characteristics. Link information includes the link number; the distance of the link; and the impedance value for the link. As with the Standard Listing, summary information by railroad and state follows the route listing. Figure 5.4 provides and example of the detailed listing for a route between the Browns Ferry Nuclear Power Plant dock in Alabama and the Watts Bar Nuclear Power Plant Dock in Tennessee.

\subsubsection{Population Data Listing}

The population density and county information is viewed by clicking the Population Data button. A listing of the population density information for the route is displayed. Figure 5.5 shows the population data listing for a route between the Browns Ferry NP Dock and the Watts Bar NP Dock. The basic table includes 11 population-density categories ranging from 0 to over 9996 people per square mile. The entries in the table show the distance that the route travels in each category. At the end of the table, summary information is provided for the route. This information combines the data from the 11 categories into rural, suburban, and urban groups and also provides a weighted population-density value for each of these groups. This information is used as route specific input for risk analysis using the RADTRAN (Neuhauser 2000) or RISKIND (Yuan 1995) computer codes. Waterway links have not been coded to specific states because a number of waterways coincide with state boundaries. The total population count within the selected buffer zone is also provided at the end of the listing. For the example shown in Fig. 5.4, 15,264 people reside within 800 meters of either side of the route.

Input data for the RADTRAN model requests population density and distance data for rural, suburban, and urban areas. The basis for these three categories is:

- Rural Categories below 139 people per square mile,

- Suburban Categories between 139 and 3326 people per square mile,

- Urban Categories above 3326 people per square mile.

The model reports the route specific weighted population density and distance data (in both English and metric units) for the three population categories. 


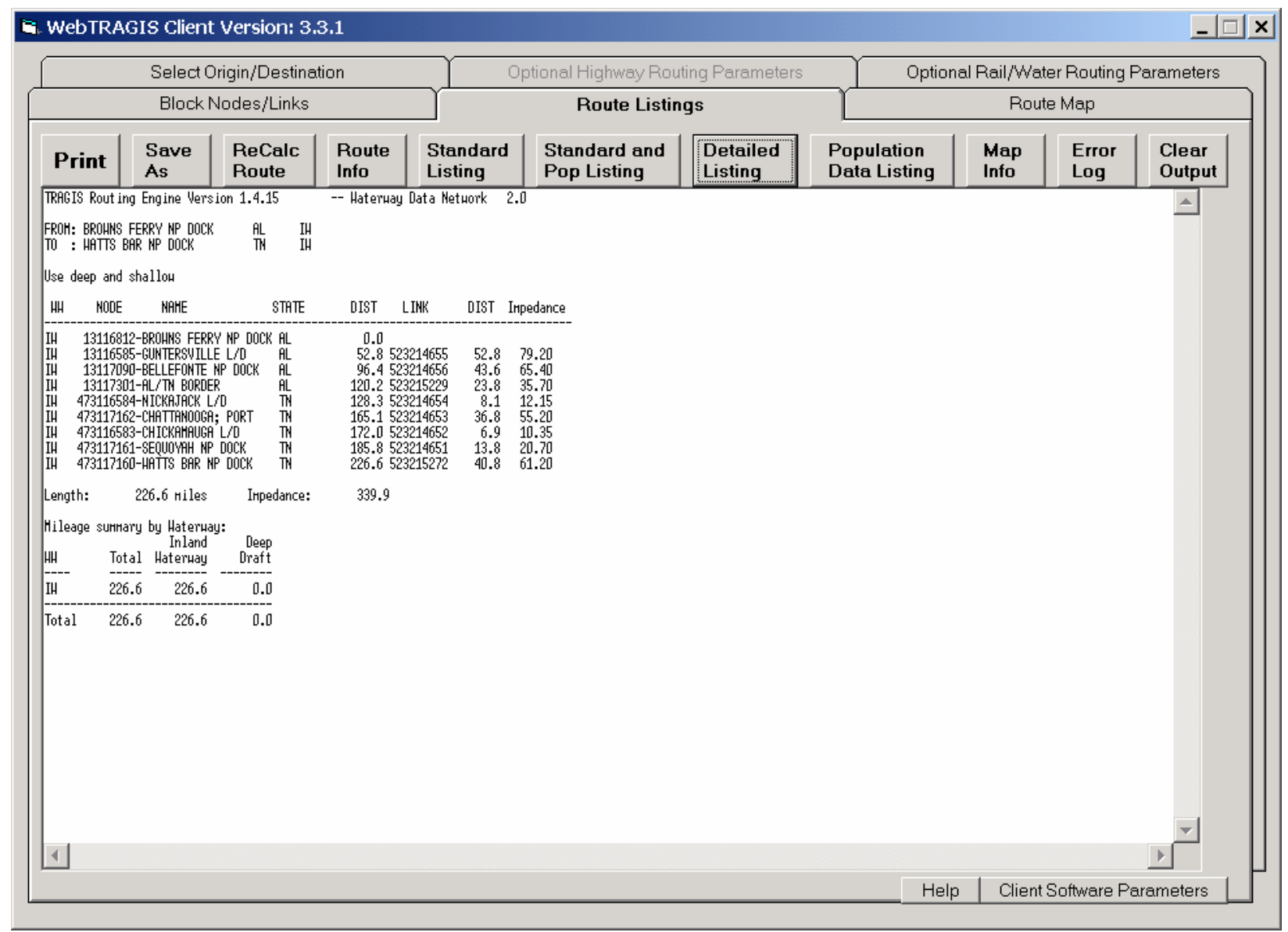

Fig. 5.4. Detailed barge route listing.

With the improved calculation methods used to determine the population numbers, the user should note that these data are only estimates. Also, these numbers represent nighttime population figures and that daytime population distributions are significantly different. It is best to use these population figures for global comparisons of different routes rather than an absolute value.

\subsubsection{Map Info}

The Map Info button displays a text file with the end point coordinates of each individual link for the most recently calculated route. This data only provides node coordinates and does not provide the shape coordinates for the links. Information on this screen is used to draw the straight-line segments on the Route Map screen discussed in Sect. 5.7.3. Most users will not have a need to view this data.

\subsubsection{Error Log}

If the model generates an error, the error will automatically be displayed in the Route Listings screen.

The Error Log button is used to redisplay any error message that the model generates on the server. If the error message does not provide sufficient assistance to remedy the problem, please contact one of the TRAGIS development staff regarding the error message. Telephone numbers for the TRAGIS development staff can be determined by clicking on the Help button on the lower edge of any WebTRAGIS screen. 


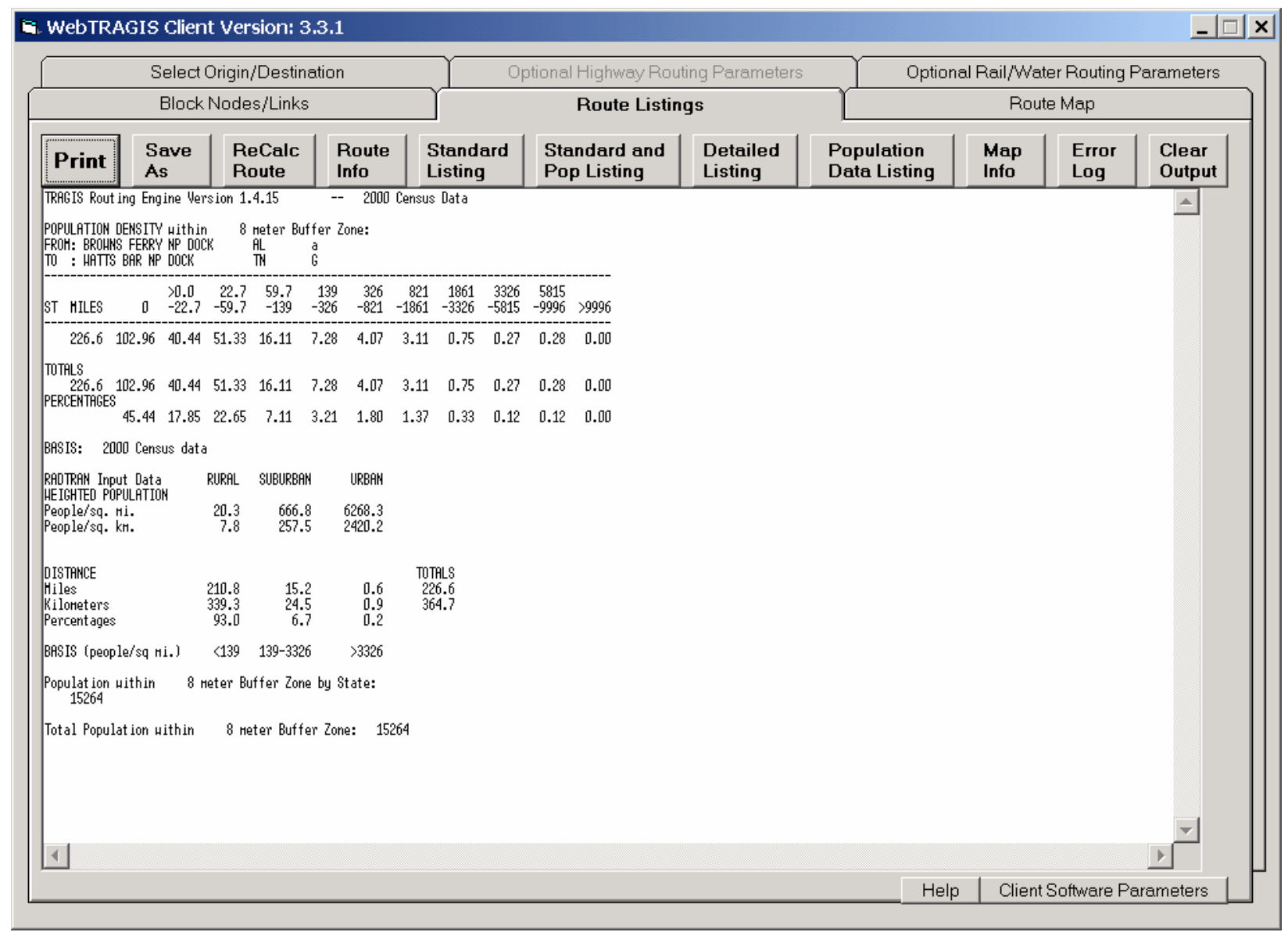

Fig. 5.5. Example of the population data listing for a water route.

\subsubsection{Clear Output}

The Clear Output button will remove the contents of all the WebTRAGIS output files (the route info, standard listing, detailed listing, population data listing, map info, and error log files) that can be viewed in the Route Listings screen. The output files for WebTRAGIS are downloaded from the server to the client computer following the calculation of each route and overwrite earlier copies. This button provides a means to delete the output files on the client computer.

\subsection{OPTIONAL RAIL/WATER ROUTING PARAMETERS SCREEN}

The Optional Rail/Water Routing Parameters screen provides access to several parameters that control the waterway routing functions. When water or rail is the active mode, the Optional Rail/Water Routing Parameters tab is available, and the Optional Highway Routing Parameters tab is greyed out, indicating that this tab cannot be selected. The Optional Rail/Water Routing Parameters screen only lists the water option parameters when WebTRAGIS is in water mode.

The selection of the Optional Rail/Water Routing Parameters tab will display a window similar to that shown in Fig. 5.6. This window identifies the additional options that can be altered. The following sections discuss the various water options. 


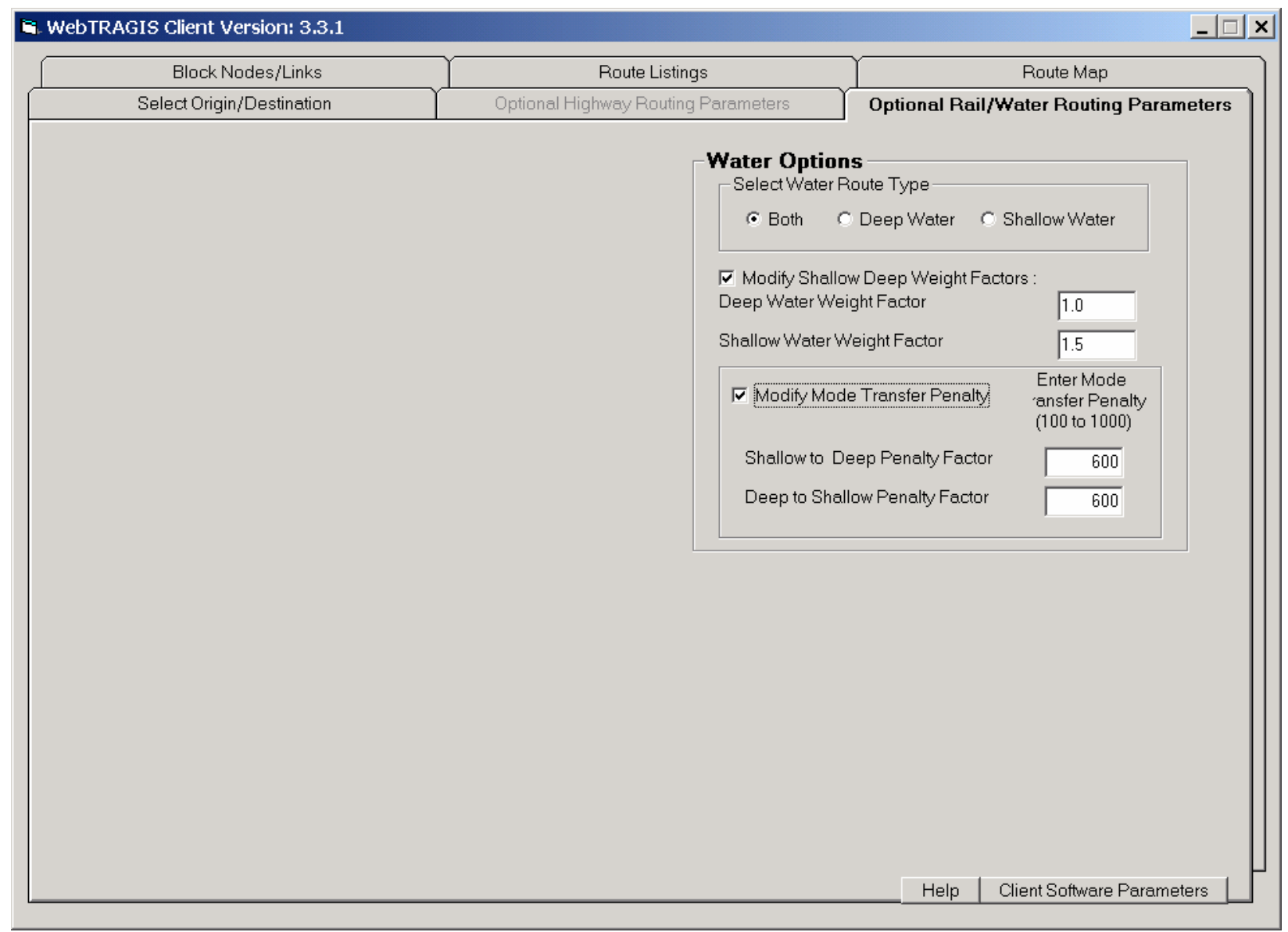

Fig. 5.6. Optional water routing parameters.

\subsubsection{Water Route Type}

The TRAGIS waterway routing network consists of two interconnected subnetworks. One network is the shallow draft inland waterway system. This network has a limitation of a 6-foot draft. The other waterway system is the deep-water network, which is primarily located along the Atlantic, Gulf of Mexico, and Pacific coast as well as the Great Lakes and St. Lawrence River.

WebTRAGIS, by default, allows the use of both the deep and shallow water networks. With the Select Water Route Type box, the user is allowed to select only one of the water networks. As shown in Fig. 5.6, Both is selected (both deep and shallow water networks). By clicking on either Deep Water or Shallow Water, the model will only use that single network to calculate water routes.

\subsubsection{Shallow and Deep Water Weight Factors}

The TRAGIS model calculates waterway routes by minimizing the total impedance between the origin and destination. The impedance is defined as a function of distance, type of waterway system used, and any transfers between waterway systems. The impedance equation is:

$$
L=\operatorname{Min}\left\{\sum_{i}\left(f d_{i}\right)+\sum_{n} T_{n}\right\}
$$

where 
$\mathrm{L}=$ total impedance of a route;

$\mathrm{f}_{\mathrm{i}}=$ weighting factor for link $\mathrm{i}$, with

$\mathrm{f}_{\mathrm{i}}=1.0$ for deep water links, and

$\mathrm{f}_{\mathrm{i}}=1.5$ for shallow water links;

$\mathrm{d}_{\mathrm{i}}=$ distance for link $\mathrm{i}$, in miles;

$\mathrm{T}_{\mathrm{n}}=$ transfer penalty factor at node $\mathrm{n}$.

By checking the Modify Shallow Deep Weight Factors box on the Optional Rail/Water Routing

Parameters screen, the user can alter the penalty factors used for the two waterway networks in Eq. (5). Figure 5.6 shows the Modify Shallow Deep Weight Factors checked. Two entry boxes are available, one labeled Deep Water Weight Factor and the other Shallow Water Weight Factor. Experienced users can enter new values to alter the weighting factors for calculating waterway routes.

\subsubsection{Mode Transfer Penalty}

TRAGIS places a 600-unit penalty on transfers between the deep and shallow water networks as shown in Eq. (5). The Modify Mode Transfer Penalty provides a method to alter the transfer penalty between the two waterway networks. (Note: This procedure is for experienced users only.) Figure 5.6 shows the Modify Mode Transfer Penalty checked, which displays two boxes that are used to alter the transfer penalty between the two waterway systems. The new value entered in the Shallow to Deep Penalty Factor changes the value of all transfers between shallow to deep waterway systems. Entering a new value in the Deep to Shallow Penalty Factor changes the penalty factor between deep to shallow waterway systems.

\subsection{BLOCK NODES/LINKS SCREEN}

The Block Nodes/Links option is recommended only for experienced users. This option allows the user to temporarily block portions of the waterway network database by blocking nodes and links. These features can be useful when there is a need to analyze various scenarios such as determining a route that avoids a damaged lock. The Block Nodes/Links screen is divided into two boxes, one to block nodes and the other to block links. An example of the Block Nodes/Links window is shown in Fig. 5.7. Each of the two functions is discussed in the following sections.

\subsubsection{Node Blocking}

Nodes can be blocked in the waterway database thus making all the links that emanate from the node ineligible for routing. If a blocked node is on the only possible path between an origin and destination, the TRAGIS routing engine will be unable to calculate a route. If such a condition occurs, the Route Listings screen will display "ERROR: No path found. Exit Code: 21."

To block a water node, go to the Block Nodes section on the Block Nodes/Links screen and scroll through the State list and click on the postal abbreviation of the desired state. Next the Node Name list will display all node names for that state. Click on the node name to temporarily block that node. A final step is required if this node is served by both waterway systems. Select the Waterway to be blocked. After the waterway system is selected, the model will automatically add the selected node and waterway system to the Blocked Node Numbers list. Entering the node number and waterway system in the Manual Node Entry box can also be used to block a node.

Figure 5.7 shows an example of blocking the Port of Baton Rouge node. This node is on the both the deep water (CMM) and inland waterway (IW) and systems. Only the deep-water portion of this node has been 
blocked, simulating the loss of ability for deep-water ships from accessing this port. Barge traffic can still pass through Baton Rouge with this scenario.

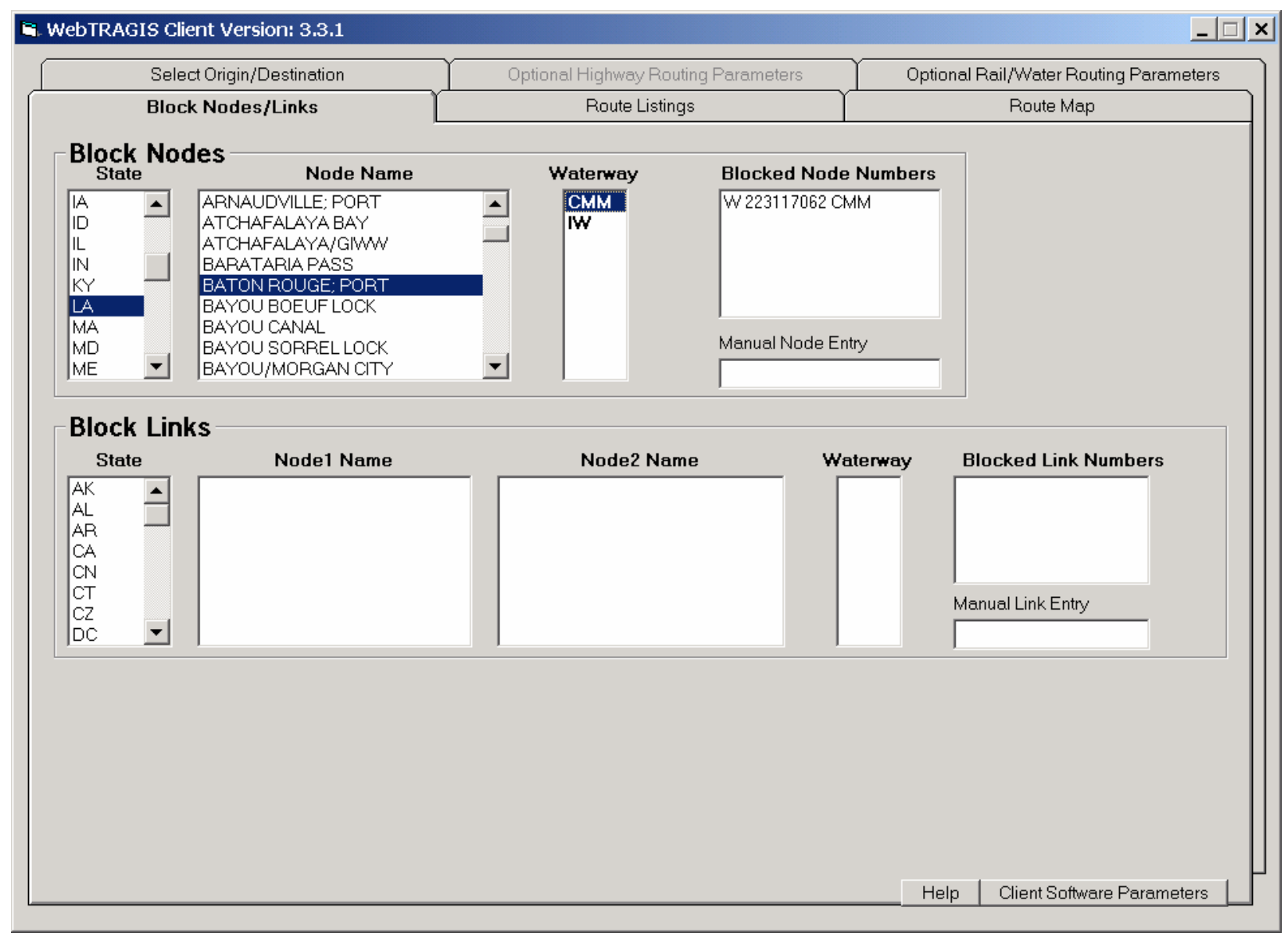

Fig. 5.7. Water node blocking.

A node number can be removed from the Blocked Node Numbers list by double-clicking on the entry. The Blocked Node Numbers list does not provide the name of blocked nodes, but the route listing does provide both the node number and name of blocked nodes.

\subsubsection{Link Blocking}

Links in the barge network can be modified to temporarily remove them from consideration on a route. As with node blocking, if the blocked link lies on the only possible route between the origin and destination, the model will generate an error message stating that no path could be found. The method of blocking a link involves identifying the link by the two end node names. The first step is to go to the Block Links section of the Block Nodes/Links screen and select a state by scrolling through the State list and clicking on the postal abbreviation of the desired state. Following the state selection, the Node1 Name list is populated with all the node names in the state. Select the desired node by clicking on the name. After this selection, the Node2 Name list will be populated will all of the nodes connected to the selected node from the Node1 Name list. Select the appropriate location from the Node2 Name list by highlighting the name. Finally, the Waterway list is populated with the waterway systems available on this link. If only one waterway system is shown, the link number and waterway system will be displayed 
in the Blocked Link Numbers list. But if both waterway systems are available on the link the final step is to select which system should be blocked on the link. Clicking on the appropriate waterway abbreviation results in its blocking. Entering the link number and waterway system in the Manual Link Entry box can also be used to block a link.

For each waterway system selected, two entries appear in the Blocked Link Numbers list. Each entry lists the same link number and waterway system, but the first entry ends with a ' 1 ' and the second with a ' -1 '. Links are represented in the model as one-way connections. The ' 1 ' link extends from the from-node to the to-node. Conversely, the '-1' link extends the other direction, from the to-node to the from-node. The best way to determine the direction of a link is to block the link and then calculate a route. The route listing identifies all blocked links and shows the to and from names for the ' 1 ' and ' -1 ' directions of the link. To block a link for only one direction, double click on the opposite direction entry in the Blocked Link Numbers list and it will be removed from the list.

Figure 5.8 shows an example of waterway link blocking. In this example, the state of Mississippi is selected and Lock B on the Tennessee-Tombigbee Waterway is selected as the Node1 Name. This node connects to two other nodes identified in the Node2 Name list. These two nodes are Lock A and Lock C. Lock $\mathrm{C}$ is selected and both directions of link number 523215013 are shown in the Blocked Link Numbers window list.

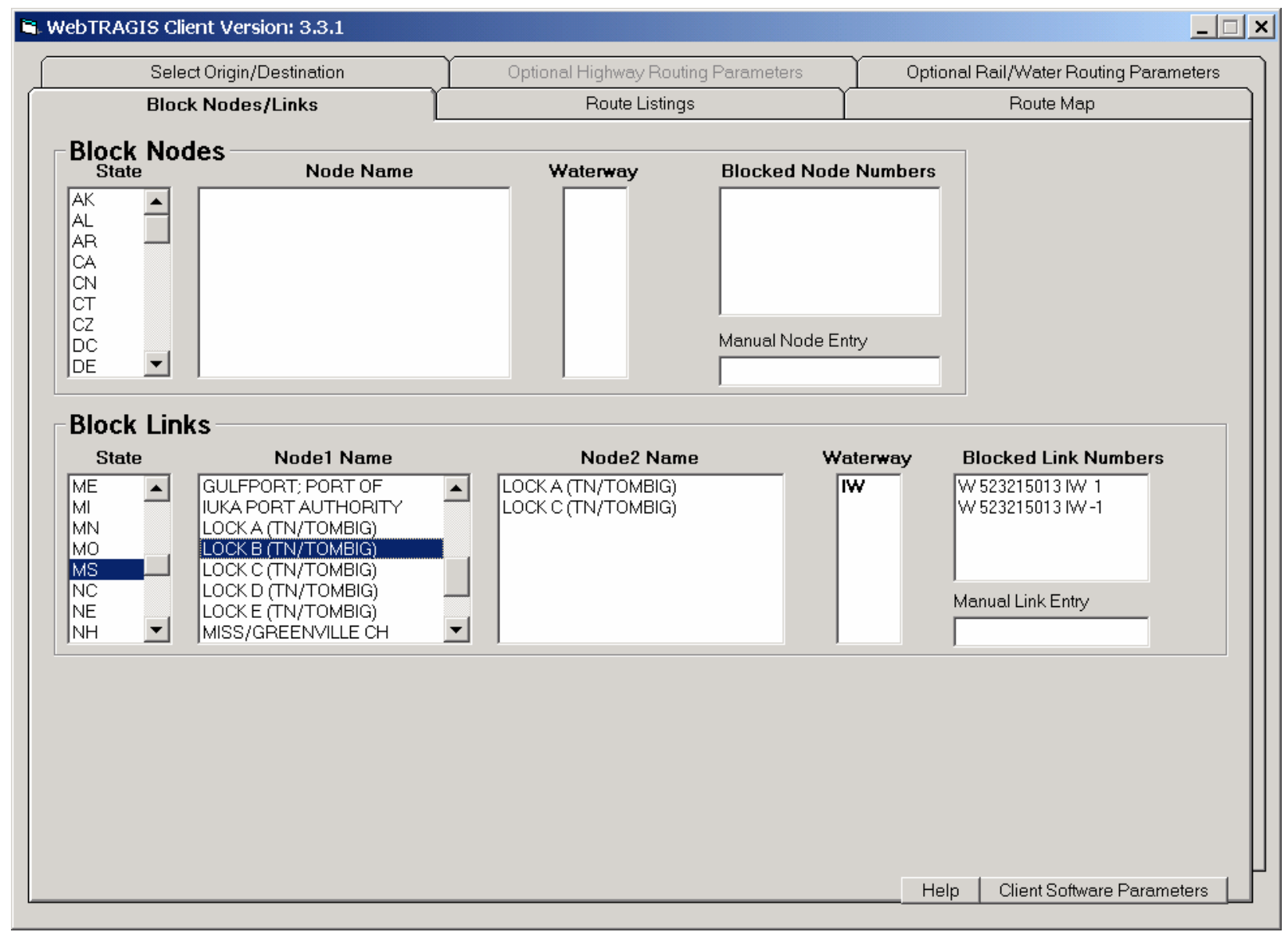

Fig. 5.8. Water link blocking. 


\subsection{ROUTE MAP SCREEN}

Figure 5.9 shows the Route Map screen for the water mode of WebTRAGIS. This section describes the functionality specific to the rail portion of the model. Section 6 of this user's manual provides a detailed description of common features to the highway, rail, and waterway Route Map screens.

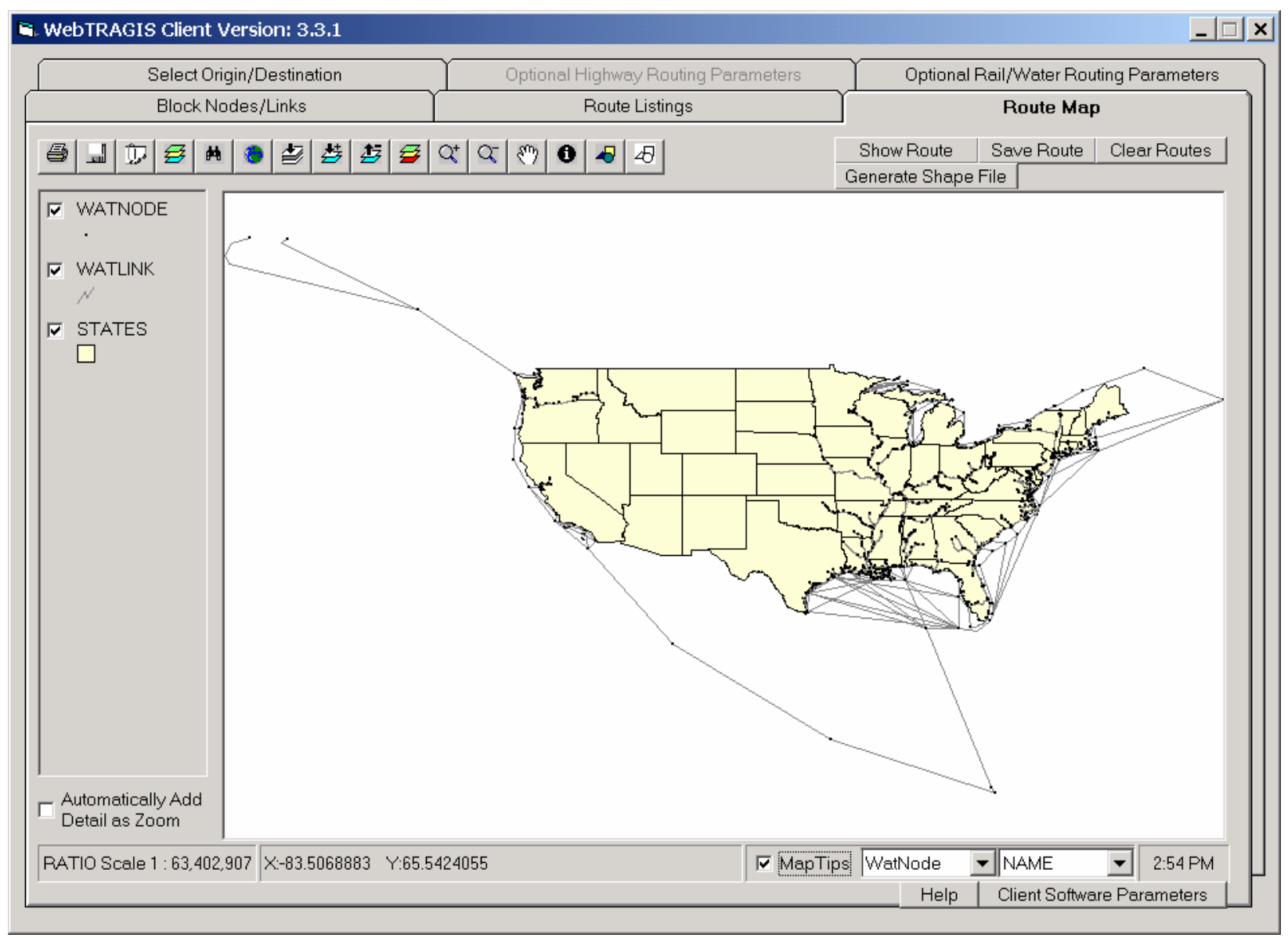

Fig. 5.9. Water route map screen.

\subsubsection{Water Coverages}

The Route Map screen consists of the primary window displaying a map of the continental U.S. and a legend area on the left hand portion of the screen. Three coverages are shown in the legend-Watnode, Watlink, and States. The Watnode coverage consists of points representing each node in the waterway network database. The waterway network is shown as one coverage, called Watlink. The other coverage is State that shows the state boundaries. All three coverages are initially displayed on the screen. By clicking on the check box, the check mark can be removed, which caused the display to be redrawn without that coverage shown on the map. 


\subsubsection{Using MapTips}

In the lower right hand center of the screen, MapTips has been checked, as shown in Fig. 5.9. (The MapTips item is checked for this example to assist the discussion of this feature. By default, MapTips is not checked when the Route Map screen is displayed.) Two pull down boxes appear to the right of the MapTips label. The left pull down box is used to select the coverage, in this case the WatNode coverage. The right pull down box is used to select an individual attribute of coverage. When the WatNode coverage is selected, the attribute field defaults to Name. Zooming into a specific area best uses the MapTips feature. Move the pointing device over a node and the name of the node will be shown on the screen. MapTips can be used to examine the attributes for any of the coverages shown on the Route Map screen.

\subsubsection{Displaying Routes}

WebTRAGIS provides two methods of displaying a calculated route in the Route Map screen. The simplest method draws straight-line segments between nodes. This technique is very fast and is sufficient for first draft national scale maps. The top row of buttons on the upper right hand portion of the Route Map screen is used to control the drawing of routes with this method. The Show Route button will display the most recently calculated route. (Even if you have just started WebTRAGIS, the most recently calculated route means the last route you have created with the model.) If you are creating a series of routes, the Show Route button can be pressed after the calculation of each to display multiple routes on the screen. Each successive route is displayed in a different color on the screen. The Save Route button saves all the routes displayed on the screen in one file in the ESRI shape file format. When this button is pressed, a save as window is opened to allow the user to select the folder where the file is stored. The Clear Routes button will clear all routes displayed on the screen.

The Generate Shape File button provides the capability to make a map of the route showing the full detail of the geographic shape available with the 1:2,000,000-scale waterway network available in WebTRAGIS. This process is slightly more complicated compared to the method described in the previous paragraph, but is the preferred method if large scale (showing small areas) maps are needed. When the Generate Shape File button is pressed a Save As window is opened, this allows the appropriate location and file name to be selected. The file is saved in the ESRI shape file format, which can be used with ESRI GIS software, such as ArcView and ArcGIS.

Previously saved routes or other shape files can be added to the WebTRAGIS map display. Further information on this capability and other mapping features of WebTRAGIS is discussed in Sect. 6 of the user's manual. 


\section{ROUTE MAPS}

The mapping capability in WebTRAGIS is one of the primary features of the model. The mapping capabilities of WebTRAGIS are written with ESRI MapObjects software. Some users may be familiar with ESRI products and be able to use the mapping features with little additional assistance. Other users who are not familiar with GIS software will find this section of the user's manual especially helpful to learn more about GIS mapping techniques and tools.

Figure 6.1 shows the basic display of the Route Map screen. This section of the user's manual will discuss the use of the variety of features available in TRAGIS in the following order. The button bar is located along the upper left portion of the screen. The upper right portion of the screen has a set of four buttons that control display of routes. The map legend area is located along the left side of the screen. Finally, the MapTips feature, coordinate information and ratio scale are located along the bottom edge of the screen.

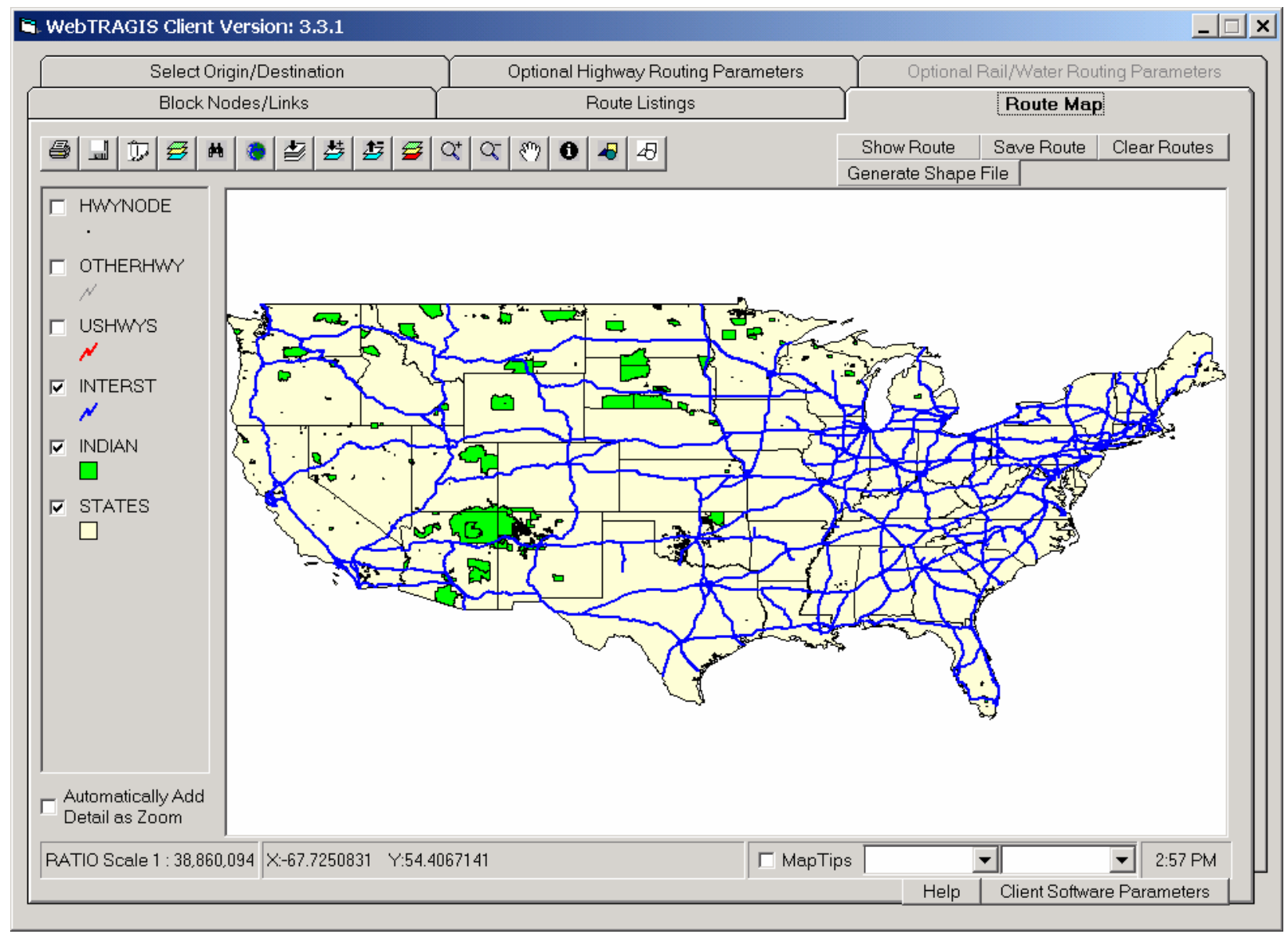

Fig. 6.1. Route map example. 


\subsection{BUTTON BAR}

Along the upper left portion of the Route Maps screen are a series of sixteen buttons. This is called the button bar. These buttons provides a set of useful functions for working with WebTRAGIS maps. Each button has an icon indicating the function of that button. When the pointing device of the PC is placed over a button a small popup box will appear with a short description of that button's function. The following sections describe the function each button, starting with the print button on the left of the button bar.

\subsubsection{Print}

The leftmost item on the button bar is the Print button. This button can be identified by the printer icon. When this button is pressed, a Print the Map window, as shown in Fig. 6.2, is displayed. This window controls the printing of the current map display.

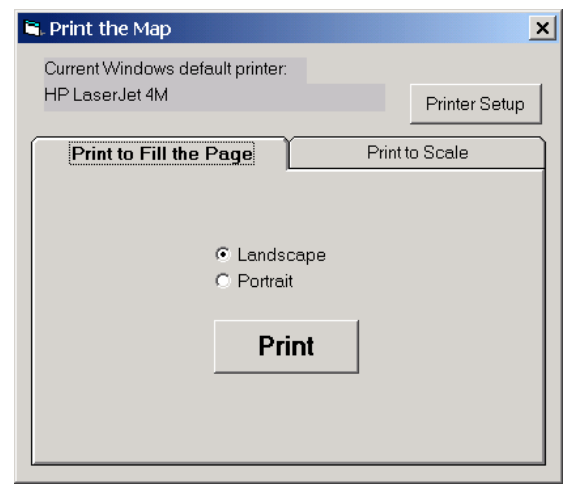

Fig. 6.2. Print the map screen with the print to fill the page tab active.

The upper left Print the Map window, the current default printer identified. By pressing the Printer Setup button, the user can select the other printers that are available. When this window is displayed, the Print to Fill the Page tab is active. This tab screen allows the user to print the map as either landscape or portrait. Pressing the Print button sends the information shown in the Route Map screen to the printer. Figure 6.3 shows the Print the Map window with the Print to Scale tab selected. With this option the user can select the ratio scale for the map. When this feature is selected, the map is printed in portrait mode.

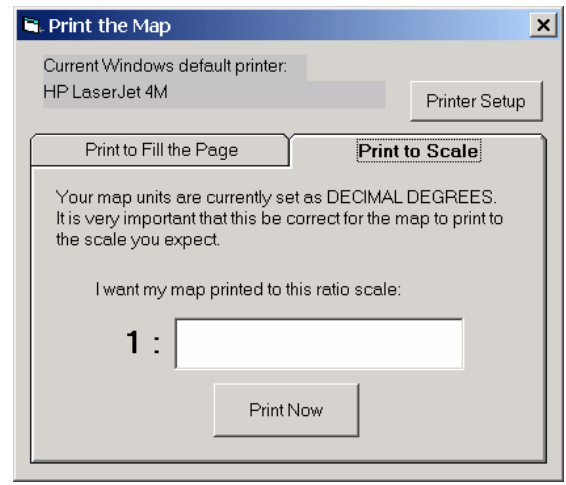

Fig. 6.3. Print the map screen with the print to scale tab active. 


\subsubsection{Save Displayed Map to Graphics File}

The second button on the button bar is the Save Displayed Map to Graphic File button. It can be identified by the disk icon. Figure 6.4 shows the window displayed when this button is pressed. This button allows the user to save the current map display as an enhanced windows metafile (.emf type file) or as a bitmap file (.bmp type file), as indicated in the lower portion of the Save As window. The default is as an enhanced metafile because a metafile can be resized without appreciable loss of detail. Select the drive and directory where the file will be saved in the Save in box. Next enter the filename and extension (either .emf or .bmp) in the File name box. Finally, press the Save button. The Save Displayed Map to Graphic File function makes a copy of the display only within the map area of the Route Map screen.

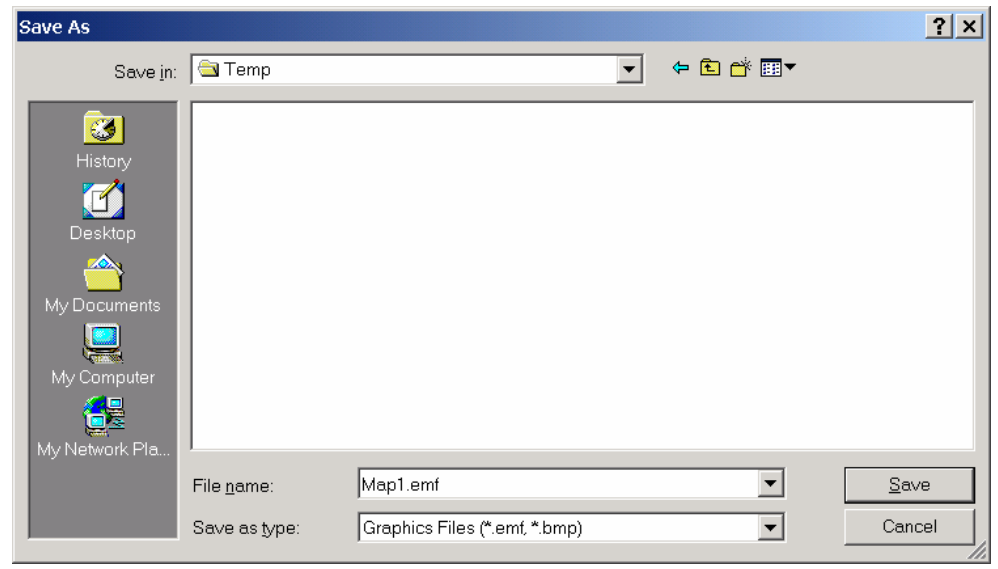

Fig. 6.4. Save as window.

\subsubsection{Copy}

The Copy button is the third button from the left and is symbolized by a paper clip on a piece of paper. Pressing this button automatically copies the current displayed map onto the Windows clipboard, so it can be pasted into other Windows applications. As with the Save Displayed Map to Graphic File button, only the map display is copied. Neither of these buttons copy the legend or the entire WebTRAGIS window. (If a copy of the entire WebTRAGIS window is desired for insertion into a document, such as in this user's manual, hold down the Alt key and press the Print Screen key on your keyboard to capture the active screen on the computer display to the clipboard. Then go to your Windows application and paste from the clipboard.)

\subsubsection{Map Properties}

Three-colored layers symbolize the Map Properties button, which is the fourth button from the left on the button bar. This button opens a window shown in Fig. 6.5 that allows the user to change properties of the map. The Add Layer button in the upper left of the Map Properties window is the same button as discussed in Sect. 6.1.8. Below the Add Layer button is an empty white box with the text "click to select map background canvas color." The default background color for the map is white, as shown in this display. By clicking in this area, a color selection window, as shown in Fig. 6.6, is displayed. To select a different background color, click on the desired color box and press the OK button. The Map Properties window allows the user to control whether scrollbars appear on the map and if the border area of the display window has a three dimensional appearance (both of which are selected). On the right hand side of the Map Properties window are three different options with pull down menus. It is suggested that the default values be used. Press the Close button finish actions with the map properties. 


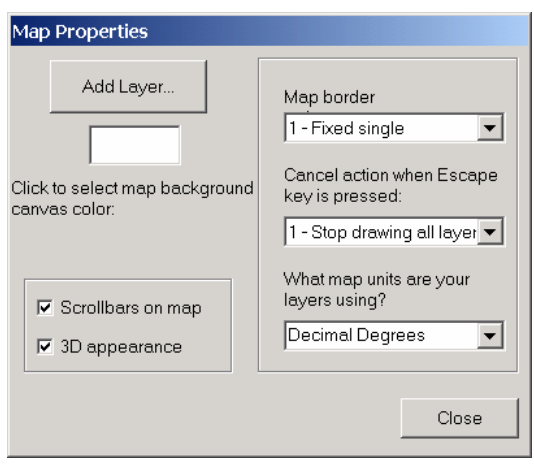

Fig. 6.5. Map properties window.

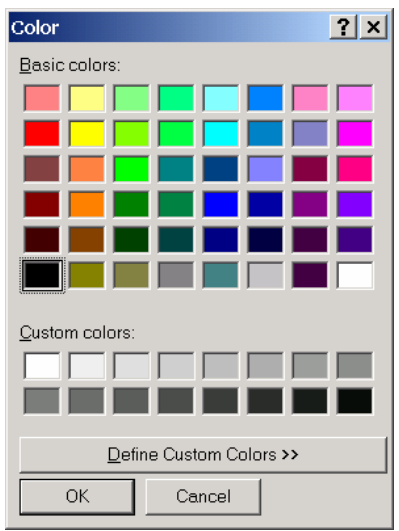

Fig. 6.6. Color selection window.

\subsubsection{Search}

The Search Map Information Files button is symbolized by a binocular. The map layers have data elements associated with them, such as highway sign, speed limit, node names, etc. This button opens a window that allows the user to search these data elements. It is suggested that users not use this function because it not easy to operate.

\subsubsection{Display Full Extent of Map}

The Display Full Extent of Map button is presented by a globe icon. If the map has been zoomed into a smaller area, pressing this button changes the display such that the entire map is displayed with the viewing area. Figure 6.1 shows the map display at the full extent.

\subsubsection{Display Map to Extent of Active Layer}

The Display Map to Extent of Active Layer button is symbolized by an arrow pointing into three layers. The legend area displays the individual map coverages or layers. For example, Fig. 6.1, shows the initial view for the highway portion of WebTRAGIS. Six different map layers are shown in the legend with three displayed, indicated by the checkmarks. None of the layers are active in Fig. 6.1. To activate a layer, click on the title of the layer. Figure 6.7 shows that the Interst layer in the legend is active because it has a three dimensional effect of appearing raised, compared to the other layers. The function of the Display 
Map to Extent of Active Layer button is to zoom in on the active layer so that this layer extends to the edge of the map display. It can be noted by comparing Figs. 6.1 and 6.7 that the map has been zoomed so that I-5 in Oregon is at the western edge of the map and I-95 in Maine is at the eastern edge of the map.

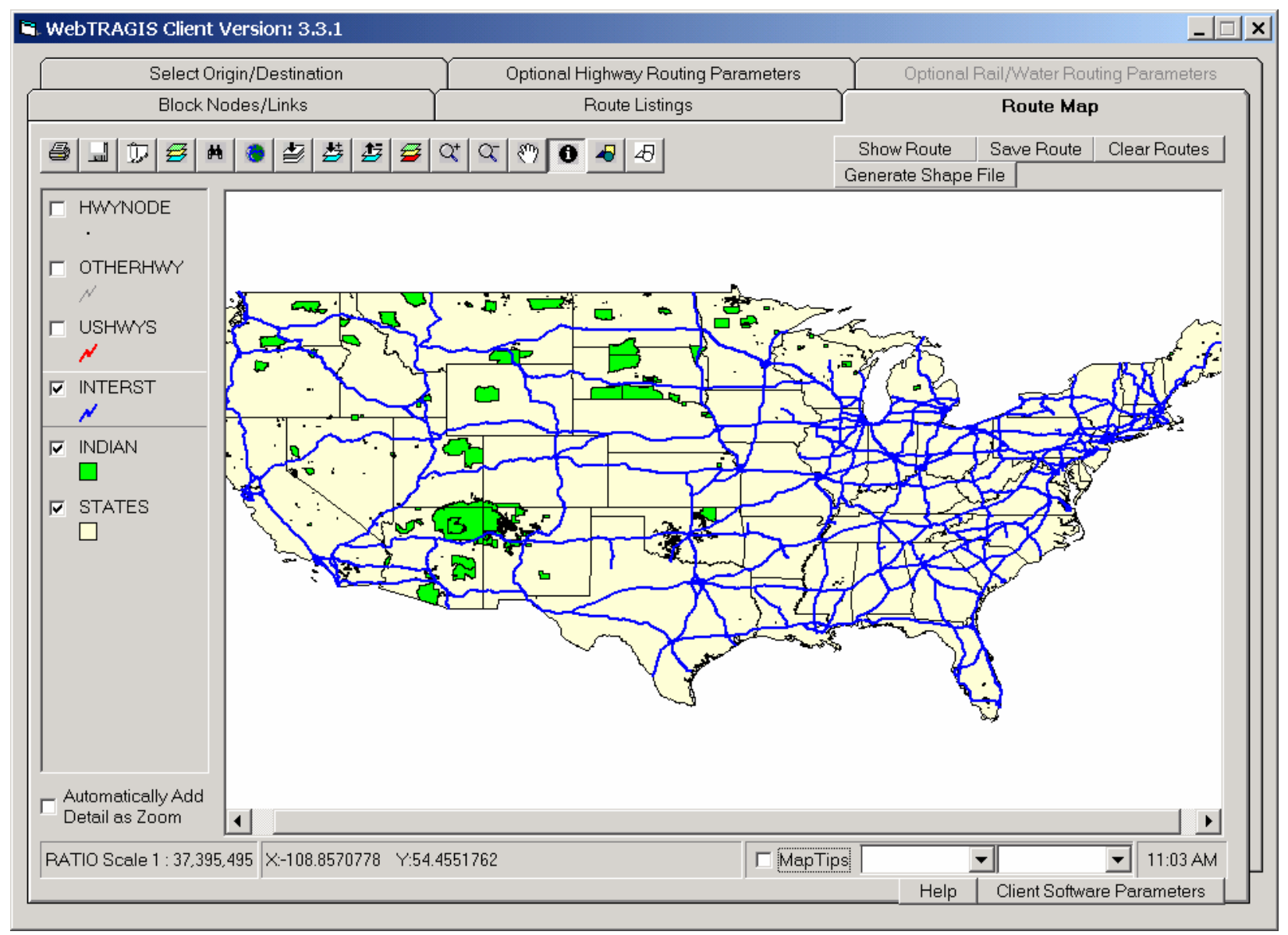

Fig. 6.7. Map displayed to extent of active layer.

\subsubsection{Add Map Layer}

The Add Map Layer button is represented with an icon showing an arrow pointing down into two colored layers with a plus sign to the right of the arrow. This button is used to add additional layers to the map display or to redisplay a route that was previously saved. When this button is select, a Select file for new layer dialog window is displayed, as shown in Fig. 6.8. This window allows the user to browse through the file systems on their computer to select an ESRI shapefiles (.shp type files) to display. After selection of a shapefile, press the Open button and this file will be added to the Route Map window.

\subsubsection{Remove Active Map Layer}

The Remove Active Map Layer button is represented with an icon showing an arrow pointing up from two colored layers with a minus sign to the right of the arrow. Pressing this button will remove the active layer from the map. For example, in Fig. 6.7, the Interst layer is the active layer. If the Remove Active Map Layer is pressed, the Interst layer will be removed from the map and the legend. An error message will be displayed if this button is pressed and no layer is currently active. To make a layer active, click on the coverage name in the legend box 


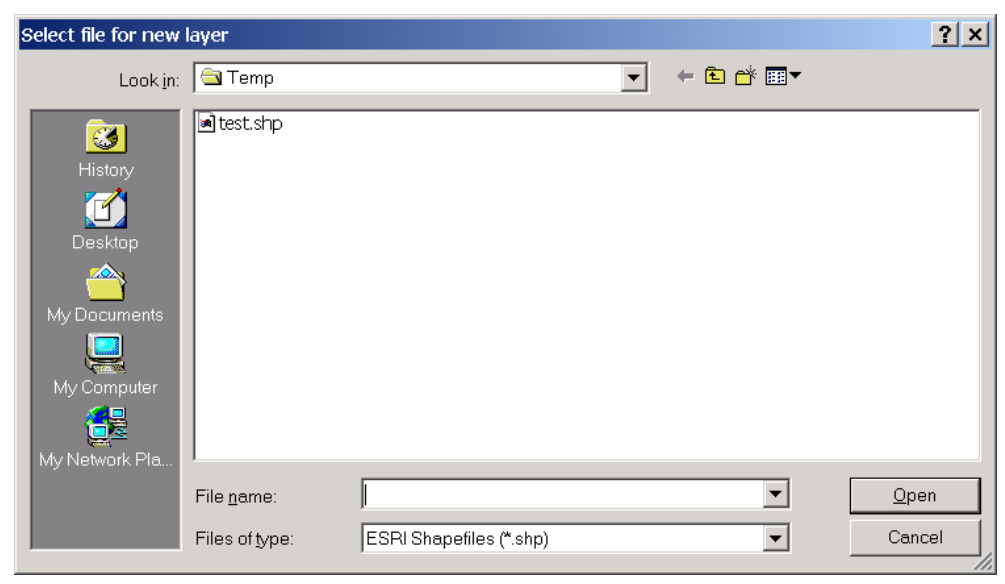

Fig. 6.8. Select file for new layer window.

\subsubsection{Modify Map Layer Properties}

The Modify Map Layer Properties button has an icon with three colored layers (light green, yellow, and red). This button opens a Symbol properties for the $\mathbf{x x x}$ layer window, where "xxx" represents the name of the active layer. This window is shown in Fig. 6.9 and allows the user to modify the properties of the active map layer. The active layer is denoted in the legend with the three dimensional raised appearance. A layer can be made active type by clicking on its name in the legend.

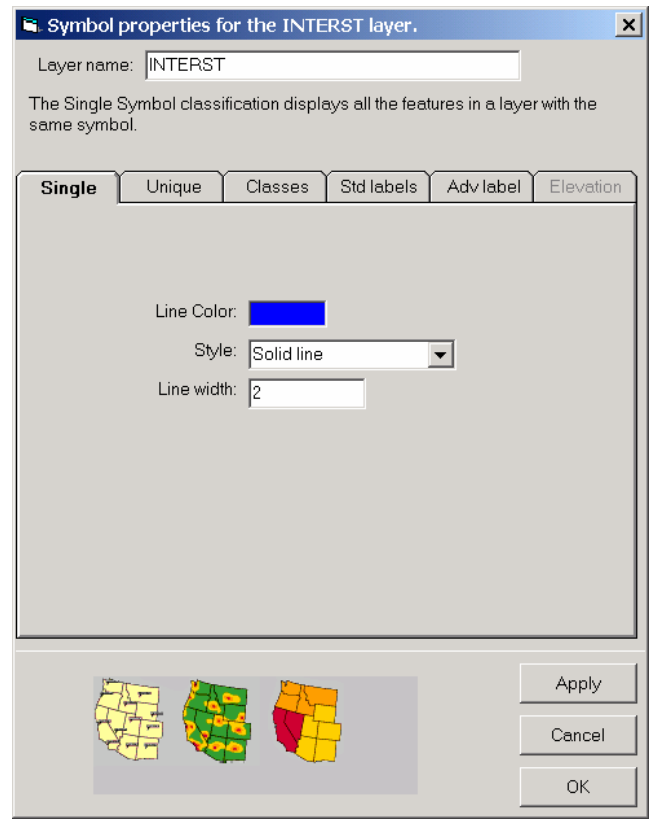

Fig. 6.9. Single tab of modify map layer properties window.

The modify map layer properties window contains six tabs that can be used to change the appearance of the map layer, depending upon the feature in the active layer. The tab names are single, unique, classes, standard labels, advanced labels, and elevation. If any of the tabs are not applicable to the selected layer, that tab will appear in a grey tone, as is the elevation tab in Fig. 6.9. A text description explaining the 
function of the currently selected tab is provided below the name of the layer being modified, which is identified in the box at the top of the window. One drawback of some of these features is the limited ability to control color and line width on attribute classifications. Each tab is described in the following paragraphs, primarily for layers with linear data. The format varies slightly for point and polygon layers.

The Single tab allows the user to display of all the features in a layer with the same symbolization. Figure 6.9 shows the Symbol Properties window for the Interst layer. All roads in the Interst layer are displayed with a blue solid line with a line width of two. To change the color of the roads in this layer, click the box identified as line color and a color window, shown in Fig. 6.6, is displayed. To select a different color, click on the desired color in the color window and then click the OK button. To change the style of the line, click on the pull down arrow and select from the list of option. Finally, to change the width of the line, enter a new value, between 1 and 5, in the line width box. Changes to the map display will not occur until the Apply button is pressed.

The Unique tab allows for a classification of one of the attribute fields of the selected layer. With this tab, a field in the layer database can be mapped with each value mapped as a unique symbol. The Field pull down menu is used to select the available field for mapping. First select a field and then press the Reset legend button. The classification will then appear in the left portion of the window. Repetitive clicking of the Reset legend button results in different legend color combinations. Changes to the map display will not occur until the Apply button is pressed. In the example shown in Fig. 6.10, the Urban_Flg is selected. This attribute only has two values ( 0 or 1$)$. If the selected field has too many unique values, an error message will be displayed.

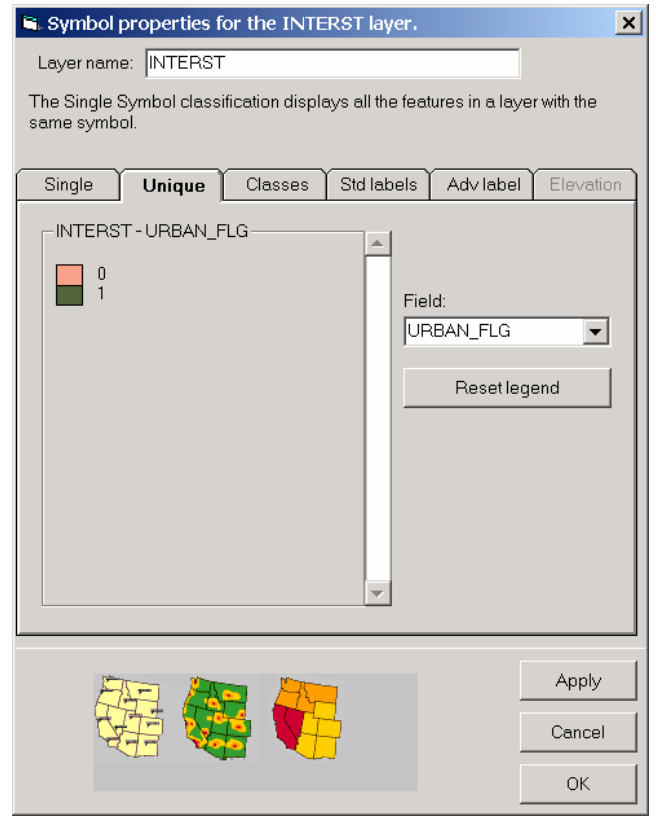

Fig. 6.10. Unique tab of the modify map layer properties window.

The Classes tab allows for a classification of one of the numeric attribute fields of the selected layer. With this tab, a numeric field in the layer database can be mapped into a selected number of classes. The Numeric Field pull down menu is used to select the available field for mapping. First select a field from the list and then the number of classes that the data will be mapped into. A graded color scale will be used to represent the classes. Click on the start color and the color window, identical to the window shown in Fig. 6.6, will be displayed. Select a color for the start of the color ramp and then press the OK button on 
the color window. Repeat this process for the end color and then press the Reset legend button. The classification will then appear in the left portion of the window. Changes to the map display will not occur until the Apply button is pressed. In the example shown in Fig. 6.11, the Speed field and five classes are selected. The model automatically calculates the class breaks and the color gradation.

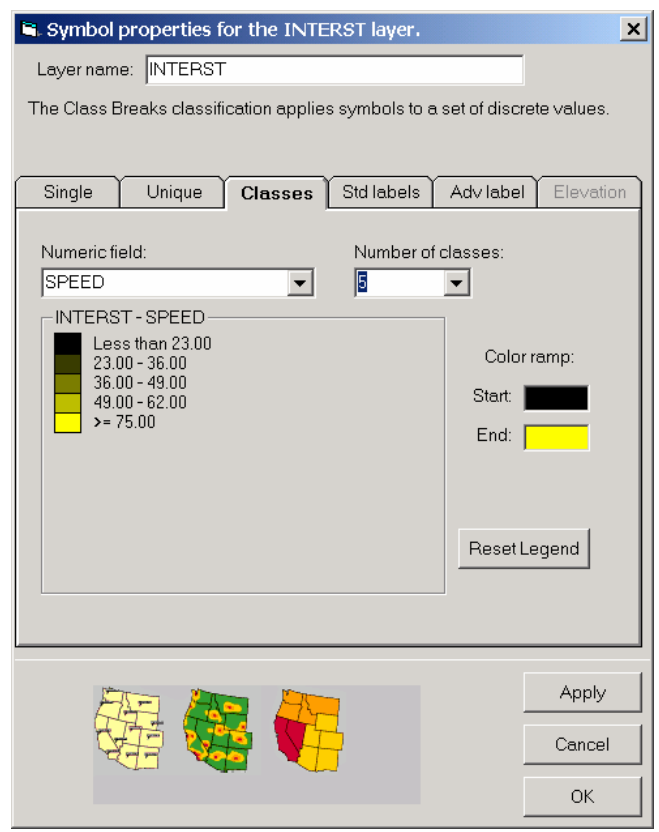

Fig. 6.11. Classes tab of the modify map layer properties window.

The Standard and Advanced Labels tabs are used to automatically label attributes from a selected field on the map display. Both of these tabs are rather specialized and somewhat difficult to use. The Elevation tab is only available for layers with elevation data.

\subsubsection{Zoom In}

The Zoom In button is represented by an icon with a magnifying glass and a plus sign. This tool is used to zoom in or to enlarge an area within the display. To perform a zoom in action, click the Zoom In button so that this button icon appears indented. Next move the pointing device over the display window. The pointing device now appears as the magnifying glass with a plus sign in it. Position the pointing device at a corner of the area desired to be enlarged and hold down a mouse button. While still holding the mouse button down, move the pointing device to the opposite corner of the desired area and release the mouse button. The display window will be redrawn to enlarge the selected area.

\subsubsection{Zoom Out}

The Zoom Out button is represented by an icon with a magnifying glass and a minus sign. This tool is used to show a larger geographic area in the display. To perform a zoom out action, click on the Zoom Out button so that this button appears indented. Next move the pointing device over the display window. The pointing device now appears as a magnifying glass with a minus sign in it. By clicking a mouse button, the map display is redrawn to a new scale which is twice the previous scale. For example, if map has been zoomed into a scale of 1:3,000,000 (note that the ratio scale of the map is shown in the lower left corner of the screen), one click with the zoom out button will change the scale of the map to 1:6,000,000. 
Another click on the screen will change the scale to 1:12,000,000. Continued clicking on the map with zoom out function will eventually return the map to the full extent, the same scale available by clicking the Display Full Extent of Map button.

\subsubsection{Pan}

The Pan button is symbolized with a hand icon. This function allows repositioning of the map within the display window while maintaining the scale of the map. As with other buttons, first click on the Pan button and then move the pointer over the display window. The pointer will be displayed as a hand. Hold a mouse button down and drag the display within the window.

\subsubsection{Identify}

The Identify button is represented by an icon with a lowercase letter " $\mathrm{i}$ " inside a black circle. This button is used to display attribute information for the active layer. To use this function, first click on the Identify button in the tool bar. Next, examine the legend on the left side of the Route Map window to determine the active layer. (The active layer will have a three dimension raised appearance.) To create or change the active layer, move the pointer over the layer name and click the title of the layer. Next click on a feature in the display, and an Identify Results window will appear which lists all the attribute variables and their values for that feature. Figure 6.12 shows an example of a selected link on the Interst layer. The scroll bar on the right side of the attribute box can be used to view the other attributes. To remove the Identify Results window, move the pointer over the title bar of this window and press the $\mathbf{X}$ button.

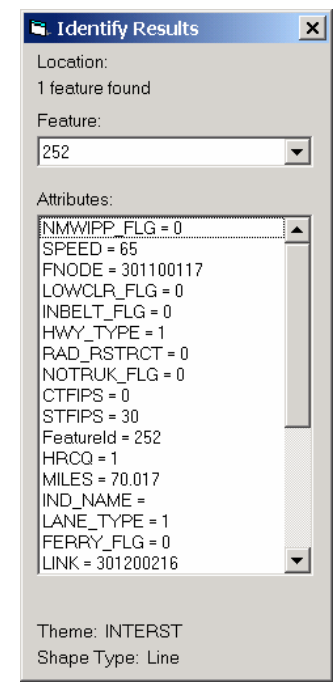

Fig. 6.12. Identify results window.

\subsubsection{Add Graphics to Map}

The Add Graphics to Map button is represented by an icon with three colored geometric shapes. This button allows the user to add text, dots, straight lines, multiple-point lines, rectangles, circles, and polygons to the map. After the Add Graphics to Map button is selected, an addition button bar appears to the right of the showing icons for the different features available. Figure 6.13 shows the additional graphics buttons. 


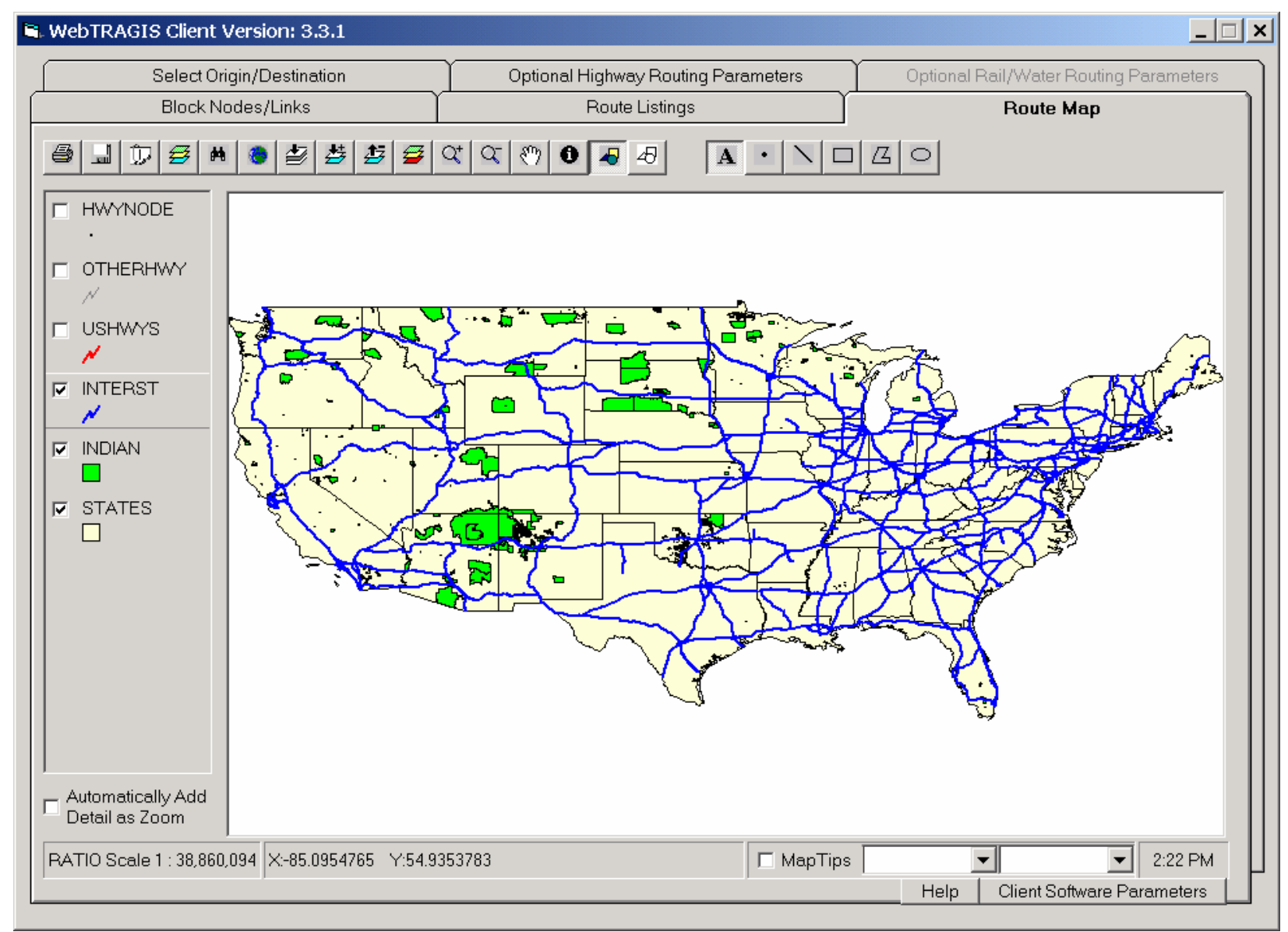

Fig. 6.13. Additional buttons with add graphics to map button activated.

The graphics button with the letter "A" icon allows text to be added map display. Click on the map where the text is to be placed. The text will be centered at the clicked point. A window will appear allowing the user to enter a string of text. Press the OK button to add this text to the map. Additional text can be added by clicking at a different point. Figures 3.15 and 4.13 provide examples of adding text graphics in WebTRAGIS.

The graphics button with a dot allows a point symbol to be added to the map display. Click on the map where the symbol is to be placed. A small red triangle will be centered at the clicked point. An additional point can be added by clicking at a different location. Figures 3.13 and 4.3 provide examples of adding point graphics in WebTRAGIS.

The graphics button with a diagonal line icon allows a line to be drawn on the map display. After this button is selected, every click on the map display creates a vertex on a multi-segmented line. Double clicking creates an endpoint for line drawing.

The graphics button with a rectangle icon creates a filled polygon with four sides. To draw a rectangle, hold down the mouse button at the location for one corner of the polygon and then continue holding the mouse button while moving to the opposite corner. When the mouse button is released, the rectangle is completed. 
The graphics button with a multi-sided shape icon creates a filled polygon. To draw a variable shaped polygon, click the mouse button at the location for each corner of the polygon and double click the mouse button at the final corner. The program will automatically close the polygon by drawing a side between the ending and starting points.

The rightmost graphics button, represented by an ellipse shaped icon, is used to draw circular shapes on the. To draw a circular shape, hold down the mouse button at the location for the center of the circle and then continue holding the mouse button while moving to the desired radius. When the mouse button is released, the circle is completed.

The flexibility of adding graphics to WebTRAGIS maps is somewhat limited. Currently, the graphics cannot be adjusted on the display after initial placement and the user does not have the ability to change symbolism, text size, or style. Also, if the scale of the map is adjusted, the size and placement of text and point graphics are not adjusted by the change. It is suggested if a large amount of graphics needs to be added to a final map that WebTRAGIS should be used to generate the route files and another more powerful GIS application, such as ArcView 3.x or ArcGIS 8.x, be used to finalize the product.

\subsubsection{Remove Added Graphics from Map}

The Remove Added Graphics from Map button is the right-most item on the button bar. It is represented by an icon with three black and white geometric shapes. Clicking this button will remove all graphics added to the map with the Add Graphics to Map button.

\subsection{DISPLAYING ROUTE BUTTONS}

Four buttons are located in the upper right portion of the WebTRAGIS Route Map screen are used to display the most recently calculated route. The top row, consisting of three buttons, is used to process quick straight-line segment of routes. The single button on the second row is used to create a shape file of the calculated route. Each of these buttons is discussed below.

\subsubsection{Show Route}

The Show Route button draws the most recent calculated route as straight-line segments between node points on the route. This is a very quick method of drawing a route and may be entirely sufficient for national level maps. The disadvantage of this method is that most geographic detail along the route is not maintained. If geographic fidelity is of paramount importance, the Generate Shape File button should be used. With the Show Route button, the model temporarily draws the route onto the map display and automatically assigns a color to the route. The only way to change the color is to press the Show Route button another time.

\subsubsection{Save Route}

The Save Route button saves any route displayed with the Show Route button on the map into an ESRI shapefile (.shp type) format. When the Save Route button is pressed, a Save As window, identical to the window shown in Fig. 6.8, is opened to allow the user to select the drive, directory, and name for the file. After the file saved this map layer is added to the top of the map legend. The Modify Map Layer

Properties button can be used to change the line color, style, and width. Be aware that is multiple routes have been displayed with the Show Route button that all of those routes would be saved into the same file with the Save Route function. 


\subsubsection{Clear Routes}

The Clear Routes button clears all routes displayed on the map with the Show Route button. This button does not remove any map layer appearing in the legend of the Route Map screen. To remove any map layer from the legend, use the Remove Active Map Layer button, discussed in Sect. 6.1.9.

\subsubsection{Generate shape file}

The Generate Shape File button provides the capability to save a route showing the full geographic detail of the routing networks available in WebTRAGIS. When this button is pressed, a Save As window, identical to the window shown in Fig. 6.8, is opened to allow the user to select the drive, directory, and name for the file. The file is saved in the ESRI shape file (.shp type) format, which can be used with other ESRI GIS software, such as ArcView 3.x and ArcGIS 8.x. Press the Save button after selecting the file name. It may take about a minute to process the shape file, depending upon the routing network and computer speed. As the file is being processed, a red message will appear over the Generate Shape File button area of the screen. After processing, the route is displayed on the map and the layer will be added to the top of the legend. The Modify Map Layer Properties button can be used to change the line color, style, and width. Use the Remove Active Map Layer button to remove this layer from the map display.

\subsection{MAP LEGEND}

The map legend appears on the left side of the map display. Whenever WebTRAGIS is started, the default map layers for each transportation mode are displayed. Any additional map layers used in previous sessions will not be shown. Also if any of the default layers were removed in a previous session, they will be redisplayed when the model is restarted. The user needs to use the Add Map Layer button to add other user specific or commonly used map layers.

Each layer can easily be shown or removed from the map display by checking or removing the check from the box to the left of the layer name. For example, if the Indian layer showing Native American Tribal Lands is not desired on the map, just remove the check from this layer in the legend.

The order of the map layers can be changed by moving the pointing device over a layer and holding down a mouse button and drag the layer to another position in the legend. Order is important because layers are displayed over another, with the lowest layer in the legend drawn first and the top layer drawn last. So if the States layer is moved to the top of the legend, other layers will appear to have disappeared because the fill color of the States layer will be covering everything.

At the lower portion of the map legend area is the Automatically Add Detail as Zoom option. This feature can be checked for the highway mode of WebTRAGIS. By default, only the Interstate highway are drawn and the U.S. highways and other highways layers are not shown. When this option is checked, as the map is zoomed in, the U.S. highways layer and then the other highways layer will automatically be displayed on the map.

\subsection{STATUS BAR}

Along the bottom edge of the Route Map screen is an area call the status bar, which is comprised of four items. From left to right they are the map display ratio scale, coordinate display, MapTips, and clock display. 


\subsubsection{Map Display Ratio Scale}

The leftmost item in the status bar is the map display ratio scale. The ratio scale is a representative fraction of the ratio between the map and the ground distance. Please note that the WebTRAGIS map display has a geographic Cartesian projection. This type of projection is not the best for comparing distances or showing true area representation, but lends itself to rapid drawing of maps. It also is useful for easily reporting latitude/longitude coordinates.

\subsubsection{Coordinate Display}

The second item in the status bar is the coordinate display. The longitude (X coordinate) and latitude (Y coordinate) of the location of the pointing device on the map is displayed in this area of the status bar. The coordinates are reported as decimal degrees. This display can be useful for adding point symbols at known latitude/longitude locations.

\subsubsection{MapTips}

The third item in the status bar is a check box titled MapTips and two associated drop-down lists. Checking this box allows information to be displayed associated with the select map layer and attribute field. The left drop-down list allows selection of any of the current map layers shown in the legend. The second drop-down list allows selection of any the attribute fields for the selected map layer. For example, check the MapTips box and select the node layer of the transportation network from the first drop-down list. Next select the name field from the second drop-down list. Move the point over a node symbol on the map display and then hold the cursor still. After a brief pause, a box will appear below the pointing device that shows the name of the node. This function can be used for any map layer and attribute field, but works best if the map is zoomed into a portion of a state.

\subsubsection{Clock Display}

The rightmost item in the status bar is a display showing the current time on the client computer. 
Page intentionally left blank 


\section{FUTURE DEVELOPMENT}

The TRAGIS model provides a major change in technology from the earlier routing models developed at ORNL. The HIGHWAY and INTERLINE models were developed at a time when text-based programs were the standard form. With the development of TRAGIS, improved GUI and GIS features are now available to enhance and improve routing model capabilities. Additional improvements will be made to TRAGIS to improve and enhance the performance of the model.

If users have any comments and questions about suggested improvements to the TRAGIS model, please contact the authors. 
Page intentionally left blank 


\section{REFERENCES}

Ardila-Coulson, Maria V. 1989. The Statewide Radioactive Materials Transportation Plan, Phase II, University of Nevada-Reno, Reno, Nevada.

Armstrong, John H. 1998. The Railroad: What It Is, What It Does, Simmons-Boardman Books, Inc., Omaha, Nebraska.

Bhaduri, Budhendra, Edward Bright, Phillip Coleman, and Jerome Dobson, 2002. "LandScan, Locating People is What Matters," GeoInformatics, April/May 2002, pp 34-37.

DOC 2002. Distances Between United States Ports, $2002\left(9^{\text {th }}\right)$ Edition, U.S. Department of Commerce, Washington, D.C.

DOE 2002. Final Environmental Impact Statement for a Geologic Repository for the Disposal of Spent Nuclear Fuel and High-Level Radioactive Waste at Yucca Mountain, Nye County, Nevada, DOE/EIS-0250, U.S. Department of Energy, Washington, D.C.

Johnson, P. E., D. S. Joy, D. B. Clarke, and J. M. Jacobi, 1993a. HIGHWAY 3.1-An Enhanced Highway Routing Model: Program Description, Methodology, and Revised User's Manual, ORNL/TM-12124, Oak Ridge National Laboratory, Oak Ridge, Tennessee.

Johnson, P. E., D. S. Joy, D. B. Clarke, and J. M. Jacobi, 1993b, INTERLINE 5.0-An Expanded Railroad Routing Model: Program Description, Methodology, and Revised User's Manual, ORNL/TM-12090, Oak Ridge National Laboratory, Oak Ridge, Tennessee.

Neuhauser, K. S., and F. L., Kanipe, 2000. RADTRAN 5 Users Guide, SAND2000-1257, Sandia National Laboratories, Albuquerque, New Mexico.

Yuan, Y.C., S.Y. Chen, B.M. Biwer, and D.J. LePoire, 1995. RISKIND—A Computer Program for Calculating Radiological Consequences and Health Risks from Transportation of Spent Nuclear Fuel, ANL/EAD-1, Argonne National Laboratory, Argonne, Illinois. 
Page intentionally left blank 
APPENDIX A

SELECTED FACILITY LOCATIONS IN THE TRAGIS HIGHWAY NETWORK 



\section{APPENDIX A. SELECTED FACILITY LOCATIONS IN THE TRAGIS HIGHWAY NETWORK}

The following list of facility locations provides the appropriate TRAGIS highway node name that should be used in the model. Each location is either identified by a specific node name or the nearest appropriate node name that should be used for routing to that site.

\section{Alabama}

Anniston Army Depot

Bellefonte Nuclear Power Plant (never completed)

Browns Ferry Nuclear Power Plant

Joseph M. Farley Nuclear Power Plant

Marshall Space Flight Center

Maxwell Air Force Base

Port of Mobile, Alabama State Docks

Redstone Arsenal

\author{
ANNISTON DEPOT \\ BELLEFONTE NP \\ BROWNS FERRY NP \\ FARLEY NP \\ MARSHALL SFC \\ MXF AIRPORT \\ AL STATE DOCKS \\ REDSTONE ARSNL N I565S255
}

DMA AIRPORT

LUF AIRPORT

PALO VERDE NP

U OF ARIZONA

YUMA PRVNG GND

\section{California}

Beale Air Force Base

Concord Naval Weapons Station

Diablo Canyon Nuclear Power Station

Edwards Air Force Base

Energy Technology Engineering Center

General Electric Vallecitos Nuclear Center

General Atomics

Humboldt Bay Nuclear Power Plant

Laboratory for Energy-Related Health Research

Lawrence Berkeley National Laboratory

Lawrence Livermore National Laboratory (East Gate)

Lawrence Livermore Site 300

March Air Reserve Base

Moffett Field Naval Air Station

North Island Naval Air Station

Port of Long Beach

BAB AIRPORT
CONCORD NWS
DIABLO CANYON NP
EDW AIRPORT
ETEC
GE VALLECITOS
GENERAL ATOMICS
HUMBOLDT BAY NP
LEHR
L BERKELEY LAB
L LIVERMORE LB
L LIV LB S 300
RIV AIRPORT
NUQ AIRPORT
NZY AIRPORT
PORT OF LONG BEACH


Port of Los Angeles

Port of Oakland

Port of Richmond

Port of Sacramento

Port of San Francisco

Port of Stockton

Rancho Seco Nuclear Power Plant

Rocketdyne Propulsion and Power

San Diego Unified Port

San Onofre Nuclear Power Plant

Sandia National Labs-Livermore (West Gate)

Sierra Army Depot

Sanford Linear Accelerator Center

Travis Air Force Base

UCLA Lab of Nuclear Medicine \& Rad. Biology

University of California, Irvine

Vandenberg Air Force Base

\section{Colorado}

Denver Federal Center

Fort St Vrain Nuclear Power Plant

National Renewable Energy Laboratory

Rocky Flats Plant

Rust Geotech, Grand Junction Project Office

\section{Connecticut}

Haddam Neck Nuclear Power Plant

Knolls Atomic Power Laboratory, Windsor Site

Millstone Nuclear Power Plant

UNC Naval Products

Yale University

\section{Delaware}

Dover Air Force Base

Port of Wilmington

\section{District of Columbia}

DOE/HQ Forrestal Building

\section{Florida}

Canaveral Port Authority

Cape Canaveral Air Force Station

Cecil Field Naval Air Station

Crystal River Nuclear Power Plant

Eglin Air Force Base

Jacksonville Port Authority, Blount Island Terminal

Kennedy Space Center

MacDill Air Force Base

NASA Vehicle Assembly Building

Patrick Air Force Base
PORT OF LA

PORT OF OAKLAND

PORT OF RICHMOND

PORT OF SACMTO

PORT OF SF

PORT OF STOCKTON

RANCHO SECO NP

ROCKETDYNE

PORT OF SAN DIEGO

SAN ONOFRE NP

SNL LIVERMORE

AHC AIRPORT

STANFORD L AC

SUU AIRPORT

UCLA LNM\&RB

U OF C IRVINE

VANDENBERG AFB

DENVER FED CTR

FT ST VRAIN NP

NREL

ROCKY FLATS

GRAND JCT PO

HADDAM NECK NP

KAPL-WINDSOR

MILLSTONE NP

UNC NAVAL

YALE UNIV

DOVER AF BASE

PORT OF WILMINGTON

DOE FORRESTAL

PORT CANAVERAL

C CANAVERAL AFS

CECIL FLD NAS

CRYSTAL RIVER NP

VPS AIRPORT

JACKSONVL PORT

KENNEDY SP CTR

MCF AIRPORT

NASA V A BLDG

COF AIRPORT 
Port Everglades Authority

Port of Miami

St Lucie Nuclear Power Plant

Tampa Port Authority

Turkey Point Nuclear Power Plant

Tyndall Air Force Base

University of Florida

\section{Georgia}

Brunswick State Docks

Dobbins Air Reserve Base

Fort Benning

Georgia Institute of Technology

Edwin I. Hatch Nuclear Power Plant

Kings Bay Naval Submarine Base

Moody Air Force Base

Robins Air Force Base

Port of Savannah

Vogtle Nuclear Power Plant

\section{Idaho}

Argonne National Laboratory West

Idaho National Engineering \& Environmental Lab

Idaho State University

INEEL Chemical Plant

INEEL Radioactive Waste Management Center

Mountain Home Air Force Base

Naval Reactors Site at INEEL

\section{Illinois}

Allied Chemical

Argonne National Laboratory

Braidwood Nuclear Power Plant

Byron Nuclear Power Plant

Clinton Nuclear Power Plant

Dresden Nuclear Power Plant

Fermi National Accelerator Laboratory

General Electric Morris Operations

Illinois International Port, Chicago

LaSalle Nuclear Power Plant

Quad Cities Nuclear Power Plant

Scott Air Force Base

University of Illinois

Zion Nuclear Power Plant

\section{Indiana}

Newport Army Ammunition Plant

Purdue University
PORT EVERGLADES

PORT OF MIAMI

ST LUCIE NP

PORT OF TAMPA

TURKEY POINT NP

PAM AIRPORT

U OF FLORIDA

BRUNSWICK ST DOCKS

MGE AIRPORT

FORT BENNING

GEORGIA TECH

HATCH NP

KINGS BAY NSB

VAD AIRPORT

WRB AIRPORT

SAVANNAH PORT

VOGTLE NP

ARGONNE WEST

INEEL

IDAHO ST UNIV

INEEL CHEM PLT

RWMC

MUO AIRPORT

NAVAL REACTORS

NEWPORT AM PLT

PURDUE UNIV 
Iowa

Ames Laboratory

Duane Arnold Nuclear Power Plant

Iowa State University

\section{Kansas}

McConnell Air Force Base

Kansas State University

Wolf Creek Nuclear Power Plant

\section{Kentucky}

Lexington-Blue Grass Army Depot

Paducah Gaseous Diffusion Plant

\section{Louisiana}

Barksdale Air Force Base

Greater Baton Rouge Port Commission

Port of Lake Charles

Port of New Orleans

River Bend Nuclear Power Plant

Waterford Nuclear Power Plant

\section{Maine}

Maine Yankee Nuclear Power Plant

Port of Portland

Portsmouth, NH Naval Shipyard

\section{Maryland}

Aberdeen Proving Grounds

Andrews Air Force Base

Calvert Cliffs Nuclear Power Plant

Dundalk Marine Terminal, Baltimore

DOE/HQ Germantown

Edgewood Arsenal

National Institute of Standards \& Technology

National Naval Medical Center

University of Maryland

\section{Massachusetts}

Massachusetts Institute of Technology

Massachusetts Port Authority

University of Lowell

University of Massachusetts

Westover Air Reserve Base

Worcester Polytechnic Institute

Yankee-Rowe Nuclear Power Plant

\section{Michigan}

Big Rock Point Nuclear Power Plant

D. C. Cook Nuclear Power Plant
AMES LAB

ARNOLD NP

IOWA ST UNIV

IAB AIRPORT

KANSAS ST UNIV S

WOLF CREEK NP

BLUE GRASS DPO

PADUCAH GDP

BAD AIRPORT

PORT ALLEN S I10 X153

PORT OF LK CHRL

PORT OF NEW ORLEANS

RIVER BEND NP

WATERFORD NP

MAINE YANKEE NP

PORTLAND PORT

PORTSMOUTH NAV

ABERDEEN PRG G

ADW AIRPORT

CALVERT CLIFFS NP

DUNDALK PORT

DOE GERMANTOWN

EDGEWOOD ARSNL

NIST

NAVAL MED CNTR

U OF MARYLAND

MIT

MASSPORT

UNIV OF LOWELL

NORTHAMPTON NE I91 X19

CEF AIRPORT

WORCESTER POLY

YANKEE-ROWE NP

BIG ROCK POINT NP

COOK NP 
Detroit/Wayne County Port Authority

Dow Chemical Plant

Fermi Nuclear Power Plant

Michigan State University

Palisades Nuclear Power Plant

University of Michigan

\section{Minnesota}

Monticello Nuclear Power Plant

Prairie Island Nuclear Power Plant

Seaway Port Authority of Duluth

University of Minnesota

\section{Mississippi}

Columbus Air Force Base

Grand Gulf Nuclear Power Plant

Keesler Air Force Base

National Space Technology Laboratories

Port of Gulfport

Port of Pascagoula

\section{Missouri}

Callaway Nuclear Power Plant

Honeywell Kansas City Plant

University of Missouri

University of Missouri-Rolla

Whiteman Air Force Base

\section{Montana}

Malstrom Air Force Base

\section{Nebraska}

Cooper Station Nuclear Power Plant

Fort Calhoun Nuclear Power Plant

Offutt Air Force Base

U.S. Veterans Hospital, Omaha

\section{Nevada}

Nellis Air Force Base

Nevada Test Site

Tonopah Test Range

Yucca Mountain Repository Site

\section{New Hampshire}

Portsmouth Naval Shipyard

Seabrook Nuclear Power Plant

\section{New Jersey}

Earle Naval Weapons Station

Hope Creek Nuclear Power Plant
PORT OF DETROIT

DOW CHEMICAL

FERMI NP

MICH ST UNIV

PALISADES NP

U OF MICHIGAN

MONTICELLO NP

PRAIRIE ISLAND NP

PORT OF DULUTH

U OF MINNESOTA

CBM AIRPORT

GRAND GULF NP

BIX AIRPORT

NATL S T LAB

PORT OF GULFPORT

PORT OF PASCAGOULA

CALLAWAY NP

K C PLANT

U OF MISSOURI

U OF MO-ROLLA

WHITEMAN AFB

GFA AIRPORT

COOPER NP

FORT CALHOUN NP

OFF AIRPORT

VETERANS HOSP

LSV AIRPORT

MERCURY

TNX AIRPORT

YUCCA MOUNTAIN

(see Maine)

SEABROOK NP

EARLE NWS

HOPE CREEK NP 
McGuire Air Force Base

Oyster Creek Nuclear Power Plant

Paulsboro Terminal

Petty Island Terminal (Port of Philadelphia)

Picatinny Arsenal

Port of Elizabeth

Port of Newark

Princeton University Plasma Physics Laboratory

Salem Nuclear Power Plant

\section{New Mexico}

Alternate Control Facility

Cannon Air Force Base

Holloman Air Force Base

Kirtland Air Force Base

Los Alamos National Laboratory

Lovelace Inhalation Toxicology Research Institute

Sandia National Laboratories

University of New Mexico

Waste Isolation Pilot Plant

White Sands Missile Range

\section{New York}

Brookhaven National Laboratory

Cintichem

Cornell University

Environmental Measurement Laboratory (New York)

James A. FitzPatrick Nuclear Power Plant

Ginna Nuclear Power Plant

Indian Point Nuclear Power Plant

Knolls Atomic Power Laboratory-Kesselring Site

Knolls Atomic Power Laboratory-Knolls Site

Manhattan College

Nine Mile Point Nuclear Power Plant

Port of Buffalo

Rensselaer Polytechnic Institute

SUNY Buffalo

West Valley Demonstration Project

\section{North Carolina}

Brunswick Nuclear Power Plant

General Electric Nuclear Energy Products

Shearon Harris Nuclear Power Plant

McGuire Nuclear Power Plant

North Carolina Port Authority

North Carolina State University

Pope Air Force Base

Port of Wilmington

Seymour Johnson Air Force Base

Sunny Point Marine Terminal
WRI AIRPORT

OYSTER CREEK NP

PORT OF PAULSBORO

PETTY ISL TERM

PICATINNY ARNL

PORT OF ELIZABETH

PORT NEWARK

PRINCTN PLASMA

SALEM NP

\section{ACF}

CVS AIRPORT

HMN AIRPORT

HMN AIRPORT

LOS ALAMOS N L

LOVELACE ITRI

SANDIA NATL LABS

U OF NEW MEXICO

WIPP

WSD AIRPORT

BROOKHAVEN LAB

CINTICHEM NUCL

CORNELL UNIV

ENV MEAS LAB

FITZPATRICK NP

GINNA NP

INDIAN POINT NP

KAPL-KESSELRNG

KAPL-KNOLLS

MANHATTAN COL

NINE MILE POINT NP

PORT OF BUFFALO

RPI

SUNY BUFFALO

WEST VALLEY RP

BRUNSWICK NP

GE NUCLEAR

HARRIS NP

MCGUIRE NP

MOREHEAD CITY

NC STATE UNIV

POB AIRPORT

PORT OF WILMINGTON

GSB AIRPORT

SUNNY POINT TM 


\section{North Dakota}

Grand Forks Air Force Base

Minot Air Force Base

RDR AIRPORT

MINOT AFB

\section{Ohio}

Battelle Memorial Institute

BATTELLE

Battelle West Jefferson Site

BATTELLE W-J

Cleveland-Cuyahoga County Port Authority

CLEVELAND PORT

Davis-Besse Nuclear Power Plant

DAVIS-BESSE NP

Fernald Environmental Management Project

Miamisburg Environmental Management Project

FERNALD PLANT

MOUND FACILITY

Ohio State University

Perry Nuclear Power Plant

OHIO STATE U

PERRY NP

Portsmouth Gaseous Diffusion Plant

PORTSMOUTH GDP

RMI Titanium Company

Toledo-Lucas County Port Authority

RMI PLANT

PORT OF TOLEDO

Wright-Patterson Air Force Base

FFO AIRPORT

\section{Oklahoma}

Altus Air Force Base

LTS AIRPORT

Tinker Air Force Base

TIK AIRPORT

University of Oklahoma

U OF OKLAHOMA

Vance Air Force Base

END AIRPORT

\section{Oregon}

Oregon State University

Port of Portland

Reed College

Trojan Nuclear Power Plant

Umatilla Army Depot

OREGON ST UNIV

PORT OF PORTLAND

REED COLLEGE

TROJAN NP

UMATILLA DEPOT

\section{Pennsylvania}

Beaver Valley Nuclear Power Plant

Bettis Atomic Power Laboratory

Limerick Nuclear Power Plant

Peach Bottom Nuclear Power Plant

Pennsylvania State University

Pittsburgh Energy Technology Center

Pittsburgh Naval Reactors Office

Port of Chester (Eddystone)

Port of Marcus Hook

Port of Philadelphia

Susquehanna Nuclear Power Plant

Three Mile Island Nuclear Power Plant

BEAVER VALLEY NP

BETTIS APL

LIMERICK NP

PEACH BOTTOM NP

PENN STATE

PETC

BETTIS APL

PORT OF CHESTER

PORT OF MARCUS HOOK

PHILADELPHIA PORT

SUSQUEHANNA NP

THREE MILE IS NP

\section{Rhode Island}

Rhode Island AEC

RHODE IS AEC 


\section{South Carolina}

Catawba Nuclear Power Plant

Charleston Air Force Base

Charleston Naval Base

Oconee Nuclear Power Plant

Port of Charleston (N. Charleston Terminal)

Port of Charleston (Wando Terminal)

H. B. Robinson Nuclear Power Plant

Savannah River Site (3/700 Area)

Savannah River Site (MFFF Area)

Savannah River Site (OWPF Area)

Savannah River Site (RBOF Area)

Shaw Air Force Base

Summer Nuclear Power Plant

University of South Carolina

Westinghouse Electric Nuclear Fuel (Columbia)

\section{South Dakota}

Ellsworth Air Force Base

\section{Tennessee}

East Tennessee Technology Park (K-25 Site)

High Flux Isotope Reactor

Nuclear Fuel Services

Oak Ridge National Laboratory

Oak Ridge Reservation

Scientific Ecology Group (Oak Ridge)

Sequoyah Nuclear Power Plant

Solid Waste Area Group 6 (Oak Ridge)

Watts Bar Nuclear Power Plant

Y-12 Plant

\section{Texas}

Camp Bullis

Comanche Peak Nuclear Power Plant

Dyess Air Force Base

Kelly Air Force Base

Laughlin Air Force Base

LBJ Space Flight Center

Mormon Plant

Pantex Plant

Port of Beaumont

Port of Corpus Christi

Port of Freeport

Port of Galveston

Port of Houston

Port of Port Arthur

Port of Texas City

Randolph Air Force Base

South Texas Nuclear Power Plant
CATAWBA NP

CHARLESTON AFB

CHARLESTON NAVAL BASE

OCONEE NP

N CHARLTN TERM

WANDO TERMINAL

ROBINSON NP

SRS

SRS SITE F

SRS SITE S

SRS SITE H

SSC AIRPORT

SUMMER NP

U OF S CAROLINA

WEST NF FAB

RCA AIRPORT

$\mathrm{K}-25$

HFIR

NFS ERWIN

ORNL

O R RSVTN SITE

SEG

SEQUOYAH NP

SWAG-6

WATTS BAR NP

$\mathrm{Y}-12$

CAMP BULLIS

COMANCHE PEAK NP

DYS AIRPORT

SKF AIRPORT

DLF AIRPORT

LBJ SPACE CNTR

MORMON PLANT

PANTEX PLANT

PORT OF BEAUMONT

PORT OF CORPUS CHR

PORT OF FREEPORT

PORT OF GALVSTN

PORT OF HOUSTON

PORT ARTHUR PT

PORT OF TEXAS CITY

RANDOLPH AFB

SOUTH TEXAS NP 
Texas A\&M University

University of Texas

Utah

Dugway Proving Grounds

Envirocare

Safety-Kleen Grayback Mountain Facility

Tooele Army Depot

University of Utah

\section{Vermont}

Vermont Yankee Nuclear Power Plant

\section{Virginia}

ARECO Facility

Babcock \& Wilcox, Lynchburg

Langley Air Force Base

Newport News Marine Terminal

Norfolk International Terminal

Norfolk Naval Air Station

Norfolk Naval Base

North Anna Nuclear Power Plant

Port of Richmond

Portsmouth Marine Terminal

Portsmouth Naval Shipyard

Surry Nuclear Power Plant

University of Virginia

Yorktown Naval Weapons Station

\section{Washington}

Bangor Undersea Warfare Engineering Station Annex

Columbia Nuclear Power Plant

Fairchild Air Force Base

Hanford Site (Westinghouse Hanford, Pacific NW Lab)

McCord Air Force Base

Port of Keyport

Port of Seattle

Port of Tacoma

Puget Sound Naval Shipyard (Bremerton)

University of Washington

Washington State University

\section{West Virginia}

Morgantown Energy Technology Center

\section{Wisconsin}

Kewaunee Nuclear Power Plant

LaCrosse Nuclear Power Plant

Point Beach Nuclear Power Plant

University of Wisconsin
TEXAS A\&M UNIV

U OF TEXAS

DUGWAY PVG GND

CLIVE

GRAYBACK FCLTY

TOOELE DEPOT

U OF UTAH

VERMONT YANKEE NP

FOREST

B\&W FUEL PLANT

LFI AIRPORT

N N MARINE TERM

NORFOLK INTL TM

NGU AIRPORT

NORFOLK NAVAL BASE

NORTH ANNA NP

VA DEEPWTR TERM

PM MARINE TERM

PM NAVAL SHPYRD

SURRY NP

U OF VIRGINIA

YORKTOWN NWS

BANGOR UWESA

COLUMBIA NP

SKA AIRPORT

HANFORD

TCM AIRPORT

KEYPORT

PORT OF SEATTLE

PORT OF TACOMA

PS NAVAL SHPYD

U OF WASHINGTON

WASHINGTON ST

METC

KEWAUNEE NP

LA CROSSE NP

POINT BEACH NP

U OF WISCONSIN 
Wyoming

Francis E. Warren Air Force Base

F E WARREN AFB

A-12 
APPENDIX B

TRAGIS RAILROAD NETWORK ABBREVIATION 



\section{APPENDIX B. TRAGIS RAILROAD NETWORK ABBREVIATION}

This appendix consists of two tables. Table B-1 provides a list of the abbreviations in the TRAGIS Railroad Network Version 2.0. The first column provides the abbreviation of the 97 different rail systems and the right column identifies the individual railroads within each system. The first three systems $(<\mathrm{C} 3>$, $<\mathrm{OR}>$, and $<\mathrm{TR}>$ ) comprise a large number of small railroad companies, which are not individually identified. Most of the other entries provide the full name of the primary railroad, the holding or parent company, and the other railroads companies and their reporting markings.

For example, the ARZC is the abbreviation for the Arizona \& California Railroad. RailAmerica, Inc. owns this railroad. Other railroads in the ARZC system within the TRAGIS rail network include the Arizona Eastern Railway, San Diego \& Imperial Valley Railroad, San Joaquin Valley Railroad, San Pedro \& Southwestern Railway, and Ventura County Railroad.

Table B-2 provides a cross reference between all the individual railroad companies and the respective rail system in which each company is located in the TRAGIS rail network. The first column is the railroad abbreviation found in the rail ownership or trackage rights attribute fields in the rail network. The second column identifies the TRAGIS rail system to which the railroad is assigned. This table is rather large (over 800 entries) and is provided primarily to aid users who need to lookup railroad companies and their respective rail system assignment.

Table B-1. The 97 rail systems in the TRAGIS rail network.

$<$ C3 $>\quad$ Other Class III (Shortline) Railroads

$<\mathrm{OR}>\quad$ Other Railroads (non carriers, commuter, museum, tourist, etc.)

$<$ TR $>\quad$ Terminal Railroads

ACWR Aberdeen, Carolina \& Western Railway

AM Arkansas \& Missouri Railroad

AMTK Amtrak (National Railroad Passenger Corporation)

ARZC Arizona \& California Railroad (RailAmerica, Inc.) also Arizona Eastern Railway (AZER); San Diego \& Imperial Valley Railroad (SDIY); San Joaquin Valley Railroad (SJVR); San Pedro \& Southwestern Railway (SWKR); Ventura County Railroad (VCRR)

BAR Bangor \& Aroostook Railroad; also Canadian American Railroad (CDAC)

BAYL Bay Line Railroad (Rail Management Corp.) also Copper Basin Railway (CBRY); Galveston Railway (GVSR); Kentucky West Tennessee Railway (KWT); Little Rock \& Western Railway (LRWN); M \& B Railroad (MNBR); Tomahawk Railway (TR); Western Kentucky Railway (WKRL)

BCLR Bay Colony Railroad also Seminole Gulf Railway (SGLR)

BLE Bessemer \& Lake Erie Railroad (Great Lakes Transportation)

BLMR Blue Mountain Railroad (Watco Companies) also Eastern Idaho Railroad (EIRR); Palouse River \& Coulee City Railroad (PCC)

BNSF Burlington Northern Santa Fe Railway

BPRR Buffalo \& Pittsburgh Railroad (Genesee \& Wyoming Inc.) also Genesee \& Wyoming Railroad (GNWR), Illinois \& Midland Railroad (IMRR), Louisiana \& Delta Railroad (LDRR), Rochester \& Southern Railroad (RSR)

BRC Belt Railway of Chicago 
CAGY Columbus \& Greenville Railway also Chattooga \& Chickamauga Railway (CCKY);

Luxapalila Valley Railroad (LXVR); Redmont Railway (RRC)

CFNR California Northern (RailAmerica, Inc.)

CN Canadian National Railway

CORP Central Oregon \& Pacific Railroad (RailAmerica, Inc.)

CPRS Canadian Pacific Railway

CR Conrail Shared Assets (jointly owned by CSXT and NS)

CSS Chicago, Southshore \& South Bend Railroad (Anacostia \& Pacific) also Louisville \& Indiana Railroad (LIRC); Pacific Harbor Lines (PHL)

CSXT CSX Transportation

CVR Cimarron Valley Railroad (Western Group) also Arizona Central Railroad (AZCR); Southwestern Railroad (SW); Wyoming \& Colorado Railroad (WYCO)

DME Dakota, Minnesota \& Eastern Railroad

DMIR Duluth, Missabe \& Iron Range Railway (Great Lakes Transportation)

DMVW Dakota, Missouri Valley \& Western Railroad

DSRC Dakota Southern Railway

DSRR Delta Southern Railroad

EJE Elgin, Joliet \& Eastern Railway (Transtar) also Birmingham Southern Railroad (BS); Lake Terminal Railroad (LT), McKeesport Connecting Railroad (MKC); Union Railroad (URR)

ELS Escanaba \& Lake Superior Railroad

FEC Florida East Coast Railway

FMRC Farmrail (Farmrail Systems) also Finger Lakes Railway (FGLK); Grainbelt Corporation (GNVC)

FWWR Fort Worth \& Western Railroad

GC Georgia Central (Rail Management Corp.) also Atlantic \& Western Railway (ATW); East Tennessee Railway (ETRY); Valdosta Railway (VR); Wilmington Terminal Railroad (WTRY)

GFRR Georgia \& Florida RailNet (Gulf \& Ohio Railways) also H \& S Railroad (HS); Knoxville \& Holston River Railroad (KXHR); Lexington \& Ohio River Railroad (LXOH); Mississippi Delta Railroad (MSDR); Nash County Railroad (NCYR); Wiregrass Central Railroad (WGCR); Yadkin Valley Railroad (YVRR)

GSWR Georgia Southwestern Railroad

GWR Great Western Railway (OmniTrax) also Council Bluffs Railway (CBGR); Chicago Rail Link (CRL); Georgia Woodlands Railroad (GWRC); Manufacturers Junction Railway (MJ); Northern Ohio \& Western Railway (NOW); Newburgh \& South Shore Railroad (NSR); Panhandle Northern Railroad (PNR)

HESR Huron and Eastern Railway (RailAmerica, Inc.) also Grand Rapids Eastern Railway (GR); Mid-Michigan Railroad (MMRR); Michigan Shore Railroad (MS); Saginaw Valley Railway (SGVY)

HOG Heart of Georgia Railroad

HRRC Housatonic Railroad

IAIS Iowa Interstate Railroad

IHB Indiana Harbor Belt Railroad

IMRL I \& M Rail Link

INPR Idaho Northern \& Pacific Railroad (Rio Grande Pacific Corp.)

INRD Indiana Railroad

IORY Indiana \& Ohio Railway (RailAmerica, Inc.) also Central Railroad Company of Indianapolis (CERA); Central Railroad Company of Indiana (CIND); Indiana \& Ohio Central Railroad 
(IOCR)

ISRR Indiana Southern Railroad (RailAmerica, Inc.)

KBSR Kankakee, Beaverville \& Southern Railroad

KCS Kansas City Southern Railway also Texas Mexican Railway (TM)

KO Kansas \& Oklahoma Railroad

KRR Kiamichi Railroad (RailAmerica, Inc.) also Dallas Garland \& Northeastern Railroad (DGNO); Texas Northeastern Railroad (TNER)

KYLE Kyle Railroad (RailAmerica, Inc.)

LAL Livonia Avon \& Lakeville Railroad also Oil Creek \& Titusville Lines (OCTL); Ontario Central Railroad (ONCT); Western New York \& Pennsylvania Railroad (WNYP)

LSRC Lake State Railway

MHWA Mohawk Adirondack \& Northern Railroad (Genesee Valley Transportation) also Delaware \& Lackawanna Railway (DL); Depew Lancaster \& Western Railroad (DLWR); Falls Road Railroad (FRR); Lowville \& Beaver River Railroad (LBR)

MNA Missouri \& Northern Arkansas Railroad (RailAmerica, Inc.) also Otter Tail Valley Railroad (OTVR)

MNN Minnesota Northern Railroad also St Croix Valley Railroad (SCXY)

MRL Montana Rail Link

MSCI Mississippi Central Railroad (Pioneer Railcorp) also Alabama \& Florida Railway (AF); Alabama Railway (ALAB); Decatur Junction Railway (DT); Fort Smith Railway (FSR); Garden City Western Railway (GCW); Gettysburg \& Northern Railroad (GET); Indiana Southwestern Railway (ISW); Keokuk Junction Railway (KJRY); Michigan Southern Railroad (MSO); Pioneer Industrial Railway (PRY); Shawnee Terminal Railway (STR); Vandalia Railroad (VRRC); West Michigan Railroad (WMI)

NCRC Nebraska Central (Rio Grande Pacific Corp.) also New Orleans \& Gulf Coast Railway (NOGC); Wichita Tillman \& Jackson Railway (WTJR)

NECR New England Central Railroad (RailAmerica, Inc.) also Connecticut Southern Railroad (CSO)

NERR Nashville \& Eastern Railway (South Central Rail Group) also Nashville \& Western Railroad (NWR); Tennken Railroad (TKEN); West Tennessee Railroad (WTNN)

NKCR Nebraska Kansas \& Colorado RailNet (North American RailNet) also Camas Prairie Railnet (CSP); Illinois Railnet (IR); Mississippi \& Tennessee Railnet (MT)

NPR Northern Plains Railroad

NS Norfolk Southern Railway

NSHR North Shore Railroad also Juniata Valley Railroad (JVRR); Lycoming Valley Railroad (LVRR); Nittany \& Bald Eagle Railroad (NBER); Stourbridge Railroad (SBRR); Shamokin Valley Railroad (SVRR); Union County Industrial Railroad (UCIR); Wellsboro \& Corning Railroad (WCOR)

NWPY Northwestern Pacific Railway

NYA New York \& Atlantic Railway (Anacostia \& Pacific)

NYSW New York, Susquehanna \& Western Railway

OHCR Ohio Central Railroad also Columbus \& Ohio River Railroad (CUOH); Mahoning Valley Railway (MVRY); Ohio \& Pennsylvania Railroad (OHPA); Ohio Southern Railroad (OSRR); Pittsburgh \& Ohio Central Railroad (POHC); Warren \& Trumbull Railroad (WTRM); Youngstown \& Austintown Railroad (YARR); Youngstown Belt Railroad (YB)

PAL Paducah \& Louisville Railway

PSAP Puget Sound \& Pacific Railroad (RailAmerica, Inc.) also Cascade \& Columbia Railroad (CSCD)

PVRR Pioneer Valley Railroad (Pinsly Railroad Co.) also Arkansas Midland Railroad (AKMD); 
Florida Central Railroad (FCEN); Florida Midland Railroad (FMID); Florida Northern Railroad (FNOR)

PW Providence \& Worchester Railroad

RBMN Reading Blue Mountain Railroad

RJCR R J Corman Railroad also R J Corman/Cleveland Line (RJCL); R J Corman/Memphis Line (RJCM); R J Corman/Allentown Lines (RJCN); R J Corman/Pennsylvania Lines (RJCP); R J Corman/Western Ohio Line (RJCW)

RRVW Red River Valley \& Western Railroad

SCRF South Carolina Central Railroad (RailAmerica, Inc.) also Alabama \& Gulf Coast Railway (AGR); Chesapeake \& Albemarle Railroad (CA); Carolina Piedmont Division/SCRF (CPDR); Eastern Alabama Railway (EARY); North Carolina \& Virginia Railroad (NCVA); Virginia Southern Division/NCVA (VSRR)

SCXF South Central Florida Express

SKOL South Kansas \& Oklahoma Railroad (Watco Companies) also Kansas Eastern Railroad (KE); Osage Railroad (ORR); Stillwater Central Railroad (SLWC); Timber Rock Railroad (TIBR)

SLR St Lawrence \& Atlantic Railroad (Genesee \& Wyoming Inc.) also Penn Eastern Rail Lines (PRL); York Railway (YRC)

ST $\quad$ ST Rail System (Guilford Rail System)

TCWR Twin Cities \& Western Railroad also Minnesota Central Railroad (MCTA)

TNMR Texas \& New Mexico Railroad (Permian Basin Railways, Inc.) also West Texas \& Lubbock Railroad (WTLR)

TPW Toledo, Peoria \& Western Railway (RailAmerica, Inc.)

TRRA Terminal Railroad Association of St Louis

TSBY Tuscola \& Saginaw Bay Railway

TXNW Texas North Western Railway (TNW Corp.) also Nebraska Northeastern Railway (NENE); Texas Gonzales \& Northern Railway (TXGN); Texas Rock Crusher Railway (TXR)

TXPF Texas Pacifico Transportation

UP Union Pacific Railroad

USG United States Government

UTAH Utah Railway

VTR Vermont Railway also Clarendon \& Pittsford Railroad (CLP); Green Mountain Railroad (GMRC); New York \& Ogdensburg Railway (NYOG)

WE Wheeling \& Lake Erie Railway also Akron Barberton Cluster Railway (AB)

WPRR Willamette \& Pacific Railroad (Genesee \& Wyoming Inc.) also Portland \& Western Railroad (PNWR)

WSOR Wisconsin Southern Railroad 
Table B-2. Cross reference between railroad company and rail system.

\begin{tabular}{|c|c|c|c|}
\hline Railroad company & TRAGIS network & AYR & $<\mathrm{OR}>$ \\
\hline $\mathrm{AA}$ & $<\mathrm{C} 3>$ & AZCR & CVR \\
\hline$A B$ & WE & AZER & ARZC \\
\hline$A B L$ & $<\mathrm{TR}>$ & BAR & BAR \\
\hline ABR & $<\mathrm{C} 3>$ & BAYL & BAYL \\
\hline$A C$ & $\mathrm{CN}$ & BB & $<\mathrm{C} 3>$ \\
\hline ACE & $<\mathrm{OR}>$ & BCLR & BCLR \\
\hline ACJR & $<\mathrm{C} 3>$ & $\mathrm{BCOL}$ & $<\mathrm{OR}>$ \\
\hline ACWR & ACWR & BCRY & $<\mathrm{OR}>$ \\
\hline ADBF & $<$ C3 $>$ & BCS & $<\mathrm{OR}>$ \\
\hline ADSR & $<\mathrm{OR}>$ & BDRV & $<\mathrm{C} 3>$ \\
\hline AERC & $<\mathrm{C} 3>$ & BDTL & $<\mathrm{TR}>$ \\
\hline $\mathrm{AF}$ & $\mathrm{MSCl}$ & BDW & $<\mathrm{C} 3>$ \\
\hline AFR & $<\mathrm{C} 3>$ & BEEM & $<\mathrm{C} 3>$ \\
\hline AGCR & $<\mathrm{C} 3>$ & BFRD & $<\mathrm{OR}>$ \\
\hline AGR & SCRF & $\mathrm{BH}$ & $<\mathrm{C} 3>$ \\
\hline AKDN & $<\mathrm{C} 3>$ & $\mathrm{BHC}$ & $<\mathrm{OR}>$ \\
\hline AKMD & PVRR & $\mathrm{BHP}$ & $<\mathrm{C} 3>$ \\
\hline $\mathrm{AL}$ & $<\mathrm{C} 3>$ & BHWY & $<\mathrm{C} 3>$ \\
\hline ALAB & $\mathrm{MSCl}$ & BIRR & $<$ C3 $3>$ \\
\hline ALM & $<\mathrm{C} 3>$ & BJRY & $<\mathrm{TR}>$ \\
\hline ALQS & $<$ C3 $>$ & BKRR & $<\mathrm{C} 3>$ \\
\hline ALS & $<\mathrm{TR}>$ & BLE & BLE \\
\hline AM & AM & BLMR & BLMR \\
\hline AMTK & AMTK & BLOL & $<\mathrm{C} 3>$ \\
\hline AN & $<\mathrm{C} 3\rangle$ & BLR & $<\mathrm{C} 3>$ \\
\hline ANR & $<$ C3 $>$ & $\mathrm{BMH}$ & $<$ C3 $>$ \\
\hline ANY & $<\mathrm{OR}>$ & BML & $<\mathrm{C} 3>$ \\
\hline AOK & $<\mathrm{C} 3>$ & BMLP & $<\mathrm{OR}>$ \\
\hline APA & $<C 3>$ & BNML & BNSF \\
\hline APD & $<$ TR $>$ & BNSF & BNSF \\
\hline APNC & $<C 3>$ & BOCT & CSXT \\
\hline AR & $<\mathrm{C} 3>$ & BOP & $<\mathrm{C} 3>$ \\
\hline ARA & $<$ C3 $>$ & BPRR & BPRR \\
\hline ARC & $<$ C3 $>$ & BRAN & $<\mathrm{C} 3>$ \\
\hline ARE & $<\mathrm{C} 3\rangle$ & BRC & $\mathrm{BRC}$ \\
\hline ARN & $<\mathrm{OR}>$ & BRG & $<\mathrm{TR}>$ \\
\hline ARR & $<\mathrm{OR}>$ & BRW & $<\mathrm{C} 3>$ \\
\hline ARZC & ARZC & BS & EJE \\
\hline ASR & $<\mathrm{C} 3\rangle$ & BSDA & $<\mathrm{C} 3>$ \\
\hline ASRY & $<$ C3 $>$ & BSFS & $<\mathrm{OR}>$ \\
\hline ATLT & $<\mathrm{C} 3>$ & BSOR & $<\mathrm{C} 3>$ \\
\hline ATW & GC & BST & $<\mathrm{OR}>$ \\
\hline AUAR & $<$ C3 $>$ & BSVR & $<$ C3 $>$ \\
\hline AVR & $<\mathrm{C} 3>$ & BVRY & $<$ C3 $3>$ \\
\hline AWW & $<\mathrm{C} 3>$ & BXN & $<C 3>$ \\
\hline
\end{tabular}




\begin{tabular}{|c|c|c|c|}
\hline CA & SCRF & CNOW & $<$ C3 $>$ \\
\hline CAGY & CAGY & CNUR & $<\mathrm{C} 3>$ \\
\hline CALA & $<$ C3 $3>$ & CNZR & $<\mathrm{TR}>$ \\
\hline CATS & $<\mathrm{OR}>$ & COEH & $<C 3>$ \\
\hline CBC & $<\mathrm{OR}>$ & COER & $<C 3>$ \\
\hline CBGR & GWR & $\mathrm{COP}$ & $<$ C3 $>$ \\
\hline CBL & $<\mathrm{C} 3>$ & CORP & CORP \\
\hline CBNS & $<\mathrm{OR}>$ & CPDR & SCRF \\
\hline CBRM & $<\mathrm{C} 3>$ & CPRS & CPRS \\
\hline CBRW & $<\mathrm{C} 3>$ & CR & CR \\
\hline CBRY & BAYL & CRL & GWR \\
\hline $\mathrm{CcC}$ & $<\mathrm{OR}>$ & CRLE & $<C 3>$ \\
\hline CCKY & CAGY & CS & $<\mathrm{OR}>$ \\
\hline CCPN & $<\mathrm{TR}>$ & CSCD & PSAP \\
\hline CCRA & $<C 3>$ & CSKR & $<C 3>$ \\
\hline CCRR & $<$ C3 $>$ & CSL & $<\mathrm{TR}>$ \\
\hline CCT & $<\mathrm{C} 3>$ & CSO & NECR \\
\hline CCUO & $<\mathrm{TR}>$ & CSP & NKCR \\
\hline CDAC & BAR & CSRR & $<\mathrm{OR}>$ \\
\hline CDTX & $<O R>$ & CSS & CSS \\
\hline CEIW & $<\mathrm{C} 3>$ & CSXT & CSXT \\
\hline CEMR & $<\mathrm{OR}>$ & CT & $<\mathrm{TR}>$ \\
\hline CERA & IORY & CTN & $<\mathrm{TR}>$ \\
\hline CF & $<\mathrm{C} 3>$ & CTR & $<$ C3 $>$ \\
\hline CFNR & CFNR & CTRW & $<\mathrm{OR}>$ \\
\hline CFWR & $<\mathrm{C} 3>$ & CUMB & $<\mathrm{OR}>$ \\
\hline CGR & $<\mathrm{OR}>$ & $\mathrm{CUOH}$ & OHCR \\
\hline $\mathrm{CHR}$ & $<\mathrm{C} 3>$ & CUVA & $<\mathrm{TR}>$ \\
\hline CHRR & $<\mathrm{C} 3>$ & CVR & CVR \\
\hline CHTT & $<\mathrm{C} 3>$ & CVSR & $<\mathrm{OR}>$ \\
\hline $\mathrm{Cl}$ & $<\mathrm{C} 3\rangle$ & CVYR & $<$ C3 $>$ \\
\hline $\mathrm{CIC}$ & $<\mathrm{C} 3>$ & $\mathrm{CW}$ & $<$ C3 $>$ \\
\hline CIND & IORY & CWCY & $<$ C $3>$ \\
\hline CIR & $<C 3>$ & CWR & $<C 3>$ \\
\hline CIRR & $<\mathrm{C} 3>$ & CWRL & $<\mathrm{OR}>$ \\
\hline CIRY & $<\mathrm{C} 3>$ & CWRY & $<\mathrm{C} 3>$ \\
\hline CKP & $<\mathrm{C} 3>$ & CZRY & $<$ C3 $>$ \\
\hline CKSI & $<\mathrm{OR}>$ & DAIR & $<$ C3 $>$ \\
\hline CLC & $<C 3>$ & DAKR & $<C 3>$ \\
\hline CLNA & $<\mathrm{C} 3>$ & DAKS & $<\mathrm{C} 3>$ \\
\hline CLP & VTR & DART & $<\mathrm{OR}>$ \\
\hline CLRT & $<\mathrm{OR}>$ & $\mathrm{DC}$ & $<\mathrm{TR}>$ \\
\hline $\mathrm{CM}$ & $<\mathrm{C} 3>$ & DCFB & $<C 3>$ \\
\hline CMGN & $<$ C3 $>$ & DCLR & $<$ C3 $>$ \\
\hline CMPA & $<\mathrm{C} 3>$ & DCRR & $<\mathrm{C} 3>$ \\
\hline CMSL & $<\mathrm{OR}>$ & DGNO & $\mathrm{KRR}$ \\
\hline CMTN & $<\mathrm{OR}>$ & DKS & $<$ C3 $>$ \\
\hline $\mathrm{CN}$ & $\mathrm{CN}$ & $\mathrm{DL}$ & MHWA \\
\hline CNAT & $<\mathrm{OR}>$ & DLWR & MHWA \\
\hline
\end{tabular}




\begin{tabular}{|c|c|c|c|}
\hline DME & DME & FGC & $<\mathrm{OR}>$ \\
\hline DMIR & DMIR & FGLK & FMRC \\
\hline DMVW & DMVW & FLWB & $<\mathrm{OR}>$ \\
\hline DNE & $<\mathrm{C} 3\rangle$ & FMID & PVRR \\
\hline DQE & $<C 3>$ & FMRC & FMRC \\
\hline DR & $<\mathrm{C} 3\rangle$ & FNOR & PVRR \\
\hline DRHY & $<\mathrm{C} 3\rangle$ & $\mathrm{FP}$ & $<$ C3 $>$ \\
\hline DRIR & $<$ C3 $>$ & FRR & MHWA \\
\hline DRM & $<\mathrm{OR}>$ & FRVT & $<\mathrm{C} 3\rangle$ \\
\hline DSNG & $<\mathrm{OR}>$ & FSR & $\mathrm{MSCl}$ \\
\hline DSRC & DSRC & FSRR & $<\mathrm{OR}>$ \\
\hline DSRR & DSRR & FVRR & $<$ C $3>$ \\
\hline DT & $\mathrm{MSCl}$ & FVW & $\mathrm{CN}$ \\
\hline DURR & $<\mathrm{OR}>$ & FWCR & $<\mathrm{C} 3\rangle$ \\
\hline DV & $<\mathrm{C} 3\rangle$ & FWDB & $<\mathrm{TR}>$ \\
\hline DVR & $<\mathrm{OR}>$ & FWPP & $<\mathrm{OR}>$ \\
\hline DVS & $<\mathrm{C} 3>$ & FWRY & $<\mathrm{C} 3>$ \\
\hline DW & $<\mathrm{OR}>$ & FWWR & FWWR \\
\hline $\mathrm{EACH}$ & $<\mathrm{C} 3\rangle$ & FXE & $<C 3\rangle$ \\
\hline EARY & SCRF & GC & $\mathrm{GC}$ \\
\hline EASO & $<\mathrm{C} 3\rangle$ & GCRY & $<\mathrm{OR}>$ \\
\hline ECBR & $<C 3>$ & GCSR & $<$ C $3>$ \\
\hline ECO & $<\mathrm{OR}>$ & GCW & $\mathrm{MSCl}$ \\
\hline ECTB & $<\mathrm{C} 3\rangle$ & GET & $\mathrm{MSCl}$ \\
\hline EDW & $<$ C3 $>$ & GEXR & $<\mathrm{OR}>$ \\
\hline EE & $<$ C3 $>$ & GFR & $<\mathrm{OR}>$ \\
\hline EEC & $<\mathrm{TR}>$ & GFRR & GFRR \\
\hline EFRR & $<$ C $3>$ & GGS & SCRF \\
\hline EIRC & $<\mathrm{C} 3>$ & GITM & $<$ C3 $>$ \\
\hline EIRR & BLMR & GLSR & $<$ C3 $>$ \\
\hline EJE & EJE & GMLG & $<\mathrm{OR}>$ \\
\hline EJR & $<\mathrm{TR}\rangle$ & GMRC & VTR \\
\hline ELKR & $<\mathrm{C} 3\rangle$ & GMRY & $<$ C3 $>$ \\
\hline ELS & ELS & GNBC & FMRC \\
\hline EMHR & $<$ C3 $>$ & GNRR & $<$ C $3>$ \\
\hline ENR & $<\mathrm{OR}>$ & GNWR & BPRR \\
\hline EPRY & $<$ C3 $>$ & GR & HESR \\
\hline EPTC & $<\mathrm{C} 3>$ & GRC & $<\mathrm{OR}>$ \\
\hline ESHR & $<\mathrm{C} 3\rangle$ & GRIV & $<$ C $3>$ \\
\hline ETER & $<\mathrm{OR}>$ & GRR & $<$ C $3>$ \\
\hline ETL & $<\mathrm{OR}>$ & GRWR & $<C 3>$ \\
\hline ETRY & GC & GSM & $<$ C3 $>$ \\
\hline EV & $<\mathrm{C} 3>$ & GSWR & GSWR \\
\hline EWR & $\mathrm{MSCl}$ & GTD & $<\mathrm{OR}>$ \\
\hline F\&W & $<\mathrm{OR}>$ & GTR & $<$ C3 $>$ \\
\hline FC & $<C 3>$ & GTRA & $<\mathrm{TR}>$ \\
\hline FCCM & $<\mathrm{OR}>$ & GU & $<$ C3 $>$ \\
\hline FCEN & PVRR & GVSR & BAYL \\
\hline FEC & FEC & GWR & GWR \\
\hline
\end{tabular}




\begin{tabular}{|c|c|c|c|}
\hline GWRC & GWR & JEFW & $<T R>$ \\
\hline GWRS & $<O R>$ & $\mathrm{JKL}$ & $<\mathrm{C} 3>$ \\
\hline GWWE & KCS & JSRC & $<\mathrm{OR}>$ \\
\hline GWWR & KCS & JVRR & NSHR \\
\hline $\mathrm{HB}$ & $<\mathrm{C} 3>$ & KBSR & KBSR \\
\hline HBRY & $<\mathrm{OR}>$ & $\operatorname{KCCX}$ & $<\mathrm{OR}>$ \\
\hline HCRR & $<\mathrm{TR}>$ & KCDX & $<\mathrm{TR}>$ \\
\hline HCRY & $<\mathrm{OR}>$ & KCS & KCS \\
\hline $\mathrm{HE}$ & $<\mathrm{C} 3>$ & $\mathrm{KCT}$ & $<\mathrm{TR}>$ \\
\hline HESR & HESR & $\mathrm{KE}$ & SKOL \\
\hline HLSC & $<\mathrm{C} 3>$ & KJR & $<$ C3 $>$ \\
\hline HMCR & $<$ C3 $>$ & KJRY & $\mathrm{MSCl}$ \\
\hline $\mathrm{HN}$ & $<\mathrm{C} 3>$ & KKRR & $<$ C3 $>$ \\
\hline HOBO & $<\mathrm{OR}>$ & KNOR & $<$ C3 $>$ \\
\hline HOG & HOG & $\mathrm{KO}$ & $\mathrm{KO}$ \\
\hline HOS & $<$ C $3>$ & KPR & $<\mathrm{OR}>$ \\
\hline HPTD & $<$ C3 $>$ & KRL & $<\mathrm{OR}>$ \\
\hline HRRC & HRRC & $\mathrm{KRR}$ & KRR \\
\hline HRS & $<$ C3 $>$ & KRT & $<\mathrm{OR}>$ \\
\hline HRT & $<\mathrm{C} 3>$ & KSRY & $<\mathrm{C} 3>$ \\
\hline $\mathrm{HS}$ & GFRR & $\mathrm{KT}$ & $<$ C3 $>$ \\
\hline HSRR & $<\mathrm{C} 3>$ & KTR & $\mathrm{MSCl}$ \\
\hline HUCK & $<\mathrm{OR}>$ & KWT & BAYL \\
\hline HVR & $<O R>$ & KXHR & GFRR \\
\hline HVSR & $<\mathrm{OR}>$ & KYLE & KYLE \\
\hline IAIS & IAIS & LAJ & $<\mathrm{TR}>$ \\
\hline IANR & $<\mathrm{C} 3>$ & LAL & LAL \\
\hline IANW & $<$ C3 $>$ & LBR & MHWA \\
\hline IATR & $<\mathrm{TR}>$ & LC & $<\mathrm{C} 3>$ \\
\hline IBT & $<T R>$ & LCR & $<\mathrm{OR}>$ \\
\hline ICRK & $<$ C3 $>$ & LCSR & $<\mathrm{OR}>$ \\
\hline IHB & $\mathrm{IHB}$ & LDRR & BPRR \\
\hline IHCR & $<\mathrm{OR}>$ & LEF & $<\mathrm{OR}>$ \\
\hline IHRR & $<\mathrm{OR}>$ & LER & $<C 3>$ \\
\hline ILW & $<$ C3 $>$ & LFCD & $<\mathrm{OR}>$ \\
\hline IMRL & IMRL & LHRR & $<\mathrm{C} 3>$ \\
\hline IMRR & BPRR & LINC & $<C 3>$ \\
\hline IN & $<\mathrm{C} 3>$ & LIRC & CSS \\
\hline INPR & INPR & LIRR & $<\mathrm{OR}>$ \\
\hline INRD & INRD & LKRR & $<\mathrm{C} 3>$ \\
\hline IOCR & IORY & LMIC & $<$ C3 $>$ \\
\hline IORY & IORY & LNAL & $<\mathrm{C} 3>$ \\
\hline IR & NKCR & LNVT & $<$ C3 $>$ \\
\hline IRRS & $<\mathrm{OR}>$ & LNW & $<\mathrm{C} 3>$ \\
\hline ISR & $<$ C $3>$ & LPN & $<$ C3 $>$ \\
\hline ISRR & ISRR & LR & $<\mathrm{OR}>$ \\
\hline ISSR & $<\mathrm{C} 3>$ & LRPA & $<\mathrm{TR}>$ \\
\hline ISW & $\mathrm{MSCl}$ & LRS & $<$ C3 $>$ \\
\hline ITM & $<\mathrm{OR}>$ & LRWN & BAYL \\
\hline
\end{tabular}




\begin{tabular}{|c|c|c|c|}
\hline LRY & $<\mathrm{OR}>$ & MNNR & $<\mathrm{TR}>$ \\
\hline LS & $<$ C3 $>$ & MOC & $<\mathrm{C} 3>$ \\
\hline LSI & $<$ C $3>$ & MPP & $<\mathrm{OR}>$ \\
\hline LSRC & LSRC & MRD & $<\mathrm{OR}>$ \\
\hline LT & EJE & MRI & $<C 3>$ \\
\hline LTV & $<\mathrm{OR}>$ & MRL & MRL \\
\hline LUN & $<\mathrm{C} 3>$ & MRS & $<T R>$ \\
\hline LVRC & $<$ C3 $>$ & MS & HESR \\
\hline LVRR & NSHR & $\mathrm{MSCl}$ & $\mathrm{MSCl}$ \\
\hline LW & $<$ C3 $>$ & MSDR & GFRR \\
\hline $\mathrm{LXOH}$ & GFRR & MSE & $<\mathrm{C} 3>$ \\
\hline LXVR & CAGY & MSN & $<\mathrm{C} 3>$ \\
\hline MAA & $<\mathrm{C} 3>$ & MSO & $\mathrm{MSCl}$ \\
\hline MARC & $<\mathrm{OR}>$ & MSRW & $<\mathrm{C} 3>$ \\
\hline MAW & $<C 3>$ & MSTR & $<C 3>$ \\
\hline MBRX & $<\mathrm{OR}>$ & MSV & $<\mathrm{C} 3>$ \\
\hline MBTA & $<\mathrm{OR}>$ & MSWY & $<\mathrm{C} 3>$ \\
\hline MC & $<\mathrm{OR}>$ & MT & NKCR \\
\hline MCER & $<\mathrm{C} 3>$ & MTDB & $<\mathrm{OR}>$ \\
\hline MCLR & $<$ C3 $>$ & MVRY & $\mathrm{OHCR}$ \\
\hline MCR & $<$ C3 $>$ & MVT & $<\mathrm{TR}>$ \\
\hline MCRM & $<\mathrm{OR}>$ & MWCL & $<C 3>$ \\
\hline MCRR & $<\mathrm{TR}>$ & MWRC & $<\mathrm{OR}>$ \\
\hline MCSA & $<\mathrm{C} 3>$ & MWRR & $<\mathrm{C} 3>$ \\
\hline MCTA & TCWR & NAUG & $<\mathrm{C} 3>$ \\
\hline MDDE & $<$ C3 $>$ & NBEC & $<\mathrm{OR}>$ \\
\hline MDLR & $<\mathrm{TR}>$ & NBER & NSHR \\
\hline MDS & $<$ C3 $>$ & NBSR & $<\mathrm{OR}>$ \\
\hline MDW & $<\mathrm{TR}>$ & NCPR & $<T R>$ \\
\hline ME & $<C 3>$ & NCR & $<\mathrm{OR}>$ \\
\hline MET & $<\mathrm{TR}>$ & NCRC & NCRC \\
\hline METR & $<\mathrm{OR}>$ & NCRY & $<$ C $3>$ \\
\hline METW & $<$ C3 $>$ & NCVA & SCRF \\
\hline MGRI & $<\mathrm{TR}>$ & NCYR & GFRR \\
\hline $\mathrm{MH}$ & $<$ C3 $>$ & NDCR & $<$ C3 $>$ \\
\hline MHWA & MHWA & NDM & $<$ C3 $>$ \\
\hline MIDH & $<$ C3 $>$ & NDT & $<\mathrm{OR}>$ \\
\hline MJ & GWR & NECR & NECR \\
\hline MKC & EJE & NEGS & $<\mathrm{C} 3>$ \\
\hline MLO & $<\mathrm{OR}>$ & NENE & TXNW \\
\hline MMF & $<\mathrm{OR}>$ & NERR & NERR \\
\hline MMID & $<\mathrm{C} 3>$ & $\mathrm{NHCR}$ & $<\mathrm{C} 3>$ \\
\hline MMRR & HESR & $\mathrm{NHN}$ & $<\mathrm{C} 3>$ \\
\hline MNA & MNA & NHRR & $<C 3>$ \\
\hline MNBR & BAYL & NHVS & $<\mathrm{OR}>$ \\
\hline MNCW & $<\mathrm{OR}>$ & NHVT & $<\mathrm{C} 3>$ \\
\hline MNG & $<\mathrm{OR}>$ & NJT & $<\mathrm{OR}>$ \\
\hline MNJ & $<\mathrm{C} 3>$ & NKCR & NKCR \\
\hline MNN & MNN & NMGR & $<\mathrm{C} 3>$ \\
\hline
\end{tabular}




\begin{tabular}{|c|c|c|c|}
\hline NOGC & NCRC & OTVR & MNA \\
\hline NOKL & $<$ C3 $>$ & $\mathrm{OUCH}$ & $<$ C3 $>$ \\
\hline NOPB & $<\mathrm{TR}>$ & PAL & PAL \\
\hline NOW & GWR & PAM & $<\mathrm{TR}>$ \\
\hline NPB & $<\mathrm{TR}>$ & PATH & $<\mathrm{OR}>$ \\
\hline NPR & NPR & PBL & $<\mathrm{TR}>$ \\
\hline NPS & $<\mathrm{OR}>$ & PBNE & $<$ C3 $>$ \\
\hline NRI & $<\mathrm{C} 3\rangle$ & PBR & $<\mathrm{C} 3>$ \\
\hline NRR & $<\mathrm{C} 3\rangle$ & PBRR & $<\mathrm{C} 3>$ \\
\hline NS & NS & PBVR & $<$ C3 $>$ \\
\hline NSHR & NSHR & PCC & BLMR \\
\hline NSM & $<O R>$ & PCN & $<$ C3 $>$ \\
\hline NSR & GWR & PDMX & $<\mathrm{C} 3\rangle$ \\
\hline NSSR & $<O R>$ & PDRR & $<$ C3 $>$ \\
\hline NTRY & $<\mathrm{TR}\rangle$ & PGR & $<\mathrm{C} 3>$ \\
\hline NVRR & $<$ C3 $>$ & $\mathrm{PHL}$ & CSS \\
\hline NWPY & NWPY & $\mathrm{PI}$ & $<\mathrm{TR}>$ \\
\hline NWR & NERR & PICK & $<\mathrm{C} 3>$ \\
\hline NYA & NYA & PJR & $<$ C3 $>$ \\
\hline $\mathrm{NYCH}$ & $<\mathrm{TR}>$ & PJRL & $<\mathrm{C} 3>$ \\
\hline NYGL & $<$ C3 $>$ & PKHP & $<$ C3 $>$ \\
\hline NYLE & $<\mathrm{C} 3>$ & PLV & $<\mathrm{TR}>$ \\
\hline NYOG & VTR & PNR & GWR \\
\hline NYSW & NYSW & PNW & $<$ C3 $>$ \\
\hline OAR & $<$ C3 $>$ & PNWR & WPRR \\
\hline OCN & $<O R>$ & $\mathrm{POHC}$ & $\mathrm{OHCR}$ \\
\hline OCRR & $<\mathrm{OR}>$ & РОТВ & $<\mathrm{TR}>$ \\
\hline OCTL & LAL & POVA & $<$ C3 $>$ \\
\hline OGEE & $<$ C3 $>$ & PPU & $<\mathrm{TR}>$ \\
\hline OHCR & OHCR & PRL & SLR \\
\hline $\mathrm{OHIC}$ & $<$ C3 $>$ & PRT & $<\mathrm{TR}>$ \\
\hline OHPA & OHCR & PRV & $<\mathrm{C} 3>$ \\
\hline OHRY & $<$ C3 $>$ & PRY & $\mathrm{MSCl}$ \\
\hline OKAN & $<\mathrm{OR}>$ & PRYL & $<$ C3 $>$ \\
\hline OLB & $<$ C3 $>$ & PSAP & PSAP \\
\hline OLO & $<O R>$ & $\mathrm{PT}$ & $<\mathrm{TR}>$ \\
\hline OLYR & $<$ C3 $>$ & PTC & $<$ C3 $>$ \\
\hline OMID & $<$ C3 $>$ & PTR & $<\mathrm{TR}>$ \\
\hline ONCT & LAL & PTRA & $<\mathrm{TR}>$ \\
\hline ONT & $<\mathrm{OR}>$ & PTRC & $<\mathrm{TR}>$ \\
\hline OPE & $<$ C3 $>$ & PUCC & $<\mathrm{TR}>$ \\
\hline OPT & $<T R>$ & PVRR & PVRR \\
\hline ORR & SKOL & PVS & $<$ C3 $>$ \\
\hline OSCV & $<\mathrm{OR}>$ & PVTX & $<\mathrm{OR}>$ \\
\hline OSRR & $\mathrm{OHCR}$ & PW & PW \\
\hline отсо & $<$ C3 $>$ & QBT & $<\mathrm{TR}>$ \\
\hline OTCR & $<\mathrm{OR}>$ & QCR & $<\mathrm{OR}>$ \\
\hline OTR & $<\mathrm{TR}>$ & QGRY & $<\mathrm{OR}>$ \\
\hline OTT & $<T R>$ & QRC & $<\mathrm{OR}>$ \\
\hline
\end{tabular}




\begin{tabular}{|c|c|c|c|}
\hline QRR & $<C 3>$ & SEI & $<\mathrm{TR}>$ \\
\hline RARW & $<$ C3 $>$ & SEPA & $<\mathrm{OR}>$ \\
\hline RBMN & RBMN & SERA & $<\mathrm{C} 3>$ \\
\hline RCBT & $<\mathrm{OR}>$ & SFLR & $<$ C3 $>$ \\
\hline RCRR & $<\mathrm{OR}>$ & SFR & $<$ C3 $>$ \\
\hline RCRY & $<$ C3 $>$ & SFS & $<C 3>$ \\
\hline RJCL & RJCR & SGLR & BCLR \\
\hline RJCM & RJCR & SGVY & HESR \\
\hline RJCN & RJCR & $\mathrm{SH}$ & $<\mathrm{TR}>$ \\
\hline RJCP & RJCR & SIND & $<$ C3 $>$ \\
\hline RJCR & RJCR & SIRT & $<\mathrm{OR}>$ \\
\hline RJCW & RJCR & SJVR & ARZC \\
\hline $\mathrm{RL}$ & $<\mathrm{C} 3>$ & SKOL & SKOL \\
\hline RLHH & $<\mathrm{OR}>$ & SL & $<\mathrm{C} 3>$ \\
\hline RLK & $<\mathrm{OR}>$ & SLAL & $<\mathrm{C} 3\rangle$ \\
\hline RMRR & $<$ C3 $>$ & SLC & $<$ C3 $>$ \\
\hline ROR & $<\mathrm{OR}>$ & SLE & $<\mathrm{OR}>$ \\
\hline RRC & CAGY & SLGG & $<\mathrm{C} 3>$ \\
\hline RRRR & $<$ C3 $>$ & SLGW & $<$ C3 $>$ \\
\hline RRVW & RRVW & SLQ & $<\mathrm{OR}>$ \\
\hline RS & $<\mathrm{OR}>$ & SLR & SLR \\
\hline RSM & $<\mathrm{TR}>$ & SLRS & $<\mathrm{C} 3>$ \\
\hline RSNR & $<$ C3 $>$ & SLWC & SKOL \\
\hline RSP & $<\mathrm{C} 3>$ & SM & $<\mathrm{C} 3>$ \\
\hline RSR & BPRR & SMA & $<\mathrm{C} 3>$ \\
\hline RSS & $<\mathrm{C} 3>$ & SMNR & $<\mathrm{OR}>$ \\
\hline RT & $<$ TR $>$ & SMR & $<\mathrm{OR}>$ \\
\hline RVPR & $<\mathrm{OR}>$ & SMRR & $<$ C3 $>$ \\
\hline RVSC & $<$ C3 $>$ & SMV & $<\mathrm{C} 3>$ \\
\hline SAN & $<$ C3 $>$ & SNR & $<$ C3 $>$ \\
\hline SAPT & $<\mathrm{TR}>$ & SOM & $<$ C3 $>$ \\
\hline SAW & $<\mathrm{C} 3\rangle$ & SORA & $<\mathrm{OR}>$ \\
\hline SB & $<\mathrm{TR}>$ & SQVR & $<$ C $3>$ \\
\hline SBK & $<\mathrm{C} 3>$ & SRC & $<\mathrm{C} 3\rangle$ \\
\hline SBLN & $<\mathrm{C} 3\rangle$ & SRN & $<C 3>$ \\
\hline SBRR & NSHR & SRNJ & $<\mathrm{C} 3>$ \\
\hline SBVR & $<$ C3 $>$ & SRP & $<\mathrm{C} 3>$ \\
\hline SCAX & $<O R>$ & SRY & $<\mathrm{OR}>$ \\
\hline SCBG & $<C 3>$ & SS & $<\mathrm{TR}>$ \\
\hline SCEG & $<\mathrm{OR}>$ & SSAM & $\mathrm{CN}$ \\
\hline SCRF & SCRF & SSC & $<$ C3 $>$ \\
\hline SCS & $<\mathrm{OR}>$ & SSLV & $<$ C3 $>$ \\
\hline SCTR & $<$ C $3>$ & SSTX & $<\mathrm{OR}>$ \\
\hline SCXF & SCXF & ST & ST \\
\hline SCXY & MNN & STE & $<\mathrm{TR}>$ \\
\hline SDBB & $<\mathrm{C} 3>$ & STMA & $<$ C3 $>$ \\
\hline SDIY & ARZC & STR & $\mathrm{MSCl}$ \\
\hline SDNR & $<\mathrm{OR}>$ & STRT & $<$ C3 $>$ \\
\hline SE & $<\mathrm{C} 3\rangle$ & SUN & $<$ C3 $>$ \\
\hline
\end{tabular}




\begin{tabular}{|c|c|c|c|}
\hline SVRR & NSHR & TTIS & $<\mathrm{C} 3>$ \\
\hline SVTP & $<\mathrm{OR}>$ & TTR & $<\mathrm{TR}>$ \\
\hline SW & CVR & TVA & $<\mathrm{OR}>$ \\
\hline SWKR & ARZC & TVRM & $<\mathrm{OR}>$ \\
\hline SWL & $<\mathrm{OR}>$ & TVRR & $<\mathrm{C} 3>$ \\
\hline SWP & $<$ C3 $>$ & TXGN & TXNW \\
\hline SWPC & $<\mathrm{OR}>$ & TXNW & TXNW \\
\hline SWR & $<\mathrm{OR}>$ & TXOR & $<\mathrm{C} 3>$ \\
\hline SYRR & $<\mathrm{C} 3>$ & TXPF & TXPF \\
\hline TASD & $<T R>$ & TXR & TXNW \\
\hline TBRY & $<C 3>$ & TXU & $<C 3>$ \\
\hline TCB & $<\mathrm{C} 3>$ & TYBR & $<C 3>$ \\
\hline TCG & $<\mathrm{C} 3>$ & UCIR & NSHR \\
\hline TCKR & $<\mathrm{C} 3>$ & UCRY & $<\mathrm{C} 3>$ \\
\hline TCRY & $<\mathrm{OR}>$ & UHRR & $<\mathrm{OR}>$ \\
\hline TCT & $<\mathrm{TR}>$ & UMP & $<\mathrm{TR}>$ \\
\hline TCWR & TCWR & UP & UP \\
\hline TFM & $<\mathrm{C} 3>$ & UPCS & $<\mathrm{OR}>$ \\
\hline TFVM & $<\mathrm{OR}>$ & URR & EJE \\
\hline TI & $<\mathrm{OR}>$ & USG & USG \\
\hline TIBR & SKOL & UTAH & UTAH \\
\hline TIOS & $<\mathrm{OR}>$ & VALE & $<\mathrm{OR}>$ \\
\hline TIRL & $<C 3>$ & VCRR & ARZC \\
\hline TISH & $<\mathrm{C} 3>$ & VMV & $<\mathrm{OR}>$ \\
\hline TKEN & NERR & VR & GC \\
\hline TM & KCS & VRE & $<\mathrm{OR}>$ \\
\hline TMBL & $<\mathrm{C} 3>$ & VRR & $<\mathrm{C} 3>$ \\
\hline TMET & $<\mathrm{OR}>$ & VRRC & $\mathrm{MSCl}$ \\
\hline TMSS & $<\mathrm{C} 3>$ & VSR & $<\mathrm{C} 3>$ \\
\hline TMUS & $<\mathrm{OR}>$ & VSRR & SCRF \\
\hline $\mathrm{TN}$ & $<\mathrm{C} 3>$ & VSUE & $<\mathrm{OR}>$ \\
\hline TNER & $\mathrm{KRR}$ & VTR & VTR \\
\hline TNHR & $<$ C3 $>$ & VVF & $<\mathrm{OR}>$ \\
\hline TNMR & TNMR & WACR & $<\mathrm{C} 3>$ \\
\hline TOR & $<\mathrm{OR}>$ & WBCR & $<\mathrm{C} 3>$ \\
\hline TPW & TPW & WC & $\mathrm{CN}$ \\
\hline TR & BAYL & WCCL & $<\mathrm{TR}>$ \\
\hline TRC & $<\mathrm{C} 3>$ & WCLR & $<$ C3 $>$ \\
\hline TRE & $<\mathrm{OR}>$ & WCOR & NSHR \\
\hline TRIN & $<$ C3 $>$ & WCR & $<\mathrm{OR}>$ \\
\hline TRMW & $<\mathrm{C} 3>$ & WE & WE \\
\hline TRRA & TRRA & WFS & $<\mathrm{C} 3>$ \\
\hline TSBY & TSBY & WGCR & GFRR \\
\hline TSE & $<C 3>$ & WGNS & $<\mathrm{C} 3>$ \\
\hline TSR & $<\mathrm{OR}>$ & WGR & $<C 3>$ \\
\hline TSRD & $<\mathrm{C} 3>$ & WHOE & $<\mathrm{C} 3>$ \\
\hline TSRR & $<C 3>$ & WHRC & $<\mathrm{OR}>$ \\
\hline TSU & $<\mathrm{TR}>$ & WKRL & BAYL \\
\hline TSWR & $<\mathrm{C} 3>$ & WKS & $<\mathrm{OR}>$ \\
\hline
\end{tabular}




\begin{tabular}{|c|c|c|c|}
\hline WMI & $\mathrm{MSCl}$ & WVN & $<\mathrm{C} 3>$ \\
\hline WMSR & $<\mathrm{OR}>$ & WVR & $<\mathrm{C} 3>$ \\
\hline WNFR & $<$ C3 $>$ & WVRR & $<\mathrm{C} 3>$ \\
\hline WNYP & LAL & WW & $<C 3>$ \\
\hline WPRR & WPRR & WWRC & $<\mathrm{C} 3>$ \\
\hline WRM & $<\mathrm{OR}>$ & WWVS & $<\mathrm{OR}>$ \\
\hline WRRC & $<\mathrm{C} 3>$ & WYCO & CVR \\
\hline WS & $<\mathrm{OR}>$ & WYEC & $<\mathrm{C} 3>$ \\
\hline WSOR & WSOR & YARR & OHCR \\
\hline WSR & $<$ C3 $>$ & YB & OHCR \\
\hline WSRY & $<\mathrm{TR}>$ & YDC & $<\mathrm{OR}>$ \\
\hline WSS & $<\mathrm{C} 3>$ & YERM & $<\mathrm{OR}>$ \\
\hline WTCO & $<\mathrm{C} 3>$ & YRC & SLR \\
\hline WTCX & $<\mathrm{OR}>$ & YRPL & $<\mathrm{C} 3>$ \\
\hline WTJR & NCRC & YSLR & $<\mathrm{TR}>$ \\
\hline WTLR & TNMR & YVRR & GFRR \\
\hline WTNN & NERR & YVT & $<\mathrm{C} 3>$ \\
\hline WTRM & OHCR & YW & $<\mathrm{C} 3>$ \\
\hline WTRY & GC & & \\
\hline WVC & $<\mathrm{C} 3>$ & & \\
\hline
\end{tabular}


Page intentionally left blank

B-16 
APPENDIX C

RAIL SHIPMENTS TO YUCCA MOUNTAIN 



\section{APPENDIX C. RAIL SHIPMENTS TO YUCCA MOUNTAIN}

Some users of the TRAGIS model will be examining possible rail routes for spent nuclear fuel shipments between commercial reactors sites and the Yucca Mountain Repository site in Nevada. Currently, the Yucca Mountain site does not have rail access, but the TRAGIS rail network includes all the proposed rail alignments from existing rail lines to Yucca Mountain examined in the FEIS (DOE 2002). All of the proposed alignments are designated as B branch lines and identified as owned by the USG. Transfer nodes are located at the locations were the proposed alignments meet the Union Pacific Railroad owned lines in northern, eastern, and southern Nevada. Figure C.1 shows the TRAGIS rail network with the proposed rail alignments in red, the proposed transfer locations, and the location of Yucca Mountain site and Las Vegas.

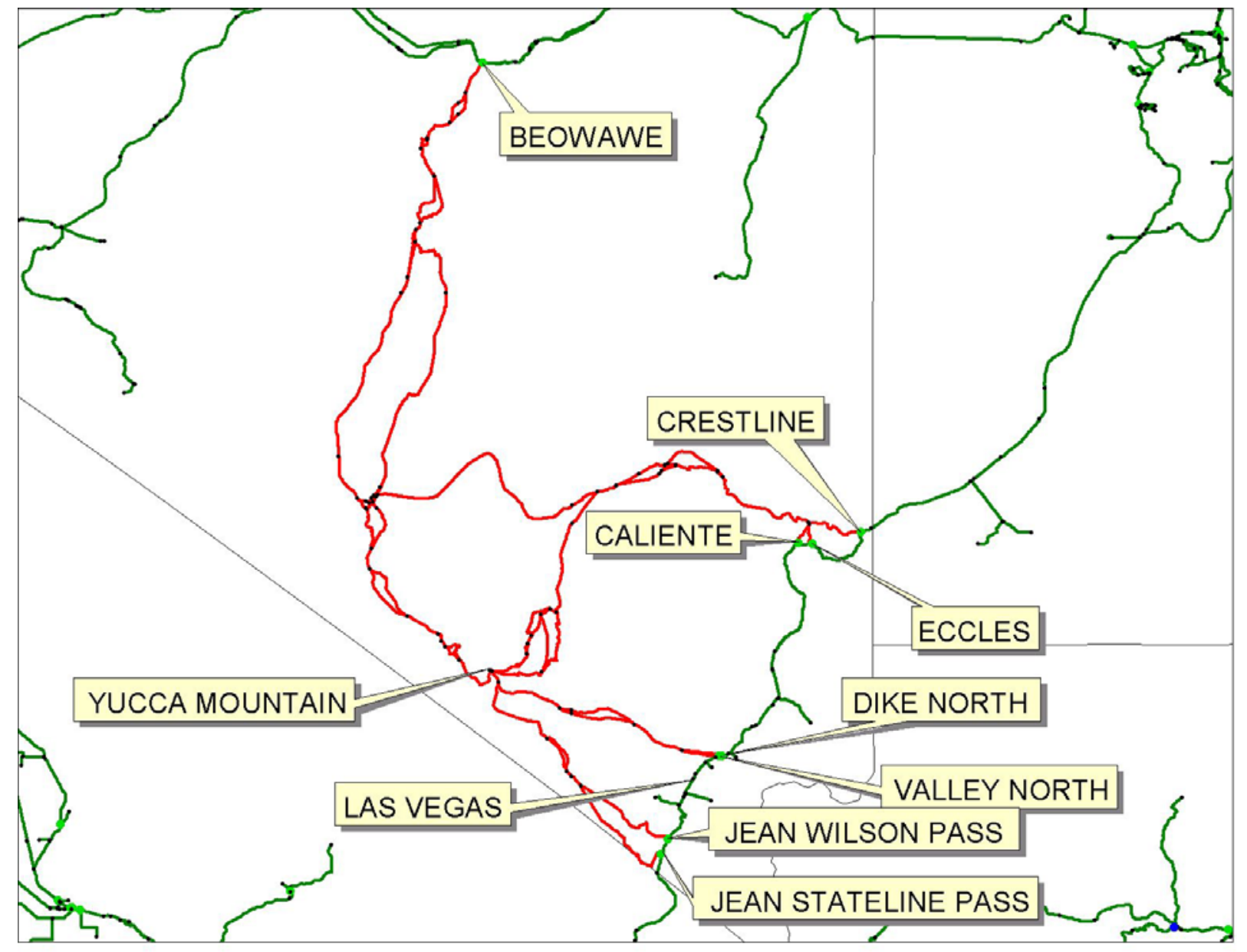

Fig. C.1. Proposed rail alignment to the Yucca Mountain Repository.

By default, all of the possible alignments are open for use. The proposed rail lines will not be used during normal routing unless Yucca Mountain is selected as an origin or destination. When Yucca Mountain is selected, most users will not want all the proposed alignments available. The first step is to block the transfer locations only allowing one access point open. Additionally there are multiple paths for the proposed alignments to Yucca Mountain. Without further action, the model will select the shortest path 
from the open interchange to the Yucca Mountain site. Figure C.1 shows a number of variations between Beowawe and Yucca Mountain. If the user needs to route along a specified set of link, the other adjacent links will need to be selectively blocked.

It is suggested that the following steps be taken for routing to Yucca Mountain.

1. Change the Rail Transfer Penalty for all but one of the transfer locations accessing the Yucca Mountain rail lines to a high value, such as 3000 units. This is accomplished by:

- Click on the Optional Rail/Water Routing Paramters tab.

- Scroll through the Railroad A list and select USG.

- Scroll through the Railroad B list and select UP. A list of transfer locations between the USG and UP rail systems will appear in the Node Name scroll list.

- Select a transfer location that is not desired for use.

- Enter the value of 3000 in the A-B Penalty box.

- Enter the value of 3000 in the B-A Penalty box.

○ Press the Enter New Penalty button.

- Repeat this last step for each of the other transfer locations, except for the location that should be used to access Yucca Mountain.

The transfer locations to access the proposed alignments to Yucca Mountain are, working clockwise from the north:

- Beowawe, NV

- Cresline, NV

- Eccles, NV

- Caliente, NV

- Dike North, NV

- Valley North, NV

- Jean Wilson Pass, NV

- Jean Stateline Pass, NV

2. Block links that are not desired for use. This is accomplished by:

- Click on the Route Map tab and zoom into the area of interest.

- Click on the MapTips function in the lower right portion of the Route Map screen.

- Select RailLink from the first pull-down menu list.

○ Select Link from the second pull-down menu list.

- Move the pointing device over an individual link to determine the link number of the feature. Note the number of the link.

- Click on the Block Nodes/Links tab.

- Enter the link number, followed by a space and then the USG abbreviation (in uppercase), in the Manual Link Entry box in the lower right portion of the Block Links portion of this screen. After typing in the link number and railroad abbreviation (separated by a space), press the enter key. The link number and railroad abbreviation will appear in the Blocked Link Numbers list twice, once followed by the one digit and the other with a negative one (see discussion in Sect. 4.7.2.1).

- Repeat this process until all desired links are blocked.

Please contact the TRAGIS development team, telephone numbers are available by clicking on the Help button on the lower portion of the application screen, if further assistance is needed with rail routing to Yucca Mountain, Nevada. 
APPENDIX D.

SELECTED FACILITY LOCATIONS IN THE TRAGIS RAIL NETWORK 



\section{APPENDIX D. SELECTED FACILITY LOCATIONS IN THE TRAGIS RAIL NETWORK}

The following list of facility locations provides the appropriate TRAGIS rail node name that should be used in the model. Each location is either identified by a specific node name or the nearest appropriate node name that should be used for routing to that site along with the appropriate railroad company that provides service at the site.

\section{Alabama}

Anniston Army Depot

ANNISTON ARMY

NS

Bellefonte Nuclear Power Plant (never completed)

Browns Ferry Nuclear Power Plant

BELLEFONTE NP

NS nearest rail node

no direct rail access

ATHENS

CSXT

Farley Nuclear Power Plant

FARLEY NP

NS

\section{Arizona}

Palo Verde Nuclear Power Plant

PALO VERDE NP

UP

\section{Arkansas}

Arkansas Nuclear One Power Plant

Pine Bluff Arsenal

$\begin{array}{ll}\text { ARKANSAS ONE NP } & \text { UP } \\ \text { PINE BLUFF ARSENAL } & \text { USG }\end{array}$

\section{California}

Concord Naval Weapons Station

Diablo Canyon Nuclear Power Plant nearest rail node

Energy Technology Engineering Center

G E Vallecitos Nuclear Center

Humboldt Bay Nuclear Power Plant

Lawrence Berkeley National Laboratory

Lawrence Livermore National Laboratory

Lawrence Livermore Site 300

Rancho Seco Nuclear Power Plant

Rocketdyne

San Onofre Nuclear Power Plant

Stanford Linear Accelerator Center

CONCORD NWS

UP

no direct rail access

EAST SAN LUIS OBISPO UP

CHATSWORTH

UP

no direct rail access

HUMBOLDT BAY NP NWPY

no direct rail access

no direct rail access

no direct rail access

RANCHO SECO NP

UP

CHATSWORTH

UP

SAN ONOFRE NP

BNSF

\section{Colorado}

Fort St Vrain Nuclear Power Plant

no direct rail access

no direct rail access

no direct rail access

National Renewable Energy Laboratory

ROCKY FLATS

UP

Rush Geotech

no direct rail access

\section{Connecticut}

Haddam Neck (Conn Yankee) Nuc. Po. Plant nearest rail node

Millstone Nuclear Power Plant

no direct rail access

PORTLAND

PW

MILLSTONE NP

PW 


\section{Florida}

Crystal River Nuclear Power Plant nearest rail node

St Lucie Nuclear Power Plant nearest rail node

Turkey Point Nuclear Power Plant nearest rail node

\section{Georgia}

Hatch Nuclear Power Plant

Kings Bay Naval Submarine Base

Vogtle Nuclear Power Plant

\section{Idaho}

Argonne National Laboratory-West

Idaho National Engineering \& Env. Laboratory

\section{Illinois}

Argonne National Laboratory

Braidwood Nuclear Power Plant

Byron Nuclear Power Plant

Clinton Nuclear Power Plant

Dresden Nuclear Power Plant

Fermi National Accelerator Laboratory

G E Morris Operations

La Salle Nuclear Power Plant

Quad Cities Nuclear Power Plant

Zion Nuclear Power Plant

\section{Indiana}

Crane Naval Surface Warfare Center

Newport Army Ammunition Plant

\section{Iowa}

Ames Laboratory

Duane Arnold Nuclear Power Plant

\section{Kansas}

Wolf Creek Nuclear Power Plant

\section{Kentucky}

Lexington-Blue Grass Army Depot

Paducah Gaseous Diffusion Plant

\section{Louisiana}

River Bend Nuclear Power Plant

Waterford Nuclear Power Plant

\section{Maine}

Maine Yankee Nuclear Power Plant no direct rail access

RED LEVEL POWER PLAN CSXT

no direct rail access

FORT PIERCE FEC

no direct rail access

node \#122102597

CSXT

HATCH NP

NS

KINGS BAY

$<$ C3 $>$

NS

no direct rail access

INEEL

UP

$\begin{array}{ll}\text { ARGONNE } & \text { BNSF } \\ \text { BRAIDWOOD NP } & \text { UP } \\ \text { BYRON NP } & \text { IMRL } \\ \text { CLINTON NP } & \text { CN } \\ \text { DRESDEN NP } & \text { EJE } \\ \text { nOde \#172101039 } & \text { BNSF } \\ \text { MORRIS OPERATION } & \text { EJE } \\ \text { LA SALLE NP } & \text { BNSF } \\ \text { QUAD CITIES NP } & \text { IMRL } \\ \text { ZION NP } & \text { UP }\end{array}$

CRANE

CPRS

NEWPORT ARMY AMMUNIT CSXT

no direct rail access

DUANE ARNOLD NP $<$ C3 $>$

WOLF CREEK NP UP

LEXINGTON-BLUE GRASS USG

PADUCAH GDP PAL

RIVER BEND NP CN

WATERFORD NP UP

MAINE YANKEE NP $\quad<$ C3 $>$ 


\section{Maryland}

Calvert Cliffs Nuclear Power Plant nearest rail access

Edgewood Arsenal

no direct rail access

BENEDICT/CHALK POINT CSXT

EDGEWOOD ARSENAL USG

\section{Massachusetts}

Pilgrim Nuclear Power Plant nearest rail access

Yankee-Rowe Nuclear Power Plant nearest rail access

no direct rail access

NORTH PLYMOUTH

BCLR

no direct rail access

node \#252100058

ST

\section{Michigan}

Big Rock Point Nuclear Power Plant nearest rail access

D C Cook Nuclear Power Plant

Fermi Nuclear Power Plant

Palisades Nuclear Power Plant nearest rail access

no direct rail access

node \#26210145

TSBY

DC COOK NP

CSXT

FERMI NP

$\mathrm{CN}$

no direct rail access

HARTFORD

CSXT

\section{Minnesota}

Monticello Nuclear Power Plant

Prairie Island Nuclear Power Plant

MONTICELLO NP

BNSF

PRAIRIE ISLAND NP

CPRS

\section{Mississippi}

Grand Gulf Nuclear Power Plant nearest rail access

no direct rail access

LETOURNEAU

KCS

\section{Missouri}

Allied Signal Aerospace Company

Callaway Nuclear Power Plant nearest rail access

no direct rail access

no direct rail access

FULTON

$\mathrm{KCS}$

\section{Nebraska}

Cooper Nuclear Power Plant nearest rail access

Fort Calhoun Nuclear Power Plant nearest rail access

no direct rail access

node \#312102649

UP

no direct rail access

IA-NE ST LINE UP N

UP

\section{Nevada}

Yucca Mountain Repository (rail access not built yet)

YUCCA MOUNTAIN

USG

\section{New Hampshire}

Seabrook Nuclear Power Plant

SEABROOK NP

\section{New Jersey}

Hope Creek Nuclear Power Plant nearest rail access

Oyster Creek Nuclear Power Plant nearest rail access

no direct rail access

node \#342101778

no direct rail access

LAKEHURST 
Princeton University Plasma Physics Laboratory

Salem Nuclear Power Plant

nearest rail access

\section{New Mexico}

Los Alamos National Laboratory

Lovelace Inhalation Toxicology Research Institute

Sandia National Laboratories

Waste Isolation Pilot Plant

\section{New York}

Brookhaven National Laboratory

FitzPatrick Nuclear Power Plant

Ginna Nuclear Power Plant nearest rail access

Indian Point Nuclear Power Plant nearest rail access

Nine Mile Point Nuclear Power Plant

West Valley Demonstration Project

\section{North Carolina}

Brunswick Nuclear Power Plant

McGuire Nuclear Power Plant

Shearon Harris Nuclear Power Plant

Sunny Point Marine Terminal

\section{Ohio}

Davis-Besse Nuclear Power Plant

Fernald Environmental Management Project

Mound Plant

Perry Nuclear Power Plant

Portsmouth Gaseous Diffusion Plant

\section{Oregon}

Trojan Nuclear Power Plant

Umatilla Army Depot

\section{Pennsylvania}

Beaver Valley Nuclear Power Plant

Bettis Atomic Power Laboratory

Limerick Nuclear Power Plant

Peach Bottom Nuclear Power Plant nearest rail access

Pittsburgh Energy Technology Center

Pittsburgh Naval Reactors Office

Susquehanna Nuclear Power Plant

Three Mile Island Nuclear Power Plant no direct rail access

no direct rail access

node \#342101778

$<\mathrm{C} 3>$

no direct rail access

no direct rail access

SANDIA NATL LABS USG

WIPP

BNSF

BROOKHAVEN NATL LAB

NYA

FITZPATRICK NP

CSXT

no direct rail access

node \#342101778

no direct rail access

node \#362103677

$<\mathrm{C} 3>$

NINE MILE POINT NP

WEST VALLEY BPRR

BRUNSWICK NP USG

MCGUIRE NP CSXT

SHEARON HARRIS NP CSXT

SUNNY POINT MARINE T USG

$\begin{array}{ll}\text { DAVIS-BESSE NP } & \text { NS } \\ \text { FERNALD } & \text { CSXT } \\ \text { MIAMISBURG } & \text { NS } \\ \text { PERRY NP } & \text { NS } \\ \text { PORTSMOUTH GDP } & \text { CSXT }\end{array}$

TROJAN NP

WPRR

ORDNANCE UP

BEAVER VALLEY NP CSXT

no direct rail access

LIMERICK NP

NS

no direct rail access

STEWARTSTOWN $\quad<$ C3 $>$

node \#422104282 CSXT

no direct rail access

SUSQUEHANNA NP NSHR

THREE MILE ISLAND NP NS

CATAWBA NP

NS

\section{South Carolina}

Catawba Nuclear Power Plant 
Oconee Nuclear Power Plant nearest rail node

Robinson Nuclear Power Plant

Savannah River Site

Site C

Site K

Site L

Summer Nuclear Power Plant

\section{Tennessee}

East Tennessee Technology Park (K-25 Site)

Oak Ridge National Laboratory

Sequoyah Nuclear Power Plant

Watts Bar Nuclear Power Plant

Y-12 Plant

\section{Texas}

Comanche Peak Nuclear Power Plant

Pantex Plant

South Texas Nuclear Power Plant

\section{Utah}

EnviroCare

Tooele Army Depot

\section{Vermont}

Vermont Yankee Nuclear Power Plant

\section{Virginia}

North Anna Nuclear Power Plant

Surry Nuclear Power Plant

Nearest rail node

\section{Washington}

Columbia Nuclear Power Plant

Hanford Site (Westinghouse Hanford, PNL)

\section{West Virginia}

Morgantown Energy Technology Center

\section{Wisconsin}

Kewaunee Nuclear Power Plant nearest rail node

La Crosse Nuclear Power Plant

Point Beach Nuclear Power Plant nearest rail node no direct rail access

SENECA

NS

ROBINSON NP

CSXT

SRS SITE C

USG

SRS SITE K

USG

SRS SITE L

USG

SUMMER NP

NS

EAST TN TECH PARK

$<\mathrm{C} 3>$

no direct rail access

SEQUOYAH NP

NS

WATTS BAR NP

NS

Y12 PLANT

CSXT

COMANCHE PEAK NP

FWWR

PANTEX PLANT

BNSF

SOUTH TEXAS

UP

CLIVE

UP

TOOELE ARMY DEPOT

UP

VERMONT YANKEE NP

NECR

NORTH ANNA NP

CSXT

no direct rail access

ZUNI

NS

COLUMBIA NP

USG

HANFORD SITE

USG

MORGANTOWN

CSXT

no direct rail access

DENMARK

$\mathrm{CN}$

LA CROSSE NP

BNSF

no direct rail access

DENMARK

\section{$\mathrm{CN}$}


Page intentionally left blank 


\section{INTERNAL DISTRIBUTION}

1. B. L. Bhaduri

2. P. E. Johnson

3. A. S. Loebl

4. S. B. Ludwig

5. R. D. Michelhaugh

\author{
6. R. R. Rawl \\ 7. B. A. Worley \\ 8. Central Research Library \\ 9. ORNL Laboratory Records-RC \\ 10-11. ORNL Laboratory Records-OSTI
}

\section{EXTERNAL DISTRIBUTION}

12. S. C. Hamp, U.S. Department of Energy, Albuquerque Operations Office, P.O. Box 5400, Albuquerque, NM 87185-5400 